\title{
The amazing potential of fungi: 50 ways we can exploit fungi industrially
}

Kevin D. Hyde ${ }^{1,2,3,4,5,9} \cdot$ Jianchu $\mathrm{Xu}^{1,10,21} \cdot$ Sylvie Rapior ${ }^{22} \cdot$ Rajesh Jeewon $^{18}$ - Saisamorn Lumyong ${ }^{9,13}$. Allen Grace T. Niego ${ }^{2,3,20}$ - Pranami D. Abeywickrama ${ }^{2,3,7}$ • Janith V. S. Aluthmuhandiram ${ }^{2,3,7}$. Rashika S. Brahamanage ${ }^{2,3,7} \cdot$ Siraprapa Brooks ${ }^{3}$ - Amornrat Chaiyasen ${ }^{28} \cdot$ K. W. Thilini Chethana ${ }^{2,3,7}$. Putarak Chomnunti ${ }^{2,3} \cdot$ Clara Chepkirui $^{12}$ - Boontiya Chuankid ${ }^{2,3} \cdot$ Nimali I. de Silva ${ }^{1,2,4,13}$. Mingkwan Doilom ${ }^{1,4,13}$. Craig Faulds $^{6}$. Eleni Gentekaki ${ }^{3}$ - Venkat Gopalan ${ }^{14}$ - Pattana Kakumyan ${ }^{2,3}$. Dulanjalee Harishchandra ${ }^{2,3,7}$. Hridya Hemachandran ${ }^{24}$. Sinang Hongsanan ${ }^{26,27}$ - Anuruddha Karunarathna ${ }^{2,17}$. Samantha C. Karunarathna ${ }^{1}$ - Sehroon Khan ${ }^{10}$ - Jaturong Kumla ${ }^{13,9} \cdot$ Ruvishika S. Jayawardena ${ }^{2,3}$. Jian-Kui Liu' ${ }^{11}$ - Ningguo Liü2,3 . Thatsanee Luangharn ${ }^{1,21,22,29}$ - Allan Patrick G. Macabeo ${ }^{12,23}$. Diana S. Marasinghe ${ }^{2,3} \cdot$ Dan Meeks $^{19} \cdot$ Peter E. Mortimer ${ }^{1,10} \cdot$ Peter Mueller $^{19} \cdot$ Sadia Nadir $^{10,15,21}$. Karaba N. Nataraja ${ }^{16}$ - Sureeporn Nontachaiyapoom ${ }^{3}$ - Meghan O'Brien ${ }^{19}$ - Watsana Penkhrue ${ }^{9,13}$. Chayanard Phukhamsakda ${ }^{2,3}$. Uma Shaanker Ramanan ${ }^{16,25}$. Achala R. Rathnayaka ${ }^{2,3}$ - Resurreccion B. Sadaba ${ }^{29}$. Birthe Sandargo $^{12}$ - Binu C. Samarakoon ${ }^{2,3}$ - Danushka S. Tennakoon ${ }^{2,3} \cdot$ Ramamoorthy Siva $^{24}$. Wasan Sriprom $^{9,13}$. T. S. Suryanarayanan ${ }^{30}$ - Kanaporn Sujarit ${ }^{9,13} \cdot$ Nakarin Suwannarach $^{9,13}$. Thitipone Suwunwong ${ }^{3,8} \cdot$ Benjarong Thongbai $^{12} \cdot$ Naritsada Thongklang $^{2}$ - Deping Wei $^{1,2,3,17}$.

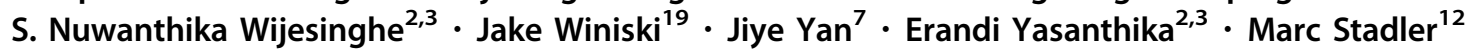

Received: 5 April 2019/ Accepted: 20 May 2019/ Published online: 3 July 2019

(C) The Author(s) 2019

\begin{abstract}
Fungi are an understudied, biotechnologically valuable group of organisms. Due to the immense range of habitats that fungi inhabit, and the consequent need to compete against a diverse array of other fungi, bacteria, and animals, fungi have developed numerous survival mechanisms. The unique attributes of fungi thus herald great promise for their application in biotechnology and industry. Moreover, fungi can be grown with relative ease, making production at scale viable. The search for fungal biodiversity, and the construction of a living fungi collection, both have incredible economic potential in locating organisms with novel industrial uses that will lead to novel products. This manuscript reviews fifty ways in which fungi can potentially be utilized as biotechnology. We provide notes and examples for each potential exploitation and give examples from our own work and the work of other notable researchers. We also provide a flow chart that can be used to convince funding bodies of the importance of fungi for biotechnological research and as potential products. Fungi have provided the world with penicillin, lovastatin, and other globally significant medicines, and they remain an untapped resource with enormous industrial potential.
\end{abstract}

Keywords Biocontrol $\cdot$ Biodiversity $\cdot$ Biotechnology $\cdot$ Food $\cdot$ Fungi $\cdot$ Mushrooms

\section{Table of contents}

From basic to applied research, prototypes and products contribution by Birthe Sandargo, Marc Stadler

Jianchu Xu

j.c.xu@cgiar.org

Extended author information available on the last page of the article
Strategies against human disease

1. Antibacterial antibiotics contribution by Clara Chepkirui, Benjarong Thongbai, Marc Stadler,

2. Antimycotics contribution by Benjarong Thongbai, Marc Stadler

3. Biofilm inhibitors contribution by Benjarong Thongbai, Marc Stadler 
4. Anti-cancer agents contribution by Chayanard Phukhamsakda, Marc Stadler

5. Anti-diabetes contribution by Achala R. Rathnayaka, Marc Stadler

6. Improving nerve functioning contribution by Benjarong Thongbai, Marc Stadler

7. Fungi in Traditional Chinese Medicine contribution by Thatsanee Luangharn, Marc Stadler

8. Cardiovascular disease control by fungi contribution by Anuruddha Karunarathna, Marc Stadler

9. Antiviral agents contribution by Allan Patrick G. Macabeo, Marc Stadler

10. Immunosuppressive and immunomodulatory agents from fungi contribution by Clara Chepkirui, Marc Stadler

\section{Strategies against plant disease}

11. Biocontrol of plant disease using endophytes contribution by Nimali I. de Silva, Siraprapa Brooks

12. Biocontrol of insects using fungi contribution by Allen Grace T. Niego

13. Biocontrol of nematodes and fungal nematizides contribution by Diana S. Marasinghe, Clara Chepkirui

14. Biocontrol of weeds and herbicides from fungi contribution by Pranami D. Abeywickrama, Jiye Yan

15. Fungal antagonists used in post-harvest disease control contribution by Binu C. Samarakoon

16. Bio control of rusts and smuts by antagonistic fungi contribution by Rashika S. Brahmanage

\section{Enhancing crops and forestry}

17. Biofertilizers contribution by Mingkwan Doilom

18. Arbuscular mycorrhizae as biofertilizers contribution by Amornrat Chaiyasen, Saisamorn Lumyong

19. Application of ectomycorrhizal fungi in forestry contribution by Jaturong Kumla, Saisamorn Lumyong

20. Use of orchid mycorrhizae and endophytes in biotechnology

contribution by Nimali I. de Silva, Sureeporn Nontachaiyapoom

21. Growth promoting hormones from fungi contribution by Saisamorn Lumyong

22. Mitigating abiotic stress in plants: the endophyte method contribution by Karaba N. Nataraja, Uma Shaanker Ramanan

\section{Food and beverages from fungi}

23. Growing mushrooms in compost contribution by Naritsada Thongklang

24. Growing mushrooms in bags contribution by Samantha Karunarathna

25. Growing mushrooms in the field contribution by Peter E. Mortimer, Samantha C. Karunarathna

26. Modern mushroom production: an automated factory process contribution by Jianchu $\mathrm{Xu}$

27. New edible mushrooms contribution by Samantha Karunarathna

28. Agaricus subrufescens contribution by Naritsada Thongklang

29. Using fungi to enhance food value contribution by Danushka S. Tennakoon

30. Food colouring from filamentous fungi contribution by Wasan Sriprom and Saisamorn Lumyong

31. Food flavouring contribution by S. Nuwanthika Wijesinghe

32. What is mushroom stock? Products, process and flavours contribution by Deping Wei

33. Fungi in making tea contribution by Ningguo Liu, Jack JK Lui

34. Wine, beer and spirits contribution by Sinang Hongsanan

35. Functional foods and nutraceuticals contribution by Boontiya Chuankid

36. Harvesting the untapped probiotic potential of fungi contribution by Eleni Gentekaki, Achala R. Rathnayaka

\section{Saving the planet}

37. Agricultural waste disposal contribution by Putarak Chomnunti, Craig Faulds

38. Mycoremediation: Fungi to the rescue contribution by Dulanjalee Harishchandra, Jiye Yan

39. Mycofumigation using Muscodor contribution by Nakarin Suwannarach, Saisamorn Lumyong

40. Biomass to biofuel: unmasking the potential of lesser-known fungi

contribution by Venkat Gopalan, T.S. Suryanarayanan

41. Packed-bed bioreactor for mycomaterial production contribution by Peter Mueller, Dan Meeks, Meghan O'Brien, Jake Winiski 
42. Fungal degradation of plastics: A hidden treasure for green environment contribution by Sehroon Khan, Sadia Nadir

43. Polycyclic aromatic hydrocarbon degradation by basidiomycetes

contribution by Allen Grace T. Niego, Resurreccion B. Sadaba

44. Can fungi help modify the sustainable soil enhancer biochar?

contribution by Thitipone Suwunwong, Craig Faulds

\section{Commodities}

45. Fungi and cosmetics contribution by Erandi Yasanthika

46. Agarwood contribution by S. Nuwanthika Wijesinghe

47. Fungal enzymes contribution by Pattana Kakumyan

48. Preservatives contribution by Benjarong Thongbai

49. Organic acids contribution by Janith V.S. Aluthmuhandiram

50. Textile dyes contribution by Ruvishika S. Jayawardena

\section{The future}

Functional genomics and the search for novel antiinfectives

contribution by K.W. Thilini Chethana, Jiye Yan and Birthe Sandargo

\section{From basic to applied research, prototypes and products}

Fungi have both good and bad facets (Pointing and Hyde 2001). They are essential for nutrient cycling because of their ability to degrade cellulose and lignin (Pointing et al. 2001). On the other hand, they cause serious human, animal and plant diseases and have numerous negative aspects on human life (Hyde et al. 2018a). Fungi are, however, also relatively understudied, but are an essential, fascinating and biotechnologically useful group of organisms with an incredible biotechnological potential for industrial exploitation. In this paper, we detail 50 ways in which we can potentially exploit fungi. We provide notes and examples for all potential exploitations and give examples from our own work and the work of others. We also provide a flow chart that can be used to convince funding bodies just how important fungi are and their potential for biotechnological research and potential products.
While several of our chapters are dealing with marketed products that even include blockbuster pharmaceuticals, such as the beta-lactam antibiotics, the statins and cyclosporine, others are dedicated to newly upcoming areas that still remain to be explored. Other chapters treat relatively small market segments that may expand in the future. For example, the consumers around the world now increasingly prefer natural compounds over synthetic chemicals and even in the industrial sectors that produce commodity chemicals, there is now an increased interest in development of sustainable biotechnological processes, in order to obtain new natural products that can eventually replace traditional synthetics. As compared to other biological sources, in particular plants, fungi have the great advantage that they can be grown in large bioreactors at an industrial scale, and suitable processes for their cost-efficient fermentation have been available for many decades, e.g. for production of certain organic acids, enzymes and antibiotics. As exemplified by the recent studies of the Thai mycobiota, modern polyphasic taxonomic approaches are constantly revealing a plethora of new and undescribed species even in the fairly well-known genera of fungi like Agaricus (Hyde et al. 2018b). Even the majority of the known species in the fungal kingdom are virtually untapped with regard to potential applications, also because they were never cultured and studied for their growth characteristics and physiology. New methods and protocols have to be developed for this purpose, and this implies that substantial basic research must be carried out before the exploitation of the novel organisms can be envisaged.

Although fungi have so many potential uses, research on their potential applications is in general poorly funded and much of the research that is being carried out in academia is fundamental, even in areas that belong to the fields of biotechnology and applied mycology. For example, screening fungi for production of antibiotics by antagonistic culture testing has often been reported, but is unlikely to lead to industrial projects. Often, it will take over a decade even to bring a given project based on a novel fungal metabolite into the preclinics, and even this is only possible by joint, interdisciplinary efforts of biologists, biotechnologists, pharmacists and chemists. Moreover, the Big Pharma industry has recently downsized their capacities for in-house research, meaning that the academic sector (sometimes supported by smaller companies or organisations like the Bill and Melinda Gates Foundation and the Wellcome Trust) has become more and more involved in the preclinical evaluation of new compounds.

Investing in basic research may seem, at first sight, a costly affair. However, there are numerous examples of the 
past demonstrating why investing in basic research pays off in the long run, and even more reasons, why it is today more important than ever to renew an interest in basic research on fungi. But how to convince funders, in particular from the private sector, to invest into researchers doing basic research on fungi?

There are, no doubt, areas of research, which are of utmost importance to the entire world, yet are considered valueless to the pharmaceutical industry. One of these is the search for novel anti-infectives, as the world is running out of antibiotics (Hesterkamp 2017; WHO report 2017). It has long been seen as a tedious process to obtain novel antibiotics from living organisms.

However, the focus in the past has been on the same bacterial and fungal genera, such as Streptomyces in the Actinobacteria and common soil moulds like Aspergillus and Penicillium in the filamentous fungi (Karwehl and Stadler 2017). Since almost no novel carbon skeletons have been discovered from these common soil microbes in the past 20 years, it makes much more sense to study the numerous species that are constantly being discovered and shown to belong to new phylogenetic groups.

In our review, we present fungi, in particular Basidiomycota, as a still underexplored, highly promising source of anti-infectives, immunosuppressants, and other pharmaceuticals (see Badalyan et al. 2019; Sandargo et al. 2019a) that is nowhere near dried up. We give examples on recent developments of turning fungal natural products into commercial drugs and give an overview of the current state of applied research in this field.

In the past, fungal natural products have also led to some blockbusters and various developmental candidate compounds for the agrochemical industry (Bills and Gloer 2016). However, the uncontrolled usage of such fungal pesticides has led to the development of more and more resistances against these agrochemicals (Lucas et al. 2015). A more controlled approach of crop protection is therefore advisable. More basic research is needed to understand natural processes, and thereby allow for the search of natural control agents. In the entries dealing with "Strategies against plant disease", we show the great potential of fungi as biocontrol agents. We give examples of how fungal biocontrol agents can help save the Agro sector tremendous amounts of money, if companies are given the opportunity to produce cost efficient biocontrol agents. In a likewise manner, the part on "Enhancing crops and forestry" deals with the current research on ectomycorrhiza and their potential application as natural biofertilizers.

With the new trend to a more sustainable, health-oriented living, and constant reports of hazardous chemicals found in food and cosmetics, the demand for more ecological, more "natural" alternatives is high. This is again, where fungi can step in. In the entries on "Food and beverages from fungi" and "Commodities", we present examples of how basic research on fungi has made its way into the food and beverage, but also the textile and flavour industry. Finally, in the part on "Saving the planet" we illustrate the great potential of fungi towards a more sustainable living and how fungi can assist to cope with some potential future challenges that are threatening human civilisation. A diagram illustrating all potential beneficial uses of fungi that are treated herein is given in Fig 1.

\section{Strategies against human disease}

The scientific community recently celebrated the $90^{\text {th }}$ anniversary of Sir Alexander Fleming's discovery of penicillin, which marked the starting point of the era of antibiotic chemotherapy. As outlined by Karwehl and Stadler (2017), among the numerous antibiotics that were discovered over the next 50 years, relatively few compound classes were derived from fungi. The latter include the cephalosporins (Newton and Abraham 1955), which belong to the same class as the penicillins, i.e. the beta glucan antibiotics, as well as fusidic acid (Godtfredsen et al. 1962) and pleuromutilin (Novak and Shlaes 2010; Sandargo et al. 2019a). Their chemical structures $(\mathbf{1 - 4})^{1}$ are depicted in Fig. 2. Most other commercial antibiotics are actually derived from Streptomyces species and other actinobacteria, or even from other prokaryotes. For details of the history of research on antibiotics, we refer to the review by Mohr et al. (2017), as this does not fall within the scope of the current paper. As we cannot cover the entire field in this paper, we will give a brief overview on antibacterials, antimycotics and biofilm inhibitors and illustrate their usages with some examples of marketed drugs as well as other compounds that have recently been discovered.

\section{Antibacterial antibiotics}

The term "antibiotics" is used in the literature with different definitions. The industry mainly use it for antibacterial agents, but the definition that we prefer here, which was adapted from the original one coined by Waksman (1947), i.e., an antibiotic is "a chemical substance, produced by micro-organisms (including fungi), which has the capacity to inhibit the growth of and even to destroy bacteria and other micro-organisms". The natural functions of antibiotics can easily be explained, resulting from the high competition between fungi, bacteria and other organisms in

\footnotetext{
1 All chemical structures of fungal secondary metabolites presented in this paper have been numered consecutively in bold typeset in both, the figures and the corresponding text.
} 


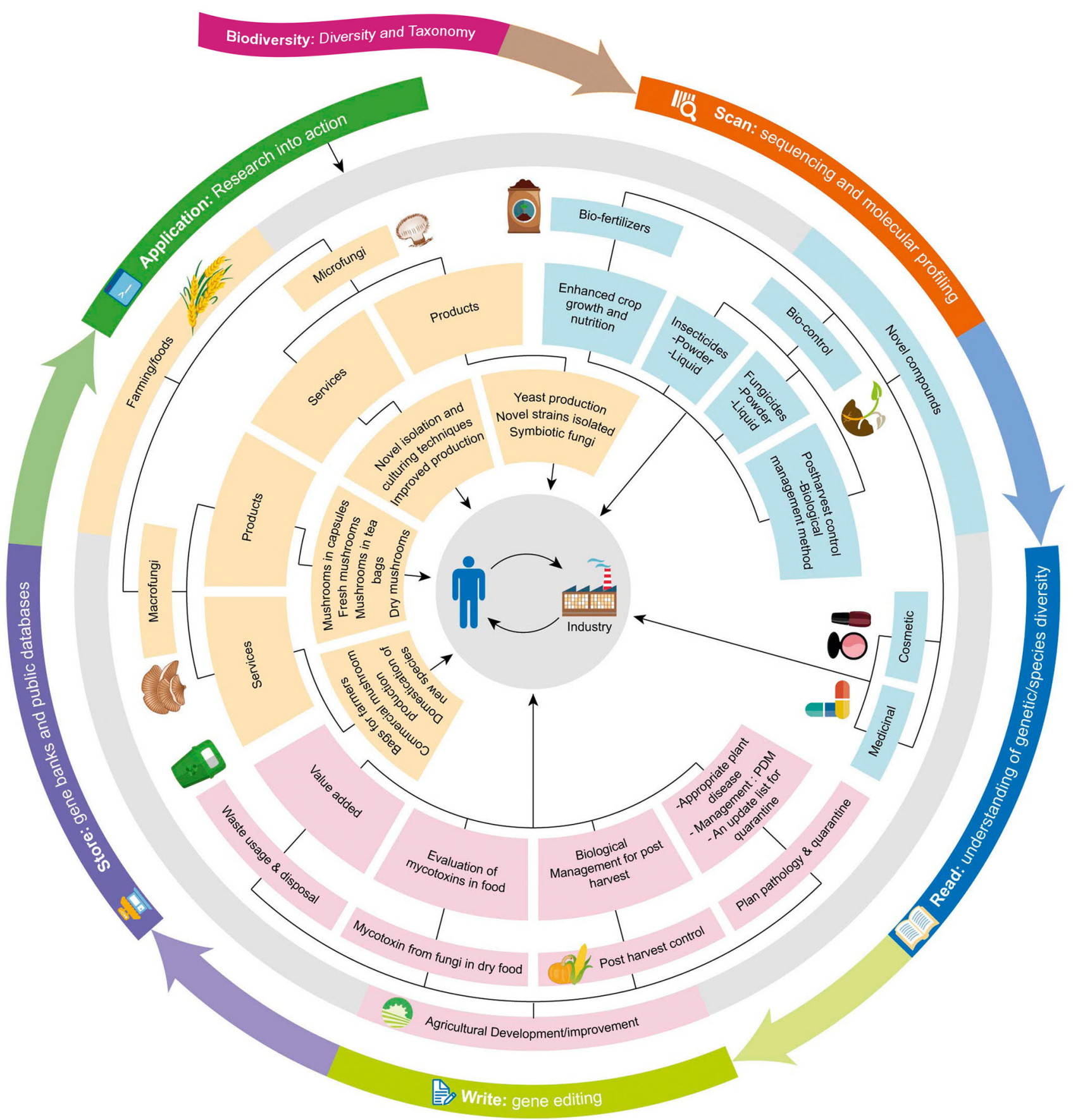

Fig. 1 Diagram showing the potential use of fungi in biotechnology. The cycle starts with basic biodiversity research, which in turn leads to cultures placed in the central culture collection. The cultures are

substrates such as soil, dung and plant debris. If a given organism has acquired the ability to produce a certain secondary metabolite by which it can kill the competing organisms that dwell in the same habitat, it is considered to possess a selective advantage that ultimately increases its fitness (Shearer 1995). Therefore it should come as no surprise that one large experimental study concluded that the majority of filamentous fungi are able to produce then used for applied research, which in turn leads to products in the form of the items discussed in the entries of this paper

antibiotic compounds (Bills et al. 2009). Bills and Gloer (2016) summarized numerous important facts concerning the current state of the art in research on fungal secondary metabolites and concentrated heavily on the biochemical and genetic background of their biosynthesis.

We are currently living in the "post-antibiotic" era, where both, the numbers and percentages of multi-resistant bacterial and fungal pathogens against the established 
Fig. 2 Chemical structures of fungal metabolites that were developed to antibacterial drugs
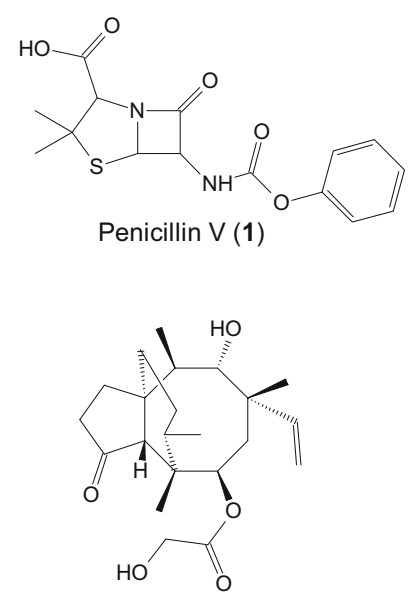

Pleuromutilin (4)<smiles>CC(=O)OCC1=C(C(=O)O)N2C(=O)C(NC(=O)CCCC(N)C(=O)O)C2SC1</smiles>

Cephalosporin C (2)

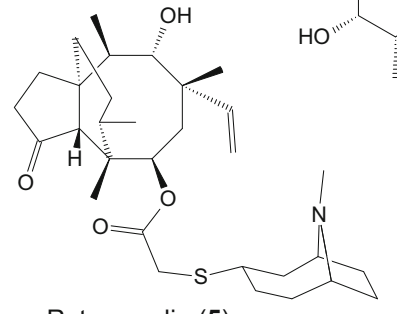

Retapamulin (5) antibiotics are drastically increasing, while the number of new therapeutic agents and developmental candidates has decreased (Cooper and Shlaes 2011). The reasons for this development are manifold, but the phenomenon is primarily due to the fact that the majority of pharmaceutical companies have lost interest in Research and Development on natural products and/or given up their activities in the anti-infectives sector. Experts around the world are now giving warnings about the serious consequences that the lack of antibiotics-in particular against the multi-resistant Gram negative human pathogenic bacteria-can have (Friedman et al. 2016). After two decades of neglect, efforts of both the private and the academic sector on the discovery of new antibiotics have substantially increased.

The pipeline for antibacterial antibiotics (Hesterkamp 2017) shows that there are still some compounds under development, but the majority of those have been optimised from old compounds with known modes of action, e. g. by chemical modifications. Therefore, it is likely that the resistant pathogens will easily find a way to cope with the new products, once they have reached the market. The aforementioned mutilins, which are derived from fermentation of the basidiomycete Clitopilus passeckerianus and subsequent semisynthesis, therefore represent the "newest" compound class that has been registered as an antibacterial drug. A derivative, retapamulin (5), was launched for use as a topical antibiotic against skin infections, and several further derivatives are undergoing clinical trials as systemic antibiotics. In general, basidiomycete cultures are much more difficult to handle with respect to large scale production of secondary metabolites, since they grow rather slowly and often have low yields. For the production of pleuromutilin, however, Bailey et al. (2016) managed to increase the yields substantially after the transfer of the biosynthetic genes into a fast growing heterologous
Aspergillus host, which can more easily be handled in the production process. This accomplishment can give rise to some hope that in the future, more of the hitherto neglected, unique biologically active metabolites of basidiomycetes can be made accessible to preclinical development.

\section{Antimycotics and fungicides}

Whereas multi-resistant bacterial pathogens are very high on the agenda of both the press and funding agencies, relatively little attention is presently being paid to the fact that the number of resistant pathogenic fungi is also on the rise. This topic was treated by Hyde et al. (2018a), we refer to it for the most important ant threatening human pathogenic fungal organisms. In fact, there are only a handful of efficient compound classes on the market that are used in antimycotic chemotherapy, including griseofulvin (6), which was already discovered by Grove et al. (1952; Fig. 3). The newest class of antimycotics that were launched to the market are the echinocandins (e.g., pneumocandin $B_{0}$ (7) (Denning 2002). The biosynthesis of these highly complex lipopeptides relies on PKS-NRPS hybrid gene clusters (Chen et al. 2013). They are being produced biotechnologically by large scale fermentation using different fungi that are not phylogenetically related and subsequent semisynthesis. The knowledge about the molecular mechanisms of their biosynthesis may in the future lead to the concise manipulation of the production process that can be directed towards new natural derivatives. Interestingly, a comparative genomics study by Yue et al. (2015) has revealed rather high homologies among the biosynthesis gene clusters of the producer organisms that belong to three different classes of Ascomycota, namely Dothideomycetes, Eurotiomycetes, and Leotiomycetes. Possibly, this has 
Fig. 3 Chemical structures of fungal metabolites with antifungal activity against human pathogens

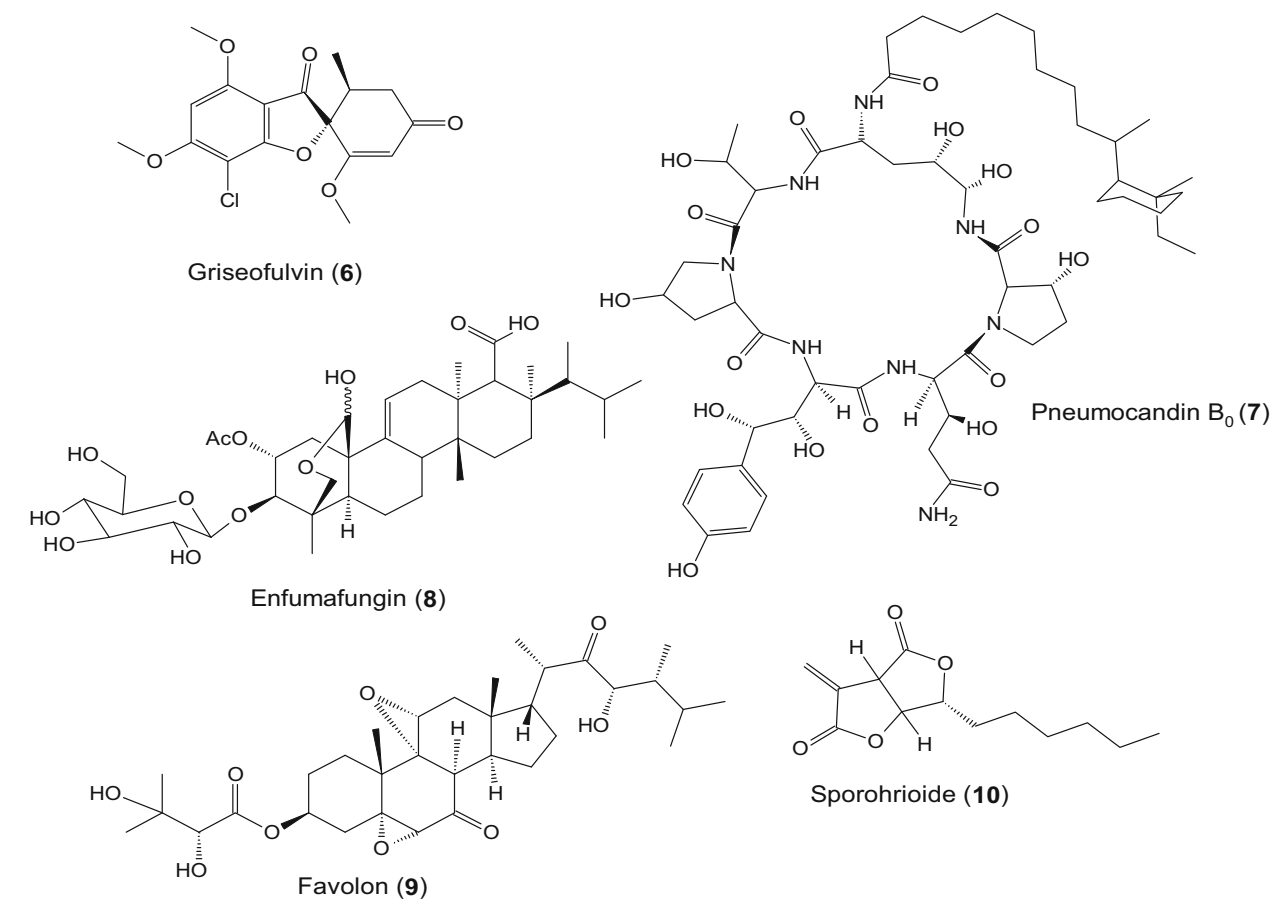

been due to horizontal gene transfer during the evolutionary history of these organisms.

Recent efforts aimed at the discovery of novel antifungal agents have resulted in a number of developmental projects, such as enfumafungin (8) from Hormonema spp. (Peláez et al. 2000). This compound class may soon yield the first pharmaceutical drug for use in humans that originated from a fungal endophyte, over 15 years after their first discovery. Even the biosynthesis genes encoding for these unique triterpenoids (Fig. 3) has only recently been identified (Kuhnert et al. 2018).

The search for novel antimycotics and fungicides has also resulted in the rediscovery of "old" compounds that may become more interesting in the future because they have originally been found in a screening for agrochemical fungicides and were never evaluated for their effects on human fungal pathogens or their mode of action. While the strobilurins, which are a very commercially successful antifungal agents in agriculture (Sauter et al. 1999), have been found inefficient or too toxic for application in humans, many other metabolites with pronounced antifungal effects were apparently never tested on their efficacy against human pathogens. A recent example for such rediscoveries is favolon (9), which is actually a cometabolite of strobilurins produced by the invasive basidiomycete Favolaschia calocera (Chepkirui et al. 2016) and was originally isolated by Anke et al. (1995). Like the sporothriolodes (10) from the xylarialean fungus Hypoxylon monticulosum (Surup et al. 2014; Fig. 3; now classified in the new genus Hypomontagnella as H. monticulosa; cf.
Lambert et al. 2019), this metabolite shows very strong antifungal effects that are not accompanied by prominent cytotoxicity.

\section{Biofilm inhibitors}

Scientists are exploring different avenues to combat infectious diseases caused by both bacterial and fungal pathogens, for which the inhibition of biofilm formation is one of the most promising leads. Abraham and Estrela (2016) reported that fungal metabolites are becoming increasingly explored for their potential to inhibit the formation of biofilms, e.g. by interfering with quorum sensing, and some compounds have already been discovered that can even destroy pre-formed biofilms. A recent example is coprinuslactone (11) (de Carvalho et al. 2016; Fig 3), a small molecule derived from the edible mushroom Coprinus comatus, which acts against Pseudomonas aeruginosa biofilms. Other examples include roussoellenic acid (12) from a Roussoella sp. (Phukhamsakda et al. 2018), which is active against biofilm formation in Staphylococcus aureus, as well as microporenic acid A (13) from a Kenyan basidiomycete (Chepkirui et al. 2018; Fig. 4), which can not only inhibit biofilm formation in both Staphlococcus aureus and the human pathogenic yeast, Candida albicans, but even destroys pre-formed biofilm in C. albicans at rather low concentrations. These compounds do not have prominent antimicrobial activities and therefore their application is unlikely to raise resistance. The biofilm inhibitors are very promising candidates for use in 


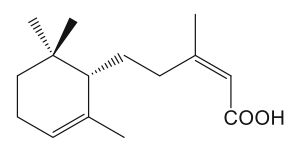

Roussoellenic acid (11)

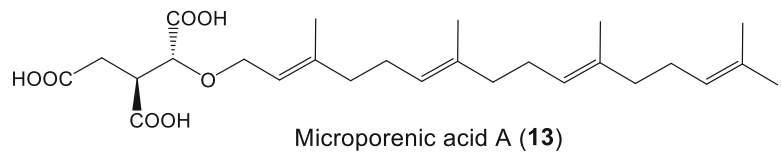

Fig. 4 Chemical structures of fungal metabolites with biofilm inhibition activities

combination therapy with antibiotics. In several studies, biofilm inhibitors were shown to enhance the activity of the antibiotics by increasing their ability to penetrate the biofilms.

These examples illustrate that fungi are under-explored with respect to novel antibiotics and other therapeutic agents, and that it is certainly worthwhile to expend more effort in this area of research with an emphasis on hitherto neglected species from regions and habitats that have not yet been studied systematically. Fungi have much to offer in terms of novel chemistry: due to the advent of revolutionary techniques in genomics, transcriptomics, bioinformatics, analytical chemistry and biotechnological process development, we can now explore the chemical diversity of the mycobiota much more concisely than ever before. Evidence is also accumulating that novel phylogenetic lineages or hitherto neglected taxonomic and ecological groups of fungi can now much more easily be recognized and subjected to the exploitation of their secondary metabolome. However, more public funding is needed to assure that the substantial know-how that has been acquired over many decades does not become forgotten, and that the next generation of researchers will also still be able to work on novel, hitherto unexplored fungal groups, rather than only on model organisms.

\section{Anti-cancer agents}

Cancer is the second leading cause of mortality after cardiovascular disease, with an estimated 9.6 million cancerrelated deaths in 2018 (GBD 2015). Cancer is a multifactorial disease characterized by the loss of growth factors that control the proliferation and division of cells. These abnormal malignant cells can evade the tumour suppressor factors of the human immune system, then develop to tumours and destroy adjacent tissues (Saeidnia and Abdollahi 2014). There are several treatments for cancer, administered according to developmental state of the disease. Chemotherapy, radiation therapy, surgery and immunotherapy are all important elements of cancer treatment. However, while many cytotoxic agents are known to Science (which could in principle serve as chemotherapeutic agents), only few of them specifically target tumour cells and are less toxic to regular, healthy human tissue (Petrelli et al. 2012; Cai et al. 2013; Zugazagoitia et al. 2016). Targeted therapy, usually the conjugated element for cancer treatments, delivers drugs to genes or proteins that are specific to cancer cells or the environmental tissues that promotes the growth of cancer (Padma 2015). Fungi are an importance source for natural product discovery, albeit most anticancer drugs are retrieved from plants and bacteria. In this entry, we describe several promising natural products derived from fungi and highlight some of the chief compounds that are currently in the clinical and preclinical developmental stage (Fig. 5).

Irofulven (14) is a semi-synthetic derivative of illudin S (15), a natural toxin isolated from Omphalotus illudens (Jack O'Lantern mushroom; cf. Chin et al. 2006; Movassaghi et al. 2006). Irofulven interferes with DNA replication-complexes and cell division in DNA synthesis. The abnormal cells in S-phase lead to apoptotic cell death (Walser and Heinstein 1973; Jaspers et al. 2002). The antitumour activities of irofulven have been evaluated in phase I and II clinical trials with promising results against a variety of cancers, including those in the brain and central nervous system, breast, blood, colon, sarcoma, prostate, lungs, ovarian and pancreas (Alexandre et al. 2004; Miyamoto et al. 2018; Topka et al. 2018). Sandargo et al. (2019a) have recently described the state of the art, including some exciting new illudin conjugates that show superior in vitro activities than irufulven and are presently under early preclinical development.

Aphidicolin (16) is a tetracyclic diterpene with antiviral and antimitotic properties. The compound was originally isolated from "Cephalosporium aphidicola" (currently valid name: Akanthomyces muscarius) and later also reported from Nigrospora sphaerica (Bucknall et al. 1973; Starratta and Loschiavo 1974). Aphidicolin competes for the specific binding site on DNA polymerase $\alpha, \delta$, and $\varepsilon$ enzymes. Its mechanism of action and efficacy have been intensively tested in clinical trials (Crosetto et al. 2013; Ayob et al. 2017), but so far it has not become a marketed drug.

Other anticancer lead compounds derived from fungi include leptosins F (17) and C (18) isolated from Leptoshaeria $\mathrm{sp}$., which showed antitumor activity in mouse embryos (Yanagihara et al. 2005; Pejin et al. 2013); $\beta$ glucans, the polysaccharides that are naturally found on the cell walls of fungi (Chan et al. 2009; Bashir and Choi 2017); as well as palmarumycin (Powis et al. 2006) and spiropreussione A (Chen et al. 2009). The latter 
Fig. 5 Chemical structures of promising natural products derived from fungi with anticancer activity

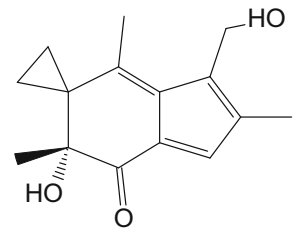

Irofulven (14)

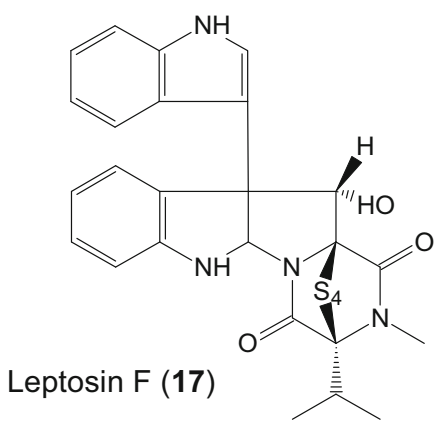

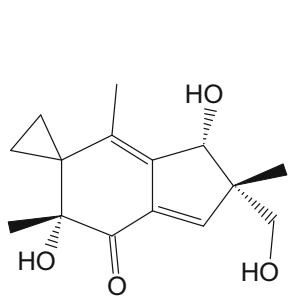

Illudin S (15)

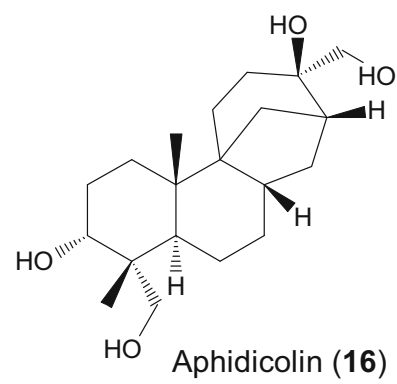

(16)

Leptosin C (18)

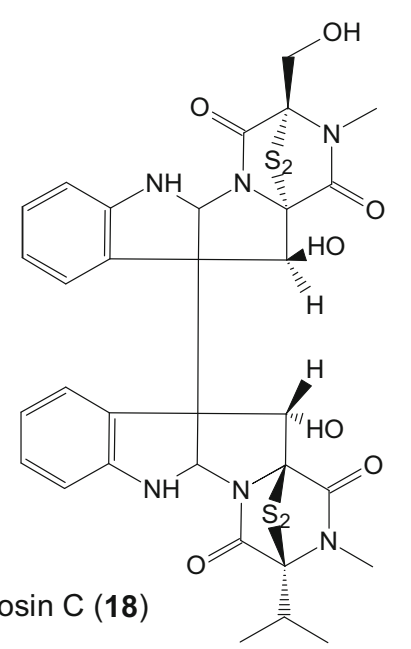

compounds, however, have only demonstrated these activities in vitro, and it is not clear whether they will eventually reach the late exploratory stage of preclinical development.

\section{Anti-diabetes}

Diabetes mellitus, also known simply as diabetes, is a chronic metabolic disorder (De Silva et al. 2012). People who suffer from diabetes cannot produce or effectively use insulin in the body. Due to this insulin imbalance, they have high amounts of glucose in their blood. There are two common types of diabetes, i.e. type 1 diabetes (insulin dependent diabetes mellitus) and type 2 diabetes (noninsulin-dependent diabetes mellitus).

Patients with type 1 diabetes cannot produce insulin, due to the lack of functions of the insulin-secreting beta cells in the pancreas (Meier et al. 2005). They must take insulin continuously every day to stay alive. Type 1 diabetes mostly affects children and adolescent patients, and it represents $5-10 \%$ of total diabetes cases worldwide. Patients with type 2 diabetes cannot produce sufficient insulin or cannot effectively metabolize it. This form of the disorder commonly affects elderly people and accounts for 90-95\% of all diabetes cases (Hameed et al. 2015). People worldwide suffer from diabetes mellitus and $7 \%$ of the world's adult population is affected by the disease (Philippe and Raccah 2009). In 2017, the largest number of diabetic patients, around 114 million, was recorded in China. Roughly 73 million diabetic patients were recorded in India, and 30 million were recorded in the United States. (https://www.statista.com/statistics/281082/countries-withhighest-number-ofdiabetics/).

There are many negative consequences for patients if diabetes remains untreated, such as blindness, kidney failure, depression, cardiovascular diseases, cancer and even death (Gerstein et al. 2011; Hansen et al. 2012; Huang et al. 2018). Retinopathy (damage of the retina, leading to blindness) and neuropathy (damage of the nervous system) are some of the most severe complications that have been attributed to diabetes (De Silva et al. 2012; Sobngwi et al. 2012).

Many Basidiomycota, such as Agaricus bisporus, Cyclocybe aegerita, C. cylindracea and Tremella fuciformis are used as medicine for the treatment or prophylaxis of type 2 diabetes. These mushrooms help patients avoid high levels of glucose because they contain the least amount of digestible carbohydrates in the diet (Poucheret et al. 2006). Bioactive metabolites, which are isolated from medical mushrooms and their cultured mycelia, act as biological antihyperglycemic agents in diabetes treatment (Table 1) (De Silva et al. 2012). Extracts of Inocutis levis (Hymenochaetaceae) have been reported to possess utility as a remedy for diabetes because they increase insulin resistance, insulin sensitivity and glucose uptake in tissues and hence help to control blood glucose levels (Ehsanifard 
Table 1 Anti-diabetic effects of some medical mushroom species

\begin{tabular}{|c|c|c|c|c|}
\hline Mushroom species & Common name & Bioactive compounds & $\begin{array}{l}\text { Experimental setup and } \\
\text { observed effects }\end{array}$ & References \\
\hline Agaricus bisporus & $\begin{array}{l}\text { White button } \\
\text { mushroom }\end{array}$ & Dry fruiting body extract & $\begin{array}{l}\text { Streptozotocin-injected diabetic } \\
\text { Sprague Dawley } \\
\text { rats (reduced blood glucose } \\
\text { level) }\end{array}$ & $\begin{array}{l}\text { Jeong et al. } \\
(2010) \\
\text { Yamac et al. } \\
(2010)\end{array}$ \\
\hline Agaricus campestris & $\begin{array}{l}\text { Field } \\
\text { mushroom }\end{array}$ & Aqueous fruiting body extract & & $\begin{array}{l}\text { Gray and Flatt } \\
\text { (1998) }\end{array}$ \\
\hline Agaricus subrufescens & $\begin{array}{l}\text { Almond } \\
\text { mushroom }\end{array}$ & $\beta$-Glucans from dried fruiting bodies & & $\begin{array}{l}\text { Kim et al. } \\
\text { (2005) }\end{array}$ \\
\hline Inonotus obliquus & $\begin{array}{l}\text { Chaga } \\
\text { mushroom }\end{array}$ & $\begin{array}{l}\text { Ethyl acetate fraction from dried } \\
\text { fruiting bodies }\end{array}$ & $\begin{array}{l}\text { Alloxan-induced diabetic mice } \\
\text { (reduced blood } \\
\text { glucose level) }\end{array}$ & $\begin{array}{l}\text { Lu et al. } \\
(2010)\end{array}$ \\
\hline $\begin{array}{l}\text { Ophiocordyceps sinensis } \\
\text { ("Cordyceps" sinensis) }\end{array}$ & $\begin{array}{l}\text { Caterpillar } \\
\text { fungus }\end{array}$ & $\begin{array}{l}\text { Polysaccharide from Ophiocordyceps } \\
\text { (Cordyceps) mycelia }\end{array}$ & $\begin{array}{l}\text { Streptozotocin- and alloxan- } \\
\text { induced diabetic } \\
\text { mice (reduced blood glucose } \\
\text { level/increased } \\
\text { the blood insulin level)1 }\end{array}$ & $\begin{array}{l}\text { Li et al. } \\
(2003) \\
\text { Li et al. } \\
(2006)\end{array}$ \\
\hline Hericium erinaceus & $\begin{array}{c}\text { Lion's mane } \\
\text { mushroom }\end{array}$ & $\begin{array}{l}\text { Methanol extract of the dried fruiting } \\
\text { body }\end{array}$ & $\begin{array}{l}\text { Streptozotocin-induced Wistar } \\
\text { rats (reduced } \\
\text { blood glucose level) }\end{array}$ & $\begin{array}{l}\text { Wang et al. } \\
(2005 \mathrm{~b})\end{array}$ \\
\hline Tremella mesenterica & $\begin{array}{c}\text { Yellow brain } \\
\text { mushroom }\end{array}$ & Fruiting body extract & $\begin{array}{l}\text { Streptozotocin-induced Wistar } \\
\text { rats (increased } \\
\text { insulin secretion and glucose } \\
\text { metabolism) }\end{array}$ & $\begin{array}{l}\text { Lo et al. } \\
\text { (2006) }\end{array}$ \\
\hline
\end{tabular}

et al. 2017). The fruiting bodies of Antrodia cinnamomea can be used to produce healthy foods and drugs that have anti-diabetes properties (Huang et al. 2018). Grifola frondosa has been used as medicine for type 2 diabetes, and its extracts can effect both hyperglycemia (when a high amount of glucose circulates in the blood) and hyperinsulinemia (when a high level of insulin circulates in the blood) (Poucheret et al. 2006).

Fungal products are sold as remedies for diabetes. Ophiocordyceps sinensis capsules, SX-Fraction, ReishiMax capsules and Tremella are some of the examples of anti-diabetic products made with medicinal mushrooms $(\mathrm{Li}$ et al. 2004; De Silva et al. 2012) and is claimed to decrease fasting blood glucose levels in type 2 diabetes. This medicine can also be used to reduce blood pressure and body weight. SX-Fraction is considered a major alternative for enhancing insulin sensitivity (Preuss et al. 2007). Tremella is a medicinal product used in Chinese medicine; produced from Tremella fuciformis (silver ear mushroom/ white jelly leaf mushroom), it is mainly used for reducing blood glucose and cholesterol levels (Li et al. 2004).

Future investigation is needed to clarify the long-term effects of taking medicinal mushroom products with other drugs. It is necessary to justify the use of medicinal mushroom products as anti-diabetes (De Silva et al. 2012).

\section{Improving nerve functioning}

Human neurodegenerative diseases, such as Alzheimer's, Huntington's, and Parkinson's disease, are disrupting neuronal populations in adults worldwide. The discovery and development of neuroactive compounds from medicinal mushrooms with the potential to improve nerve functioning has been extensively studied. Mushrooms, such as Antrodia camphorata, Ganoderma spp., Hericium erinaceus, Lignosus rhinocerotis and Pleurotus giganteus, have a long history of use in enhancing the peripheral nervous system. Nerve growth factors are important for the survival, maintenance, and regeneration of specific neuronal populations in the adult brain. It has been demonstrated that neurodegenerative diseases mostly occur because of the disappearance of nerve growth factors. Therefore, scientists have been attempting for over 20 years to discover fungi-derived neuroactive components which are able to cross the blood-brain barrier and induce the production of nerve growth factors.

Most of the potential neuroactive compounds, which may aid in the prevention or therapy of neurodegenerative diseases, have been discovered in Hericium erinaceus. This medical mushroom is known to produce two unique terpenoid classes, namely hericenones and erinacines, from its fruiting body and mycelia, which can stimulate synthesis of the nerve growth factor via the TrkA/Erk1/2 pathway. Not 
only does $H$. erinaceus induce nerve growth factors or nerve regeneration, but it has also been shown to improve digestive functioning and effect relief from gastritis while providing immune-support, such as anti-inflammatory and anti-oxidant activities. Several studies have demonstrated that hericenones such as hericenone A (19) and erinacines (erinacine C (20) (Fig. 6) induce the synthesis of nerve growth factors in vivo and in vitro (Thongbai et al. 2015). Wittstein et al. (2016) discovered corallocins A-C, a nerve growth and brain-derived neurotrophic factor inducing metabolites, from the related Hericium coralloides in cell based bioassays, while Rupcic et al. (2018) discovered two new erinacine derivatives from mycelial cultures of $H$. erinaceus and $H$. flagellum. Additional studies have revealed neuroactive activities in extracts from medicinal mushrooms that induce nerve growth factors, but the active principles remain to be identified. For instance, the aqueous sclerotium extract from the Malaysian medicinal mushroom, Lignosus rhinocerotis, contained neuroactive compounds that have been demonstrated to stimulate neurite outgrowth in vitro (Eik et al. 2012). Similarly, aqueous extracts of fruiting bodies of species referred to as "Ganoderma lucidum" (questionable identification because the report was from Asia where this species does not actually occur) and G. neo-japonicum were also effective at stimulating neurite outgrowth (Seow et al. 2013). Pleurotus giganteus was found to contain a high concentration of uridine, which has also shown nutritional outgrowth stimulatory effects (Phan et al. 2012). These results may give rise to a more systematic study of the phenomenon in the future.

The family Hericicaceae comprises other genera and species, such as Laxitextum incrustatum Mudalungu et al. (2016) and Dentipellis fragilis (Mitschke 2017), which were proven to contain compounds of the erinacine type; these cyathane terpenoids may constitute family-specific markers. On the other hand, Bai et al. (2015) have reported cyathane diterpenoids as nerve growth factor enhancers from cultures of a Cyathus species, and even the cyathanes from the fruit bodies of the mycorrhizal basidiomycetes of the genus Sarcodon seem to have similar effects (Cao et al. 2018). It should therefore be worthwhile to study genera that are known to produce cyathanes, as well as representatives of other mushroom genera related to Hericium, including Amylosporus and Wrightoporia, for such phenomena. This examination may lead to the discovery of additional novel neurotropic compounds. Reliable screening systems as well as genetic models of human neurodegenerative diseases are now available for in vivo cell biological analysis of disease progression and intervention.

While the mode of action of the hericenones, corallocins and erinacines remains to be clarified, a very important drug that constitutes a mimetic of a fungal metabolite should be mentioned in this context. Fingolimod (21) is a product of total synthesis that has been discovered during the course of a mimetic synthesis program that used as template myriocin (22), a compound produced by the insect associated ascomycete Isaria sinclairii (Strader et al. 2011) (Fig. 6). Fingolimod (sold under the brand Gilenya), is a potent immunosuppressant that was approved in 2010 by the U.S. Federal Drug Association as a new treatment for multiple sclerosis. Fingolimod is phosphorylated in vivo by sphingosine kinase 2 , and the resulting metabolite binds to the extracellular $\mathrm{G}$ protein-coupled receptors, sphingosine 1-phosphates. This prevents the release of lymphocytes from lymphoid tissue and therefore can suppress the immune system. Aside from its proven effects against the symptoms of multiple sclerosis, the compound can also potentially be used in the therapy of cancer and during organ transplants. Likewise, additional potential usages for the erinacines and hericenones may become conceivable once the biochemical and molecular mechanisms by which they exert their activities in biological systems have been elucidated.
Fig. 6 Chemical structures of neurotrophic compounds from Hericium, as well as myriocin and its synthetic analogue fingolimod
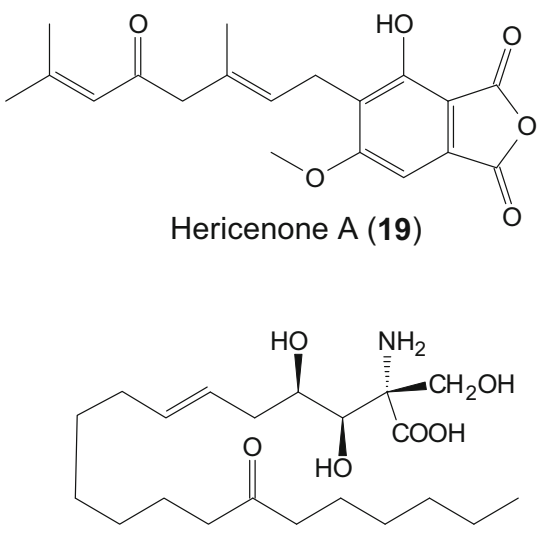

Myriocin (22)

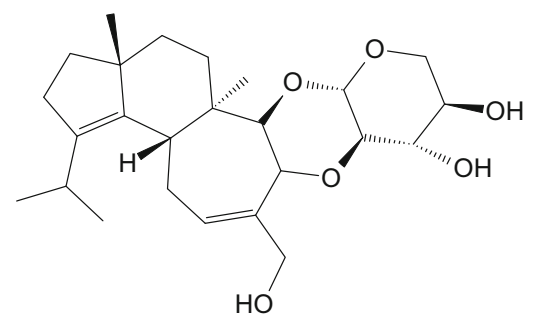

Erinacine C (20)<smiles>CCCCCCCCc1ccc(CCC(N)(O)CO)cc1</smiles> 


\section{Fungi in Traditional Chinese Medicine}

Traditional Chinese Medicine (TCM) has been used for thousands of years, during which time traditional knowledge originating in ancient China has been gradually developed further and distributed to Japan and other Asian countries. TCM relies heavily on the use of natural remedies including plants, animals, and even minerals to cure various diseases. However, in spite of the fact that the term "herbal medicine" is widely used to describe TCM and other ethnobotanical approaches for disease therapy, several mushrooms are commonly used as important ingredients. Furthermore, since fungal taxonomy has yet to be globally harmonized, there is considerable confusion concerning the identity of these important traditional "herbal" remedies. A compilation of the most important TCM mushrooms is therefore provided in Table 2, and we will henceforth refer to the species according to their scientific names as given in this table, regardless of whether they have been or are still being referred to by incorrect or ambiguous names. For instance, we will refer to
"Ganoderma lucidum" as G. lingzhi, regardless of whether the authors of the respective cited papers had already adopted the modern taxonomy.

TCM has evolved through a combination of theory and practical experience of Chinese healers over many centuries. The first recorded use of Chinese medicine was during the Shang Dynasty in the eleventh century BC (1100-1001 BC), where even Ganoderma was recorded in detail and for the first time. However, the first comprehensive description of Chinese herbal remedies and their medicinal value was published in "Shennong Bencaojing ( $\square \square \square \square \square$ )," during the Han dynasty (206 BC-220 AD). Later, another very comprehensive account of Chinese herbology, known as the "Bencao Gangmu ( $\square \square \square \square$ )" or "Compendium of Materia Medica" (Sanodiya et al. 2009) was compiled by Li Shizhen $(1518 \sim 1593)$ during the Ming Dynasty (1368-1644). In 1987, Chinese scientists compiled a list of 272 medicinal mushroom species (Ying et al. 1987). Later, this number increased to 799 species, 500 thereof with antitumor effects (Wu et al. 2013), and we estimate that about 850 species are presently being used as

Table 2 The correct scientific terminology of some important medicinal mushrooms used in traditional Chinese medicine

\begin{tabular}{|c|c|c|}
\hline Current name & Important synonyms/misapplied names & Literature \\
\hline $\begin{array}{l}\text { Agaricus subrufescens } \\
\text { (巴西磨菇: Bā xī mó gū) }\end{array}$ & $\begin{array}{l}\text { Agaricus blazei (ss. Heinemann) (s. auct.); A. } \\
\text { rufotegulis (s. auct.); Agaricus brasiliensis }\end{array}$ & Kerrigan (2005) \\
\hline $\begin{array}{l}\text { Cordyceps militaris } \\
\text { (虫草花: Chóng cǎo huā) }\end{array}$ & $\begin{array}{l}\text { Clavaria militaris L. (s. auct.); Torrubia militaris } \\
\text { (s. auct.) }\end{array}$ & Shrestha et al. (2010), Sung et al. (2007), Baroni (2017) \\
\hline $\begin{array}{l}\text { Ganoderma lingzhi } \\
\text { (靈芝: Líng zhī) }\end{array}$ & $\begin{array}{l}\text { Ganoderma lucidum (s. auct.); G. sichuanense (s. } \\
\text { auct.) }\end{array}$ & Cao et al. (2012), Dai et al. (2017) \\
\hline $\begin{array}{l}\text { Ganoderma sinense } \\
\text { (紫芝: Zǐ zhī) }\end{array}$ & Ganoderma formosanum (s. auct.) & Wang et al. (2005a), Thawthong et al. (2017) \\
\hline $\begin{array}{l}\text { Grifola frondosa } \\
\text { (灰树花: Hūi shù huā) }\end{array}$ & $\begin{array}{l}\text { Boletus frondosus (s. auct.) Polyporus frondosus } \\
\text { (s. auct.) }\end{array}$ & Corner (1989), Shen et al. 2017) \\
\hline $\begin{array}{l}\text { Hericium erinaceus } \\
\text { (猴头菇: Hóu tóu gū) }\end{array}$ & Hydnum erinaceus (s. auct.) & Zhou et al. (2011), Thongbai et al. (2015) \\
\hline $\begin{array}{l}\text { Lentinula edodes } \\
\text { (香葍: xiāng xùn) }\end{array}$ & Lentinus edodes (s. auct.) & $\begin{array}{l}\text { Molina et al. (1992), Hibbett and Vilgalys (1993), García- } \\
\text { Mena et al. (2007), Vetchinkina et al. (2008) }\end{array}$ \\
\hline $\begin{array}{l}\text { Lycoperdon pyriforme } \\
\text { (梨形马勃: Lí xíng mă bó, or } \\
\text { 马勃属: Mǎ bó shǔ) }\end{array}$ & Morganella pyriformis (s. auct.) & Krüger and Kreisel (2003), Larsson and Jeppson (2008) \\
\hline $\begin{array}{l}\text { Ophiocordyceps sinensis } \\
\text { (冬虫夏草: Dōng chóng xià } \\
\text { căo) }\end{array}$ & Cordyceps sinensis (s. auct.) & Shrestha et al. (2010), Zhang et al. (2012) \\
\hline $\begin{array}{l}\text { Trametes versicolor } \\
\text { (雲芝: Yún zhī) }\end{array}$ & $\begin{array}{l}\text { Coriolus versicolor (s. auct.); Polyporus versicolor } \\
\text { (s. auct.) }\end{array}$ & $\begin{array}{l}\text { Tomšovský and Homolka (2004), Justo and Hibbett (2011), } \\
\text { Wan (2013) }\end{array}$ \\
\hline $\begin{array}{l}\text { Wolfiporia cocos } \\
\text { (茯苓: Fú líng) }\end{array}$ & Poria $\operatorname{cocos}$ (s. auct.) & Kubo et al. (2006), Dai et al. (2009) \\
\hline $\begin{array}{l}\text { Xylaria nigripes } \\
\text { (黑柄炭角菌: Hēi b̌̌ng tàn } \\
\text { jiăo jūn) }\end{array}$ & Pseudoxylaria nigripes (s. auct.) & Rogers et al. (2005) \\
\hline
\end{tabular}


Fig. 7 Different dried Ganoderma products sold in a Traditional Chinese Medicine pharmacy market, Kunming Province, China
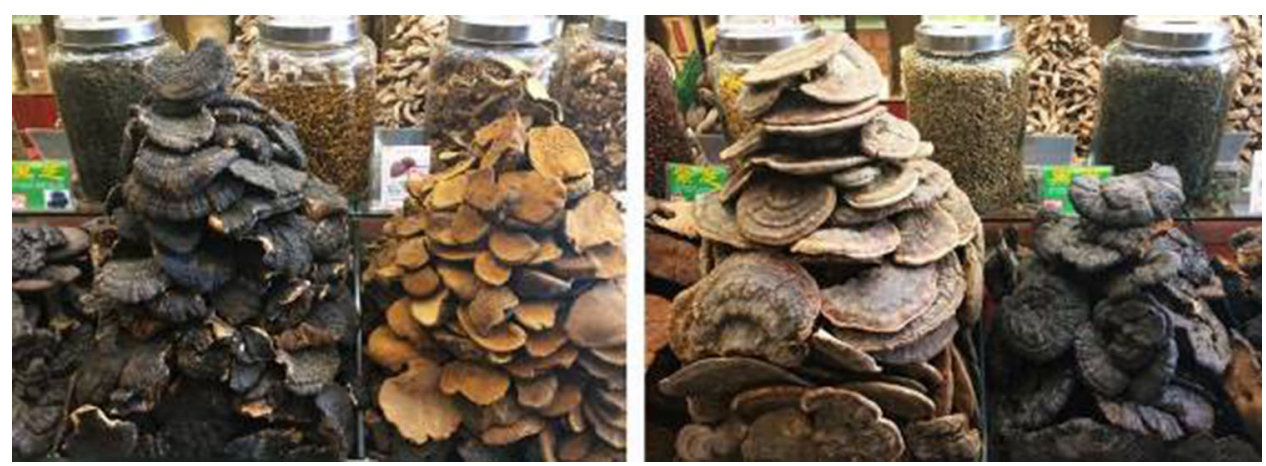

remedies in China. TCM has adopted a more holistic philosophy when compared with Western medicine (Wang et al. 2017). Chinese medicinal mushrooms have been used since the beginning of human history as a "protector herb" in order to maintain and enhance good health.

Whereas the majority of the TCM ingredients are derived from plants, several macrofungi have been included in the formulas. Some macrofungi are frequently included in TCM medicine formulas and are also very commonly sold in the medicinal or local markets (Fig. 7). The most important species and species groups are compiled in Table 2. Notably, some, such as Ganoderma lingzhi and Wolfiporia cocos, are very expensive, while others such as Ophiocordyceps sinensis have become rare in nature (Sung et al. 2007; Zhang et al. 2012; Xiang et al. 2014; Hapuarachchi et al. 2018, 2019).

Ganoderma lingzhi (Lingzhi) contains both complex, high and low molecular weight natural bioactive compounds, which are mostly triterpenoids (Sandargo et al. 2019a). Bioactive compounds have shown in vitro activities against cancer cells, although none have been regarded as effective enough to enter clinical development as an ethical anticancer drug. Extracts of mycelial cultures and fruiting bodies of Ophiocordyceps sinensis and Cordyceps militaris primarily contain cordycepin, a derivative of nucleoside adenosine, with dimethylguanosine and iso-sinensetin, and showed moderate anticancer and antiproliferative effects in vitro (Wong et al. 2010). Several bioactive compounds derived from mushrooms used in TCM were identified by bioactivity-guided fractionation. On the other hand, the potential therapeutic value of these fungal TCM products for the treatment of various diseases (e.g. cancer, diabetes, cardiovascular and neurodegenerative diseases) is even supported by clinical studies (Table 3).

There has been intensive debate over the utility of TCM for modern healthcare. Many clinical scientists have doubted the utility of the classical TCM remedies because of their inaccurate descriptions, as well as the different philosophies regarding their therapeutic application. In fact, therapy for diseases in the Western world is mainly based on the application of single substances that have strong and selective biological and pharmacological activities, and must undergo very thorough clinical trials before they can be applied as therapeutic drugs. TCM, by contrast, often uses mixtures of preparations made from several plants, fungi and other organisms, each of which may contain dozens if not hundreds of different compounds. Therefore TCM does not really fit into the modern healthcare system ( $\mathrm{Fu}$ and $\mathrm{Yu}$ 2005). However, the example of artimisinin shows that compounds from TCM can be very useful as remedies for diseases that have an unmet medical need. This terpenoid was originally found in the asteraceous traditional Chinese medicine plant Artemisia апnиа; a semisynthetic derivative, artesunate, is now on the market as a last generation antimalaria drug (the inventor received the Nobel Prize for Medicine in 2015). This example shows that it is worthwhile to study the ingredients of traditional Chinese medicine plants using modern drug research methods in order to discover novel therapeutic agents. Several examples of beneficial compounds from mushrooms have found their way into exploratory drug research projects (Grothe et al. 2011).

In addition, the recent integration of TCM and modern medicine has begun to solve multiple worldwide health problems. Many databases were evaluated to advance scientific formulations, chemical analysis, potential approaches, and other health targets. The World Health Organization (WHO) has also released a standard series for developing traditional medicines across the world, including medicinal mushrooms (Tang et al. 2018). The discovery of biologically active compounds from medicinal mushrooms can impact the direction of future medical development, and also has broad market prospects in North America, Europe and other developed economies (Lee et al. 2012; Wang et al. 2017). This may also increase international exchange, leading to the unprecedented development of Chinese medicine in the western world (Zhu 2018). 
Table 3 Some important medicinal mushrooms used in traditional Chinese medicine

\begin{tabular}{|c|c|c|c|}
\hline Fungal species & Therapeutic use (diseases) & Instructions & References \\
\hline $\begin{array}{l}\text { Agaricus } \\
\text { subrufescens }\end{array}$ & $\begin{array}{l}\text { Anticancer, anti-inflammatory, anti-allergic, } \\
\text { hepatoprotective, diabetic, arteriosclerosis and (treat } \\
\text { kidney disease, bowel disease, asthma, diabetes and } \\
\text { high blood sugar levels) }\end{array}$ & $\mathrm{P}, \mathrm{S}$, and $\mathrm{T}$ & $\begin{array}{l}\text { Grothe et al. (2011), Kerrigan (2005), Jeong } \\
\text { et al. (2010), Lee et al. (2012) }\end{array}$ \\
\hline $\begin{array}{c}\text { Cordyceps } \\
\text { militaris }\end{array}$ & $\begin{array}{l}\text { Anticancer, antioxidant, antibacterial, antifungal, } \\
\text { antiviral, antihypertensive, diabetic, anti-fibrotic, anti- } \\
\text { angiogenesis, and hepatoprotective (treating kidney } \\
\text { disease, lung fibrosis, high blood pressure, high blood } \\
\text { sugar levels, and strengthen sexual function) }\end{array}$ & $\mathrm{B}, \mathrm{M}$, and $\mathrm{S}$ & Lee et al. (2012), Xiang et al. (2014) \\
\hline $\begin{array}{l}\text { Ganoderma } \\
\text { lingzhi }\end{array}$ & $\begin{array}{l}\text { Immunostimmulatory, anticancer, anti-inflammatory, } \\
\text { antiviral, antioxidant, antibacterial, antifungal, anti- } \\
\text { hypotensive, anti-metastatic effect and diabetic (treat } \\
\text { kidney, liver and lung diseases, asthma or bronchial, } \\
\text { insomnia, neurasthenia, and strengthen sexual function) }\end{array}$ & $\begin{array}{l}\mathrm{B}, \mathrm{P}, \mathrm{S}, \mathrm{SP} \\
\quad \text { and } \mathrm{T}\end{array}$ & $\begin{array}{l}\text { Liu et al. (2002), Richter et al. (2015), Qiao et al } \\
\text { (2005), Cheng et al. (2010), Teng et al. } \\
\text { (2011, 2012), Lee et al. (2012), Hapuarachchi } \\
\text { et al. (2014) }\end{array}$ \\
\hline $\begin{array}{l}\text { Ganoderma } \\
\text { sinense }\end{array}$ & $\begin{array}{l}\text { Immunostimmulatory, anticancer, antiviral, anti- } \\
\text { inflammatory, antioxidant, antibacterial and antifungal } \\
\text { (treating liver, chronic coughs, asthma and leukopenia) }\end{array}$ & $\begin{array}{l}\mathrm{B}, \mathrm{P}, \mathrm{S}, \mathrm{SP} \\
\text { and } \mathrm{T}\end{array}$ & $\begin{array}{l}\text { Richter et al. (2015), Teng et al. (2011, 2012), } \\
\text { Jiang et al. (2017) }\end{array}$ \\
\hline Grifola frondosa & $\begin{array}{l}\text { Anticancer, antiviral, anti-inflammatory, anti- } \\
\text { hypotensive, hepatoprotective, and diabetic (treat liver, } \\
\text { therapy of HIV, low blood pressure, and high blood } \\
\text { sugar levels) }\end{array}$ & $\mathrm{S}$ and $\mathrm{T}$ & Chien et al. (2017), Ma et al. (2015a, b) \\
\hline $\begin{array}{l}\text { Hericium } \\
\text { erinaceus }\end{array}$ & $\begin{array}{l}\text { Anticancer, neuroprotective hemostatic (used to } \\
\text { treatment Alzheimer's and Parkinson's disease) }\end{array}$ & $\mathrm{P}, \mathrm{S}$, and $\mathrm{T}$ & $\begin{array}{l}\text { Salmon (2012), Thongbai et al. (2015), Cheng } \\
\text { et al. (2016), Yang et al. (2016), Zhang et al. } \\
\text { (2017a) }\end{array}$ \\
\hline Lentinula edodes & $\begin{array}{l}\text { Anticancer, antiviral, antibacterial, anti-inflammatory, } \\
\text { and antihypertensive (treatment of high blood pressure) }\end{array}$ & $\begin{array}{l}\mathrm{B}, \mathrm{M}, \mathrm{S} \text {, and } \\
\mathrm{T}\end{array}$ & $\begin{array}{l}\text { Lee et al. (2012), Rincão et al. (2012), Lin et al } \\
\text { (2015) }\end{array}$ \\
\hline $\begin{array}{l}\text { Lycoperdon } \\
\text { pyriforme }\end{array}$ & $\begin{array}{l}\text { Antibacterial, anti-inflammatory, antitumor, (treatment of } \\
\text { pharyngitis; hemostasis) }\end{array}$ & $\begin{array}{l}\text { M (young } \\
\text { fruiting } \\
\text { body), and } \\
\text { SP }\end{array}$ & Akatin (2013), Qian et al. (2018) \\
\hline $\begin{array}{l}\text { Ophiocordyceps } \\
\text { sinensis }\end{array}$ & $\begin{array}{l}\text { Anticancer, antiviral, anti-inflammatory, antioxidant, } \\
\text { antibacterial, antifungal, anti-angiogenetic, anti- } \\
\text { proliferative, hypoglycaemic, hypolipidaemic, } \\
\text { renoprotective, hepatoprotective (treat heart, lung and } \\
\text { kidney diseases, high blood sugar levels and strengthen } \\
\text { sexual function) }\end{array}$ & $\begin{array}{l}\mathrm{M}, \mathrm{P}, \mathrm{S} \text { and } \\
\mathrm{T}\end{array}$ & $\begin{array}{l}\text { Akihisa et al. (2009), Jeon et al. (2011), Lee } \\
\text { et al. (2012), Xiang et al. (2014) }\end{array}$ \\
\hline $\begin{array}{l}\text { Trametes } \\
\text { versicolor }\end{array}$ & $\begin{array}{l}\text { Antitumor, antiviral, anti-inflammatory, antimalarial } \\
\text { activity, diabetic, and hepatitis (treat kidney disease, } \\
\text { diabetes, malaria and HIV) }\end{array}$ & $\mathrm{B}, \mathrm{C}$ and $\mathrm{T}$ & Fu and Yu (2005), Adekunle et al. (2016) \\
\hline Wolfiporia cocos & $\begin{array}{l}\text { Immunostimmulatory, antiviral, anti-inflammatory, } \\
\text { antiasthmatic, hepatoprotective, anti-parasitic } \\
\text { (treatment of liver and lung disease, asthma or } \\
\text { bronchial) }\end{array}$ & $\mathrm{C}, \mathrm{P}$ and $\mathrm{T}$ & $\begin{array}{l}\text { Wang et al. (2013b), Wei et al. (2016), Zhu et al } \\
\text { (2018) }\end{array}$ \\
\hline Xylaria nigripes & $\begin{array}{l}\text { Anticancer, antiviral, anti-inflammatory, hypoglycaemic, } \\
\text { hepatoprotective (treating kidney disease, } \\
\text { gynecological diseases, and therapy of insomnia) }\end{array}$ & $\mathrm{B}, \mathrm{C}$ and $\mathrm{T}$ & Fu and Yu (2005), Peng et al. (2015b) \\
\hline
\end{tabular}

$B$ boiled with herbs, $M$ meals/dishes, $S$ soup, $S P$ spore, $P$ powder, $T$ tablet

Chinese medicines have been utilized within several alternative medicinal practices to introduce positive changes in order to provide better contributions for health care while developing future medicinal products for diseases and for use in therapy (Lin et al. 2015; Han et al. 2017). However, there are challenges to the development of TCM products, due in part to the nature of the therapeutic potential and the fact that the mechanisms of action are often unclear. Furthermore, the materials used often do not meet the requirements of quality control and standardisation (Wen et al. 2017), and hence they are difficult to register as prescription drugs. Many materials have not 
been tested thoroughly to the standards of western medicine and can therefore only be sold as over-the-counter drugs or even neutraceuticals. Many challenges still stand in the way towards international collaborative exchange for opportunities to develop TCM, thereby advancing it at the global level (Chen et al. 2016). The use of mushrooms is advantageous when compared with the plants used in TCM, since mushrooms can be produced biotechnologically under controlled conditions and with standards, making them easier to work with than species that have to be grown and harvested in nature. In conclusion, to achieve the global acceptance of TCM products, it will be necessary to conduct clinical trials based on high quality standardised materials.

\section{Cardiovascular disease control by fungi}

Cardiovascular diseases include diseases of the heart, vascular diseases of the brain, and diseases of blood vessels (Mendis et al. 2011). Elevated levels of plasma cholesterol are responsible for these diseases, as they play a major role in atherosclerosis, the clogging or hardening of arteries caused by accumulations of fatty deposits (usually cholesterol) (Miller 2001). These diseases were responsible for 17.3 million deaths per year in 2015, and are the leading source of deaths worldwide (GBD 2015). Inhibition of de novo synthesis of cholesterol was demonstrated to be an effective method for reducing plasma cholesterol levels (Miller 2001). The rate determining step is the reduction of 3-hydroxy-3-methylglutaryl-coenzyme A (HMG-CoA) to mevalonate by HMG-CoA reductase; hence selective inhibition of the latter enzyme, can reduce the synthesis of cholesterol (Brown et al. 1976; Endo et al. 1976). The most important class of HMG-CoA reductase inhibitors are the statins (Fig. 8), which are derived from fungal natural products and contain two types of structures moieties, a hexahydro-naphthalene system and a $\beta$-hydroxylactone system. The statins are also the best-selling class of pharmaceutical drugs, with an annual turnover in the range of US\$50 billion. Therefore, the most important compounds of this type are treated here.

Fig. 8 Chemical structures of statins with cholesterollowering properties
Brown et al. (1978) isolated compactin (22) (also known as mevastatin and ML-236B) from Penicillium brevicompactum as an antibiotic, but its hypocholesterolemic activity was later revealed by Endo et al. (1976), who used the name "ML-236B". Later another HMG-CoA reductase inhibitor named mevinolin (23) was isolated from Aspergillus terreus by Alberts et al. (1980). The first statin drug approved by the United States Food and Drug Administration was lovastatin (24) in 1987 (Jahromi et al. 2012). Although lovastatin is produced by several species of Penicillium (Endo et al. 1976), Monascus species (Miyake et al. 2006; Sayyad et al. 2007), Doratomyces, Gymnoascus, Hypomyces, Phoma and Trichoderma, the commercialized product is derived from Aspergillus terreus (Jahromi et al. 2012). Another statin containing a product called Xuezhikang or "red yeast rice extract", produced by the fermentation of Monascus spp., has been widely used in China for centuries for treating circulatory disorders. The low density lipoprotein lowering ability of "red yeast rice extract" is clinically proven and contains lovastatin (Lu et al. 2008).

Solid state fermentation, or submerged cultures, can be used for the production of lovastatin (Ruddiman and Thomson 2001; Lai et al. 2003; Suryanarayan 2003; Wei et al. 2007; Jaivel and Marimuthu 2010; Pansuriya and Singhal 2010; Jahromi et al. 2012), but production is significantly higher in the former (Jaivel and Marimuthu 2010; Jahromi et al. 2012). Sorghum grain, wheat bran, rice and corn are used as substrates for solid state fermentation (Wei et al. 2007; Jaivel and Marimuthu 2010; Jahromi et al. 2012).

Basidiomycetes are a good source of nutrient supplement for humans. Certain molecules in mushrooms can modify cholesterol absorption, metabolism, and also modulate the gene expression related to cholesterol homeostasis (Gil-Ramírez et al. 2016). Grifola frondosa, Hypsizigus marmoreus and Pleurotus ostreatus were able to differentially modulate the gene expression patterns of mice livers (Gil-Ramírez et al. 2016). Of the biologically active compounds from fungi that can reduce the amounts of cholesterol in the blood, the most studied are ergosterol derivatives (Gil-Ramírez et al. 2016). The cholesterol-

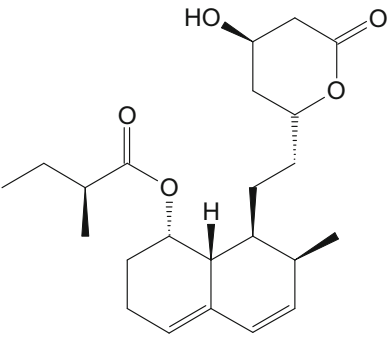

Compactin (22)

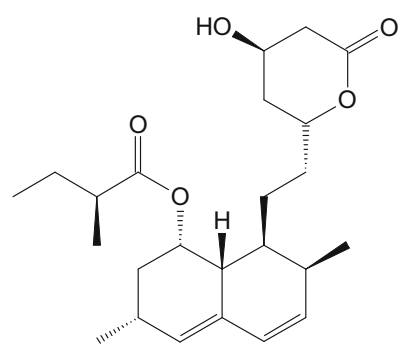

Mevinolin (23)

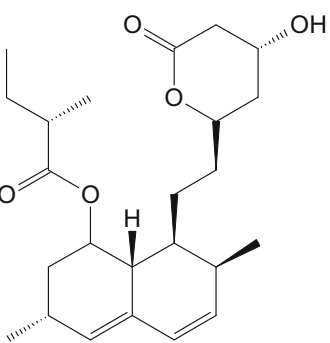

Lovastatin (24) 
lowering properties are mainly caused by the structural similarity with cholesterol (Gil-Ramírez et al. 2016). Further, the biological activity of $\beta$-glucans and chitin may be due to their binding abilities to cholesterol receptors (GilRamírez et al. 2016).

Francia et al. (1999) recorded 16 species of edible mushrooms with biological activities against cardiovascular disease. Species of the genera Auricularia (Fan et al. 1989), Ganoderma (Kabir et al. 1988), Grifola (Kubo and Namba 1997), Pleurotus (Bobeck et al. 1991) and Tremella (Cheung 1996) have been reported to contain cholesterollowering compounds. Ophiocordyceps sinensis has also been shown to reduce total cholesterol levels, which has been attributed to the fact that it contains polysaccharide "CS-F30" composed of galactose, glucose and mannose (Kiho et al. 1996). For instance, low density lipoprotein cholesterol levels were reported to be reduced by Auricularia auricula-judae (Fan et al. 1989) and Tremella fuciformis (Cheung 1996), and triglyceride levels were reported to be reduced by Grifola frondosa (Kubo and Namba 1997), Lentinula edodes (Kabir and Kimura 1989) and Ophiocordyceps sinensis (Kiho et al. 1996).

\section{Antiviral agents}

Health and mortality-debilitating diseases caused by viruses continue to cause serious global epidemics, especially in cases where vaccines and antiviral chemotherapies are insufficient or not available. The current state of virusrelated pandemics is also significantly limiting drug efficacy by the emergence of drug-resistant strains. Hence, there is an urgent need to identify and develop natural product-inspired drug leads that could help control viral infections. A plethora of potentially active natural products have been isolated from fungi and screened for antiviral activity, even though none of them has reached the market yet. This entry focuses on natural products exhibiting potent activity on selected human pathogenic viruses, such as the human immunodeficiency virus (HIV), influenza virus, herpes simplex virus (HSV), hepatitis virus and other human pathogenic viruses such as enterovirus-71, and respiratory syncytial virus (RSV).

Human Immunodeficiency Virus (HIV) inhibitory natural products from fungi

A comprehensive review of the literature identifies three main targets for anti-HIV drug discovery: virus entry, reverse transcription and integration.

The entry of HIV involves interactions with proteins and is a target for the discovery of new viral entry blockers. Examples of a few discoveries are provided. A bis-indolyl quinone, hinnuliquinone (25) (Fig. 9) from an unknown fungus isolated from Quercus coccifera, inhibited wildtype and clinically-resistant HIV-1 protease. HIV-1 protease is a key enzyme involved in the replication and
Fig. 9 Chemical structures of fungal metabolites that were reported to possess antiviral activities

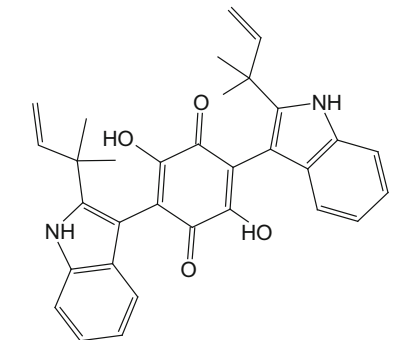

Integrastatin $\mathrm{A}(\mathbf{2 7})$

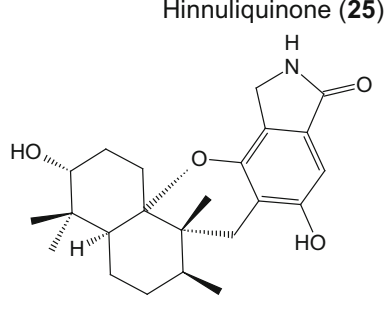

(c)

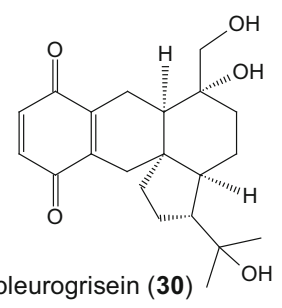

Vanitaracin A (29)

4-hydroxypleurogrisein (30)
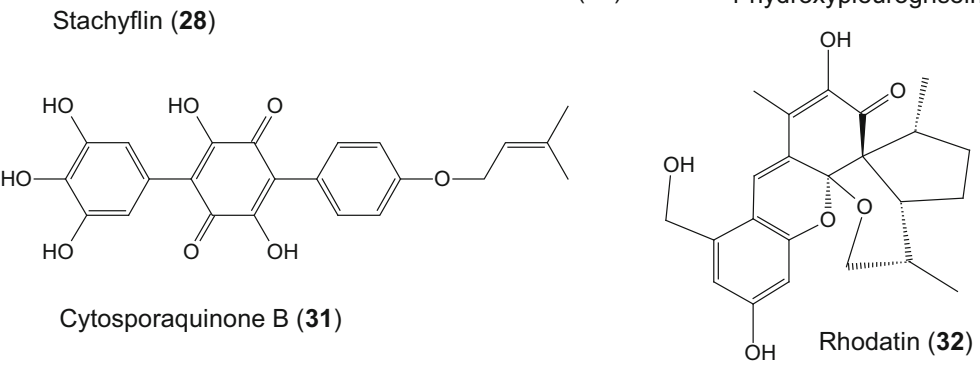
maturation of the HIV-1 virus (Singh et al. 2004). Altertoxins I-III and V, oxidized perylenes from Alternaria tenuissima, inhibited HIV-1 replication at micromolar concentrations (Bashyal et al. 2014). The dimeric tetrahydroxanthone, penicillixanthone A from Aspergillus fumigatus displayed strong anti-HIV activity by inhibiting CCR5-tropic HIV-1 SF162 and CXCR4-tropic HIV NL4-3 (Tan et al. 2017). A marine-derived A. niger produced malformin $\mathrm{C}$, which exhibited a very strong anti-HIV-1 activity (Zhou et al. 2015a). An endophytic Aspergillus sp. CPCC 400735 produced three phenalone and cytochalasin derivatives also showing anti-HIV activity (Pang et al. 2017). Concentricolide from "Daldinia concentrica" (taxonomy doubtful since this species does not occur in China according to the world monograph by Stadler et al. 2014) inhibited HIV-1 by induction of cytopathic effects (Fang and Liu 2009). Novel sesquiterpenoids from Paraconiothyrium brasiliense showed moderate anti-HIV-1 replication in C8166 cells (Liu et al. 2010b). The pupukeanane sesquiterpenoid chloropupukeannolide A from Pestalotiopsis fici showed significant anti-HIV-1 activity (Liu et al. 2010c). The cytochalasan perconiasin $\mathrm{J}$ and the meroterpenoid periconone B from Periconia sp. displayed moderate anti-HIV activity (Liu et al. 2016, 2017b). The farnesylated isoindolinones stachybotrysams $\mathrm{A}-\mathrm{C}$ and the phenylspirodrimane derivatives stachbotrysin $A$ and $G$ from Stachybotrys chartatum displayed moderate anti-HIV activity (Zhao et al. 2017a, b).

The three consecutive functions controlled by HIV reverse transcriptase are: RNA reverse transcription to DNA, degradation of RNA template by RNase $\mathrm{H}$, and duplication of the remaining DNA strand. Inhibition of these processes is important for the discovery of anti-HIV drugs. Stachybosin D (26) (Fig. 9), a phenylspirodrimane metabolite from a sponge-derived isolate of Stachybotrys chartarum, showed inhibitory effects on HIV-1 replication by targeting reverse transcriptase. It was able to inhibit NNRTIs-resistant strains and wild-type HIV-1 (Ma et al. 2013).

Integrase is the only protein encoded by HIV-1, aside from the enzymes protease and reverse transcriptase. Singh et al. (1998, 2002a, b, 2003a, b, c) described several compounds with inhibitory activity against integrase from various fungal species. Accordingly, equisetin and phomasetin from Fusarium heterosporum and Phoma sp., respectively Singh et al. 1998); integracins (Integrastatin A (27)) from Cytonaema sp. (Singh et al. 2002a); integrastatins (from an unidentified fungus; cf. Singh et al. 2002b); epiphiobolins C and K from "Neosartorya"; i.e., Aspergillus sp.; 8-O-methylanthragallol from Cylindrocarpon ianthothele; hispidin and caffeic acid from Inonotus tamaricis; 3-hydroxyterphenyllin from Aspergillus candidus (Singh et al. 2003a); naphtho- $\gamma$-pyrones from
Fusarium sp. (Singh et al. 2003b); and xanthoviridicatins from Penicillium chrysogenum (Singh et al. 2003c) all showed low micromolar inhibition against the cleavage reaction of HIV integrase. Funalenone from Penicillium sp. FKI-1463 also had the same effect (Shiomi et al. 2005).

Influenza virus inhibitory natural products from fungi The H1N1 and H3N2 viruses are among the targets of natural products of fungal origin with anti-influenza activity. The terpenoid stachyflin (28), isolated from a marine-derived isolate of Stachybotrys showed modest activity against the influenza $\mathrm{A}$ virus (H1N1) with an $\mathrm{IC}_{50}$ of $3 \times 10^{-3} \mu \mathrm{M}$ (Minagawa et al. 2002). The $\gamma$-pyrone isoasteltoxin from Aspergillus ochraceopetaliformis showed low micromolar activity $\left(\mathrm{IC}_{50}=0.23 \mu \mathrm{M}\right)$ against both influenza viruses (Wang et al. 2016). Another Aspergillus sp., strain produced the $\gamma$-pyrone derivative asteltoxin $\mathrm{E}$ with $\mathrm{IC}_{50}$ values of 6.2 and $3.5 \mu \mathrm{M}$ against H1N1 and H3N2, respectively (Tian et al. 2016). 33-Hydroxysterol from Pestalotiopsis sp. (Sun et al. 2014) and chermesinone from Nigrospora sp. (Zhang et al. 2016b) also had moderate inhibitory effects. Aureonitol, a metabolite of Gliocladium spp. inhibited influenza A and B virus replication with an $\mathrm{EC}_{50}$ of $100 \mathrm{nM}$ against $\mathrm{H} 3 \mathrm{~N} 2$ via suppression of influenza hemagglutination, while significantly impairing viral adsorption (Sacramento et al. 2015).

\section{Herpes Simplex virus (HSV) inhibitory natural prod- ucts from fungi}

The impotant human pathogenic viruses HSV-1 and HSV-2 were also subject of sceening programs of fungal metabolite libraries, even though up to date no drug could be discovered that would match the activity of the market standard. Coccoquinone, an anthraquinone from Aspergillus versicolor, demonstrated an $\mathrm{IC}_{50}$ of $3 \mu \mathrm{M}$ against HSV1 (Huang et al. 2017a, b). Five lipopeptides from the marine-derived fungus Scytalidium sp. showed moderate anti-HSV-1 and anti-HSV-2 activities in a dose- and timedependent pattern (Rowley et al. 2003). The diphenyl ether glycoside cordyol C from Cordyceps sp. BCC 186 exhibited significant anti-HSV-1 activity with an $\mathrm{IC}_{50}$ value of $1.3 \mu \mathrm{g} / \mathrm{ml}$ (Bunyapaiboonsri et al. 2011).

Hepatitis virus inhibitory natural products from fungi One novel tricyclic polyketide derived from a collection of fungal-derived compounds, vanitaracin A (29), was reported to inhibit viral entry process with an $\mathrm{IC}_{50}$ value of $0.6 \mu \mathrm{M}$ and good selectivity. It was observed to directly interact with the HBV entry receptor correlated to hepatitis $\mathrm{D}$ virus and impaired viral bile acid transport pathway. This compound also inhibited all HBV genotypes (A-D) (Kaneko et al. 2015). The anthraquinone metabolite, 2'R-1hydroxyisorhodoptilonmetrin, showed better anti-hepatitis B virus activity as compared to the positive drug control, 
lamivudine (Jin et al. 2018). The epipolythiodioxopiperazine derivative, $11^{\prime}$-deoxyverticillin A, showed antiviral activity by decreasing HBV-X replication through inhibition of Akt activity or depletion of the autophagic genes, LC3 and p62 (Wu et al. 2015a). Sandargo et al. (2018) and Narmani et al. (2019) have recently discovered additional anti-HCV agents like 4-hydroxypleurogrisein (30) from the nematode trapping basidiomycete Hohenbuehelia grisea and cytosporaquinone B (31) from an Iranian phytopathogen belonging to the genus Cytospora. Recently Sandargo et al. (2019b) reported the meroterpenoid rhodatin (32) from cultures of the rare basidiomycete Rhodotus palmatus and also found significant anti-HCV activities for this compound, which features a new carbon skeleton.

In view of the fact that newly arising viral diseases are steadily being reported and they can spread more easily due to gflobalization effects, the search for novel antiviral agents is as of recently gaining importance. Some of the natural products pointed out in this entry may potentially find their way to antiviral drug development in the future. However, the discovery of new chemical derivatives with nanomolar activity and high selectivity indices is still warranted.

\section{Immunosuppressive and immunomodulatory agents from fungi}

Immunosuppression is a form of therapy that prevents the immune system of patients from acting against transplanted tissues and organs; without the availability of immunosuppressive drugs, the progresses made in modern medicine, in particular regarding kidney, heart and liver transplants, would be unthinkable. Furthermore, immunosuppressants are used to control severe manifestations of allergic and autoimmune related diseases. Many of these drugs specifically address certain biochemical pathways that are crucial for the functioning of human defense against alien organisms, such as pathogens, by selectively inhibiting the immunocompetent lymphocytes or signal transduction cascades that regulate the transcription of cytokines. For this reason, patients treated with immunosuppressants often have to be hospitalized and treated in parallel with antibiotics to prevent infection.

Some of the most important immunosuppressive drugs are natural products that are being produced biotechnologically by fermentation of bacteria and fungi. While tacrolimus and sirolimus are derived from actinobacteria and will accordingly not be treated here, cyclosporine (35) and mycophenolate mofetil (33) are fungal metabolites, and the present entry is therefore dedicated to these important molecules (Fig. 10).

Mycophenolic acid (34) (Fig. 10) was the first antibiotic discovered and isolated in crystalline form from fungi, contrary to what is written elsewhere in the literature, where the penicillins are often regarded as the oldest natural antibiotics. Biogenetically, this compound is a meroterpenoid, produced by Penicillium species, including $P$. brevicompactum and $P$. roquefortii, and its biosynthesis has recently been elucidated in the latter species (Del-Cid et al. 2016). For various reasons, the compound never made
Fig. 10 Chemical structures of immunosuppressive drugs derived from fungal fermentation<smiles>COc1c(C)c2c(c(O)c1C/C=C(\C)CCC(=O)OCCN1CCOCC1)C(=O)OC2</smiles>

Mycophenolate mofetil (33)<smiles>COc1c(C)c2c(c(O)c1C/C=C(\C)CCC(=O)O)C(=O)OC2</smiles>

Mycophenolic acid (34)

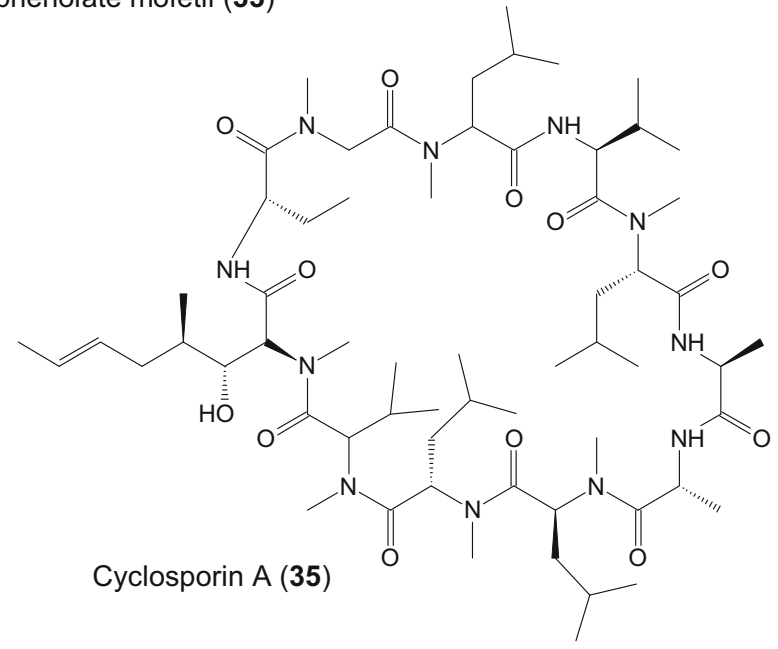


it into clinical development as an antibacterial or antifungal agent (Bentley 2000; Bills and Gloer 2016). Ultimately, its utility as an immunosuppressant became evident, and it is now the active principle of several marketed drugs, such as Myfortic $^{\circledR}$ and CellCept ${ }^{\circledR}$. The compound selectively inhibits inosine monophosphate dehydrogenase (IMPDH), an enzyme that is crucial for the biosynthesis of guanosine nucleotides in mammalian cells. As this enzyme is more essential in the T- and B-lymphocytes than in other cell types, and its isoform in the lymphocytes is more sensitive to mycophenolic acid, the drug has a more potent cytostatic effect on lymphocytes than on other cell types, and thereby suppresses the immune system (Allison and Eugui 2000). Another secondary beneficial effect of mycophenolíc acid is the depletion of tetrahydrobiopterin, which is a co-factor for the inducible nitric oxide synthase (iNOS). Consequently, the administration of mycophenolic acid prevents damage of tissues mediated by peroxynitrite. The drug is mainly being used to prevent organ rejection following transplants, as well as in the therapy of psoriasis (Epinette et al. 1987) and other immunological disorders.

Cyclosporine A (35) (Fig. 10) was first discovered as a mildy active antifungal antibiotic by Dreyfuss et al. (1996), who also gave the first hints as to its immunomodulatory activity. The compound is a nonribosomally biosynthesized peptide derived from fermentation of the ascomycete Tolypocladium inflatum, and its biosynthetic gene cluster was recently elucidated by Bushley et al. (2013). After years of intensive research, it was found that this cyclopeptide has a highly specific biochemical mode of action as it selectively binds to cyclophilin A. This protein is an inhibitor of calcineurin, which is responsible for activation of transcription of the cytokine, interleukine 2. If interleukine 2 is depleted, the immune response of the human body will be suppressed and rejection of transplants can be prevented (Green et al. 1981; Wiesinger and Borel 1980). Therefore, cyclosporine A has become the active ingredient of blockbuster drugs, such as Sandimmune ${ }^{\circledR}$, Neoral ${ }^{\circledR}$ and Restasis ${ }^{\circledR}$ ). In addition to organ transplants, such immunosuppressants can be very useful in the therapy of other diseases, including allergies and neurodegeneration. An example for the latter indication is the synthetic drug, fingolimod (Gilenya ${ }^{\circledR} ; 21$ in Fig. 6), which is described further above in chapter 6 .

In this context, it is worthwhile to note that many fungal metabolites are highly useful in therapy because they show the opposite activities in biological systems, i.e., they boost the immune system and therefore increase the resistance against pathogens or even help to prevent cancer. Striking examples for such molecules are the $\beta$-glucanes and protein-polysaccharide complexes that are being treated elsewhere herein.

\section{Strategies against plant disease}

Fungi are important agents in combating various fungal pests and plant diseases found in greenhouses, the field, and even post-harvest. Fungi also have the potential to be used against certain animal parasites, such as nematodes. In this section, we discuss how fungi are being used to control plant disease, pests, nematodes, and herbicides, as well as their possible future applications.

\section{Biocontrol of plant disease using endophytes}

Fungal pathogens are the chief agent of plant disease, effecting severe agricultural losses worldwide (Hyde et al. 2014; Punja and Raj 2003; Strange and Scott 2005; Horbach et al. 2011). Agrochemicals play a significant role in plant disease management and ensure sustainable and productive agriculture systems. However, the intensive use of chemicals (determined by frequent and high dose of pesticides) has adverse effects on human health, ecosystem functioning, and agricultural sustainability (Anderson et al. 2004; Vinale et al. 2008; Suryanarayanan et al. 2016). Biocontrol is a strategy used to control plant pathogens, resulting in minimal impact to the environment (De Waard et al. 1993; Vinale et al. 2008).

Endophytes reside asymptomatically within a plant for at least part of their life cycle (Carrol 1998; Huang et al. 2009; Sun et al. 2011; Clay et al. 2016). Fungal endophytes can be broadly classified into two groups, the clavicipitaceous (C) and the non-clavicipitaceous (NC). These endophytes are classified based on evolutionary relatedness, taxonomy, host plant range and ecological function (Hyde and Soytong 2008; Rodriguez et al. 2009; O'Hanlon et al. 2012; Santangelo et al. 2015). Clavicipitaceous endophytes, including Atkinsonella, Balansia, Balansiopsis, Echinodothis, EpichloŠ, Myriogenospora and Paraepichlo $\breve{S}$ species are commonly associated with grasses in the family Poaceae and rely on their host throughout their life cycle as mutualist species (Rodriguez et al. 2009; Purahong and Hyde 2011; O'Hanlon et al. 2012; De Silva et al. 2016). Non-clavicipitaceous endophytes, such as Fusarium sp., Colletotrichum sp., Phomopsis sp. and Xylaria sp. are found in most terrestrial plants, and might not inhabit the host plants for their entire life cycle (Rodriguez et al. 2009; Delaye et al. 2013; De Silva et al. 2016; Jayawardena et al. 2016).

Endophytes are neutral or beneficial to their plant hosts (Backman and Sikora 2008). They boost host plant growth, fitness, stress tolerance, and alter interactions with pests and pathogens (Oono et al. 2015; Clay et al. 2016). Endophytes also provide protection against herbivory (O'Hanlon et al. 2012; Santangelo et al. 2015). More 
importantly, endophytes have potential as an unexplored source of candidate strains for potential biocontrol applications (Ek-Ramos et al. 2013; Oono et al. 2015). For example, endophytic Ampelomyces species parasitize powdery mildews (Busby et al. 2016). Since powdery mildews are biotrophs, their antagonists act mainly through antibiosis and mycoparasitism (Busby et al. 2016).

Biocontrol strategies utilize antagonistic mechanisms to disrupt the life cycle of pathogens (cf. Fig. 11), leading to the prevention of infection, reduction in colonization of host tissues, reductions in sporulation, and affecting the pathogen's ability to survive (Punja and Raj 2003; Busby et al. 2016). The hyperparasitic antagonism may be mediated by factors such as include the as production of lytic enzymes and/or antibiotics, while other biocontrol agents may induce the host plant's defense or just compete with the pathogen for nutrients and ecological niches (Yan et al. 2015; Busby et al. 2016; Lecomte et al. 2016; Schlegel et al. 2016).

Mejía et al. (2008) studied endophytes within the healthy leaves of Theobroma cacao, as well as their antagonism against the pathogenic Basidiomycota species Moniliophthora perniciosa (witches broom), Moniliophthora roreri (frosty pod rot) and the oomycete Phytophthora palmivora (black pod rot). The results showed that two endophytes, identfied as Colletotrichum gloeosporioides and Clonostachys rosea, respectively, decreased pod loss due to black pod rot, and reduced sporulating lesions in cacao pods caused by Moniliophthora roreri.

Endophytic fungi from various host plants have been shown to be effective biocontrol agents, including $\mathrm{Al}$ ternaria sp. and Cladosporium sp., isolated from wheat (Huang et al. 2016), and Alternaria alternata, isolated from grapevine leaves (Zhang et al. 2017b). The use of antagonistic endophytes as biocontrol agents, such as Trichoderma and Chaetomium, present an attractive option for management of certain plant diseases. It is important to screen potential endophytes through in-vitro experiments following field experiments under different environment conditions. In future research using molecular technologies (e.g., metagenomics), ecological dynamics are essential to developing commercial biocontrol agents, as these contribute to sustainable agriculture. It is critical to note that the species isolated as endophytes from a certain host plant may be pathogenic to other plants. Moreover, a careful risk assessment that excludes the possibility for the overproduction of mycotoxins is a mandatory prerequisite for registrations of new biocontrol agents, regardless of whether the producer organisms are endophytes.
Endophytes were isolated from healthy leaves and pods of Theobroma cacao plant

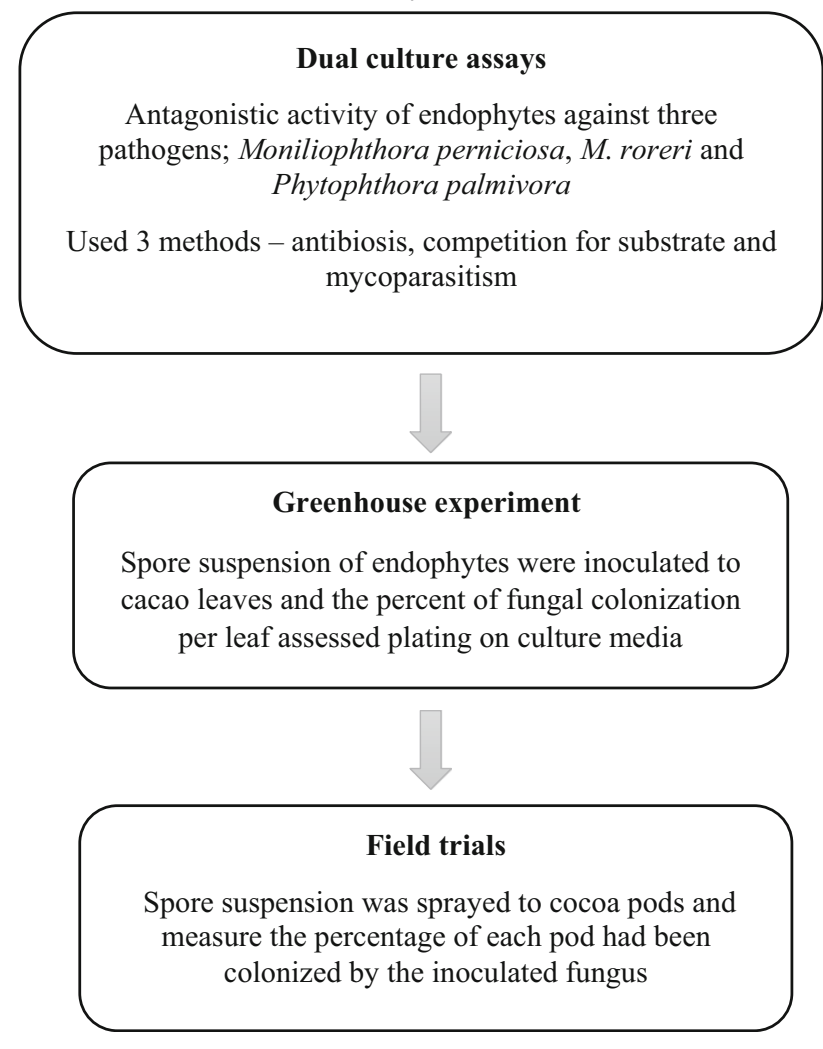

Fig. 11 Methodology used to assess biocontrol activity of endophytes against pathogens in cocoa plants

\section{Biocontrol of insects using fungi}

The loss in productivity due to crop damage from insects represents a serious threat to the agricultural sector. The global crop loss due to insects contributed to losses of almost $\$ 470$ billion each year (Culliney 2014), with the global expenditures on pesticides being in the range of $\$ 56$ billion in 2012. Strikingly, of the \$56 billion, only \$2-3 billion was spent on biopesticides (Marrone 2014). In the USA, insecticides contributed to almost $14 \%$ of all pesticide expenditures (Sabarwal et al. 2018).

The most common strategy for controlling insect invasions is the use of synthetic chemical pesticides, such as Chlorpyrifos, Acephate and Bifenthrin (Dai et al. 2019a). However, insecticidal resistance has become an undeniable phenomenon, and has led to the disastrous collapse of the pest control in many countries (Naqqash et al. 2016). There are also other concerns arising regarding the use of these synthetic chemicals, namely with food safety, adverse effects to non-target organisms-especially those 
beneficial antagonists of insects-and the environmental impact associated with the use of harmful chemical compounds (Sandhu et al. 2017). The drawbacks to conventional insecticides spurred the search for potent and ecofriendly biocontrol agents.

Biological control agents offer more advantages than their chemical counterparts, since they are safe for other non-target organisms and infect only specific species, with long-term results on target pests (Sanda and Sunusi 2016). In particular, the entomopathogenic fungi, have the capacity to reduce or eradicate insect populations. Most fungi used for the control of insect pests are ascomycetes, which are usually found in the soil and can cause natural outbreaks on their own when environmental conditions are favorable. Some fungal strains have been developed into commercial products because of their ability to be mass produced (e.g. Beauveria bassiana, Lecanicillium muscarium, Metarhizium anisopliae). These can infect a wide range of insect hosts. (cf. Fig. 12). Specific fungal strains in commercial products target insect groups such as Coleoptera, Diptera, Hemiptera, Hymenoptera, Lepidoptera and Orthoptera (Dauda et al. 2018).

Some entomopathogenic fungi can also exist as plant endophytes in a variety of hosts. They can exhibit dual functions, acting against insects and plant pathogens, thus giving protection to plant hosts. Moreover, they can have additional roles in endophytism, plant disease antagonism, growth promotion and rhizosphere colonization (Yun et al. 2017; Jaber and Ownley 2018).

Generally, the first mode of action of entomopathogenic fungi is to produce sticky spores to insure adhesion to the body of the host. The non-specific adhesion mechanism of the conidia is due to their hydrophobic properties, which has protein interactions with the hydrophobic exoskeleton of the susceptible host. The spores germinate quickly and initiate penetration of the insect exoskeleton. The fungal cells multiply in the hemocoel of the host's body, increasing the turgor pressure and eventually killing the insect. The entomopathogen grows in the host's cadaver to optimize spore production and dispersal under favorable environmental conditions (Roy et al. 2006). High numbers of spores are required to insure infection, with a minimum of $1 \times 10^{8}$ to $1 \times 10^{9}$ conidia/ml (Inglis et al. 2012).

Entomopathogenic fungi also produce secondary metabolites that can act as toxins with insecticidal effects. Proliferating protoplasts produce these compounds to weaken the host's defense mechanisms, causing rapid death (Hussain et al. 2014). The entomopathogens that produce toxins are more effective at killing the insect hosts as compared with those strains that do not produce such metabolites (Kershaw et al. 1999). There are a variety of active compounds produced by entomopathogenic fungi that exhibit insecticidal properties, as listed in Table 4 . Beauvericin (36 in Fig. 13) is a well-known active compound produced by entomopathogenic fungi. It plays a key
Fig. 12 Entomopathogens infecting insect hosts. a Acrodontium crateriforme, b Cordyceps militaris, c Ophiocordyceps nutans
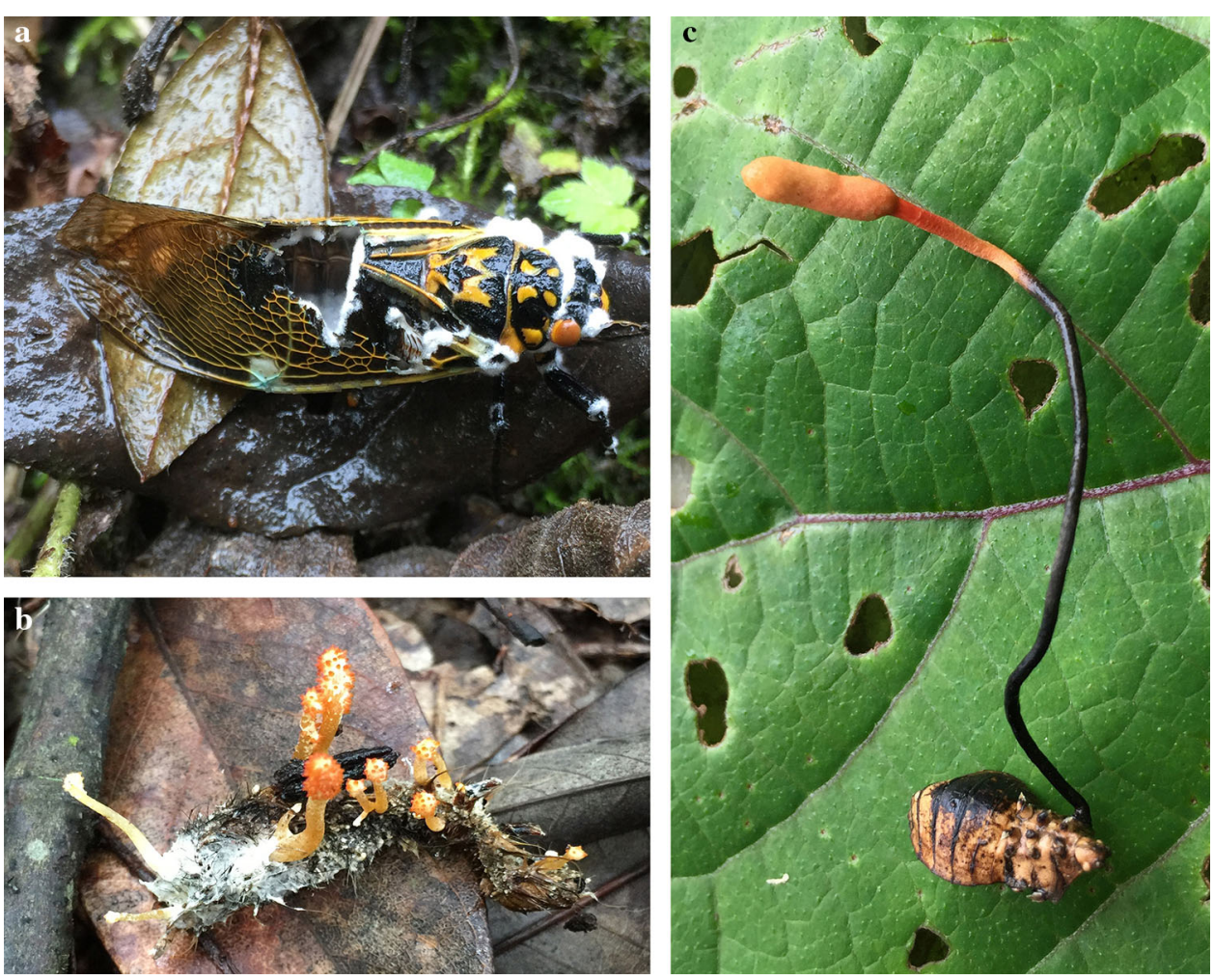
role in the virulence of fungi that infect arthropods (Rohlfs and Churchill 2011). It is a cyclic hexadepsipeptide, containing three D-hydroxyisovaleryl and three $\mathrm{N}$-methylphenylalanyl residues in alternating sequence, and belongs to the enniatin antibiotic family. It is structurally similar to enniatins; however, it differs in the nature of its N-methylamino acid (Wang and $\mathrm{Xu}$ 2012). It was first isolated from Beauveria bassiana (Hamill et al. 1969) and later from Fusarium species (Liuzzi et al. 2017). Beauvericin has a strong insecticidal function against a broad spectrum of insects. Since the target insects are moving organisms, the entomopathogenic fungi producing beauvericin are more effective insecticidal agents than the direct use of the compound. Beauvericin was first discovered to have insecticidal activity by Hamill et al. (1969). Other studies proved the efficacy of beauvericin in killing other insects, such as Calliphora erythrocephala, Lygus spp. (Leland et al. 2005), Aedes aegyptii (Grove and Pople 1980), Spodoptera frugiperda and Schizaphis graminum (Ganassi et al. 2002); however, it can also be toxic to bees, thus posing a threat to other beneficial insects when applied in the field.

Bassianolide (37) a cyclic depsipeptide from Lecanicilium lecanii, exhibits moderate cytotoxicity and an immunosuppressive effect to insect hosts. It can cause significant maximum mortality to Plutella xylostella at $0.5 \mathrm{mg} / \mathrm{ml}$ concentrations (Keppanan et al. 2018). As in other groups of fungal cyclopeptides, several variants if bassianolies are known (Matsuda et al. 2004).

Destruxins (38-40), the cyclic hexadepsipeptide mycotoxins are produced by Metarhizium anisopliae. They can kill a variety of insect pests. The purified destruxins can cause toxic effects on the larval developmental stage of mosquitoes (Aedes aegyptii) with high mortality rates (Ravindran et al. 2016). The study of Dong et al. (2016) also revealed positive correlations between destruxin production and blastospore formation, and the producer strain has the potential to be developed into a mycoinsecticide.

Enniatins (41-44) are only produced by Fusarium species. They act as ionophores that bind with ammonium in the transport of ions in the lipid bilayer membrane of the cell. This ionophoric property of enniatins leads to the toxic action in the cell through the disturbance of the normal physiological concentration. Their best studied derivative, Enniatin B was previously shown to exhibit insecticidal activity against blowfly (Calliphora erythrocephala) and mosquito larvae (Aedes aegypti) (Grove and Pople 1980).

The successful application of entomopathogenic fungal strains relies on several factors, such as level of virulence, production efficiency and level of safety to humans and other non-target species. Virulence depends on a complex of factors, such as spore hydrophobicity, which is involved in the conidial adhesion, germination polarity of the spores wherein unidirectional spores are more virulent than multi-

Table 4 Some insecticidal compounds produced by entomopathogenic fungi

\begin{tabular}{|c|c|c|}
\hline Entomopathogen & Insecticidal compounds & References \\
\hline Beauveria bassiana & $\begin{array}{l}\text { Bassiacridin } \\
\text { Bassianin } \\
\text { Bassianolide } \\
\text { Beauvericins (36) } \\
\text { Beauverolides }\end{array}$ & $\begin{array}{l}\text { Matsuda et al. (2004), Quesada-Moraga et al. } \\
\text { (2004), Ohshiro et al. (2006), Heneghan et al. } \\
\text { (2011), Fisch et al. (2011), Süssmuth et al. (2011) }\end{array}$ \\
\hline Beauveria tenella & $\begin{array}{l}\text { Beauverolides, } \\
\text { Beauvericins (36) } \\
\text { Bassianolide (37) }\end{array}$ & $\begin{array}{l}\text { Ohshiro et al. (2006), Namatame et al. (1999), } \\
\text { Süssmuth et al. (2011) }\end{array}$ \\
\hline Lecanicillium lecanii & Helvolic acid & Kildgaard et al. (2014), Liang et al. (2016) \\
\hline $\begin{array}{l}\text { Fusarium spp.;"Paecilomyces" (Isaria) } \\
\text { fumosoroseus, Paecilomyces (Isaria) } \\
\text { tenuipes }\end{array}$ & Beauvericins (36) & \\
\hline Isaria sp. & Beauverolides & $\begin{array}{l}\text { Briggs and Atkins (1966), Isogai et al. (1978), } \\
\text { Elsworth and Grove (1980), Grove (1980) }\end{array}$ \\
\hline Metarhizium anisopliae & Destruxins (38-40) & Wang et al. (2012), Yoshida et al. (2014) \\
\hline
\end{tabular}


Fig. 13 Chemical structures of insecticidal compounds from fungi

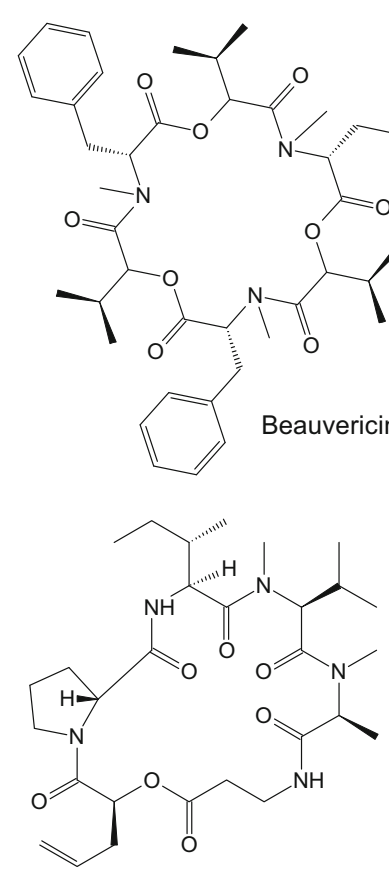

Destruxin A (39)

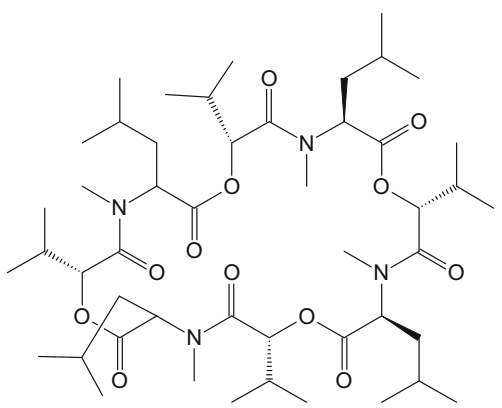

Bassianolide (37)<smiles>[R]C[C@H](OC(=O)CCNC(=O)C(C)N(C)C(=O)[C@@H](C(C)C)N(C)C(=O)[C@H](NC(=O)[C@@H]1CCCN1C(=O)[C@H](C)CC)[C@H](C)CC)C(=O)N(C)C</smiles>

Destruxin $\mathrm{B}, \mathrm{R}=\mathrm{CH}-\left(\mathrm{CH}_{3}\right)_{2}(\mathbf{3 8})$ Destruxin $E, R=\prod_{O}(\mathbf{4 0})$

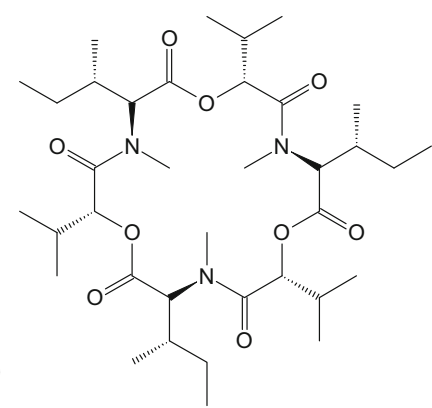

Enniatin A (41)

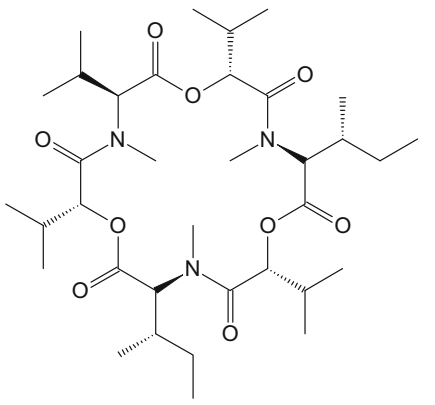

Enniatin A1 (42)<smiles>[R]C(C)[C@H](C(=O)O[C@H](C(=O)N(C)[C@H](C(=O)O[C@H](C(=O)N(C)[C@@H](C(=O)O[C@H](C(=O)N(C)C(C(C)C)C(C)C)C(C)C)C(C)C)C(C)C)C(C)C)C(C)C)N(C)C</smiles>

Enniatin $\mathrm{B}, \mathrm{R}=\mathrm{CH}_{3}(\mathbf{4 3})$

Enniatin $\mathrm{B} 1, \mathrm{R}=-\mathrm{CH}_{2} \mathrm{CH}_{3}$ (44) directional ones, presence of hydrolytic enzymes to breach the host's defense wall, and sensitivity to abiotic factors like temperature and humidity. Moreover, entomopathogenic fungi should only be considered for commercialization if they demonstrate high production efficiency, wherein the strains have minimum requirements for growth and can be grown and mass produced in solid substrate (Hussain et al. 2014), thus reducing production costs.

Certain techniques should be considered in order to increase the effectiveness of mycoinsecticides. Since insect larvae usually embed themselves into plant tissues or soil and do not feed on crops (Hall 1998), they do not typically stay in a specific location and therefore are very difficult to target. Spray application can be inefficient at this stage and depositing mycoinsectides over a field or crop may not be effective. Therefore, granular formulations which coat dry spores onto bran or grains, or the drying and fragmentation of mycelium to hold spores in starch, can both be effective methods to treat insects in the field (Hall 1998). Adding the granular substances to mycoinsecticides increases the accuracy and efficiency of the spray.

Several tactics have been identified by Lacey and Kaya (2007) to increase the effectiveness of mycoinsecticides. Preventive applications could be inefficient because the residues are not long-lasting, and therefore they should be applied only when the target pest is seen. Mycoinsecticides should be applied before the pest population reaches peak numbers, and therefore early application is essential. Timing is also important: identifying the life cycle of the host, which has a higher probability of being in contact with the spores, could also increase the effectiveness of the mycoinsecticide. For example, Beauveria bassiana is more efficient in infecting active nymphs than winged adults. Moreover, mycoinsecticides should not be applied during 
droughts because the environmental conditions are not favorable for the germination of the spores.

Approximately 750 fungal species have been identified as insect pathogens; however, in 1998 only 12 species were utilized as mycoinsecticides in insect sprays (Wraight and Carruthers 1998). By 2007 the number of mycoinsecticides had increased to about 110 commercially available products in the market (de Faria and Wraight 2007). The number has continued to increase, with almost 2700 arthropod pesticides introduced worldwide (Cock et al. 2010), in which about 230 species of biological control agents have been marketed and available for commercial use (van Lenteren 2012). The demand and commercial application of microbial pesticides have increased tremendously. Of 82 microbial biopesticides registered in Brazil, nearly $46 \%$ are mycoinsecticides (Mascarin et al. 2018). Fungal biopesticides contributed to a large portion of this increased demand and popularity in pest management in the past two decades (Jaronski and Mascarin 2017). Several products have already disappeared from the international marketplace because they were not commerically successful, but a substantial number of fungal species have been exploited (Kabaluk et al. 2010; Lacey et al. 2015) (Fig. 12).

De Faria and Wraight (2007) and Mascarin et al. (2018) listed representatives of mycoinsecticides derived from Beauveria, Isaria, Lecanicillium, and Metarhizium species found in the market. Most developed mycoinsecticdes were made for European and American markets, whereas only a few were aimed at the African and Asian markets (Table 5).

There have been continual obstacles and challenges to the development and commercialization of fungal biological control agents for use in controlling insect populations, ranging from gaps in understanding the basic biological knowledge to their potential socio-economic impact. Problems regarding the effectiveness of insecticides are linked to economic production. The increase in pest-resistance to chemical formulas has led to drastic environmental problems. New chemical insecticides are not being developed quickly enough, and therefore there is room for microbial insecticides, which are growing at the rapid pace of 10-25\% per year (Starnes et al. 1993), within the market. Enhancing the virulence of potential fungal species is essential for mycoinsecticides to develop and reach their market potential.

The future of mycoinsecticides is promising, with continued research to increase pathogenic virulence of entomopathogenic fungi in order to achieve commercial success with genetic and physiological engineering. Further studies should be conducted to determine the fungal traits responsible for the effectiveness of mycoinsecticides, enhancement of their virulence, and development of ecofriendly and effective pest management strategies.

\section{Biocontrol of nematodes and fungal nematicides}

Plant-parasitic nematodes are parasites which cause severe damage to many economically important crops such as tomatoes, potatoes and wheat. For example, around US \$80 billion of yield losses are caused annually by damage from plant root-knot nematodes, such as $M$. javanica and $M$. incognita (Li et al. 2007). Nematicidal chemicals that were once rather effective, such as methyl bromide, have ultimately been banned because they are broad-spectrum biocides that kill all life in the soil and contribute to the depletion of the ozone layer, thereby causing grave problems to the environment. Therefore, in recent years there have been great efforts in both academia and industry to find ecologically viable alternatives.

Nematophagous fungi are capable of controlling plant parasitic nematodes through antagonistic behaviour (Zaki and Siddiqui 1996). There are more than 700 species of nematophagous fungi, which are found in fungal taxa including Mucoromycota, Basidiomycota, Ascomycota and Chytridiomycota (Li et al. 2005; Degenkolb and Vilcinskas 2016). These nematophagous fungi are categorized into four groups according to their mode of parasitism: nematode-trapping (otherwise known as predacious); nematode egg and female parasites; endoparasitic; and toxin-producing fungi (Li et al. 2005; Degenkolb and Vilcinskas 2016). Nematode-trapping fungi form specific trapping structures on hypha, such as adhesive knobs, constricting rings and adhesive networks; these three trapping devices can be categorized further into seven types: simple adhesive branches, unstalked adhesive knobs, stalked adhesive knobs, non-constricting rings, constricting rings, two dimensional networks and three-dimensional networks (Rubner 1996).

The constricting ring trap is the most sophisticated morphological adaptation, which can be found in fungi that are no accommodated in the genus Drechslerella (Baral et al. 2018). When a nematode enters the ring, the three cells swell, forming three sphaerical structures that trap and immobilize the nematode (Jansson and Lopez-Llorca 2004). Endoparasitic fungi attack nematodes orally or by penetration of spores or zoospores through the cuticles of the nematode host (Moosavi et al. 2011). After infection, the hyphae develop inside the nematode and digests its content. For example, the spores of Catenaria anguillulae are ingested by sedentary nematodes such as Heterodera spp. The spores germinate in the esophagus and the mycelia then digest the nematodes; 10-12 $\mathrm{h}$ after the infection, zoosporangia develop to release new zoospores 
Table 5 Representative mycoinsecticides in the market (de Faria and Wraight 2007; Mascarin et al. 2018)

\begin{tabular}{|c|c|c|c|c|}
\hline Fungal species & $\begin{array}{l}\text { Marketed } \\
\text { trade name }\end{array}$ & Country registered & Pests targeted & Manufacturer \\
\hline \multirow[t]{15}{*}{$\begin{array}{c}\text { Metarhizium } \\
\text { anisopliae }\end{array}$} & Arizium & Brazil & $\begin{array}{l}\text { Mahanarva fimbriolata, Notozulia (Zulia) } \\
\text { entreriana }\end{array}$ & TecniControl \\
\hline & $\begin{array}{l}\text { BioMetha GR } \\
\text { Plus }\end{array}$ & Brazil & M. fimbriolata, Notozulia sp., Deois sp. & Biotech \\
\hline & Bioninsect & Brazil & M. fimbriolata, N. entreriana, D. flavopicta & Koppert do Brasil \\
\hline & GR-INN & Brazil & M. fimbriolata, N. entreriana, D. flavopicta & Agrivalle \\
\hline & $\begin{array}{l}\text { Metamax } \\
\text { Líquido }\end{array}$ & Brazil & M. fimbriolata & Bio Soja \\
\hline & Metapremium & Brazil & M. fimbriolata, N. entreriana, D. flavopicta & Biopremium \\
\hline & Metarfito & Brazil & M. fimbriolata, N. entreriana & $\begin{array}{l}\text { Fitoagro Controle } \\
\text { Biológico }\end{array}$ \\
\hline & $\begin{array}{l}\text { Metarhizium } \\
\text { JCO WP }\end{array}$ & Brazil & M. fimbriolata, N. entreriana, D. flavopicta & $\mathrm{JCO}$ \\
\hline & $\begin{array}{l}\text { Metarhizium } \\
\text { Oligos }\end{array}$ & Brazil & M. fimbriolata, N. entreriana, D. flavopicta & Oligos Biotecnologia \\
\hline & $\begin{array}{l}\text { Metarhizium } \\
\text { Probio }\end{array}$ & Brazil & M. fimbriolata, N. entreriana, D. flavopicta & Probio \\
\hline & MethaControl & Brazil & M. fimbriolata, N. entreriana, D. flavopicta & Simbiose \\
\hline & Methamax EC & Brazil & M. fimbriolata & Novozymes \\
\hline & Metie & Brazil & N. entreriana & Ballagro \\
\hline & Opala & Brazil & M. fimbriolata, $N$. entreriana & $\begin{array}{l}\text { Lab. Biocontrole } \\
\text { Farroupilha }\end{array}$ \\
\hline & $\begin{array}{l}\text { Real } \\
\text { Metarhizium }\end{array}$ & $\begin{array}{l}\text { Kenya, South Africa, } \\
\text { Ethiopia, Ghana }\end{array}$ & Mealybugs, thrips, whiteflies & Real IPM (Kenya) \\
\hline \multirow[t]{3}{*}{$\begin{array}{l}\text { Metarhizium } \\
\text { brunneum }\end{array}$} & Met52 EC & USA & Whiteflies, thrips, weevils & $\begin{array}{l}\text { Novozymes Biologicals } \\
\text { (USA) }\end{array}$ \\
\hline & $\begin{array}{l}\text { Met52 } \\
\text { Granular }\end{array}$ & USA, Europe & Weevils, thrips & $\begin{array}{l}\text { Novozymes Biologicals } \\
\text { (USA) }\end{array}$ \\
\hline & Bio1020 & Netherlands & Weevils & $\begin{array}{l}\text { Novozymes Biologicals } \\
\text { France }\end{array}$ \\
\hline \multirow{13}{*}{$\begin{array}{l}\text { Beauveria } \\
\text { bassiana }\end{array}$} & Adral & Colombia & Aphids & Bio-Crop (Colombia) \\
\hline & $\begin{array}{l}\text { Agrivalle } \\
\text { AUIN }\end{array}$ & Brazil & $\begin{array}{l}\text { Bemisia tabaci, Cosmopolites sordidus, Dalbulus } \\
\text { maidis, Tetranychus urticae }\end{array}$ & Agrivalle \\
\hline & Ballvéria & Brazil & B. tabaci & Ballagro \\
\hline & Bassicore SC & Colombia & Whiteflies & $\begin{array}{l}\text { Core Biotechnology } \\
\text { (Colombia) }\end{array}$ \\
\hline & BeauveControl & Brazil & B. tabaci, D. maidis, T. urticae & Simbiose \\
\hline & $\begin{array}{l}\text { Beauveria } \\
\text { JCO }\end{array}$ & Brazil & B. tabaci, C. sordidus, D. maidis, T. urticae & $\mathrm{JCO}$ \\
\hline & BioExpert & Colombia & Whiteflies, thrips & $\begin{array}{r}\text { Live Systems } \\
\text { Technology } \\
\text { (Colombia) }\end{array}$ \\
\hline & Bioveria WP & Brazil & B. tabaci, C. sordidus, D. maidis, T. urticae & Bioenergia do Brasil SA \\
\hline & Bovemax EC & Brazil & $\begin{array}{l}\text { H. hampei, Diaphorina citri, Hedypathes } \\
\text { betulinus }\end{array}$ & Novozymes \\
\hline & Boveril WP & Brazil & $\begin{array}{l}\text { B. tabaci, Hypothenemus hampei, Gonipterus } \\
\text { scutellatus, T. urticae }\end{array}$ & Koppert do Brasil \\
\hline & Broadband & South Africa & Whiteflies, thrips & BASF South Africa \\
\hline & Cadete SC & Colombia & Whiteflies, thrips & $\begin{array}{l}\text { Mycros Internacional } \\
\text { (Colombia) }\end{array}$ \\
\hline & Ecobass & Brazil & B. tabaci, C. sordidus, D. maidis, T. urticae & Toyobo do Brasil \\
\hline
\end{tabular}


Table 5 continued

\begin{tabular}{|c|c|c|c|c|}
\hline Fungal species & $\begin{array}{l}\text { Marketed } \\
\text { trade name }\end{array}$ & Country registered & Pests targeted & Manufacturer \\
\hline & Granada & Brazil & B. tabaci, C. sordidus, D. maidis, T. urticae & $\begin{array}{l}\text { Lab. Biocontrole } \\
\text { Farroupilha }\end{array}$ \\
\hline $\begin{array}{l}\text { Isaria } \\
\text { fumosorosea }\end{array}$ & Challenger & Brazil & Diaphorina citri & Koppert do Brasil \\
\hline \multirow[t]{2}{*}{ Isaria javanica } & $\begin{array}{l}\text { PFR-97 20\% } \\
\text { WDG }\end{array}$ & USA, Mexico & Whiteflies, aphids, thrips, mealybugs, & Certis, Inc. (USA) \\
\hline & PreFeRal WG & Europe & Whiteflies & Biobest (Belgium) \\
\hline \multirow[t]{3}{*}{ Isaria spp. } & Nofly & USA & Whiteflies, aphids, thrips, psyllids, & $\begin{array}{l}\text { Novozymes Biologicals } \\
\text { (USA) }\end{array}$ \\
\hline & Pae-Sin & Mexico & Whiteflies & $\begin{array}{l}\text { Agrobiológicos del } \\
\text { Noroeste - }\end{array}$ \\
\hline & Successor SC & Colombia & Whiteflies, aphids, thrips & $\begin{array}{c}\text { Live Systems } \\
\text { Technology } \\
\text { (Colombia) }\end{array}$ \\
\hline $\begin{array}{l}\text { Lecanicillium } \\
\text { muscarium }\end{array}$ & Mycotal & Europe, Japan & Whiteflies, thrips & $\begin{array}{l}\text { Koppert Biological } \\
\text { Systems }\end{array}$ \\
\hline \multirow[t]{3}{*}{$\begin{array}{l}\text { Lecanicillium } \\
\text { spp. }\end{array}$} & Verzam & $\begin{array}{l}\text { Honduras, El Salvador, } \\
\text { Nicaragua, Jamaica }\end{array}$ & Whiteflies, aphids, thrips & $\begin{array}{l}\text { Escuela Agrícola } \\
\text { Panamericana }\end{array}$ \\
\hline & Vercani WP & Colombia & Whiteflies & $\begin{array}{l}\text { Natural Control } \\
\text { (Colombia) }\end{array}$ \\
\hline & Lecafol & Uruguay & Whiteflies & $\begin{array}{l}\text { Lage y Cía. S.A., } \\
\text { Uruguay }\end{array}$ \\
\hline
\end{tabular}

(Mankau 1980). The motile zoospores are adapted by having positive tropisms toward nematodes (Kumar et al. 2017). Nematode egg parasitic fungi play a key role in infecting the eggs of nematodes on two levels. Some fungi directly infect the nematode eggs by penetrating the eggshell, while others indirectly affect the content of eggs, such as larvae or embryos (Jansson and Lopez-Llorca 2004). Recent studies found that Ijuhya vitellina forms hyphae from the infected egg shells of the cereal cyst nematode Heterodera filipjevi to develop into microsclerotia (Ashrafi et al. 2017a). Monocillium gamsii and $M$. bulbillosum are two nematode-associated fungi parasitic to eggs of H. filipjevi (Ashrafi et al. 2017b). The first record of dark septate endophytes with nematicidal effects was reported from the new genus Polyphilus, represented by two new species (Ashrafi et al. 2018). Actually Polyphilus spp. have been isolated from nematode eggs as well as from healthy plant material.

Several studies related to nematode parasitic fungi that are specific to economically important crops have been conducted (Table 7). Cochliobolus sativus (on wheat and barley), Dendriphiopsis spp. (on tomato plants) and Drechmeria coniospora are some controlling agents for root knot disease caused by nematodes on wide variety of crops, especially tomatoes (Jansson et al. 1985). A combination of Trichoderma species and nematode-trapping fungi was most effective in controlling plant-parasitic nematodes through egg parasitism (Szabo 2014). Moreover, there are some fungi which are capable of controlling nematode diseases in animals (Zhang et al. 2007). For example, animals infected by plant-parasitic nematodes are fed with fungal mycelium containing chlamydospores of nematode trapping fungi, e.g. Duddingtonia flagrans (Zhang et al. 2007). When fed to the animals. these spores produce traps in the faeces and surrounding grasses to capture newly hatched nematode juveniles (Zhang et al. 2007). Purpureocillium lilacinum is capable of controlling root-knot nematodes such as $M$. javanica and $M$. incognita on tomato, eggplant and other vegetable crops (Moosavi 2014). Some studies have reported the antagonistic behaviour of Arthrobotrys dactyloides against root knot nematodes on tomato plants, but other experiments with Metacordyceps chlamydosporia did not significantly reduce nematode population (Nordbring-Hertz et al. 2011). Cochliobolus sativus was also reported to be an effective biological control agent in controlling nematodes in Botswana (Mubyana-John and Wright 2011). Table 6 is a synopsis of nematode parasitic fungi, and the unique trapping mechanisms of nematode-trapping fungi. The combination of Purpureocillium lilacinum (previously referred to as "Paecilomyces lilacinus") and Dactylella lysipaga was experimentally proved to be antagonistic to 
Table 6 Taxonomy of nematode parasitic fungi, their trapping mechanisms and parasitic nematodes.. Source Zhang et al. (2014a, b)

\begin{tabular}{|c|c|c|c|}
\hline Nematophagous fungal group & Phyla & Fungal name & Trapping structures \\
\hline \multirow[t]{7}{*}{ Nematode-trapping } & \multirow[t]{2}{*}{ Zoophagomycota } & Stylopage & Adhesive hyphae \\
\hline & & Cystopage & Adhesive hyphae \\
\hline & \multirow[t]{4}{*}{ Ascomycota } & Arthrobotrys/Orbilia & Adhesive networks \\
\hline & & Dactylellina/Orbilia & \\
\hline & & Drechslerella/Orbilia & Adhesive knobs and/or non constricting rings \\
\hline & & Gamsylella/Orbilia & Constricting rings \\
\hline & Basidiomycota & Hohenbuehelia & $\begin{array}{l}\text { Adhesive branches or unstalked knobs } \\
\text { Adhesive "hour-glass" knobs }\end{array}$ \\
\hline \multirow[t]{7}{*}{ Endoparasitic } & Chytridiomycota & Myzocytiopsis & Zoospores \\
\hline & Blastocladiomycota & $\begin{array}{l}\text { Haptoglossa } \\
\text { Catenaria }\end{array}$ & "Gun cells", injection \\
\hline & \multirow[t]{4}{*}{ Ascomycota } & Harposporium/Podocrella & Zoospores \\
\hline & & Meria & Ingested conidia \\
\hline & & Haptocillium/Cordyceps & Adhesive conidia \\
\hline & & Hirsutella & Adhesive conidia \\
\hline & Basidiomycota & Nematoctonus/Hohenbuehelia & Adhesive "hour-glass" knobs \\
\hline \multirow[t]{2}{*}{ Toxin-producing } & \multirow[t]{2}{*}{ Basidiomycota } & Coprinus & Toxic droplets \\
\hline & & $\begin{array}{l}\text { Pleurotus } \\
\text { (Omphalotus) }\end{array}$ & Toxin, spiny structures \\
\hline
\end{tabular}

the root-knot nematode Meloidogyne javanica as well as the cereal cyst nematodes Heterodera avenae and Radopholus similis on tomato barley and banana plants (Zhang et al. 2014a, b). In another study, the combination of Purpureocillium lilacinum and Monacrosporium lysipagum was shown to be antagonistic to Meloidogyne javanica, the cereal cyst nematode (Zhang et al. 2014a, b).

Few studies conducted thus far have proven the efficiacy of nematode parasitic fungi; however, individually these fungi do not possess all the desirable characters required to serve as high potential nematode control agents. Thus combinations of nematophagous fungi can be more effective, as these combinations are capable of being parasitic in all stages of the nematode life cycle. Therefore, it is necessary to develop new techniques to facilitate research on nematode parasitic fungi, especially for tracing high potential strains in the environment. More studies are needed to improve and introduce more efficient strategies to produce biological control agents without harming nontargeted nematode species.

Liquid fermentation and solid fermentation are two methods of mass producing nematophagous fungi. Solid culturing is preferable for fungi that cannot produce spores in liquids (Zhang et al. 2014a, b). However, liquid culturing is widely used for the mass production of the spores and mycelium of targeted fungi (Zhang et al. 2014a, b). In fact, the combination of these two methods enhances the effectiveness of the mass production of fungi, as the liquid method can be used for producing mycelia and the solid method for producing conidia. The formulation of the fungal production is important in its commercialization as a bio control agent. Formulations are powders, wettable powders, emulsions, oil solutions, granular formulations, blending agents and microcapsules (Liu and $\mathrm{Li}$ 2004). Microencapsulation is a new method of commercializing bio control fungi (Patel et al. 2011). The latter researchers developed a novel capsule system using Hirsutella rhossiliensis. Jin and Custis (2011) also introduced a modified method for microencapsulation of Trichoderma conidia using sugar, which was then developed as a sprayable formulation.

Toxin-producing fungi secrete nematicidal metabolites to attack and immobilise nematodes before the development of hyphae inside the nematode (Degenkolb and Vilcinskas 2016). For example, Purpureocillium lilacinum produces acetic acid to immobilize juvenile parasitic nematodes (Djian et al. 1991). According to previous studies, more than 270 toxin-producing fungi have been recorded, including 230 nematicidal toxic compounds (Zhang et al. 2011a; Li et al. 2007). Recent examples include the report on the new genus and species Pseudobambusicola thailandica, which can control nematodes using its secondary metabolites, such as monocerin and deoxyphomalone (Rupcic et al. 2018). Linoleic acid is one of the nematicidal compounds that can be isolated from many fungi, including Arthrobotrys species, in which the production of this compound increases with the number of traps (Anke et al. 1995). Pleurotus pulmonarius and 
Hericium coralloides are two basidiomycetes that exhibit strong nematicidal effect against Caenorhabditis elegans. p-Anisaldehyde (46) and other aromatic metabolites, as well as fatty acids, were identified as active principles (Stadler et al. 1994) (Fig. 14).

Metabolites with moderate nematicidal activity have been recently reported from a Sanghuangporus sp. collected in Kenya. In addition to 3, 14'-bihispidinyl and hispidin and the new dcerivative phelligridin L (49), with moderate effects on Caenorhabditis elegans (Chepkirui et al. 2018). Ophiotine (45), a depsipeptide from a species of the Phaeosphariaceae isolated from nemtatode eggs, was reported to have a moderate nematicidal effect on Heterodera filipjevi (Helaly et al. 2018a). Chaetomium globosum produces flavipin, which inhibits egg hatching and juvenile mobility of the root-knot nematode (Meloidogyne incognita) and the hatching of soybean cysts (Heterodera glycines) (Nitao et al. 2002). Chaetoglobosin A and its derivate 19- $O$-acetylchaetoglobosin $\mathrm{A}$ are two other recently demonstrated chemical compounds isolated from Ijuhya vitellina, a parasitite of the eggs of Heterodera filipjevi (Ashrafi et al. 2017a, b). However, the most important fungal nematicides found to date are (a) PF-1022A, a precursor of the semisynthetic drug emodepside (48), which is used in veterinary medicine (Jeschke et al. 2005); and (b) the cyclopeptide omphalotin A (47) from cultures of the basidiomycete genus Omphalotus, which was also developed as a nematicide against Meloidogyne, but did not yet make it to the market because of unfavourable costs of goods (Sandargo et al. 2019a).

Many studies have shown the parasitic efficiency of nematode parasitic fungi, but it is necessary to develop IPM strategies to optimize the ability nematophagous fungi to colonize plant roots. High virulence strains and
Fig. 14 Chemical structures of fungal metabolites with nematicidal activity

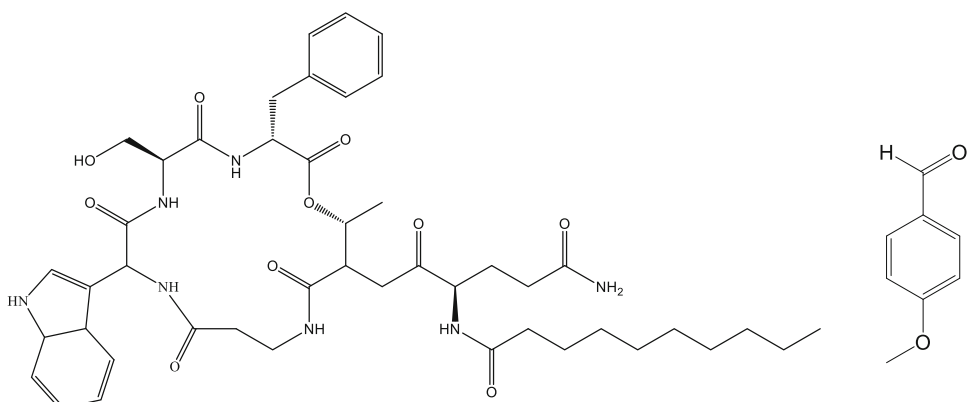

Ophiotine (45)

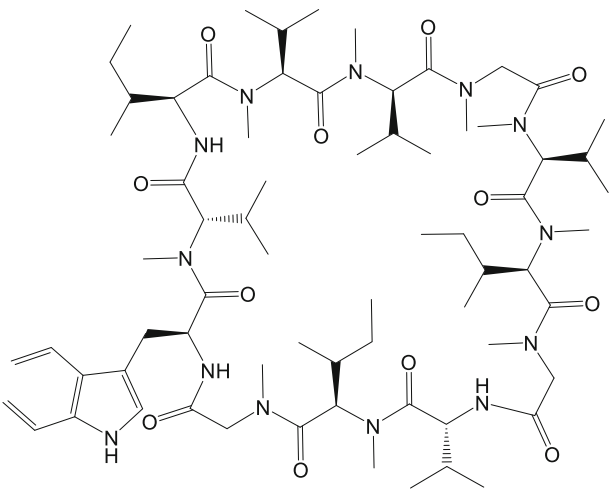<smiles></smiles>

Omphalotin A (47)

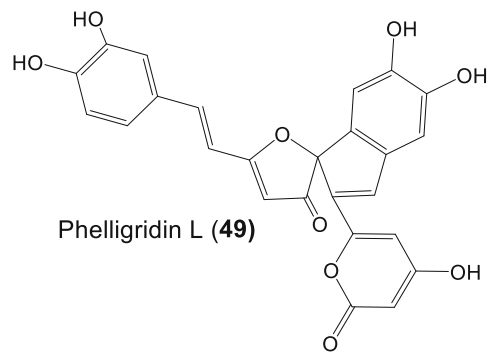


Fig. 15 Mechanism of actions implemented by antagonistic fungal species for management of rust diseased plants

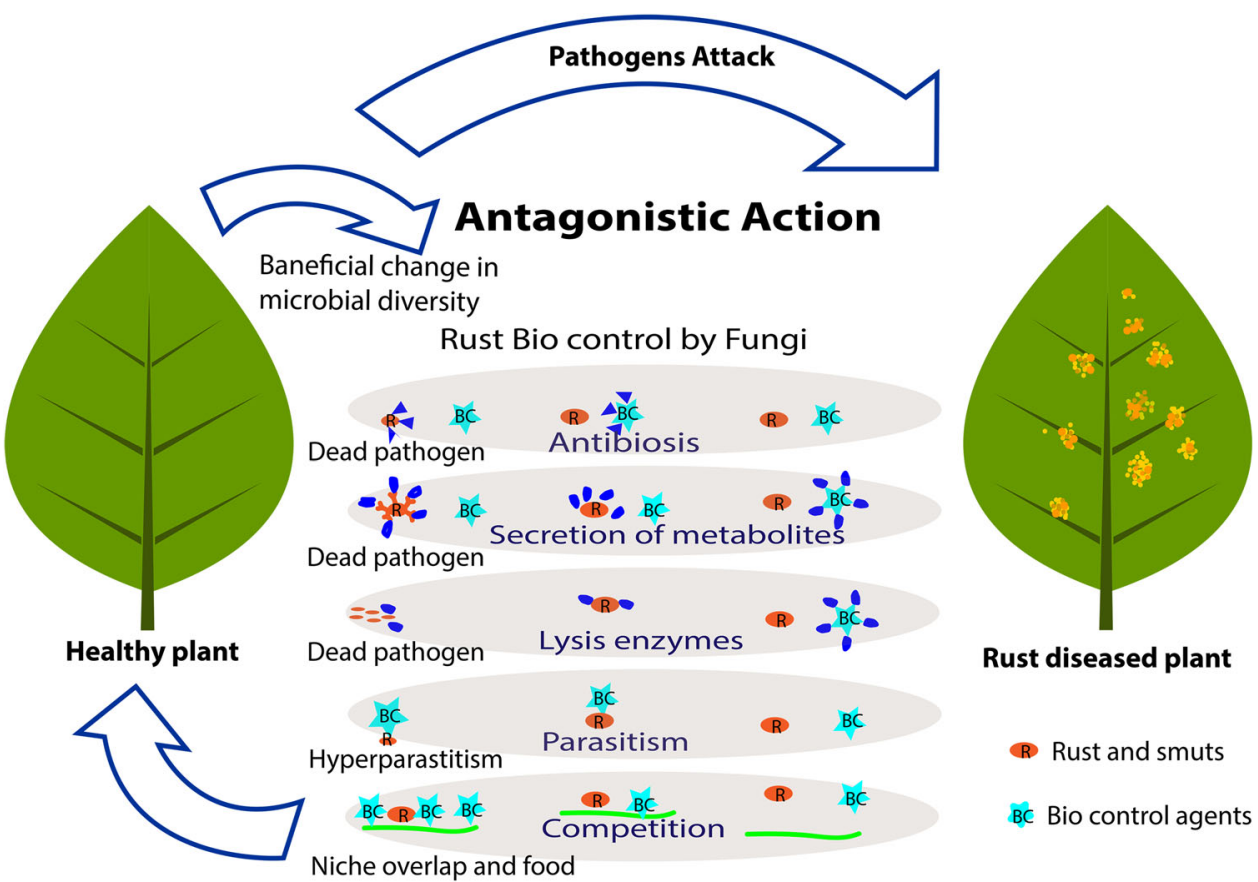

Table 7 Commercial biological nematicides based on nematophagous fungi.. Source Zhang et al. (2014a, b), updated by using the current taxonomy

\begin{tabular}{|c|c|c|c|c|}
\hline Products & Fungi & Modes of action & Nematode targets & References \\
\hline Bicon & $\begin{array}{l}\text { Purpureocillium } \\
\text { lilacinum }\end{array}$ & $\begin{array}{l}\text { Egg and female } \\
\text { parasitism }\end{array}$ & Unspecified & Poinar and Georgis (1994) \\
\hline Miexianning & $\begin{array}{l}\text { Purpureocillium } \\
\text { lilacinum }\end{array}$ & $\begin{array}{l}\text { Egg and female } \\
\text { parasitism }\end{array}$ & $\begin{array}{l}\text { Root-knot nematodes parasitizing } \\
\text { tobacco }\end{array}$ & Shuhua et al. (2002), Zhou and Mo (2002) \\
\hline Nemout & Unspecified $\mathrm{NTF}^{\mathrm{a}}$ & Trapping & Meloidogyne javanica & $\begin{array}{l}\text { Al-Hazmi et al. (1982), Ibrahim (1994), } \\
\text { Williams et al. (1999) }\end{array}$ \\
\hline Paecil/Bioact & $\begin{array}{l}\text { Purpureocillium } \\
\text { lilacinum }\end{array}$ & $\begin{array}{l}\text { Egg and female } \\
\text { parasitism }\end{array}$ & Unspecified & Holland et al. (1999) \\
\hline Royal 350 & $\begin{array}{l}\text { Arthrobotrys } \\
\text { irregularis }\end{array}$ & Trapping & Meloidogyne spp. & Cayrol et al. (1978) \\
\hline Xianchongbike & $\begin{array}{l}\text { Metacordyceps } \\
\text { chlamydosporia }\end{array}$ & $\begin{array}{l}\text { Egg and female } \\
\text { parasitism }\end{array}$ & $\begin{array}{l}\text { Root-knot nematodes } \\
\text { Parasitizing tobacco, peanut, } \\
\text { soybean and watermelon }\end{array}$ & Zhou and Mo (2002) \\
\hline
\end{tabular}

formulations are important for the development of more efficient commercial products. The utilization of the secondary metabolites of fungi as nematicides is not satisfactory at the commercial level as there are few bionematicides available in the market. On the other hand, integrated nematode management strategies can be implemented to improve the effectiveness of nematicidal action and minimize the use of chemical nematicides to the soil. Future bio products should be targeted not only for one species of nematode, but to disease complexes in concert with other pathogens like fungi, bacteria and viruses.

\section{Biocontrol of weeds and herbicides from fungi}

Weeds are plants that grow in a location where their presence has become undesirable due to their adverse effects on the ecosystem or human activities (Norris 1992; Kent 1994; Gadermaier et al. 2014). This is mainly due to 
the fact that when a plant is introduced to a new environment without the influence of their natural enemies they can grow without any obstruction and harm the native population by taking the form of a weed (Kendrick 1992). Thus, weeds can cause problems not only to the crop yield and quality, but also to the nature by invading native environment and its species composition. In order to ensure the balance of ecosystems, global crop production and food security; weed management is an important factor to be considered (Bjawa 2014).

During the early 1990s weed management was dominated by mechanical methods (Sommer 1996). The importance of mechanical weed control has become limited as it causes soil erosion and nutrient losses (Zimdahl 1993). Thus, chemical herbicides became the viable alternative. However, due to continuous use of the same herbicide with the same mode of action, herbicide resistance increased and for the last 20 years no chemical has been manufactured with a different mode of action than the previous products (Duke 2012). Researchers also identified the herbicidal toxic impact on non-target soil organisms which play an important role in degrading and decomposing organic matter (Subhani et al. 2015; Zain et al. 2013). Therefore, the need for biological weed control as well as development of bio-herbicides has become important.

Biological control of weeds (see the diagram in Fig. 15 for an example) is an effective and efficient alternative control method (Senthilkumar 2007). Biological weed control is used against invading plant species that pose a threat to endangered ecosystems and is designed to reduce the competition for nutrients and space by weeds. The aim of biological control of weeds is to decrease, suppress or kill the weed population using host specific insect herbivores or plant pathogens such as fungi, bacteria and viruses (Bailey et al. 2010).

The initial concept for using fungal pathogens in weed control was observed by a farmer in 1890 in the USA with the thistle population controlled by a rust fungus (Wilson 1969). A similar situation was recorded by Morris (1991) who observed that the Australian gall-forming rust fungus Uromycladium tepperianum helps to reduce the infestation of Acacia saligna at over 50 localities in South Africa. Until 1970 biocontrol of weeds was not considered as a solution or alternative to the massive use of chemical herbicides (Pointing and Hyde 2001).

Biological control refers to the planned introduction of an exotic bio control agent for permanent establishment for long term control in an area where weeds are problematic to the natural habitat (Evans 1998; Eilenberg et al. 2001). Fair examples for using fungi in biocontrol of weed are the successful use of the European rust fungus Phragmidium violaceum to control European blackberry (Rubus sp.) in Chile, or the use of Puccinia chondrillina to control
Chondrilla juncea (rush skeleton weed) in Australia, which is considered as the most remarkable successes ever achieved with biocontrol (Kendrick 1992). These rust fungi are obligate biotrophs and cannot mass produce spores (or even grow) in an artificial medium. Therefore, small amounts of natural inoculum were introduced to the area and the weed control was obtained by natural spore production and dispersal (Cullen et al. 1973; Cullen and Delfosse 1985; Kendrick 1992; Espiau et al. 1998).

Inocula of plant pathogens, applied to weeds in a similar manner to synthetic herbicides are called bioherbicides. When fungi are involved, they are referred to as mycoherbicides (Boyetchko et al. 1996). These pathogens usually occur naturally on weeds in localities where they need to be controlled, but not in sufficient amounts. Therefore, fungal inocula are mass produced and sprayed on to the weeds. These mycoherbicide products must undergo a governmentregulated registration process similar to chemical herbicides before they are released for public use (Evans 1998).

Mycoherbicide studies began in the 1940s in several countries with the aim to spread indigenous pathogenic fungal species on target weeds as a control measure (Pointing and Hyde 2001). In 1949, an attempt to control prickly pear cactus using Fusarium oxysporum was unsuccessful (Julien and Griffiths 1998). Kendrick (1992) mentioned another few examples where mass produced fungal propagules are applied as a mycoherbicides. A Colletotrichum gloeosporioides spore suspension was used to control northern joint vetch (Aeschynomene virginica) in rice and soybean fields in the USA. This was the first practical use of a fungus as a mycoherbicide that was later commercialised as "Collego". Another example was water hyacinth, considered to be the worst aquatic weed in the world, but could be controlled by Acremonium zonatum and Cercospora rodmanii. However, during the 1950s, spores of Alternaria cuscutacidae were mass produced and applied to a dodder (Cuscuta sp.) (Wilson 1969, Julien and Griffiths. 1998). In 1963 China developed a mycoherbicide for dodder, using Colletotrichum gloeosporioides cf. cuscutae (Colletotrichum cuscutae) (Auld 1997; Kendrick 1992).

In the 5th edition of "A world catalogue of agents and their target weeds" by Winston et al. (2014), seven fungal species are mentioned that were developed as mycoherbicides and some of these are available as commercial products. According to Walker and Connick (1983) and Auld (1993) dew and temperature are the most important factors for primary infection of pathogen in order to obtain a successful disease development on the target weed. Some researchers have insisted that it is important to consider the time for secondary infection; which lead to a successive distribution of disease in the field (Boyette et al. (1979). According to Hasan and Ayres (1990) and El Morsy (2004), Stagnospora species on Calystegia sepium required 
only 3 weeks for control and Alternaria alternata on water hyacinth took 2 months.

Finding a new active fungal isolate for mycoherbicide production is not an easy task. There are a few important factors that need to be considered before mass production (O'Connell and Zoschke 1996). Mass availability of product, scientific testing for laboratory and field conditions, registration and commercializing procedures are necessary (Evans 1998). Within the past three decades research for improving this technology has increased. Even though the target weeds and phyto-pathogenic species identifications are still ongoing, interesting discoveries have surfaced overtime. According to Gan et al. (2013), the number of candidate genes found from the genomes of Colletotrichum gloeosporioides and Colletotrichum orbiculare, which were predicted to be involved in pathogenesis, showed the potential to be explotied as mycoherbcides. The discovery of genes in these two organisms related to the production of Indole acetic acid (IAA), a component of some of the well established herbicides, (see also chapter 21) showed that these can be converted to mycoherbicides (Gan et al. 2013). Some Phoma species were also considered as a successful candidate for the biocontrol of weeds. The ability of Phoma macrostoma to inhibit the growth of dicot plants was studied (Bailey et al. 2011, 2013; Smith et al. 2015). This fungus was used to control broadleaf weeds in turf systems in Canada and the USA. A registered commercial product of Phoma macrostoma is also available in Canada and USA (Evans et al. 2013).

The secondary metabolites produced by some fungi have been shown to have herbicidal activity. Castro de Souza et al. (2016) found several Diaporthe spp. from the Brazilian Pampa biome that have the ability to produce secondary metabolites with herbicidal activity. The genus Diaporthe is very rich in secondary metabolites, as recently summarised by Chepkirui and Stadler (2017).

Biological control may take several years to take effect and the effectiveness is influenced by a number of factors, such as climatic conditions, geographical region and management practices (Pointing and Hyde 2001). Among these, high initial cost, limited number of natural enemies and uncontrollable dissemination of biological control agents after its release in nature are considered as disadvantages (El-Sayed 2005). Biological control is particularly useful in areas where other conventional control methods are inappropriate, uneconomic or unachievable (Reznik 1996).

Different fungal species act as a promising source for the production of various compounds that can be used as potential herbicides. Since many of these toxins play a key role in the development of plant diseases (Pointing and Hyde 2001); the potential of these chemicals as herbicides can also be explored. When it comes to controlling weeds, herbicide-resistant weeds can be a challenge for conventional control methods. Therefore, there is a potential to find compounds that can act as models for developing herbicides with new modes of action (Castro de Souza et al. 2016). Through in depth studies on the potential of fungi and their products, more environmentally friendly herbicides can be produced for sustainable and eco-friendly control of weeds.

\section{Fungal antagonists used in post-harvest disease control}

There are a myriad of post-harvest applications for fungal antagonistic agents however there are several challenges during the commercialization of these biocontrol strains. At present only two commercial products available as postharvest antagonistic agents with a small market share. Biosave (based on the bacterium Pseudomonas syringae) has been used in the USA to control diseases of sweet potato and potato diseases. "Shemer" (based on the yeast Metschnikowia fructicola) has been applied in Israel commercially to control post-harvest rots of sweet potato and carrot (Droby et al. 2009). Large-scale feasibility tests are warranted before the antagonistic strains are to be applied to fresh commodities. For the successful implementation of biocontrol strategies, the combination of commercial settings, industrial support, and quality control mechanisms to build up the confidence of farmers are all critical factors in the field. Some examples of potential fungi that can be used in post-harvest applications are provided below.

Saccharomycetes: Candida is a genus that comprises some commonly used antagonistic yeasts that can reduce post-harvest decay of several fresh commodities. For example, Candida guilliermondii has been used to control gray mold caused by Botrytis cinerea in nectarines and peach (Tian et al. 2002) and $C$. incommunis is effective against Aspergillus carbonarius and A. niger (which produce ochratoxin A) in grape berries (Bleve et al. 2006). The volatile organic compounds of $C$. intermedia reduce Botrytis fruit rot in strawberries (Huang et al. 2011). Candida oleophila has controlled banana crown rot caused by Colletotrichum musae, Fusarium moniliforme and fungal complexes (Lassois et al. 2008). Droby et al. (2009) applied Candida saitoana solely against Penicillium digitatum and Botrytis cinerea on the post-harvest rots affecting pome and citrus fruits. Candida membranifaciens has reduced post-harvest anthracnose disease caused by $\mathrm{Col}$ letotrichum gloeosporioides in mangos (Kefialew and Ayalew 2008). In vitro application of Pichia anomala (also known as Wickerhamomyces anomalus) has controlled post-harvest decay of apples (Santos et al. 2004) and bunch rot of table grapes (Parafati et al. 2015). In addition, the 
in vivo application of Pichia anomala has reduced crown rot disease in banana (Lassois et al. 2008). Saccharomyces boulardii can induce phytoalexin formation on sorghum and soybean (Stangarlin et al. 2010). Saccharomyces cerevisiae inhibited the activity of Botrytis cinerea (in grapes) by producing volatile compounds (Parafati et al. 2015). In vitro application of Metschnikowia pulcherrima against post-harvest rot of grapes (Bleve et al. 2006, Parafati et al. 2015) was also notably successful.

Other Ascomycota: Aureobasidium pullulans has been used to control bunch rot in table grapes caused by Botrytis cinerea (Parafati et al. 2015). In addition, post-harvest rot of table grapes caused by Monilinia laxa (Schena et al. 2003) were also controlled successfully by this fungus. Penicillium frequentans has been shown to effectively inhibit the growth of Monilinia sp., which causes brown rot in peaches (Guijarro et al. 2007).

Basidiomycota: Cryptococcus magnus can inhibit the mycelial growth of Colletotrichum gloeosporioides in vitro, and controlled post-harvest anthracnose in papaya (de Capdeville et al. 2007). An aqueous extracts from the basidiomes of Lentinula edodes has controlled the growth of Puccinia recondita f. sp. tritici (Fiori-Tutida et al. 2007). In addition, the in vivo application of Pycnoporus sanguineus has controlled the angular leaf spot in beans caused by Pseudocercospora griseola (Viecelli et al. 2009). Aqueous extracts of the basidiomes of Agaricus subrufescens have also shown an antagonism against Puccinia recondita f. sp. tritici. (Fiori-Tutida et al. 2007). However, these basidiomycetes and their extracts have not been proven to be effective in field trials, and the development of methods for effective mass production to attain favorable costs of goods will constitute a serious problem.

\section{Biocontrol of rusts and smuts by antagonistic fungi}

Rust fungi (Uredinales) are one of the largest groups in the Basidiomycota, comprising about 5000-6000 species found on a wide range of hosts, including ferns, gymnosperms, and mono- and dicotyledonous angiosperms (Alexopoulos et al. 1996). Diseases such as coffee leaf rust, Hemileia vastatrix, wheat stem rust, Puccinia graminis, Melampsora leaf rusts of Salicaceae (Populus and Salix) and Cronartium stem rusts of hard pines are causing enormous losses and often making it necessary to replace susceptible crops entirely with non-host species (Littlefield 1981)

Smuts primarily affect grasses viz corn (maize), wheat, sugarcane, barley, oats, forage grasses and sorghum (Feldbrügge et al. 2013). A smut is characterized by spores that accumulate in soot-like masses called sori, which are formed within blisters in seeds, leaves, stems, flower parts, and bulbs (Laurie et al. 2012). The sori usually break up into a black powder that is readily dispersed by the wind. Many smut fungi enter embryos or seedling plants, then develop systemically, and appear externally only when the plants are nearing maturity (Liu et al. 2017a). Currently, the most widely used control method for sugarcane smut disease is the breeding of resistant cultivars (Shen 2002; Wada 2003; Croft et al. 2008; Lwin et al. 2012; Shen et al. 2014). However, its development is constrained by long breeding processes, high costs, and the availability of smutresistant parental lines. Disease attributed to smut fungi could also be controlled by soaking seed canes with chemical fungicides (Olufolaji 1993; Bhuiyan et al. 2012). Another approach is using plant or fungal extracts that inhibit smut pathogen germination and growth (Lal et al. 2009). A large number of fungi have been identified as hyperparasites of rust and smut fungi, which are being used as biocontrol agents worldwide (Gowdu and Balasubramanian 1988; Kranz 1981; Feldbrügge et al. 2013).

Various studies support the ability of certain fungi to control the growth of smuts and rusts. The mechanisms through which biocontrol agents act are antibiosis, secretion of metabolites that are toxic, lytic enzymes, parasitism and competition for nutrients. Figure 3 shows the different mechanisms of antagonistic fungal species action. Biological approaches are gaining popularity, including the use of microbial antagonists (Eckert and Ogawa 1988). Cladosporium species co-exist with rust sori, and some are believed to be invariably hyperparasites of Uredinales (Moricca et al. 1999). Cladosporium uredinicola is a common necrotrophic hyperparasite that can destroy rust hyphae and causes coagulation and disintegration of the cell cytoplasm of Puccinia cestri (Spegazzini 1912), Puccinia (Ellis 1976), Cronartium quercuum (Morgan-Jones and McKemy 1990), Puccinia violae (Traquair et al. 1984) and Puccinia horiana (Srivastava et al. 1985). Moreover, C. uredinophilum was also reported to colonize and destroy Uredo cyclotrauma propagules in Paraguay (Spegazzini 1912). Steyaert (1930) also described C. hemileiae as an effective hyperparasite of coffee rust fungus, Hemileia vastatrix, in Zaire (Democratic Republic of Congo). Powell (1971) reported that $C$. gallicola in galls of Cronartium comandrae on Pinus contorta var. latifolia is parasitic on aeciospores. Hyphae of $C$. gallicola penetrate into the aeciospores of pine gall rust, Endocronartium harknessii (Sutton 1973). Tsuneda and Hiratsuka (1979) investigated C. gallicola and found that it parasitized E. harknessii by both simple contact-disintegrating the cell walls of the spores-and by actual penetration of the spore walls, with or without the formation of appressoria, causing the coagulation and disappearance of the host cytoplasm. 
Hulea (1939) and Rayss (1943) documented a similar phenomenon where $C$. aecidiicola, a common hyperparasite of rusts in Europe and in the Mediterranean area, parasitized E. harknessii on Pinus spp. in California (Byler et al. 1972). Keener (1954) stated that this hyperparasite also drastically parasitized aecia of Puccinia conspicua in Arizona and urediniospores of Melampsora medusae under storage conditions (Sharma and Heather 1980). Moreover, Srivastava et al. (1985) also documented that Puccinia horiana was often regulated by Cladosporium sphaerospermum and $C$. tenuissimum. They were also detected from aeciospores of the two-needle pine stem rust Cronartium flaccidum (Moricca et al. 1999). Other groups of fungi aside from Cladosporium were also reported to act as biocontrol agents. The entomopathogenic and mycoparasitic fungus Lecanicillium lecanii is also known to attack coffee leaf rust, Hemileia vastatrix (Jackson et al. 1997).

Not many commercial biofungicides for rust and smuts based on antagonistic fungi are currently available. Mahmud and Hossain (2016) showed that the BAU-biofungicide (2\%) (Trichoderma based preparation) significantly affected the mycelial growth of Ustilaginoidea virens in an in-vitro test, but this observation remains to be confirmed in greenhouse and field trials.

Kranz (1981) documented more than 80 species of fungi from over 50 genera reported as hyperparasites of rusts. However, this number might be an overestimate due to some taxa being synonyms. Even though this large number of antagonistic fungi on rusts and smuts has been reported, few commercially, improved biofungicides are available for practical application. As in other applications of biocontrol agents (see the above chapters), product formulation is the most critical step of the entire development process (Janisiewicz and Jeffers 1997). The next few years will likely see the increased application of biocontrol agents in agriculture, with particular emphasis on the use of mixtures of antagonists on the same plant organ. This approach may lead to a wider spectrum of activity of the biological treatment or an increase in either the efficacy or consistency of the biological treatment. Furthermore, collaborative work of academic, federal and private sector scientists is necessary to develop more effective and consistent biofungicides.

\section{Enhancing crops and forestry}

In this section, we report on the ways in which fungi are being used or may be used in the future in enhancing plant development within agriculture, forestry and horticulture.

\section{Biofertilizers}

Biofertilizers are produced from organic matter or agroindustrial wastes, which act as substrate for propagation of inoculum of selected microorganisms (Kaewchai et al. 2009). There are two approaches to developing potential biofertilziers: either the application of a single superior species with multifunctions, or groups of microorganisms (consortia) beneficial to plants (Vassilev et al. 2015). Biofertilizers have been used in agriculture, horticulture, landscape restoration, and soil remediation since the late 1980s (Hart and Trevors 2005). The long-term use of biofertilizers is economical and also eco-friendly to plant, animal and human health, and biofertilizers are renewable and low-cost resources which are accessible to marginal and small farmers (Dubey and Maheshwari 2008; Pal et al. 2015). Thus, the use of biofertilizers is recommended over chemical fertilizers. Details regarding biofertilizers such as term, role, types and advantages have been described by Kaewchai et al. (2009), Pal et al. (2015), Vassilev et al. (2015) and Itelima et al. (2018).

Several studies have applied fungal inocula as biofertilizers in greenhouse and/or field trials (Grigera et al. 2007; Rahi et al. 2009; Goetten et al. 2016; Zhang et al. 2016a; Wang et al. 2018c). Mycorrhizal fungi are widely used in agriculture, as they form root symbiotic relationships and provide many benefits to plants, such as improved plant growth and development, increased nutrient uptake and enhanced plant tolerance to disease (Whipps 2004; Liu and Chen 2007; El-Shaikh and Mohammed 2009; Smith et al. 2010; Hernández-Montiel et al. 2013; Goetten et al. 2016; Janoušková et al. 2017). Strains of the genera, such as Alternaria, Aspergillus, Chaetomium, Fusarium, Penicillium, Serendipita (Piriformospora), Phoma, and Trichoderma have been reported as plant growth promoting fungi (Soytong et al. 2001; Muhammad et al. 2009; Salas-Marina et al. 2011; Varma et al. 2012; Bitas et al. 2015; Murali and Amruthesh 2015; Zhang et al. 2016a; Zhou et al. 2018). Examples of the use of fungal inocula treatments on plants are provided in Table 8. These potential plant growth-promoting fungi can be further researched and developed as potent fungal biofertilizers.

Numerous commercial fungal biofertilizer products have been manufactured globally and are available on the market today. There are various formulation types, such as granules, wettable powder, pellets and liquids, which comprise one or multiple fungal inocula. Aspergillus, Chaetomium, Penicillium and Trichoderma species have been used in biofertilizer products. For example, Ketomium ${ }^{\circledR}$ has been developed and improved from strains of Chaetomium spp. in pellet and powder form. The product was used in greenhouse and field trials of tomato, 
Table 8 Examples for the use of fungal (and oomycete) inocula treated on plants

\begin{tabular}{|c|c|c|c|}
\hline Fungal inoculum & Treated plant & Result & References \\
\hline Arbuscular mycorrhiza & Zea mays & $\begin{array}{l}\text { Active during the reproductive growth stages and may } \\
\text { benefit high productivity of maize crops by facilitating P } \\
\text { uptake }\end{array}$ & Grigera et al. (2007) \\
\hline Arbuscular mycorrhiza & Citrullus lanatus & $\begin{array}{l}\text { Reduced replant problems through effective modification } \\
\text { of the soil microbota structure, and by increasing the soil } \\
\text { enzyme activities }\end{array}$ & Zhao et al. (2010) \\
\hline $\begin{array}{l}\text { Arbuscular mycorrhiza } \\
\text { (Rhizophagus clarus and } \\
\text { Claroideoglomus etunicatum) }\end{array}$ & $\begin{array}{l}\text { Woody plant } \\
\text { seedlings of } \\
\text { various plants }\end{array}$ & $\begin{array}{l}\text { - Increased root colonization of all woody plant seedlings } \\
\text { - Increased plant height and stem diameter of } L \text {. divaricata, } \\
\text { C. robustum and C. fissilis } \\
\text { - Increased shoot biomass growth of } L \text {. divaricata, } C \text {. } \\
\text { robustum, G. gardneriana and } C \text {. fissilis } \\
\text { - Increased shoot phosphorus of } C \text {. robustum, S. } \\
\text { terebinthifolius and G. gardneriana }\end{array}$ & Goetten et al. (2016) \\
\hline Aspergillus niger (CS-1) & Wheat & $\begin{array}{l}\text { Promoted plant growth by increasing the fresh and dry } \\
\text { mass of wheat per plant in pot experiments }\end{array}$ & Wang et al. (2018c) \\
\hline Discosia sp. (HKUCC 6626) & $\begin{array}{l}\text { Zea mays, Pisum } \\
\text { sativum, Cicer } \\
\text { arietinum }\end{array}$ & $\begin{array}{l}\text { Increased root length, shoot length and dry matter in the } \\
\text { test plants over the uninoculated control under the } \\
\text { controlled environment }\end{array}$ & Rahi et al. (2009) \\
\hline $\begin{array}{l}\text { Endophytic strains of Fusarium } \\
\text { tricinctum (RSF-4L) and } \\
\text { Alternaria alternata (RSF-6L) }\end{array}$ & Rice plants & $\begin{array}{l}\text { Enhanced growth attributes, including increased root-shoot } \\
\text { lengths, chlorophyll contents, and biomass }\end{array}$ & Khan et al. (2015a) \\
\hline $\begin{array}{l}\text { Penicillium sp., Trichoderma sp., } \\
\text { (Pythium sp. (Oomycete) }\end{array}$ & Pearl millet seeds & $\begin{array}{l}\text { - Penicillium sp. at 5\% (w/w) concentration recorded } \\
\text { highest seed germination of } 92 \% \text { and } 1701.9 \text { seedling } \\
\text { vigor } \\
\text { - Penicillium sp. at 5\% (w/w) and Pythium sp. at } 10 \% \text { (w/ } \\
\text { w) showed maximum disease protection of } 67 \% \text { and } 61 \% \\
\text { respectively against downy mildew disease of pearl millet } \\
\text { - Penicillium sp. and Trichoderma sp. recorded highest } \\
\text { disease protection of } 71 \% \text { and } 66 \% \text {, respectively under } \\
\text { greenhouse conditions }\end{array}$ & Murali et al. (2012) \\
\hline Purpureocillium lilacinum & Tomato seeds & $\begin{array}{l}\text { Increased of the percentage of tomato seed germination } \\
\text { from } 71 \text { to } 85 \% \text { after } 48 \mathrm{~h}\end{array}$ & Cavello et al. (2015) \\
\hline Trichoderma longibrachiatum & Wheat seeds & $\begin{array}{l}\text { Increased wheat seedling height and root length, compared } \\
\text { to the } \mathrm{NaCl} \text { stress treatment }\end{array}$ & Zhang et al. (2016a) \\
\hline
\end{tabular}

corn, rice, pepper, citrus, durian, bird of paradise and carnation plants in Thailand (Soytong et al. 2001). Plants treated with Ketomium ${ }^{\circledR}$ showed better plant growth and higher yield than non-treated control plants. In addition, Ketomium $^{\circledR}$ had the ability to control Phytophthora sp., causing citrus root rot in the field. Other examples of fungal biofertilizer products are given in Table 8 .

Biofertilizers increase the uptake of nutrients from the soil or atmosphere, and produce bioactive compounds, enzymes and hormones which stimulate plant growth and enhance root growth (Chi et al. 2010; Abdel-Fattah et al. 2013; Pal et al. 2015). Fungal biofertilizers are able to solubilize and mobilize unavailable organic and inorganic forms of phosphorus into soluble forms, making them available to plants. For example, Aspergillus niger was mixed with Bacillus megaterium to form phosphate solubilizing microorganisms. These microorganisms were applied as biofertilizers in India (Pal et al. 2015). Arbuscular mycorrhizae have been used as phosphate mobilizing biofertilizers (Zhang et al. 2018). Biofertilizers play an important role in the recycling of plant nutrients and in enhancing the rate of compost degradation (Pal et al. 2015). Some biofertilizers act as antagonists and suppress the incidence of soil borne plant pathogens while helping in the biocontrol of plant diseases (Thamer et al. 2011; Pal et al. 2015). 
Fungal derived stimulants, or elicitors, are fungi or fungal compounds that enhance the production of secondary metabolites, or elicit growth or immune response in a target plant species upon application. Plant responses include the upregulation of genes involved in plant defense, as well as the increased production of antimicrobial compounds, lignin, secondary metabolites, and certain proteins (Vassilev et al. 2015). Potential uses for such elicitors include the enhanced production of commercially valuable compounds/metabolites, or the artificial enhancement of plant defenses when pathogens are detected (Radman et al. 2003). A novel approach for the use of elicitors is to incorporate them with immobilized stimulants, such as with arbuscular mycorrhizal inoculum. Additionally, plantderived elicitors, which enhance the growth and development of beneficial fungi such as arbuscular mycorrhizae, also show promise in advancing this field of study (Akiyama et al. 2005; Besserer et al. 2006). Elicitors have a high potential for enhancing plant productivity and improving plant defenses against pathogens, and given that elicitors can be used in combination with other types of biofertilizers, they hold much potential for wide scale application in the future.

Fungal biofertilizers are applied on a very small scale in agriculture as compared to chemical fertilizers due to their limited shelf life and slower rate of effect. Olivian et al. (2004) reported using sterilized peat as solid support for Fusarium oxysporum inoculation, storing this admixture at room temperature without loss of activity. Growth and formulations based on recycling agro-industrial wastes can be expected to employ nitrogen-fixing and other microorganisms with different characteristics, such as biocontrol, P-solubilization, lignocellulolytic activity. For example, combinations between Trichoderma spp. and P-solubilizing fungi can be cultured based on agroindustrial-wastes, leading to mineralization of the matrix/substrate by the combined enzyme actions. We could apply immobilization of fungal cells together with enhanced biotechnology and in combination with elicitors. Immobilized cell technologies permit the use of two and more microorganisms, which result in highly effective synergies benefiting all the organisms involved, including the plants (Vassilev et al. 2015). In order to effectively implement the use and gain the full benefits of biofertilizers, an integrated approach engaging a variety of mechanisms should be considered. Such an approach could be tailored to suit specific industry needs and target defined outcomes, such as improved growth, upregulation of key metabolites, or enhanced plant defenses.

\section{Arbuscular mycorrhizae as biofertilizers}

Ectomycorrhizal association describes a structure that results from a mutualistic symbiosis between the roots of higher plants and root-inhabiting fungi. Within this symbiotic relationship, the role of the fungi is to help the host plants take up water and nutrients, receiving plant-derived carbohydrates from photosynthesis in return. About 6000 plant species in 145 genera and 26 families (approximately 5600 angiosperms and 285 gymnosperms) have been estimated to possess ectomycorrhizal symbiotic fungal partners (Brundrett 2009; Tedersoo et al. 2010). Ectomycorrhizal association helps both the fungi and their host plants to overcome environmental stresses caused by low nutrients, drought, disease, extreme temperatures and heavy metal contamination (Smith and Read 2008; Courty et al. 2010; Kipfer et al. 2012; Heilmann-Clausen et al. 2014). Moreover, ectomycorrhizae can improve soil structure and nutrients; protect the plants against root pathogens; promote plant growth by producing phytohormones; and increase the photosynthetic rate of the plants (Splivallo et al. 2009; Ramachela and Theron 2010; Makita et al. 2012). Ectomycorrhizae are dominated by members of the Basidiomycota, some Ascomycota, and, rarely, Mucoromycota (Taylor and Alexander 2005; Rinaldi et al. 2008; Tedersoo et al. 2010). Generally, ectomycorrhizae produce reproductive fruiting bodies appearing above- or below-ground that are essential to the food webs of forest ecosystems and their spore dispersal (Rinaldi et al. 2008; Wilson et al. 2011).

Plant seedling regeneration and restoration are of pivotal interest to forestry, but the survival of seedlings is often poor both in nurseries and natural plantation areas, especially in mine spoils, polluted areas, and other treeless areas. Therefore, the main purpose for the application of ectomycorrhizae is to improve the survival and growth of seedlings. The potential advantages of ectomycorrhizal association in nurseries are not only the positive growth responses of the seedlings, but also a reduction of fertilization costs in an environmentally friendly manner. The role of ectomycorrhizae in forest establishment and recovery has been well-established. Numerous studies on the ectomycorrhizae inoculation of seedlings have shown increases in plant growth and productivity, the viability of seedlings, and seedling establishment on a forest restoration programs (Teste et al. 2009; Dalong et al. 2011; Brearley et al. 2016; Velmala et al. 2018). Ectomycorrhizae are particularly important for the growth of economically important trees, including species of beech (Fagus), dipterocarps (Dipterocarpus and Shorea), eucalyptus (Eucalyptus), oak (Quercus and Castanopsis), pine 
(Pinus) and spruce (Picea) (Tennakoon et al. 2005; Flykt et al. 2008; Dalong et al. 2011; Kayama and Yamanaka 2016). Cenocococum, Pisolithus, Laccaria, Rhizopogon, Russula, Scleroderma and Thelephora species have been shown to increase the rate of survival and growth of eucalyptus, pine and oak seedlings in both plantation and reforestation programs (Fig. 16) (Chen et al. 2006; Jha et al. 2008; Cram and Dumroese 2012; Kipfer et al. 2012; Zong et al. 2015).

Generally, three main types of ectomycorrhizal inoculants-soil, fruiting body/spore and vegetative myceliumhave been used in nurseries. Forest soil was used as a source of indigenous ectomycorrhizal fungi in an inoculation experiment mixed with planting substrates (Kaewgrajang et al. 2013; Dulmer et al. 2014; Restrep-Liano et al. 2014; Livne-Luzon et al. 2017). This method is still used in many parts of the world, particularly in developing countries. However, the use of forest soil inoculants has the major disadvantage that the ectomycorrhizal composition is unknown. Moreover, it requires large amounts of soil and hence risks introducing plant pathogens and weeds exits. Fruiting bodies/spores of various ectomycorrhizae are easily obtained from natural forests and can be easily applied to plant seedlings as inoculants. The variety of application methods include mixing with sand, clay, or vermiculite carrier before being added to planting substrate or soil, suspension in water and drenching or irrigating, spraying, and encapsulation or coating onto seeds. Ectomycorrhizae that are "gasteromycetes" (puffball fungi) with conspicuous basidomes are better sources than the gilled fungi if large numbers of spores are required, as they are easier to collect and use. For instance, species of the genera Pisolithus, Rhizopogon and Scleroderma produce a large quantity of spores, and the approximate spore concentration in a seedling inoculation may range from $10^{5}-10^{7}$ spores/ml (Chen et al. 2006; Bruns et al. 2009; Rai and Varma 2011; Aggangan et al. 2013). Most previous studies resulted in acceptable levels of ectomycorrhizal association, improved seedling growth of pines in the nursery, and improved outplanting success following inoculation with Pisolithus and Rhizopogon spores (Bruns et al. 2009; Dalong et al. 2011).

There are of course limitations to fruiting body/spore inoculants: only those ectomycorrhizal species able to produce large numbers of fruiting bodies and spores can be used, and there may be a concern about the compatibility and efficiency of ectomycorrhizae to the plant species to be cultivated. As an alternative, vegetative mycelial inoculants obtained from pure cultures of ectomycorrhizae may be prepared from a pure culture using different methods, e. g. using mycelial suspensions and substrate carriers such as forest litter, cereal grains, peat moss, vermiculite, and alginate-beads (de Oliveira et al. 2006; Rossi et al. 2007; Lee et al. 2008a, b; Restrep-Liano et al. 2014; Kayama and Yamanaka 2016; Kumla et al. 2016). This inoculant type

Fig. 16 Arbuscular mycorrhizae inoculum production. a Pot culture of sorghum and maize, b on-farm inoculum production using leaf litter compost and agricultural wastes; c In vitro production with root organ culture; $\mathbf{d}$ newly produced Funneliformis mosseae spores attached to Ri T-DNA transformed carrot roots

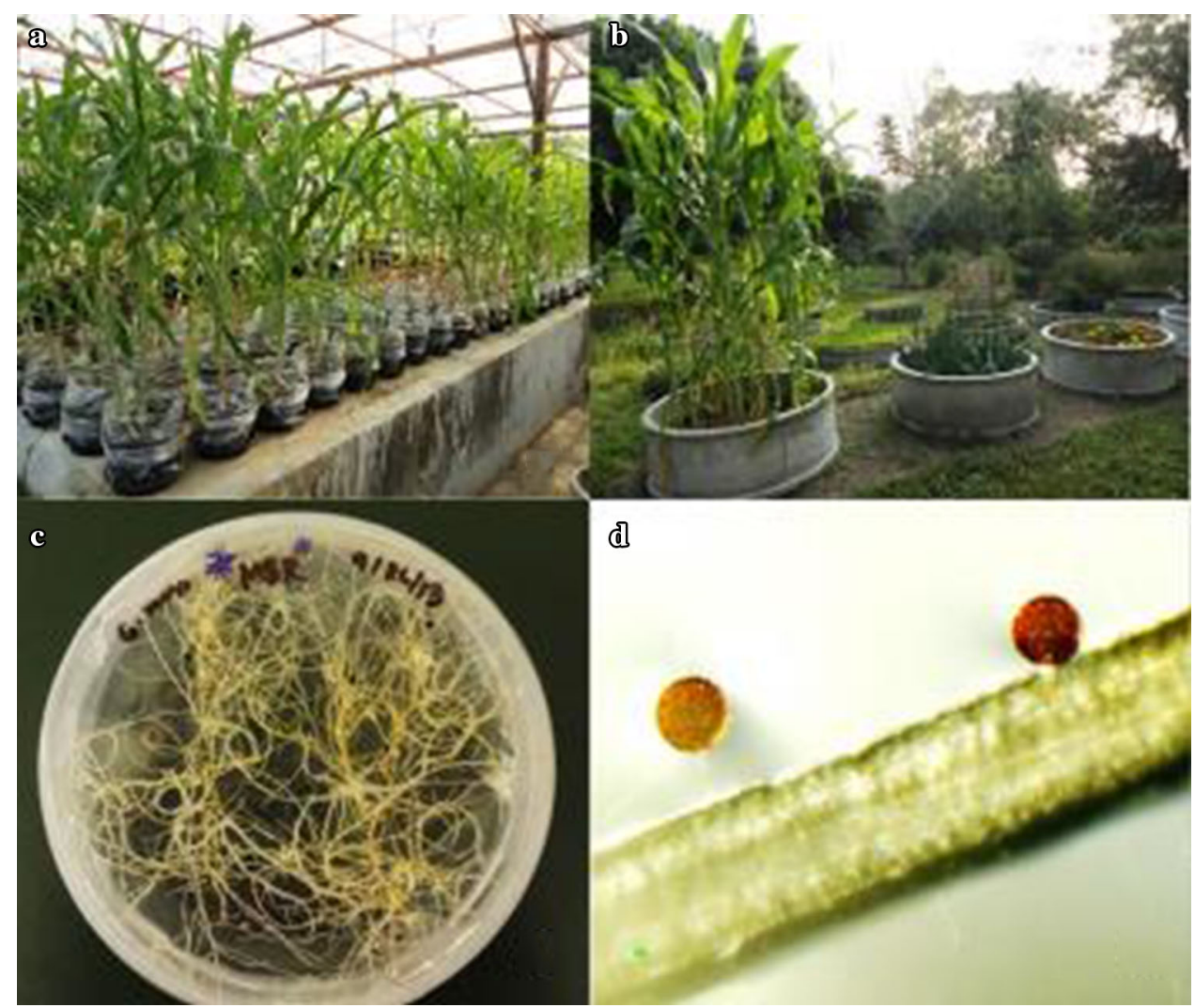


has proven to be the most suitable method because of their efficiency in promoting plant growth by selected fungal isolates. However, optimal conditions, including nutrition, temperature and substrate carrier, must always be established empirically for large-scale production.

Several commercial ectomycorrhizal products have been developed. For instance, the commercial mycelial inoculants of MycoRhiz ${ }^{\circledR}$, Ectomycorrhiza Spawn ${ }^{\circledR}$, Somycel PV and Mycobead $^{\circledR}$ are available. BioGrow Blend ${ }^{\circledR}$, MycoApply ${ }^{\circledR}$-Ecto, Ectovit ${ }^{\circledR}$ and Mycor Tree ${ }^{\circledR}$ Ecto-Injectable are commercially available products with ectomycorrhizae spores. The commercial products produced by mixing endomycorhizae and ectomycorrhizae spores are MycoApply ${ }^{\circledR}$-Endo/Ecto, BioOrganic ${ }^{\mathrm{TM}}$ Mycorrhizal Landscape Inoculant and Mycoke ${ }^{\circledR}$ Pro ARBOR·WP. In order to apply ectomycorrhizae in forestry, it is necessary to select ectomycorrhizal isolates of high compatibility and efficiency in the colonization of the target plant species. Inoculant types, as well as inoculation protocols and skills in nursery practices, lead to the success of an inoculation program under the proper environmental conditions in the plantation site.

The potential for arbuscular mycorrhizae to increase crop yields has been known for decades, but there are few published studies demonstrating the effectiveness of the large-scale inoculation of globally important crops, especially in the tropics where population growth is high (Rodriguez and Sanders 2015). Therefore, researchers need to study large-scale arbuscular mycorrhizae application to crops in the tropics where phosphate bioavailability is low and the application of arbuscular mycorrhizae has the strongest potential to increase food production and reduce the need to apply phosphate fertilizers (Ceballos et al. 2013). Manufacturers should ensure their arbuscular mycorrhizae products are free from other microorganisms and ensure product quality and sufficient weight for cheap transport. Farmers should have easy access to arbuscular mycorrhizae products, correctly apply them to the crops, and know how to produce on-farm arbuscular mycorrhizae inoculum for sustainable agriculture.

\section{Application of ectomycorrhizal fungi in forestry}

Ectomycorrhizal association describes a structure that results from a mutualistic symbiosis between the roots of higher plants and root-inhabiting fungi. Within this symbiotic relationship, the role of the fungi is to help the host plants take up water and nutrients, receiving plant-derived carbohydrates from photosynthesis in return. About 6000 plant species in 145 genera and 26 families (approximately 5600 angiosperms and 285 gymnosperms) have been estimated to possess ectomycorrhizal symbiotic fungal partners (Brundrett 2009; Tedersoo et al. 2010). Ectomycorrhizal association helps both the fungi and their host plants to overcome environmental stresses caused by low nutrients, drought, disease, extreme temperatures and heavy metal contamination (Smith and Read 2008; Courty et al. 2010; Kipfer et al. 2012; Heilmann-Clausen et al. 2014). Moreover, ectomycorrhizae can improve soil structure and nutrients; protect the plants against root pathogens; promote plant growth by producing phytohormones; and increase the photosynthetic rate of the plants (Splivallo et al. 2009; Ramachela and Theron 2010; Makita et al. 2012). Ectomycorrhizae are dominated by members of the Basidiomycota, some Ascomycota, and, rarely, Mucoromycota (Taylor and Alexander 2005; Rinaldi et al. 2008; Tedersoo et al. 2010). Generally, ectomycorrhizae produce reproductive fruiting bodies appearing above- or below-ground that are essential to the food webs of forest ecosystems and their spore dispersal (Rinaldi et al. 2008; Wilson et al. 2011).

Plant seedling regeneration and restoration are of pivotal interest to forestry, but the survival of seedlings is often poor both in nurseries and natural plantation areas, especially in mine spoils, polluted areas, and other treeless areas. Therefore, the main purpose for the application of ectomycorrhizae is to improve the survival and growth of seedlings. The potential advantages of ectomycorrhizal association in nurseries are not only the positive growth responses of the seedlings, but also a reduction of fertilization costs in an environmentally friendly manner. The role of ectomycorrhizae in forest establishment and recovery has been well-established. Numerous studies on the ectomycorrhizae inoculation of seedlings have shown increases in plant growth and productivity, the viability of seedlings, and seedling establishment on a forest restoration programs (Teste et al. 2009; Dalong et al. 2011; Brearley et al. 2016; Velmala et al. 2018). Ectomycorrhizae are particularly important for the growth of economically important trees, including species of beech (Fagus), dipterocarps (Dipterocarpus and Shorea), eucalyptus (Eucalyptus), oak (Quercus and Castanopsis), pine (Pinus) and spruce (Picea) (Tennakoon et al. 2005; Flykt et al. 2008; Dalong et al. 2011; Kayama and Yamanaka 2016). Cenocococum, Pisolithus, Laccaria, Rhizopogon, Russula, Scleroderma and Thelephora species have been shown to increase the rate of survival and growth of eucalyptus, pine and oak seedlings in both plantation and reforestation programs (Fig. 17) (Chen et al. 2006; Jha et al. 2008; Cram and Dumroese 2012; Kipfer et al. 2012; Zong et al. 2015).

Generally, three main types of ectomycorrhizal inoculants - soil, fruiting body/spore and vegetative myceliumhave been used in nurseries (Fig. 18a). Forest soil was used as a source of indigenous ectomycorrhizal fungi in an 
Fig. 17 Application of Pisolithus albus in eucalyptus (Eucalyptus camaldulensis) seedlings after 3 months of inoculation (a). T1 Pisolithus inoculation experiment. $T 2$ Nutrient solution experiment. T3 Uninoculated experiment. Ectomycorrhizal root tip of Pisolithus albus (b). Cross section of ectomycorrhizal root tip of E. camaldulensis showed mantle sheath $(\mathrm{M})$ and Hartig net (arrowed). Scale bar $\mathrm{B}=1 \mathrm{~mm}, \mathrm{C}=20 \mu \mathrm{m}$
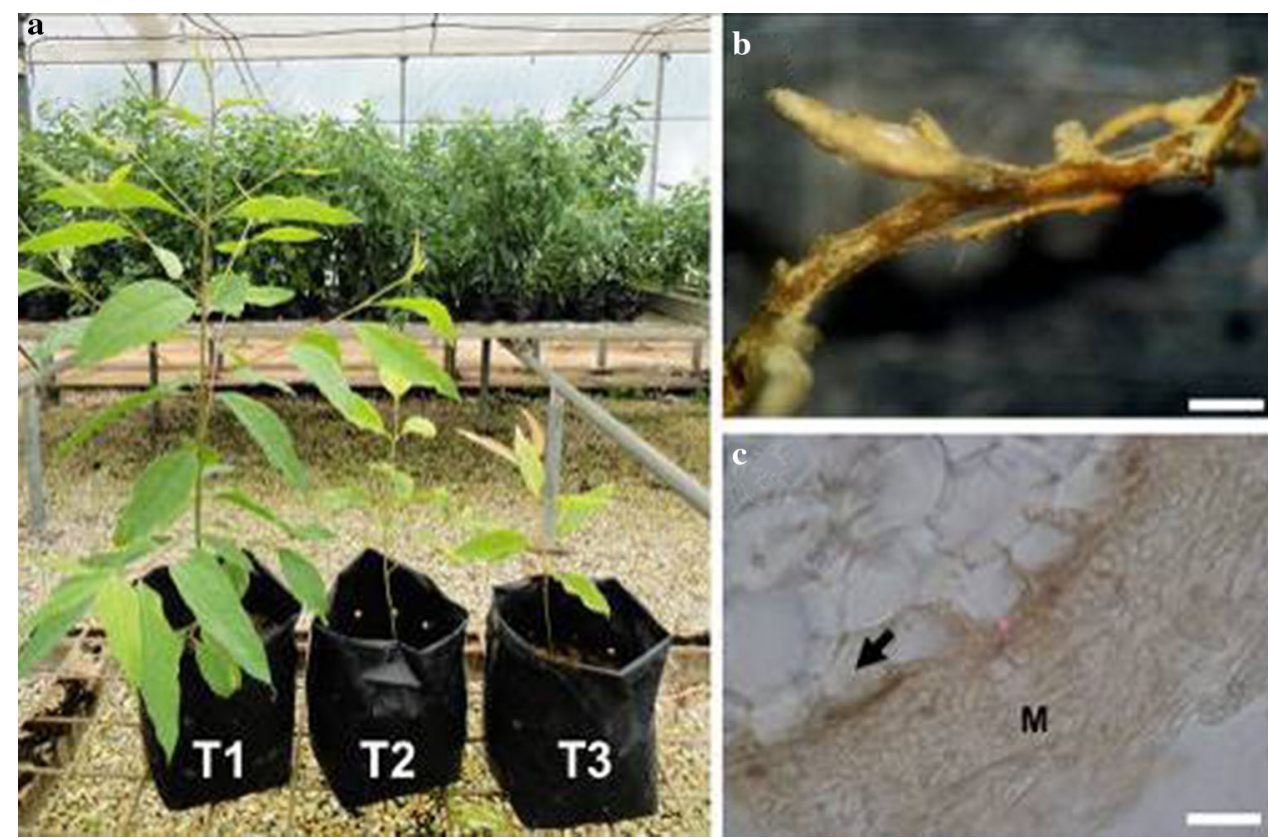

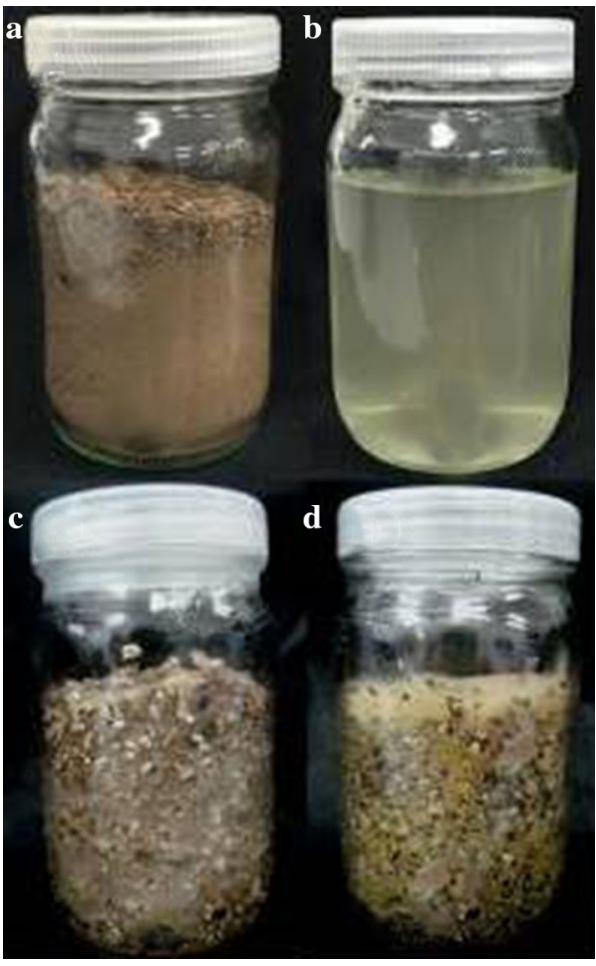

Fig. 18 Inoculant types of ectomycorrhizae (Pisolithus orientalis). a Soil inoculant, b spore suspension inoculant, c, d vegetative mycelial inoculants

inoculation experiment mixed with planting substrates (Kaewgrajang et al. 2013; Dulmer et al. 2014; RestrepLiano et al. 2014; Livne-Luzon et al. 2017). This method is still used in many parts of the world, particularly in developing countries. However, the use of forest soil inoculants has the major disadvantage that the ectomycorrhizal composition is unknown. Moreover, it requires large amounts of soil and hence risks introducing plant pathogens and weeds exits. Fruiting bodies/spores of various ectomycorrhizae are easily obtained from natural forests and can be easily applied to plant seedlings as inoculants. The variety of application methods include mixing with sand, clay, or vermiculite carrier before being added to planting substrate or soil, suspension in water and drenching or irrigating, spraying, and encapsulation or coating onto seeds. Ectomycorrhizae that are "gasteromycetes" (puffball fungi) with conspicuous basidomes are better sources than the gilled fungi if large numbers of spores are required, as they are easier to collect and use. For instance, species of the genera Pisolithus, Rhizopogon and Scleroderma produce a large quantity of spores, and the approximate spore concentration in a seedling inoculation may range from $10^{5}-10^{7}$ spores $/ \mathrm{ml}$ (Chen et al. 2006; Bruns et al. 2009; Rai and Varma 2011; Aggangan et al. 2013). Most previous studies resulted in acceptable levels of ectomycorrhizal association, improved seedling growth of pines in the nursery, and improved outplanting success following inoculation with Pisolithus and Rhizopogon spores (Bruns et al. 2009; Dalong et al. 2011).

There are of course limitations to fruiting body/spore inoculants: only those ectomycorrhizal species able to produce large numbers of fruiting bodies and spores can be used, and there may be a concern about the compatibility and efficiency of ectomycorrhizae to the plant species to be cultivated. As an alternative, vegetative mycelial inoculants obtained from pure cultures of ectomycorrhizae may be prepared from a pure culture using different methods, e. 
g. using mycelial suspensions and substrate carriers such as forest litter, cereal grains, peat moss, vermiculite, and alginate-beads (de Oliveira et al. 2006; Rossi et al. 2007; Lee et al. 2008a, b; Restrep-Liano et al. 2014; Kayama and Yamanaka 2016; Kumla et al. 2016). This inoculant type has proven to be the most suitable method because of their efficiency in promoting plant growth by selected fungal isolates. However, optimal conditions, including nutrition, temperature and substrate carrier, must always be established empirically for large-scale production.

Several commercial ectomycorrhizal products have been developed In order to apply ectomycorrhizae in forestry, it is necessary to select ectomycorrhizal isolates of high compatibility and efficiency in the colonization of the target plant species. Inoculant types, as well as inoculation protocols and skills in nursery practices, lead to the success of an inoculation program under the proper environmental conditions in the plantation site.

\section{Use of orchid mycorrhizae and endophytes in biotechnology}

Orchidaceae is one of the largest families of flowering plants with over 700 genera and 25,000 species (Dearnaley 2007; Sathiyadash et al. 2012). Orchids are found in a wide range of habitats and may grow autotrophically or heterotrophically (Sathiyadash et al. 2012; Tan et al. 2014; Fochi et al. 2017). Orchids are economically very important and their sales represent around $8 \%$ of the world floriculture trade. The economically most important genera are Cymbidium, Dendrobium, and Phalaenopsis (Dearnaley 2007, 2016; Chugh et al. 2009; Emsa-art et al. 2018). Some orchids, such as Gastrodia (Griesbach 2002; Dearnaley 2007), Dendrobium officinale and D. nobile are used as natural medicines ( $\mathrm{Li}$ et al. 2009). Furthermore, the economically most important orchid products are the flavours derived from some species of the genus Vanilla, which are grown at a large scale and used in food and drinks (Dearnaley 2007; Gonzalez-Chavez et al. 2018).
Most orchids rely on mycorrhizal fungi for survival, as they are essential for seed germination and early plant growth (Sathiyadash et al. 2012; Fochi et al. 2017). Different fungal symbiotic mycorrhizae have been recorded from orchids (Table 9). Orchid associated non-mycorrhizal endophytic fungi have been investigated via healthy plant organs including leaves, roots and stems (Ma et al. 2015a), whereas mycorrhizal fungi are generally isolated from root tissues (Tan et al. 2014; Ma et al. 2015a, b). Non-mycorrhizal endophytic fungi represent over 110 genera, including Sordariomycetes (Neonectria, Trichoderma, $\mathrm{Ni}$ grospora, Pestalotiopsis) and Dothideomycetes (Cercospora, Lasiodiplodia, Phyllosticta) (Ma et al. 2015a, b). Dark septate endophytes isolated from Dendrobium and Leptodontidium sp. enhanced seedling development of Dendrobium nobile by forming peloton-like structures in the cortical cells of the orchid (Hou and Guo 2009; Ma et al. 2015a, b). Fusarium species promoted seed germination of Cypripedium and Platanthera orchids (Ma et al. 2015a, b). The endophyte Umbelopsis nana isolated from Cymbidium spp., promoted growth of Cymbidium hybridum (Ma et al. 2015a, b).

Many epiphytic and terrestrial orchids produce minute seeds with minimal nutrient reserves, and lack nutrients for seed germination and development in the early growth stage (Cameron et al. 2006, Sathiyadash et al. 2012; Tan et al. 2014). After germination, orchid seeds produce a protocorm (a preseedling stage/ early stage of the plant) that lacks chlorophyll (Leake 2004; Sathiyadash et al. 2012; Fochi et al. 2017). Protocorms grow in complete dependence on fungal symbionts for nutrients and organic carbon supply (Cameron et al. 2006; Dearnaley 2007). Orchid seedlings develop photosynthetic leaves later and then mature roots are colonized by mycorrhizal fungi (Cameron et al. 2006; Smith and Read 2008; Fochi et al. 2017). The protocorm and mature roots cells are colonized by intracellular fungal coils (pelotons) (Fig. 19) (Dearnaley 2007; Dearnaley et al. 2016; Fochi et al. 2017).

Orchid mycorrhizal associations are useful in the floriculture trade, as they stimulate seed germination and

Table 9 Fungal symbionts associated with different orchid species

\begin{tabular}{|c|c|c|c|}
\hline $\begin{array}{l}\text { Fungal symbionts } \\
\text { (Basidiomycota) }\end{array}$ & Orchid species & Locality & References \\
\hline Rhizoctonia & $\begin{array}{l}\text { Gastrochilus acaulis, Nervilia prainiana and Polystchya } \\
\text { concreta }\end{array}$ & India & $\begin{array}{l}\text { Senthilkumar (2003) Sathiyadash et al. } \\
\text { (2012) }\end{array}$ \\
\hline Ceratobasidium & Zeuxine strateumatica & India & Kumar and Kaushik (2004) \\
\hline \multirow[t]{3}{*}{ Tulasnella } & Amerorchis rotundifolia & Worldwide & Zelmer et al. (1996) \\
\hline & Dactylorhiza majalis & & Kristiansen et al. (2001) \\
\hline & Neuwiedia veratrifolia & & Kristiansen et al. (2004) \\
\hline
\end{tabular}


Fig. 19 a, b Mycelial coils (pelotons, seen as brown areas) in the root section of Cymbidium lowianum collected from the orchid nursery at Queen Sirikit Botanical Garden in Chiang Mai, Thailand, in November 2008
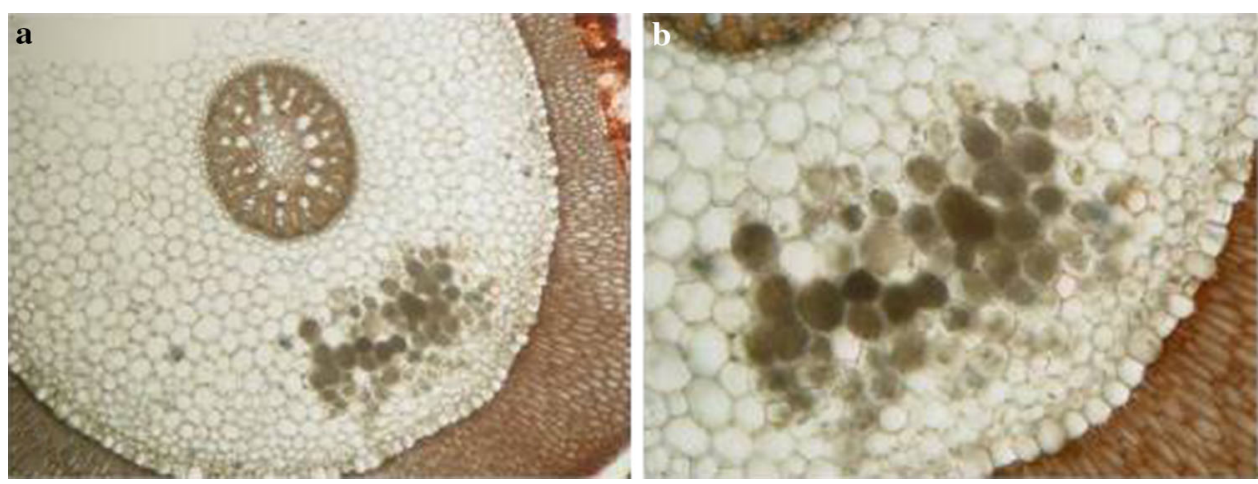

Fig. 20 Scheme illustrating the methodology of orchid seed propagation using mycorrhizal fungi (in-vitro conditions)

(Nontachaiyapoom et al. 2010; Tan et al. 2014). a Effect of mycorrhizal fungi on germination of orchid seeds. b Effect of mycorrhizal fungi on growth of orchid seedlings

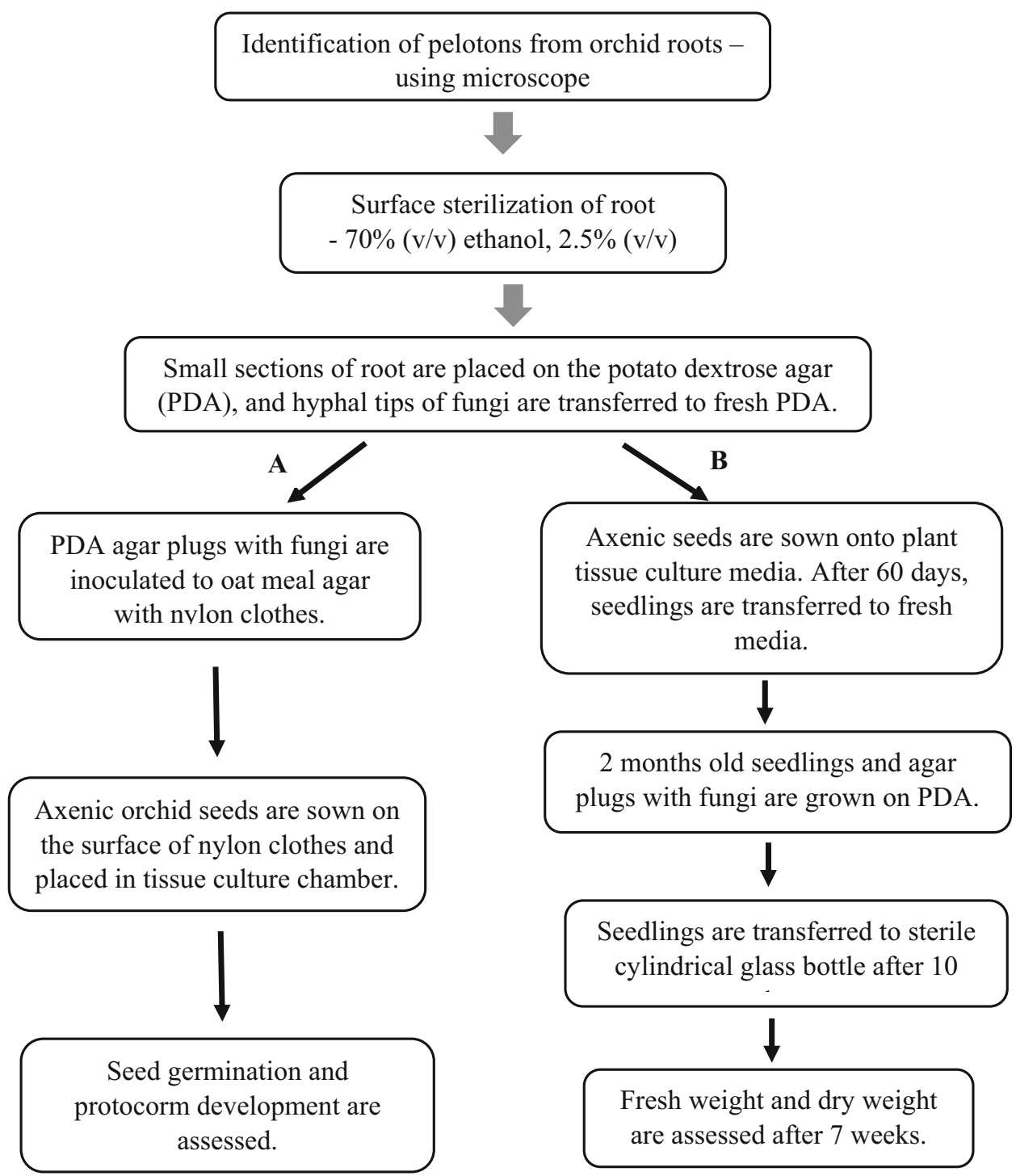

propagate orchids (Tan et al. 2014). A brief methodology for the inoculation of mycorrhizal fungus (Tulasnella sp.) to orchids according to Nontachaiyapoom et al. (2010) and Tan et al. (2014) is represented in a flow chart (Fig. 20). A
Tulasnella sp. isolated from roots of Dendrobium nobile facilitates significantly higher seed germination of $D$. officinale than that of the control (without inoculation of Tulasnella sp.) (Tan et al. 2014). In addition, Tulasnella sp. 
Table 10 Different stages of orchid seed germination (Tan et al. 2014)

\begin{tabular}{ll}
\hline Stage & Description \\
\hline 0 & No germination, viable embryo \\
1 & Enlarged embryo, production of rhizoid(s) (=germination) \\
2 & Continued embryo enlargement, rupture of testa, further \\
& production of rhizoids \\
3 & Appearance of protomeristem \\
4 & Emergence of first leaf \\
5 & Elongation of first leaf \\
\hline
\end{tabular}

promotes seed development up to stage 5 (Table 10), while the control without the fungus developed only to stage 2 (Table 10) (Tan et al. 2014). However, fungi isolated from orchid plant roots do not always exhibit functional symbiotic associations with the orchid plant (Dearnaley 2007). Microscopic observations of orchid root sections might be useful to confirm the presence of intracellular fungal mycelium (Nontachaiyapoom et al. 2010; Emsa-art et al. 2018). Furthermore, it is important to evaluate seedling growth of mycorrhizal inoculated orchids under natural conditions (Tan et al. 2014).

Orchid mycorrhizal fungi are also important for controlling disease in floriculture trade (Yoder et al. 2000; Emsa-art et al. 2018). Inoculation of orchid mycorrhizal fungi may enhance plant immunity against pathogenic diseases (Wu et al. 2011; Emsa-art et al. 2018). For example, soft rot disease is one of the most devastating diseases caused by Dickeya spp., which kills orchids or causes spots/scars on leaves and flowers (Liau et al. 2003; Emsa-art et al. 2018). One recent study showed that soft rot development in mycorrhizal fungi inoculated orchids was significantly reduced compared to that of non-mycorrhizal fungi inoculated orchids in greenhouse conditions. Phalaenopsis is a popular potted plant species that was used for the study by Emsa-art et al. (2018). A brief overview of the methodology of inoculation of mycorrhizal fungi (Tulasnella deliquescens) to Phalaenopsis to control pathogenic Dickeya is presented in Fig. 21.

Several commercial products containing mycorrhizal inoculants exist. These inoculants are available for sale in liquid and powder form for easy and effective usage. Most of these products are organic fertilizers inoculated with mycorrhizae spores and with vitamins, minerals, and nutrients to help bolster the fertility and biological activity of the soil (see Table 11). Mycorrhizal fungi are useful in orchid conservation (Tan et al. 2014). Orchids are a highly diverse plant family and many species may face extinction threats (Reiter et al. 2016) because of habitat loss and overexploitation of attractive species (Dearnaley 2007). With this decline of orchid diversity, it is now an urgent

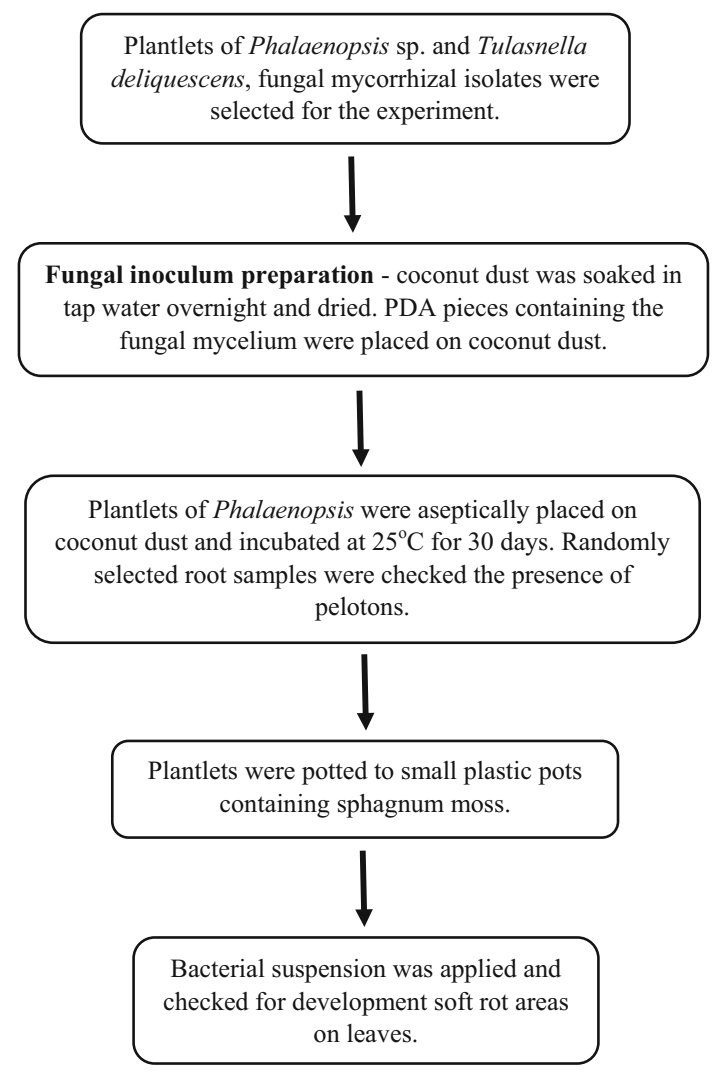

Fig. 21 Scheme illustrating the methodology for the inoculation of mycorrhizal fungi, Tulasnella deliquescens to the orchid Phalaenopsis to control pathogenic bacteria (Emsa-art et al. 2018)

requirement to encourage research on the reintroduction of endangered species to natural habitats (Reiter et al. 2016).

\section{Growth promoting hormones from fungi}

Fungi live in diverse habitats and have adapted to ecological niches, including plant systems. Plants and fungi have established complex mutualistic relationships, and wild plants are almost always colonized by endophytic, parasitic and mycorrhizal fungi (Rodriguez et al. 2009; Patkar and Naqvi 2017). Fungi produce a variety of bioactive compounds that play an important role in the physiological activities of the host plant, influencing the growth of the hosts. This can even lead to an increased tolerance to abiotic and biotic stresses of the plants (Pineda et al. 2010). Many studies have shown that fungi enhance plant growth through the solubilization of insoluble minerals in soil and secretion of plant growth regulators (Bilal et al. 2018; Chanclud and Morel 2016; Júnior et al. 2017; Khan et al. 2012). Growth promotion by plant growth regulators or phytohormones production, are signal molecules acting as chemical messengers and play a fundamental role in plants. Plant growth hormones produced by symbiotic fungi may greatly influence processes including 
Table 11 Different products containing mycorrhizal fungi

\begin{tabular}{|c|c|c|c|}
\hline Product & Mycorrhizae & Advantages & Use for \\
\hline Soluble Mycorrhizae $^{a}$ & $\begin{array}{l}\text { Seven strains of endo-mycorrhizae } \\
\text { fungi and five strains ecto- } \\
\text { mycorrhizae fungi }\end{array}$ & $\begin{array}{l}\text { Enhance seed germination and transplants growth } \\
\text { and new root development }\end{array}$ & $\begin{array}{l}\text { Deep root feeding, } \\
\text { transplanting plants, } \\
\text { shrubs and nurseries }\end{array}$ \\
\hline $\begin{array}{l}\text { Soluble root growth } \\
\text { enhancer } \\
(\text { Oregonism XL) }\end{array}$ & $\begin{array}{l}\text { Six strains of endotrophic and six } \\
\text { strains of ectotrophic mycorrhizae } \\
\text { species }\end{array}$ & $\begin{array}{l}\text { Enhance nutrient and water uptake, encourage } \\
\text { vigorous root systems and help plants resist } \\
\text { stress and disease }\end{array}$ & $\begin{array}{l}\text { Effective in soil and } \\
\text { hydroponics, fruits and } \\
\text { flowering plants }\end{array}$ \\
\hline $\begin{array}{l}\text { MycoStim (soil } \\
\text { inoculant) }^{\mathrm{a}}\end{array}$ & $\begin{array}{l}\text { Eight strains of mycorrhizae and two } \\
\text { species of Trichoderma }\end{array}$ & $\begin{array}{l}\text { Enhance growth and nutrient uptake, stress } \\
\text { resistance, improve plant quality, yields and } \\
\text { vigor }\end{array}$ & Flowers and vegetables \\
\hline $\begin{array}{l}\text { Root rally with } \\
\text { mycorrhizae }\end{array}$ & $\begin{array}{l}\text { Bio-nutrients and inoculated with } \\
\text { mycorrhizae spores }\end{array}$ & $\begin{array}{l}\text { Improve stress tolerance, encourage root growth } \\
\text { and increase water and nutrient absorption }\end{array}$ & $\begin{array}{l}\text { Trees, shrubs, flowers, } \\
\text { fruits and vegetables. }\end{array}$ \\
\hline $\begin{array}{l}\text { Natural biological } \\
\text { inoculant (soluble } \\
\text { powder) }\end{array}$ & $\begin{array}{l}\text { Nine mycorrhizal species, } 15 \\
\text { beneficial bacteria and two species } \\
\text { of Trichoderma }\end{array}$ & $\begin{array}{l}\text { Improve nutrient and water uptake for enhanced } \\
\text { plant performance }\end{array}$ & Flowers and vegetables \\
\hline
\end{tabular}

${ }^{a}$ https://www.planetnatural.com/product/soluble-mycorrhizae/

${ }^{b}$ https://www.planetnatural.com/product/roots-organics-oregonism-xl/

${ }^{c}$ https://www.planetnatural.com/product/root-rally/

${ }^{\mathrm{d}}$ https://www.planetnatural.com/product/myco-madness/

seed germination, root initiation, stem and leaf growth, phototropism, flowering and fruit growth (Khan et al. 2015b; Petracek et al. 2003). On the other hand, many fungal pathogens produce phytohormones during host invasion and colonization, which are mainly involved in plant defense response (Kazan and Lyons 2014; Spence et al. 2015).

The use of plant growth hormones in agriculture and horticulture has been growing significantly. One of the best studied plant growth regulators is indole-3-acetic acid (IAA), a principal auxin involved in apical dominance, root initiation, cell division and cell enlargement (Benjamins and Scheres 2008; Vessey 2003). Several fungal strains belonging to various families of Ascomycota, Basidiomycota and Mucoromycota have been reported to produce IAA (Chandra et al. 2018; Hasan 2002; Waqas et al. 2012). Recently, Numponsak et al. (2018) reported that the IAA containing crude extract of a strain of Colletotrichum fructicola increased coleoptile elongation of rice, corn and rye, in a similar manner as the commercial IAA standard. Moreover, when the spore suspension of $C$. fructicola was applied in rice seedlings, it accelerated the growth of seedlings and enhanced their biomass and chlorophyll content (Fig. 22).

Gibberellins were first discovered in the culture filtrate of the pathogen "Gibberella" (now classified in Fusarium) fujikuroi, which causes disease in rice plants (Hedden and Sponsel 2015). This hormone is used to accelerate the processes of seed germination, stem elongation, leaf expansion, flower initiation and fruit development (Yamaguchi 2008). Gibberellic acid can induce bolting and

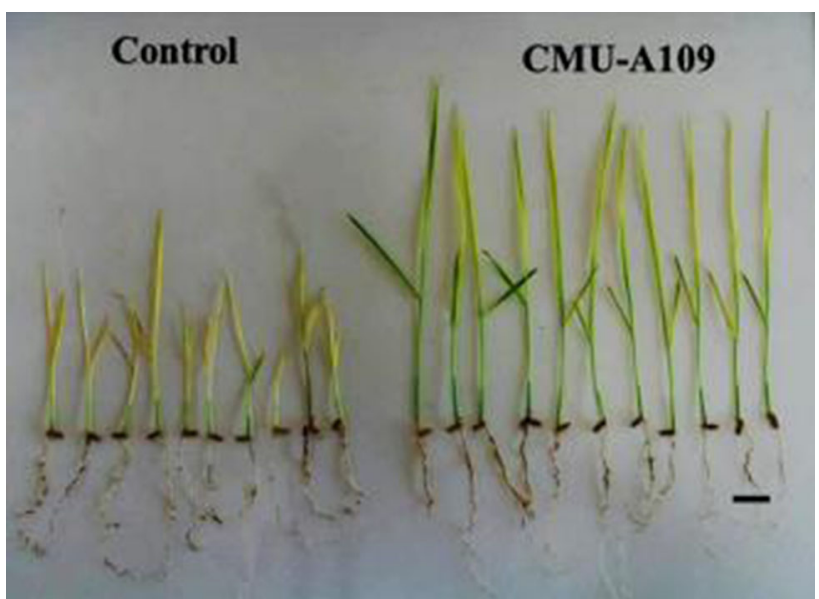

Fig. 22 Influence of a spore suspention of Colletotrichum fructicola CMU-A109 on the growth of rice seedlings after 10 days (right) against the untreated control (left). Bar $2 \mathrm{~cm}$

flowering in rosette species and rescue dwarf mutants of maize and peas (Hedden and Sponsel 2015).

Cytokinins (CKs) were reportedly found in pathogenic fungi such as Leptosphaeria maculans, Magnaporthe oryzae, and in mycorrhizal fungi (Crafts and Miller 1974; Chanclud et al. 2016; Trdá et al. 2017). This hormone plays a significant role in plant physiological processes including apical dominance, cell division, leaf senescence, chloroplast biogenesis, vascular and shoot differentiation, programmed cell-death and anthocyanin production (Fahad et al. 2014).

Abscisic acid (ABA) is another important plant growth hormone, which was discovered as secondary metabolite of 
various fungi, including species of Aspergillus, Botrytis, Cercospora, Penicillium and Rhizopus (Shi et al. 2017). Abscisic acid plays a significant role in plant responses and adaptations to various environmental stresses, thus increasing crop yields (Devinar et al. 2013; Narusaka et al. 2003). Moreover, some fungi are able to produce ethylene (ET), salicylic acid (SA) and jasmonic acid (JA) hormones that regulate plant defense against pathogens as well as plant growth and development. Trichoderma species have been reported to simultaneously induce the ET, SA and JA pathways following pathogen attacks in Arabidopsis thaliana, grape, tomato and melon (Jogaiah et al. 2018).

The production of plant hormones by fungi depends on their growth conditions, such as temperature, $\mathrm{pH}$, incubation period, growth dynamics and internal physiology (Bilal et al. 2018; Khan et al. 2012). Optimization using statistical approach is necessary to improve yield for production of phytohormones and other bioactive metabolites in the industry level (Albermann et al. 2013). Although plant growth regulators are widely found in plants, fungi and bacteria, they are being produced by chemical synthesis at the commercial scale. The high cost and low productivity of the proceesses available to access microbial-derived plant hormones limit their extensive application and thus restrict the development of the industry (Shi et al. 2017). Currently, ABA is produced with high yielding strains of $B$. cinerea and has been available commercially since 2009 (Rademacher 2015). Furthermore, in case of GAs production, Fusarium (Giberella) fujikuroi produces relatively high titers and therefore it is the principally utilized strain for industrial production. The possibility of the chemical synthesis of GAs was studied, and it was found that the compounds were too complex and the process too expensive to be a commercially viable alternative (Mander 2003). In order to increase the GAs titer, the mutagenesis of Fusarium fujikuroi and the use of cell immobilization together with extractive fermentation techniques were successfully applied in the production of GAs (Eleazar et al. 2000; Lale et al. 2006). In general, 60\% to $90 \%$ of the total applied fertilizer was lost and the remaining $10 \%$ to $40 \%$ were taken up by plants. Only Trichoderma spp. and mycorrhizal fungi are commercially produced and applied in crop production.

Crop production not only faces the challenges of climate change and the diseases that affect them, but also increases in food demands due to the burgeoning global population. Biotic and abiotic stresses are also important limitations on global crop productivity. Hence plant hormones are playing an increasingly significant sole in the horticulture and agricultural field. However, the low efficiency of fungal fermentation processes continues to preclude the cost reductions necessary for industrial-scale production. Thus, the study of fungal genome sequencing may help to shed light on the presence of the hormonal biosynthesis pathways. The molecular biology and transcriptomic analyses of fungal-derived plant hormones may provide more details related to the effects of phytohormones on plants, and ultimately effect the increased productivity of these hormones.

\section{Mitigating abiotic stress in plants: the endophyte method}

Abiotic stresses such as drought, salinity, extreme temperatures and oxidative stresses adversely affect plant growth and productivity (Fahad et al. 2017). Many of these stresses, either individually or in combinations, take a heavy toll on agricultural productivity in most parts of the semi-arid tropics (McCartney and Lefsrud 2018). In recent decades, the effects of such abiotic stresses have been further exacerbated by unprecedented changes in climate (Fedoroff et al. 2010). Conventional crop improvement approaches to render plants tolerant to abiotic stresses and resilient to climate change have been met with limited success, primarily due to the combination of the stressors and the multitude of plant traits involved in determining tolerance. A more recent and exciting approach has emerged from the use of endophytic fungi to alter plant responses and adaptation to abiotic stresses.

Endophytic fungi coexist with plants without causing any apparent disease symptoms. Several studies have demonstrated the role of endophytic fungi in enhancing plant fitness, within both, normal and stressful environments (Abdelaziz et al. 2017). The rationale for an endophyte-based adaptation rests on the fact that the endophytes adapt to environmental adversities faster than their host plants, and often are also able to collaterally share such adaptations with their respective host plants (Suryanarayanan et al. 2017). Careful exploitation of endophytic fungi could offer a strategic approach to alleviate stress effects in non-host plants, such as crop species (Rodriguez et al. 2008). Here, we briefly review studies that have explored the use of endophytes in modulating plant responses to abiotic stresses and discuss how these could potentially be used to mitigate abiotic stressors in crop plants.

Abiotic stresses such as drought, salinity, extreme temperatures and oxidative stresses adversely affect plant growth and productivity (Fahad et al. 2017). Many of these stresses, either individually or in combinations, take a heavy toll on agricultural productivity in most parts of the semi-arid tropics (McCartney and Lefsrud 2018). In recent decades, the effects of such abiotic stresses have been further exacerbated by unprecedented changes in climate (Fedoroff et al. 2010). Conventional crop improvement approaches to render plants tolerant to abiotic stresses and 
resilient to climate change have been met with limited success, primarily due to the combination of the stressors and the multitude of plant traits involved in determining tolerance. A more recent and exciting approach has emerged from the use of endophytic fungi to alter plant responses and adaptation to abiotic stresses.

Scores of studies have examined the role of endophytes in enabling plant adaptation to abiotic stresses. One of the most extensively studied fungi is Piriformospora, a root endophyte, isolated from woody shrubs of the Thar Desert, India (Varma et al. 1999). Recently, following a taxonomic revision, the fungus has been renamed Serendipita indica (Weiß et al. 2016). The fungus readily colonizes a wide array of plants and imparts tolerance to abiotic stresses such as drought, salinity, osmotic and heavy metals (Hosseini et al. 2017). Aside from conferring adaptation to abiotic stresses, $S$. indica colonization of soybean plants was shown to improve plant growth and also nutrient acquisition (Bajaj et al. 2018). Under salinity stress, maize plants colonised by $S$. indica produced higher biomass and maintained higher shoot potassium ion content compared to un-inoculated plants (Yun et al. 2018). Using rice as a model system, studies have shown that endophytic fungi from salt-adapted plants enhance growth and yield of salt sensitive rice varieties under salinity stress when compared to plants not colonized by such fungi (Redman et al. 2011; Yuan et al. 2016). Sangamesh et al. (2017) demonstrated the ability of endophytes isolated from plants adapted to deserts to not only successfully colonize non-host plants such as rice, but also to impart thermo-tolerance to them under laboratory conditions. In a meta-analysis conducted on 94 strains of endophytes and 42 host plants, Rho et al. (2018) reported that, overall, endophyte colonization led to effective mitigation of drought and salinity stress as well as nitrogen deficiency. The study also showed the ability of endophytes to readily colonize and establish plant-endophyte relationships. The existing evidences suggest that endophytes from stress-adapted plants could be transferred across plants of varied phylogenetic affiliations.

The immediate physiological and molecular basis of plant-endophyte interactions, including how endophytes from plants adapted to extreme habitats are able to confer resistance to non-host plants, are only beginning to be addressed. A cyclophilin A-like protein (PiCypA) obtained from Serendipita indica has been implicated in the ability of the fungus to impart salinity tolerance. Transgenic tobacco plants overexpressing PiCypA exhibited higher salt stress tolerance compared to wild type plants (Trivedi et al. 2013). A salt responsive gene, PiHOG 1 from $S$. indica, was shown to impart osmotic and salt stress tolerance to rice plants when compared to mutants in-vitro. Treatment of $S$. indica knock down, KD-PiHOG1 resulted in decreased colonization and reduced tolerance to salt stress (Jogawat et al. 2016). Arabidopsis plants inoculated with S. indica exhibited ion homeostasis under salt stress. These plants had higher transcript levels of high affinity potassium transporters, $H K T 1$ and inward rectifying $\mathrm{K}^{+}$channels, $K A T 1$ and $K A T 2$, compared to plants without the fungus (Abdelaziz et al. 2017). Under both normal and low phosphorous conditions, S. indica activated signaling, transport, metabolic and developmental programs in roots of Arabidopsis (Bakshi et al. 2017). The fungus was also shown to cause global reprogramming of host metabolic compounds and metabolic pathways in Chinese cabbage (Hua et al. 2017).

It is evident from existing studies that endophytes offer a promising option to mitigate abiotic stresses in crop plants. The single most important advantage of this approach is that it offers a non-genetic-invasive method to alter plant phenotype, when compared to conventional and molecular breeding approaches (Gopal and Gupta 2016); furthermore, it is rapid and cost-effective. Several initiatives have been launched to harness the power of endophytes, including BioEnsure, a product approved for use by the US Food and Drug Administration, and Rootonic, a mixture of S. indica biomass and magnesium sulphate (Jones 2013; Shrivastava and Varma 2014). The product Bioensure was able to stabilize yields of maize under drought, increase seed germination under freezing stress several-fold, and improve the water use efficiency of plants (Jones 2013). Rootonic treatment to seeds provided multifarious benefits to the plants, under both normal and stressful conditions (Shrivastava and Varma 2014). Rapid methods of deployment of select endophytes, either through seed priming or through foliar or floral dip methods, could offer a quick and safe agronomic strategy to mitigate abiotic stress in plants (Fig. 23). This approach also resonates in its application to major crop plants that may have lost many of their native endophytes during the process of domestication. For these plants, the endophyte-enrichment approach could in fact be a process of returning to their roots (Oyserman et al. 2018).

\section{Food and beverages from fungi}

Food and beverages from fungi is a multi-million dollar business, and in particular in Asia, much of the development has concentrated on mushrooms because of their nutritious qualities and medicinal importance. In this section, we discuss the rapid development of the mushroom industry in Asia, outline the mushrooms used in Traditional Chinese Medicine, highlight one of the most revered medicinal mushrooms (Agaricus subrufescens), and introduce various food products from fungi. 
Fig. 23 A schematic illustration of the events depicting the use of endophytic fungus in mitigating abiotic (salinity) stress in crop plants. Clockwise: A saline adapted plant, Prosopis juliflora (1); isolation of endophytes (2); evaluation of endophytic fungus for salinity tolerance (3); priming seeds of non-host, salt sensitive paddy, with saline tolerant endophyte (4); growth of endophyteenriched and non-enriched seedlings under saline stress (5); growth of enriched and nonenriched paddy plants under saline stress (6); transgenerational effect of paddy plants under saline stress (7). The model could be followed for other abiotic stresses as well (all photographs: School of Ecology and Conservation, University of Agricultural Sciences, GKVK, Bangalore)

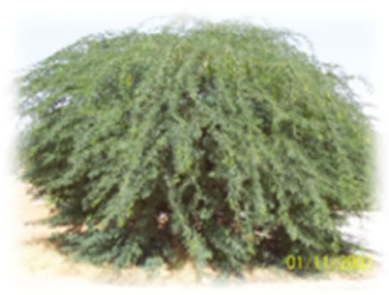

1.Saline habitat adapted plant

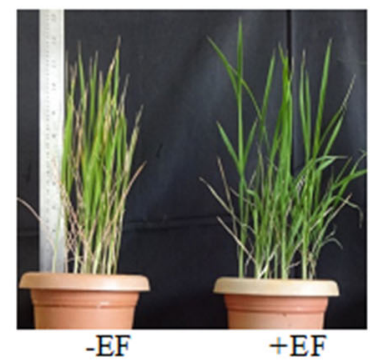

7. Trans-generational effect of seed priming under $\mathrm{NaCl}$ stress
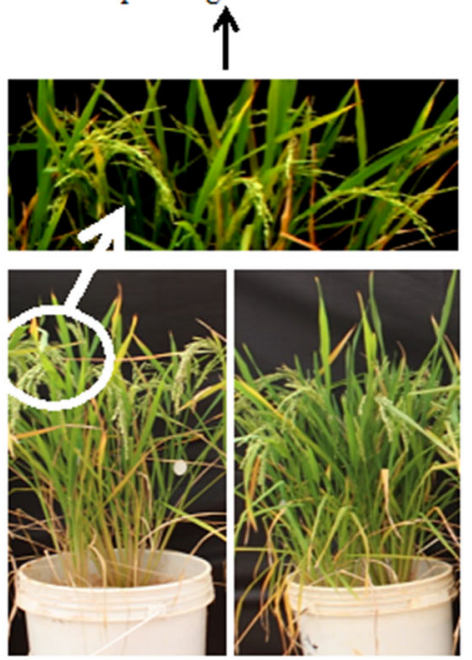

$-E F$

6.Growth of paddy plant enriched with saline tolerant EF under salt stress
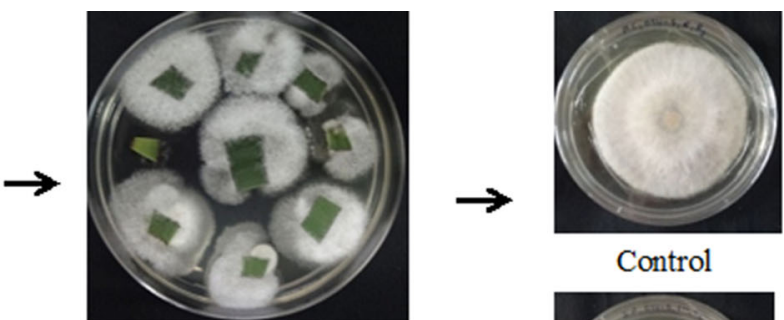

Control

2. Isolation of saline habitat adapted endophytic fungi (EF)

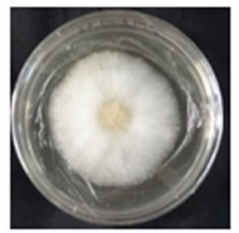

$1 \mathrm{M} \mathrm{NaCl}$

3. Evaluation of salinity tolerance
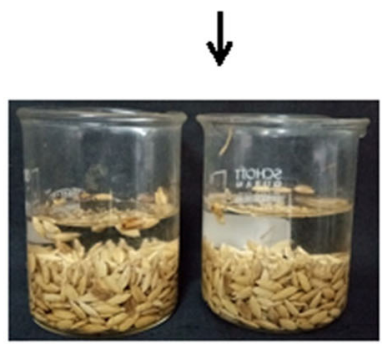

$-E F$

$+\mathrm{EF}$

4. Priming of non-host (paddy) seeds with saline tolerant EF

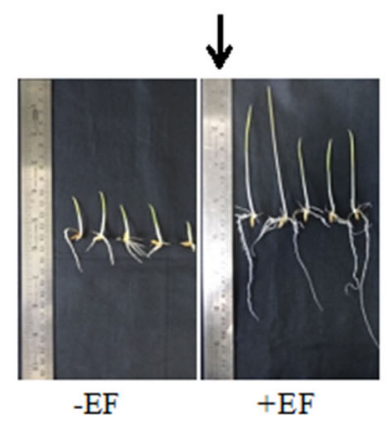

5. Growth of paddy seedlings enriched with saline tolerant $\mathrm{EF}$ under $\mathrm{NaCl}$ stress

\section{Growing mushrooms in composts}

Some mushrooms require a composting process for industrial production, as in Agaricus species. The methods for growing Agaricus species differ from those used in growing many other mushrooms, which are grown in sawdust mixtures in bags. Agaricus is a secondary decomposer. The preparation of the substrate for growing Agaricus species is a process known as composting. Within this process, bacteria and other fungi break down the raw materials in the compost mixture, which allows Agaricus species to grow on the fermented substrate (Llarena-
Hernández et al. 2011). The first formal mention of mushroom cultivation based on the composting method was in 1650 in Paris. However, cultivated mushrooms were reported to be different in appearance to those harvested from the field and not as good to eat. In 1707, a French botanist reported that mushrooms were produced from horse manure covered by soil. The first record of commercial cultivation was in 1780 by a French gardener, while mushroom growing was introduced to North America after the Civil War (Beyer 2003). In 1800, the first cultivation of Agaricus in caves was achieved in Paris. 
In Europe and Brazil, the basic raw material used in composting is wheat straw. hay, corncobs, oat, barley, sugarcane bagasse, rye grass, rice straw, several other grasses or spent mushroom waste can act as alternative substrates (Royse and Chalupa 2009; Stamets and Chilton 1983; Mendonça et al. 2005; Llarena-Hernández et al. 2011, 2014; Grimm and Wösten 2018). Supplements such as soybean meal and cereal bran mixed with minerals or vitamins are added for cultivation of Agaricus and Pleurotus mushrooms (Carrasco et al. 2018). There are normally two phases in composting. Phase $\mathrm{I}$ is called composting, which involves mixing the piled-up ingredients. Supplements are added to the watered stack and this is incubated for 3-15 days. The temperature inside the compost stack can reach $70-80{ }^{\circ} \mathrm{C}$, and thermophilic microbes in the compost utilize carbohydrates and free ammonia. After fermentation, the compost is pasteurized at $60{ }^{\circ} \mathrm{C}$. Phase II is called conditioning, at which point the temperature is maintained at $45-60{ }^{\circ} \mathrm{C}$ for the culturing of good microbes, and to decrease the ammonium level of the compost (Mendonça et al. 2005; Stamets and Chilton 1983; Grimm and Wösten 2018).

In Asia composting has been modified as wheat straw and horse manure are not readily available. Chopped rice straw is used as the main substrate. The rice straw is supplemented with rice bran, urea, ammonium phosphate, calcium sulfate to increase nutrients. Gypsum and calcium carbonate are added for buffering the $\mathrm{pH}$. The process occurs outdoors. For pasteurization, the compost is heated to $55-60{ }^{\circ} \mathrm{C}$ for $3-6 \mathrm{~h}$ with steam (Royal project's method, unpublished data). When the compost is generally used and inoculated with spawn. After the mycelium runs through the compost the casing is covered to stimulate fruit-body production (Llarena-Hernández et al. 2013). Different mixtures are used for casing inclduding soil: wood, charcoal: calcitic lime (Zied et al. 2010), soil: sand mixed with peat (Mendonça et al. 2005) limestone: peat: thin sand (Llarena-Hernández et al. 2014) after casing. Then, the mycelium grows within the casing layer in about 1520 days depending on environmental factors, Agaricus requires around 20-30 days (Mendonça et al. 2005).

Methods of cultivation have been developed that use local agricultural waste as the main substrate, instead of wheat or rice straw. Specific media need to be established for the production of fruit-bodies depending on the species of mushroom.

\section{Mushrooms grown in compost}

Agaricus bisporus (Button mushroom)

The first white cultivar of Agaricus bisporus was found in 1926 by Lewis Downing, an American farmer, who passed it on to a spawn company. Four year later, the spawn had been spread worldwide. This cultivar named "smooth white" is one of parents of the hybrid strain most cultivated in the world. The basic cultivation method for A. bisporus was established and developed by Sinden and Hauser (1950, 1953). Agaricus bisporus can be grown on standard compost based on wheat straw and horse manure, as well as other agricultural waste (Colak 2004; Carrasco et al. 2018). Peat, sand, peat and lime (1:1:1); farm yard manure and soil $(1: 1)$; tea production waste mixed with peat; and protein-rich supplements (soybean, black beans and cowpeas) can be used as a casing layer (Gülser and Pekşen 2003; Coello-Castillo et al. 2009; Gupta et al. 2018) Agaricus bisporus can also be cultivated using autoclaved non-composted substrates (Till 1962; Colmenares-Cruz et al. 2017).

Other Agaricus species

Agaricus flocculosipes was first reported by Zhao et al. (2012) and is currently only known to be found in Thailand, Mayotte Island and China. Thongklang et al. (2014a) reported the first successful cultivation of A. flocculosipes in Thailand on wheat straw and horse manure compost. The primordia of Thai A. flocculosipes occurred at day 32 after casing, and the strain produced a few fruiting bodies (Fig. 24). Agaricus subtilipes (Fig. 24) was described in Thailand by Thongklang et al. (2016). Cultivation of A. subtilipes was successful on wheat compost with fruiting bodies produced 45 days after casing. Although A. flocculosipes and A. subtilipes produced fruiting bodies, the yields were low and the fruiting period was shorter when compared to Thai strain of A. subrufescens (Fig. 24). These Thai Agaricus species are candidates for domestication and cultivation and have potential medicinal use. But first the optimal conditions for growing these mushrooms must be established, and any bioactive compounds investigated. In addition, the optimal conditions for fruiting must be established for increasing yield production. Hyde et al. (2018b) reported that 93\% of Agaricus species found in northern Thailand were new to science and, as many are edible, they have high potential for domestication.

Pleurotus ostreatus (oyster mushroom)

This mushroom is cultivated worldwide, grows easily in various substrates, and has important nutritional and medicinal properties. The oyster mushroom is a primary decomposer and can degrade many components of plant material, including hemicellulose, lignin and more (Grimm and Wösten 2018). The mushroom is produced on rubber sawdust, rubber sawdust mixed with different supplements of rice straw, rice husks or corncobs in Thailand via bag cultivation (Thongklang and Luangharn 2016). Vieira and de Andrade (2016) reported that compost was used to cultivated $P$. ostreatus in Brazil. Different substrates, 
Fig. 24 Wild Thai mushrooms cultivated in compost media. a A. subrufescens (Thongklang et al. 2014a). b Agaricus flocculosipes (Thongklang et al. 2014a), c Lepista sordida (Thongbai et al. 2017), d A. subtilipes (Thongklang et al. 2016), e Macrolepiota dolichaula (Rizal et al. 2016)

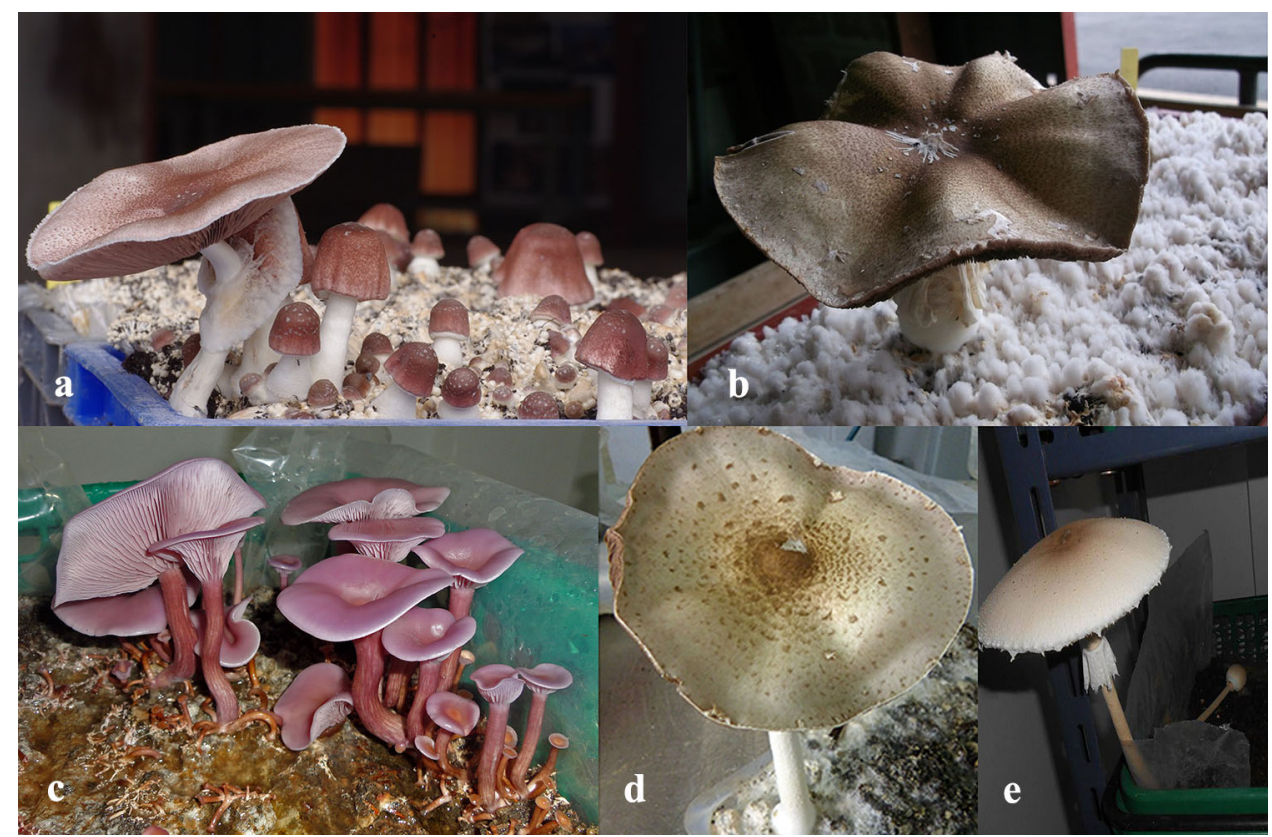

including decumbens grass, sugarcane straw, brizantha grass, wheat straw, and sugarcane bagasse, supplemented with and without wheat bran as the primary substrates, were studied and brizantha grass was found to be the optimal substrate for cultivating $P$. ostreatus. Supplementing the compost with wheat bran improved yield in all substrates tested. In addition, the conditioning of compost after 7 days for all substrates showed higher yield and biological efficiency for P. ostreatus. The composts supplemented with $20-40 \%$ composted or $20 \%$ raw two-phase olive mill waste were reported as potential substrates for Pleurotus cultivation (Zervakis et al. 2013). In addition, semi-compost made from wheat straw and sunflower husks (2:3) can be used to grow Pleurotus sp. The substrate was fermented by aerobic fermentation for $36 \mathrm{~h}$ at $65{ }^{\circ} \mathrm{C}$ followed by cooling to $25^{\circ} \mathrm{C}$ over $24 \mathrm{~h}$ (Myronycheva et al. 2017).

\section{Other genera}

Thongbai et al. (2017) used a rice straw based compost to cultivate Lepista sordida. Rice straw mixed ammonium phosphate, calcium carbonate, calcium sulfate, rice bran and urea were used as main substrates. The mycelium of L. sordida was inoculated into the pasteurized compost, which was later covered with a casing layer mixture of $15 \%$ sand with humus. Lepista sordida produced fruiting bodies after 31 days at $25{ }^{\circ} \mathrm{C}$ with $95-97 \%$ humidity after casing (Fig. 24). Macrolepiota dolichaula is a wild edible mushroom that is widely consumed in China, India and northern Thailand. This mushroom also can grow well in rice straw based compost. Mushroom production occurred at 41 days with four flushes at $25{ }^{\circ} \mathrm{C}$ with $92 \%$ relative humidity (Fig. 24, Rizal et al. 2016).

Mushrooms have long been used for consumption, medicinal purposes and functional foods; however, the recognition that they are nutritionally a very healthy food group that contains important sources of biologically active compounds of medicinal value is more recent. Mushrooms have become a hot issue, especially in Asia. Sustainable success of mushroom growing depends on research and development to establish new ideas and methods for improving cultivation. Agricultural waste can be used as substrate for growing mushrooms, resulting in lower cost as well as the productive utilization of waste.

At present, most domesticated mushroom species are international species or variants, and few local strains are grown. The price of those mushrooms is quite low and there is little potential for export. Therefore, developing novel species or strains for local cultivation has become important. New wild species or strains of mushrooms will provide high value products with nutritional and medicinal potential. However, the successful development of a newly cultivated mushroom depends on economical and biological factors, the ease of production and acceptance in the market. After establishing edibility and cultivability, the best conditions for growth must be established. Best cultivation techniques include finding the optimal growth media, $\mathrm{pH}$, temperature, $\mathrm{C}$ source, $\mathrm{N}$ source, $\mathrm{C}: \mathrm{N}$ ratio, as well as the best spawn, compost and casing. 


\section{Growing mushrooms in bags}

Wood-inhabiting mushrooms can be cultivated in any type of lignocellulosic material, such as straw, sawdust or rice hulls (Victor and Olatomiwa 2013). Pathmashini et al. (2008) showed that the cultivation of the oyster mushroom (Pleurotus ostreatus) on various sawdust types produces different fruiting yields. Sawdust is the most commonly used material for the cultivation of oyster mushrooms and is the preferred medium for commercial production (Oei 2005). Maximum biological efficiency of oyster mushroom cultivation is gained from growth on the sawdust of rubber trees (Pathmashini et al. 2008). It has also been shown that softwood sawdust such as coconut, cashew, mango and rubber are more suitable than hardwood sawdust (Custodio and Cristopher 2004). The availability of raw materials such as sawdust, rice straw, sugarcane wastes are key factors in the choice of agricultural waste for growing mushrooms (Pathmashini et al. 2008). The most commonly and easily cultivated mushrooms in South East Asian countries are oyster mushrooms (Pleurotus), ear mushrooms (Auricularia), and straw mushrooms (Volvariella), Lentinula edodes (shiitake), as well as Lentinus (e.g. Lentinus squarrosulus), Ganoderma, Macrolepiota and "Agrocybe" (i.e., Cyclocybe) spp. (Kwon and Thatithatgoon 2004).

Different types of sawdust are used as the growing medium in tropical areas, depending on the characteristics of the area and the trees available. Rubber tree sawdust is the most popular (Kwon and Thatithatgoon 2004; Klomklung et al. 2012; Nguyen 2004), followed by Acacia auriculiformis (Tapingkae 2005), Mangifera indica (Tong and Rajendra 1992) and Tamarindus indica. For every $1 \mathrm{~kg}$ saw dust bag, additions are made of $10 \mathrm{~g}$ of calcium carbonate, $50 \mathrm{~g}$ of ricebran, $10 \mathrm{~g}$ of pumice, $10 \mathrm{ml}$ of molasses, $10 \mathrm{~g}$ of flour and $10 \mathrm{~g}$ of brewer's waste. These components are then mixed with water to obtain a water content of $65-70 \%$. Each $800 \mathrm{~g}$ of substrate is then tightly packed in a $25.8 \mathrm{~cm}$ polypropylene bag and capped with a plastic ring or bottle neck, leaving space to later inoculate with mycelium (Kwon and Thatithatgoon 2004; Stamets 2000; Klomklung et al. 2012; Yamamaka 1997). Each sawdust bag is sealed with a cotton wool plug, covered with newspaper, and tied with a rubber band. The sawdust bags are sterilized at $121{ }^{\circ} \mathrm{C}$ for $15 \mathrm{~min}$ or at $90-100{ }^{\circ} \mathrm{C}$ for $3 \mathrm{~h}$. After the temperature drops to $25^{\circ} \mathrm{C}$, the bags are inoculated with spawn (Fig. 25c) that comprises $10 \%$ of the weight of the sawdust bag. Sawdust bags are kept at room temperature $\left(25{ }^{\circ} \mathrm{C}\right)$ at $70-80 \%$ humidity to produce fruiting bodies (Klomklung et al. 2012).

When new wild mushrooms are introduced to the market, it is important to conduct fruiting tests. Depending on the type of mushroom, a choice can be made between compost or sawdust media. As a rule of thumb, for woodinhabiting mushrooms (e.g. Lentinula, Auricularia) it is better to use sawdust media in bags (Fig. 25a, b), while for soil-inhabiting mushrooms (e.g. Agaricus, Macrolepiota) it is better to use straw compost.

For wood-inhabiting mushrooms, protocols adapted from Klomklung et al. (2012) are followed. The surface of sawdust growing bags is inoculated with spawn. The bags are kept in a dark incubation room at the optimum temperature and relative humidity of the particular mushroom. Bags are opened when the mycelium has completely colonized the substrate. The surface of the substrate is scraped slightly with a sterile teaspoon to remove the thin whitish

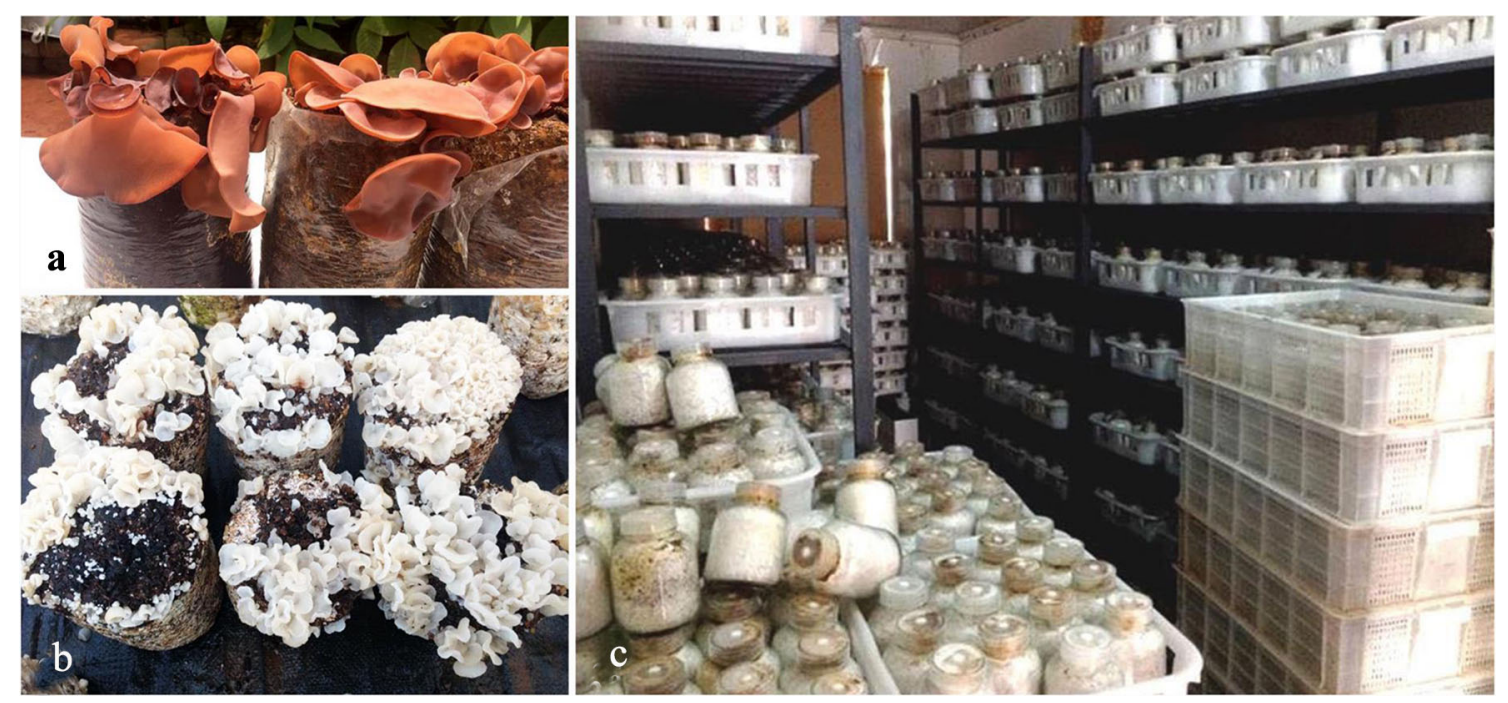

Fig. 25 a Auricularia thailandica growing on sawdust substrate bags; b Auricularia cornea white variety growing on sawdust substrate bags; c mushroom spawn bottles 


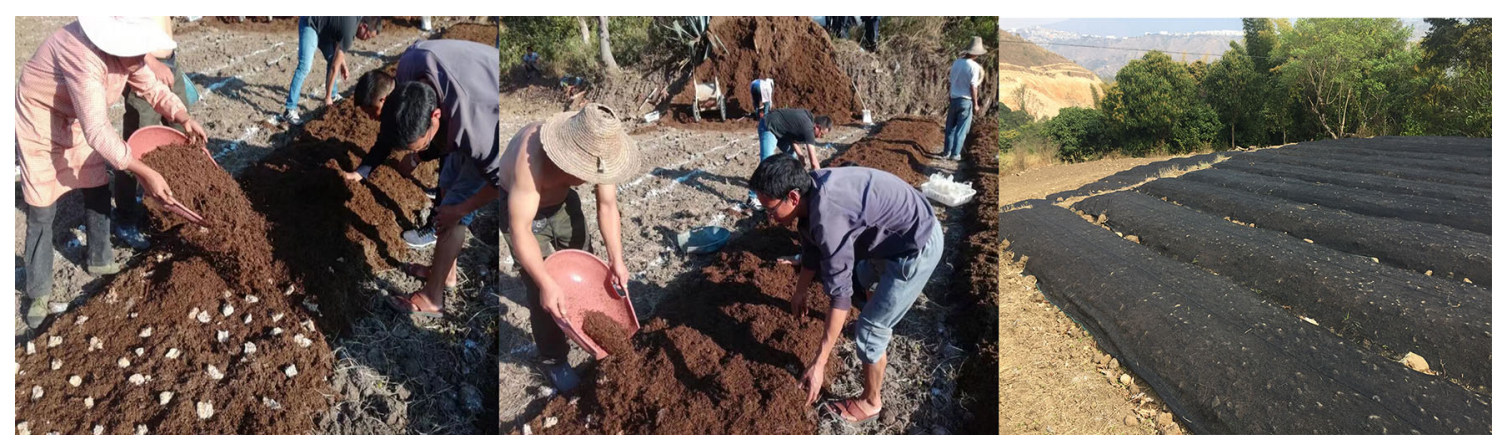

Fig. 26 Field preparation for cultivation of Stropharia rugosoannulata in Honghe County, China

Table 12 Examples of mushroom species suitable for field cultivation, including soil characteristics, substrates, and climatic requirements for the listed species

\begin{tabular}{|c|c|c|c|c|c|c|}
\hline Species & Soil type & $\begin{array}{l}\text { Soil } \\
\text { pH }\end{array}$ & Substrate & $\begin{array}{l}\text { Temperature } \\
\left({ }^{\circ} \mathrm{C}\right)\end{array}$ & $\begin{array}{l}\text { Humidity } \\
(\%)\end{array}$ & References \\
\hline Coprinus comatus & Sandy & 7 & Cottonseed meal & $16-22$ & $85-95$ & Chen (2000) \\
\hline $\begin{array}{l}\text { Dictyophora } \\
\text { indusiata }\end{array}$ & Loam & $6.5-7$ & $\begin{array}{l}\text { Bamboo litter, straw, } \\
\text { sawdust }\end{array}$ & $22-30$ & $60-65$ & Chen $(2000)^{\mathrm{a}}$ \\
\hline Ganoderma sp. & Sandy & $\begin{array}{r}4.2- \\
5.3\end{array}$ & Sawdust & $23-34$ & $80-90$ & $\begin{array}{l}\text { Mayzumi et al. (1997), Cha and } \\
\text { Yoo (1997) }\end{array}$ \\
\hline Lentinula edodes & Clay loam & $4.5-6$ & $\begin{array}{l}\text { Sawdust, wheat/rice } \\
\text { bran }\end{array}$ & $5-20$ & $80-90$ & a \\
\hline Morchella spp. & Sandy loam & $7-7.5$ & $\begin{array}{l}\text { Sawdust, wheat bran, } \\
\text { humus }\end{array}$ & $<20$ & $50-70$ & Liu et al. $(2018 a)^{a}$ \\
\hline $\begin{array}{l}\text { Oudemansiella } \\
\text { radicata }\end{array}$ & Clay loam & $\begin{array}{r}6.5- \\
7.2\end{array}$ & Wood chips/corn cobs & $20-30$ & $60-80$ & Hao et al. (2016) \\
\hline $\begin{array}{l}\text { Phlebopus } \\
\text { portentosus }\end{array}$ & $\begin{array}{l}\text { Clay loam, } \\
\text { humus }\end{array}$ & $4-6$ & Sawdust & $27-37$ & $55-80$ & $\begin{array}{l}\text { Ji et al. (2011), Kumla et al. } \\
\quad(2011,2015)^{\mathrm{a}}\end{array}$ \\
\hline Polyporus umbellatus & $\begin{array}{l}\text { Sandy loam, } \\
\text { humus }\end{array}$ & $5.5-7$ & Leaves, humus, & $18-24$ & $60-80$ & Bandara et al. $(2015)^{\mathrm{a}}$ \\
\hline $\begin{array}{l}\text { Stropharia } \\
\quad \text { rugosoannulata }\end{array}$ & $\begin{array}{l}\text { Humus rich } \\
\text { sandy }\end{array}$ & $5-6$ & Wood chips/rice straw & $22-28$ & $70-75$ & a \\
\hline Volvariella volvacea & Clay & $7.5-8$ & Rice straw & $22-40$ & 90 & $\mathrm{a}$ \\
\hline
\end{tabular}

${ }^{\mathrm{a}}$ Rows include unpublished findings/data

mycelia. The substrate bags are then placed on a shelf and covered with black cloth to allow appropriate ventilation. They are maintained in a growing house at $80-85 \%$ relative humidity, and sprayed daily with water until pin heads appear and eventually develop into fruiting bodies. The fruiting bodies are manually harvested, counted and weighed (Klomklung et al. 2012).

\section{Growing mushrooms in the field}

With the increase in consumer awareness and subsequent demand for cultivated mushrooms, there now exists a need for alternative, cost-effective strategies of mushroom cultivation. The global mushroom industry is forecast to grow from a value of about $\$ 35$ billion USD in 2015 to nearly $\$ 60$ billion USD in 2021, thus clarifying the need for a diverse range of production styles in order to develop the industry in a sustainable manner (Research and Markets 2018). Alternatives to more traditional high-volume production techniques include the inoculation of logs, cultivation in forest understories, and use of managed forests, as well as field-grown mushrooms.

Field-grown mushrooms offer an effective use of space, allowing for the production of mushrooms in agricultural fields, alongside crops, or between cropping cycles (Fig. 26). Offering additional income to farmers, this practice has become popular with rural development programs (Zhang et al. 2014a, b; Brum and Brum 2017). Additionally, field cultivation allows for improved soil systems in agricultural fields due the increased rates of nutrient cycling and provision of organic matter into the soils (Phan and Sabaratnam 2012; Zhang et al. 2012).

In this section, we outline some of the basic principles and considerations for field cultivation while listing some 
of the key species that can be successfully cultivated in a field environment (Table 12). We will limit our discussion to the cultivation of mushrooms in agricultural fields and soils, not the broader practices of outdoor cultivation using bags or inoculated logs.

There are a range of mushrooms that can be cultivated outdoors, and appropriate species can be selected according to local growing conditions (Table 12). For example, Coprinus comatus or Stropharia rugosoannulata can be grown during the autumn and winter months when temperatures are milder, and Volvariella volvacea should be grown during the warmer summer months. Consideration for soil $\mathrm{pH}$, texture and health is also critical. The $\mathrm{pH}$ and texture will partially determine which species of mushroom will be grown, and also the degree of irrigation required to maintain desired soil water levels (Table 12). Soil health is crucial to the final product, as many species of fungi can hyperaccumulate heavy metals (Cocchi et al. 2006; Turkekul et al. 2004).

After the selection of an appropriate growing area, a few basic steps are required for the preparation of the field and subsequent cultivation. After light tilling of the field, compost is applied, followed by the application of the fungal material (spawn or colonized bags), this is then covered in compost and a final layer of soil over that (a mulch of straw or shade cloth could further protect against desiccation and drying out) (Fig. 26).

There are a wide range of applications for the field cultivation of mushrooms, but most relate to the intensification of land use systems, generation of additional sources of income, and appeal to low income groups that cannot afford the costs of establishing grow houses. This method of production has become popular in many parts of Asia where climates are suitable and smallholder farmers need alternative sources of income. For example, in Cambodia, farmers have adopted the practice of spreading spawn produced from Lentinus or Volvariella species over rice straw waste in the paddies (with supplemented watering). This provides an additional harvest of mushrooms from the paddies during the off season. Another program run in Honghe County, Yunnan Province, China, assists local farmers with the cultivation of Stropharia rugosoannulata in their fields; this is a seasonal mushroom crop produced mostly in autumn and spring when the temperatures are suitable (Fig. 27, Table 12).

The nature of growing mushrooms outdoors means that the mushroom crop is subject to ambient environmental conditions, with no climate controls in place, for example, temperature and humidity fluctuate with no real mechanisms in place to control these parameters. In addition to climatic variability, outdoor cultivation exposes the mushrooms to non-sterile conditions, allowing easy access for secondary infection by slime molds, predatory fungi, and insects (Table 12).

Field cultivation of mushrooms is applicable to a wide range of habitats and conditions, depending on the desired species to be grown. By offering novel and exciting alternatives to plant-based agriculture, and providing additional income to farmers, field cultivation of mushrooms is becoming popular in programs aimed at improving the livelihoods of smallholder farmers and for incorporation into projects looking to diversify the agricultural output of a unit of land. However, growing mushrooms outdoors is not without challenges and requires careful species selection and training of practitioners before any such programs can commence.

\section{Modern mushroom production: an automated factory process}

Limited harvests during unproductive seasons and the risk of mushroom poisoning prevent wild mushrooms from entering the food market value chain in a major way. Domestication and industry production has therefore
Fig. 27 Stropharia rugosoannulata emerging from soil substrate mix and mature crop shown. Shade cloth is pulled back to reveal harvest
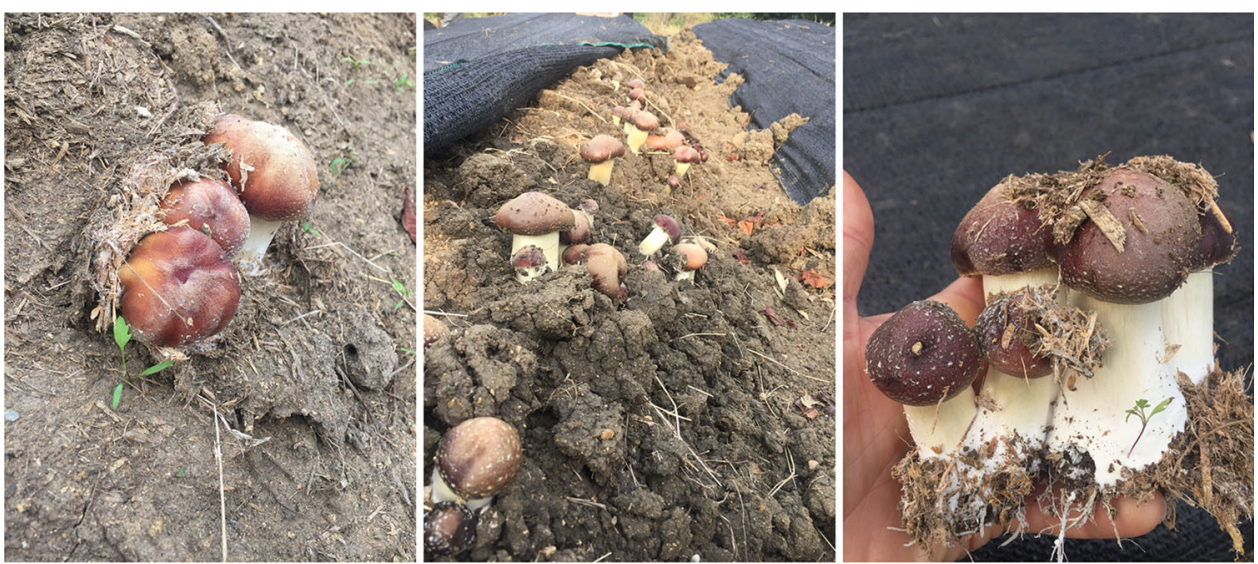

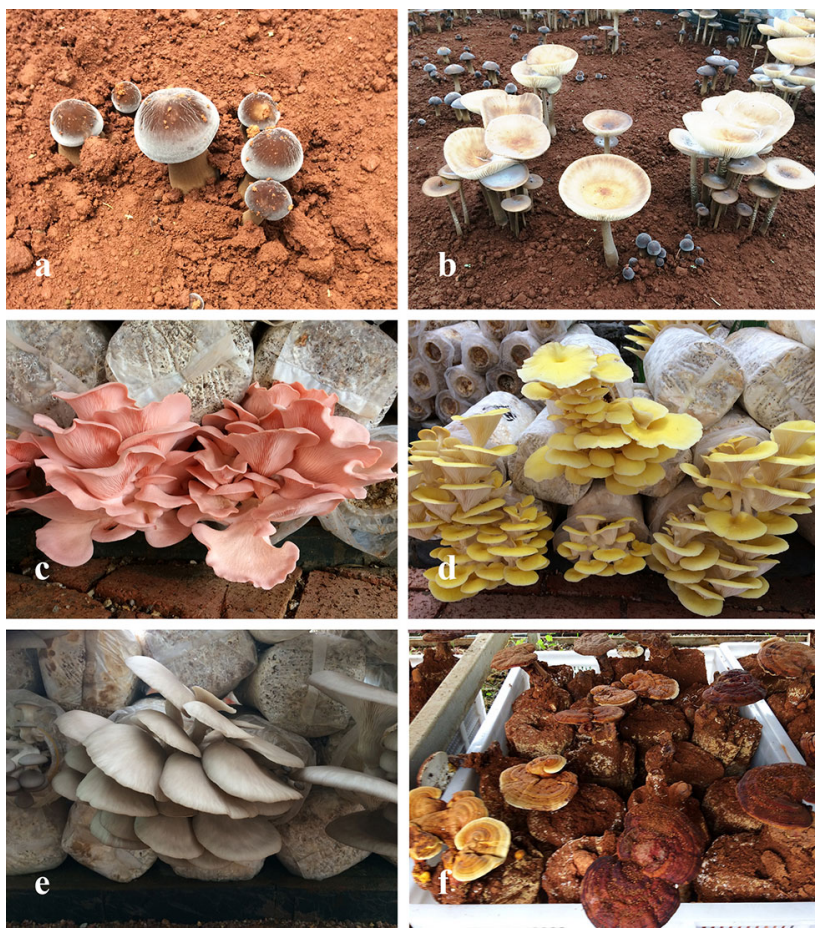

Fig. 28 Different mushroom species cultivated worldwide. a, b $\mathrm{Hy}$ menopellis $\mathrm{sp}$., c, e Pleurotus $\mathrm{sp}$. (Oyster mushrooms), f Ganoderma sp. (Lingzhi). Photo credit: T. Luangharn become essential for incorporating mushrooms into dietary diversity and nutritional security. The global production has increased from 30.2 million tons in 2010 to 48 million in 2017 (FAO). As a leading producer, China increased from 22.6 million tons in 2010 to 38.4 million in 2017 , accounting for $75 \%$ of global production (CIRI 2017). Of the 16,000 known mushroom species (Hawksworth 2012), about 7000 species have varying degrees of edibility, more than 3000 species are major edible mushrooms, and 700 are regarded as safe medicinal mushrooms (Chang and Wasser 2017).

Global demand for mushrooms has rapidly increased and thus large-scale growers have been established, with year-round marketing dominating commercial mushroom production. In China, there are six groups of mushrooms each with a production capacity of over one million tons per year (Zhang et al. 2015): 8.2 million tons for Auricularia species; 7.7 million tons for Lentinula edodes (Shiitake mushroom); 5.9 million tons for Pleurotus species; 3.4 million tons for Agaricus bisporus; 2.6 million tons for Flammulina spp; and 1.4 million tons for Pleurotus eryngii. The growth of the commercial mushroom industry depends on productivity, efficiency, and competitiveness within the entire market value chain. Most of the more recently domesticated species can recycle waste substrates

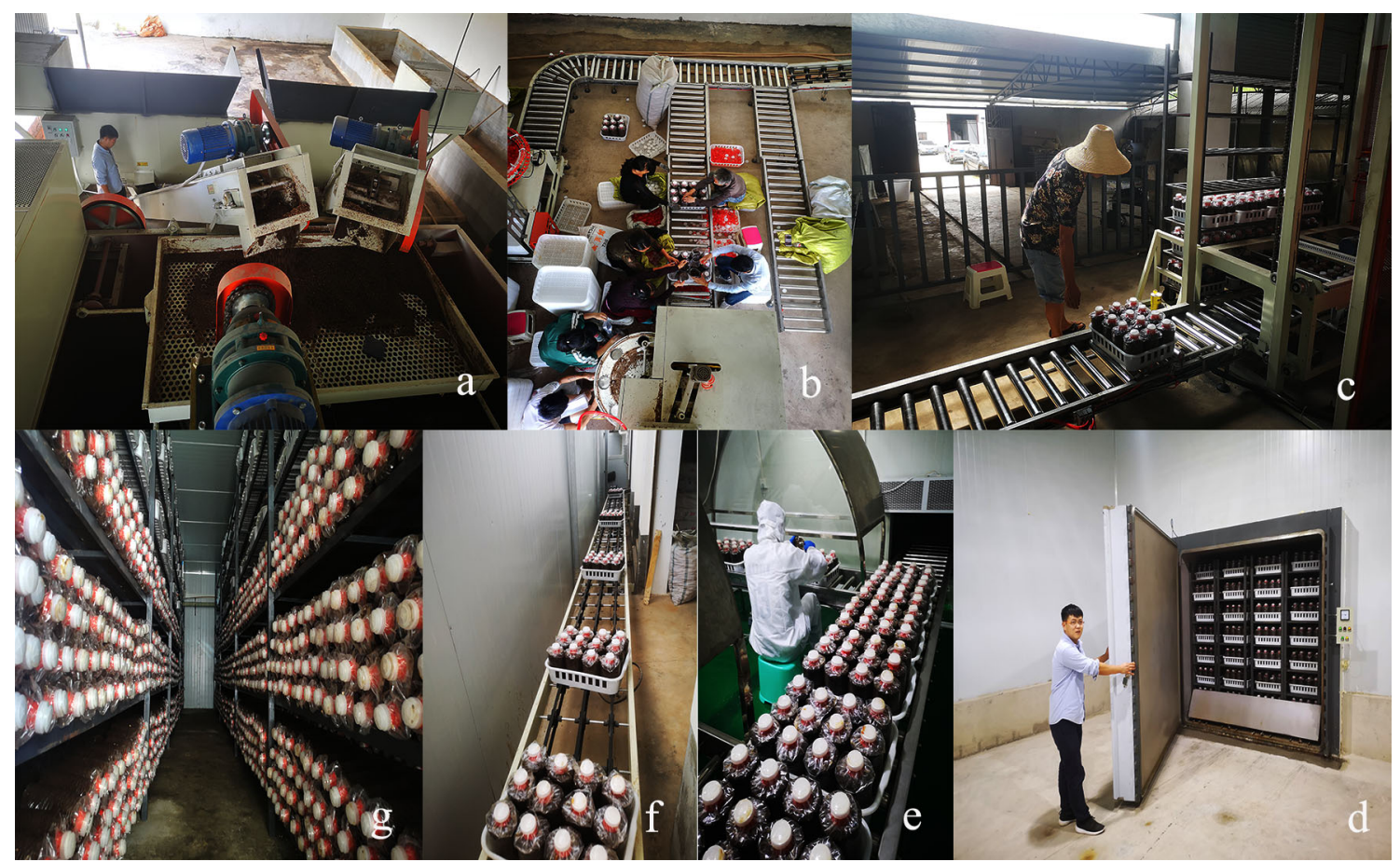

Fig. 29 Equipment for manufacturers for mushroom cultivation. a Substrate media mixer, b mushroom bag filling machine, $\mathbf{c}$ transporter and conveyors carry out substrate bags, $\mathbf{d}$ steam sterilization machine, e inoculation, $\mathbf{f}$ auto-transportation, $\mathbf{g}$ incubation. Photo credit: F. Huang 


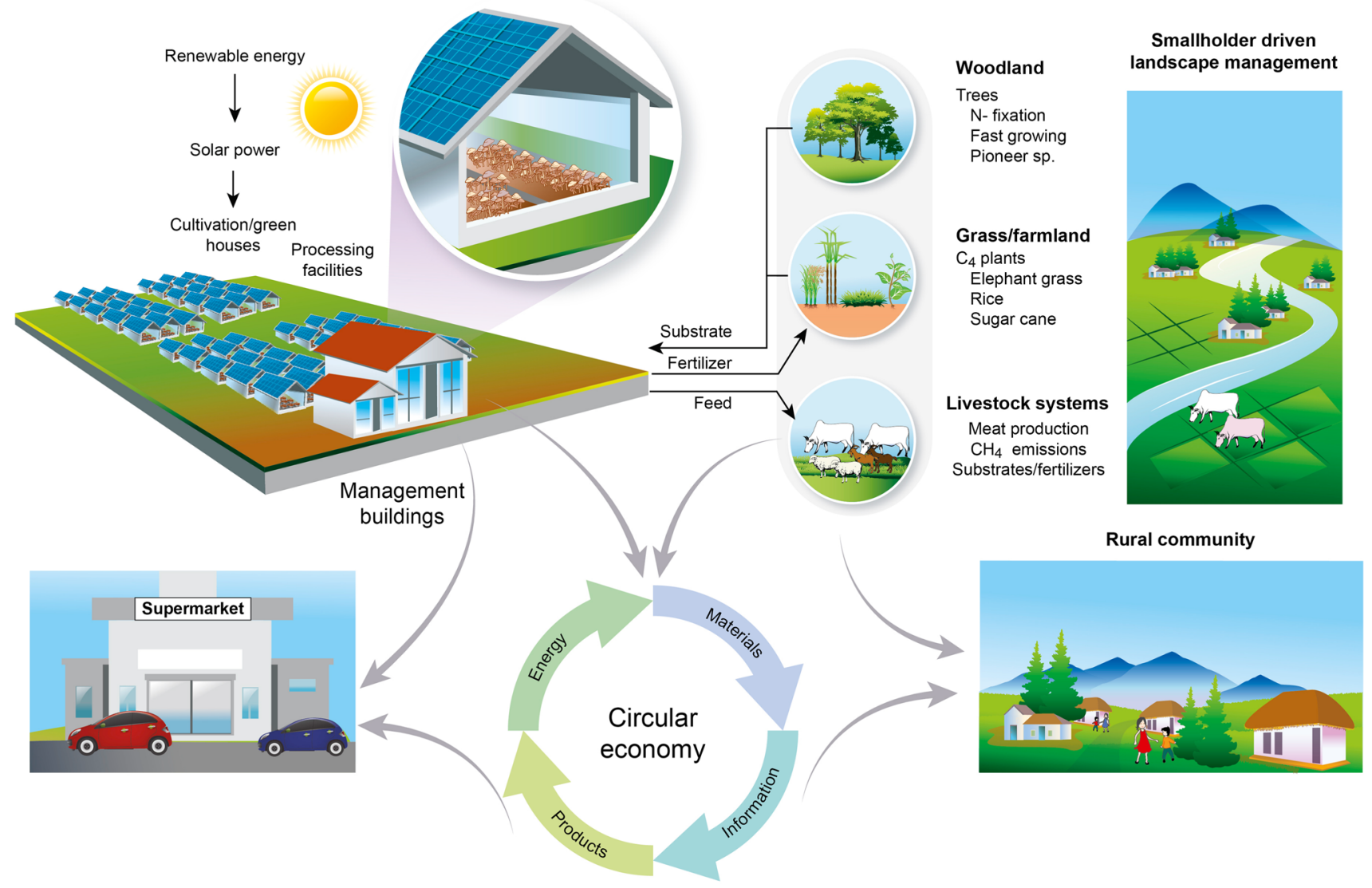

Fig. 30 Mushroom industry zone with controlled environment for rural employment and rural development

and therefore suit smallholders in agroecosystems (Dai et al. 2010b) (Fig. 28).

In the last decade, there have been remarkable advances in mushroom growing technology. Not only have strains been improved, but the whole mushroom production process has changed from manual to automated systems. The mixing of substrates, filling of bags, growing of liquid spawn, inoculation of bags, moving of bags, temperature and humidity control, and packing are all automated processes (Fig. 29).

Reliable substrate sources and machinery are two of the factors that determine the scale of operation and productivity. Substrates can be harvested from nearby forests through sustainable forest management, from recycled agricultural residue, sugarcane factories, livestock feed and waste, and the mulberry industry (Fig. 29) (Zhou et al. 2012). Although mushrooms can be cultivated using different methods, such as sawdust bags, bottles, shelves, and logs (see preceding chapters) there is an increasing demand for the establishment of modern mushroom facilities, which provides a range of $1 \sim 10$ million bags annually with well-equipped enclosed cooling and sterilized buildings. This enables proper growing conditions to be maintained. The most suitable conditions for mushroom production must be establish and are temperature, humidity, uniform ventilation, substrate moisture levels and or light to promote the formation of fruit bodies. For example, blue light-emitting diodes impact the quality of Lentinus sajor-caju (Huang et al. 2017a). However, maintaining and running such growing houses requires high volumes of electricity and water, making the production process costly and having a large impact on the release of greenhouse gases. Future trends should focus on the use of renewable energy sources.

The development of new technologies, such as photovoltaics for heating and cooling, artificial intelligent and technology for controlling the environment to optimize temperatures and moisture and even light formula by LED, will maximize the production season, enhance the productivity and quality of mushrooms, and reduce energy costs (Fig. 30). Artificial intelligence or automation includes substrate bag filling, inoculation, cultivation, scanning and picking up contaminated bags, and robots for packing and transportation. Both small- and large-scale production lines should embrace integrated systems, for example incorporating the use of recycled materials, such as agricultural waste, or making use of a sustainable supply 
of woody substrates from managed plantations, coupled with the integration of renewable energies.

Edible mushrooms have been cultivated for many centuries, and it is expected that their production will increase further due to market demand. The improvement and development of modern technologies, such as computerized control systems to control environmental parameters, automated harvesting, techniques for the production of mushrooms in a non-composted substrate, and new methods for substrate sterilization and spawn preparation, will increase the productivity of mushrooms (Sánchez 2004). However, the modern automated factory processes for mushroom production require a significant initial investment of fixed assets. Typically, this large capital outlay of fixed assets requires a recovery period of at least 5 years ( $\mathrm{Li}$ and $\mathrm{Hu}$ 2014). It is unreasonable for farmers in lowand middle-income countries to own automated equipment, since the upfront capital investment required for these items is economically unsustainable, even in the long term (Higgins et al. 2017). A more viable, long-term solution is the granting of funds to farmers via either public or privately funded organizations (Zied and Pardo-Giménez 2017).

\section{New edible mushrooms}

Due to their culinary, nutritional, and health benefits, the global market for mushrooms continues to grow, from US $\$ 34.1$ billion in 2015 to US $\$ 69.3$ billion by the end of 2024 (Valverde et al. 2015; Bal 2018). Mushrooms also show potential for use in waste management, as discussed elsewhere. However, mushrooms have life cycles very different from those of green plants. The choice of mushroom species for cultivation depends both on the growth media available and on market considerations (Beetz and Greer 1999; Rosmiza et al. 2016; Sánchez 2004). Oyster mushrooms, which grow on many substrates, are easiest (Patil et al. 1989). Shiitake mushrooms have already garnered considerable consumer demand (Teng 2008). To date, only two mycorrhizal mushrooms, morels (Morchella spp.) and truffles (Tuber spp.), have been commercially cultivated (Selosse et al. 2017).

Several new species of wild edible mushrooms have been successfully domesticated over the last few years, especially in tropical areas (Klomklung et al. 2012; Thongklang et al. 2014a, b, 2016; Rizal et al. 2016; Bandara et al. 2017) (Fig. 31A). Luangharn et al. (2017), Thongbai et al. (2017) and Klomklung et al. (2012) have shown that it is possible to domesticate local strains of Pleurotus giganteus that can grow at temperatures
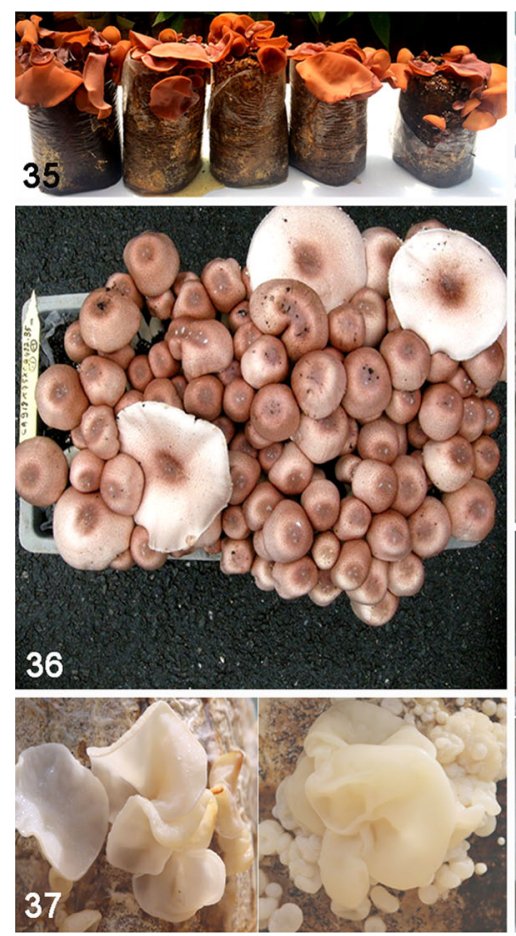
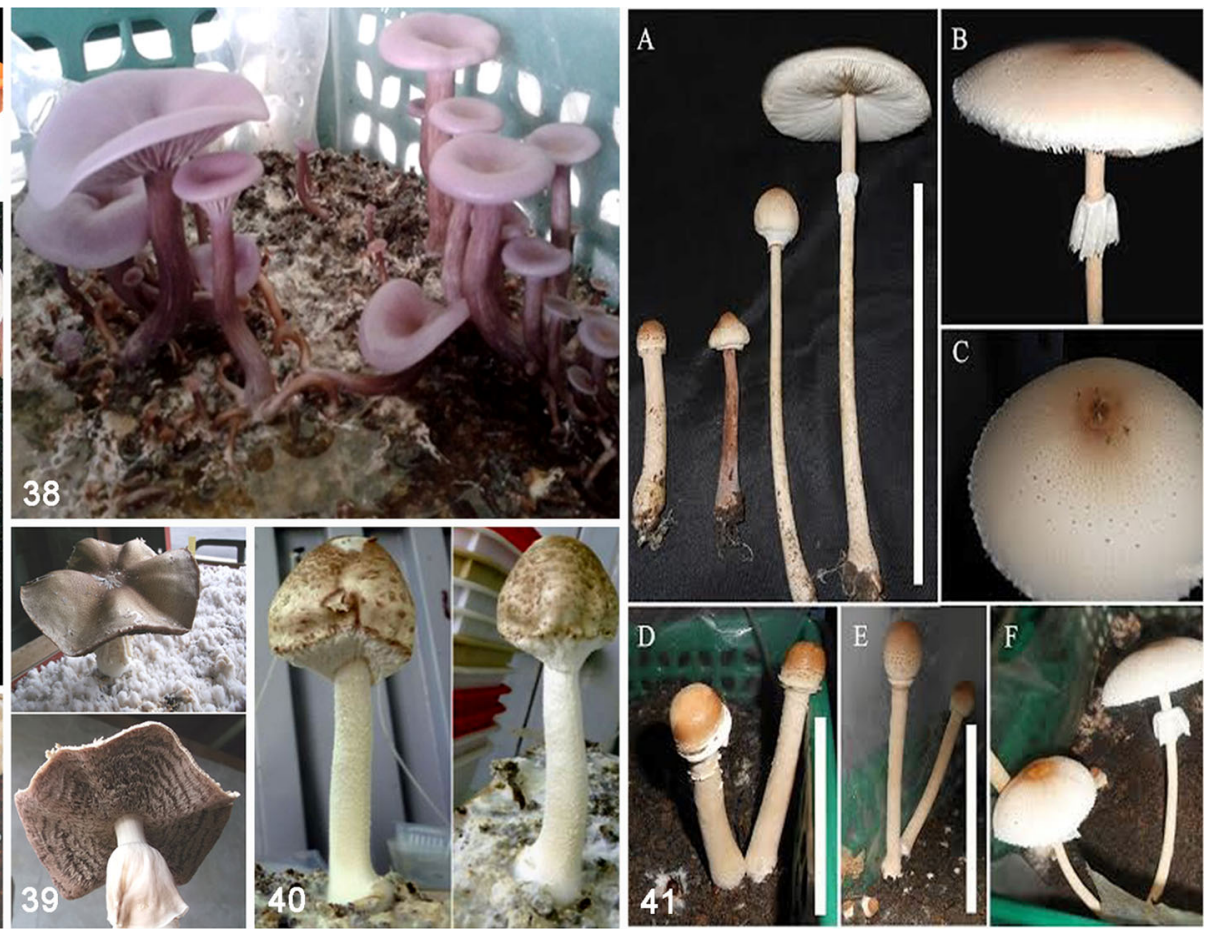

Fig. 31 A Auricularia thailandica; B Hybrid from Thai and French strains of Agaricus subrufescens; C White Auricularia cornea; D Lepista sordida; E Agaricus flocculosipes; F Agaricus subtilipes; G Fruiting bodies of Macrolepiota dolichaula strain MFLUCC-13-
0579. a different stages of fruiting body development, b pileus with annulus, $\mathbf{c}$ squamules on pileus, $\mathbf{d}$ and $\mathbf{e}$ bud stages of fruiting bodies; f mature fruiting bodies. $\mathrm{a}=25 \mathrm{~cm}, \mathrm{~b}=10 \mathrm{~cm}, \mathrm{c}, \mathrm{d}, \mathrm{e}, \mathrm{f}=5 \mathrm{~cm}$ 
consistent with Thai farm production. A new hybrid developed from Thai and French strains of Agaricus subrufescens was developed successfully between INRA, France and Mae Fah Luang University, which fructifies in tropical climates (Thongklang et al. 2014b, Fig. 31B). For the first time, a new white strain of Auricularia cornea was successfully domesticated at the Kunming Institute of Botany, Chinese Academy of Sciences (Fig. 31C). In Thailand, strains of Auricularia thailandica (Fig. 35), Lepista sordida (Fig. 31D), Agaricus flocculosipes (Fig. 31E), A. subtilipes (Fig. 31F) and Macrolepiota dolichaula (Fig. 31G) have successfully been cultivated. Mushrooms are not only used in traditional medicines, but are known to contain various bioactive components which can be used in cosmetics (Kwon and Thatithatgoon 2004; Hyde et al. 2010) and in medicine (De Silva 2013; Wisitrassameewong et al. 2012b). People in most parts of the world enjoy eating mushrooms, and therefore there is enormous potential for introducing new tropical mushrooms to the global market.

The number of fungi worldwide is estimated to be between 2.2 to 3.8 million, but only 120,000 species have been described, so it appears that about $92 \%$ of fungi have yet to be described (Hawksworth and Lücking 2017). Over the past 5 years, numerous new fungi have been described, especially within tropical areas (Ariyawansa et al. 2015; Liu et al. 2015; Li et al. 2016; Hyde et al. 2016; Tibpromma et al. 2017, 2018; Hyde et al. 2017; Wanasinghe et al. 2018). One recent paper published by Hyde et al. (2018a, b, c) showed that up to $96 \%$ of fungi in northern Thailand may be new to science. On the other hand, the State of the World's Fungi report 2018 (Willis 2018) states that about 350 species of edible fungi are collected and eaten worldwide annually, but this figure should be higher. To address this knowledge gap, the Center of Excellence in Fungal Research in Mae Fah Luang University and the Soil Biology group of the Kunming Institute of Botany are working on updating the global list of edible and medicinal fungi. There are many reasons for being optimistic about the future of wild edible fungi: they help maintain the health of forests and are a valuable source of nutrition and income.

\section{Agaricus subrufescens}

Agaricus subrufescens is an important medicinal mushroom (Wisitrassameewong et al. 2012a; De Silva et al. 2013), which belongs to section Arvenses (subgenus Flavoagaricus) of Agaricus. The main synonyms of $A$. subrufescens are A. blazei sensu Heinemann (misapplied), A. brasiliensis (illegitimate), and A. rufotegulis. This species has a broad climatic and geographical distribution range in Asia, Europe, Oceania and Africa (Thongklang et al. 2016). Agaricus subrufescens was discovered in
America in the late nineteenth century and later in Sao Paolo, Brazil; it was called Piedade mushroom. One mushroom (named A. blazei Murrill) was introduced in Asia in 1965 by T. Furomoto, who sent it to Japan for medicinal investigation but turned out to be conspecific with A. subrufescens (Kerrigan 2005). The common name for A. subrufescens is the almond mushroom. In addition, other common names are Himematsutake in Japan, Cogumelo do Sol in Brazil, and Royal Sun Agaricus in several other countries (Wisitrassameewong et al. 2012a, b). The main characters of $A$. subrufescens are a reddish-brown cap covered with silk-like fibres, a two-layered and floccose annulus, the odour of almond, yellow staining, and a positive Schäffer's reaction (Thongklang et al. 2014b).

Agaricus subrufescens has been commercially cultivated in Brazil, Japan, China, Korea and Taiwan (Kerrigan 2005; Gregori et al. 2008). In Brazil, the mushroom is a particularly important export mushroom with a higher price than other commercial mushrooms (Souza Dias et al. 2004). Button mushroom compost is normally used to cultivate A. subrufescens commercial strains. Other forms of agricultural waste are also used as alternative substrates to grow this mushroom. González Matute et al. (2011) produced A. subrufescens based on spent oyster mushroom substrate mixed with sunflower seed hulls, vermicompost and supplements. Wild strains of A. subrufescens have been studied for cultivation. Zied et al. (2011) reported the success of wheat straw with chicken manure and black peat + soil (4:1) as casing to cultivate a Brazilian/French A. subrufescens strain and their hybrids. Thongklang et al. (2014b) isolated a wild Thai A. subrufescens strain and successfully cultivated it in wheat straw/horse manure based compost at $25{ }^{\circ} \mathrm{C}$ and $95 \%$ humidity (Fig. 26a). Thongklang et al. (2014b) reported that samples from Brazil, France and Thailand are interfertile. Hybrid strains of Thai $\times$ Brazilian and Thai $\times$ French showed higher yields than Thai parental strains.

The mushroom is both nutritious and medicinal. Zied et al. (2017) evaluated the chemical composition of A. subrufescens, and reported that the stipe of the mushroom contained $69.56 \%$ of total carbohydrates, $63.89 \%$ of available carbohydrates, and an energy value of $363.97 \mathrm{kcal}^{100 \mathrm{~g}^{-1}}$ DM). The pilei comprised $33.96 \%$ protein, $7.75 \%$ nitrogen, 8.24 and $2.44 \%$ ash and crude fat. Medicinal benefits have also been reported for this mushroom, for example in cancer care, as antitumor agents, and in cholesterol reduction (Wisitrassameewong et al. 2012a, b; De Silva et al. 2013; Stadler and Hoffmeister 2015). Agaricus subrufescens produces several bioactive compounds, such as lectin, riboglucan, glucomannan, agaritine and blazien, all of which were shown to reduce tumor growth. In addition, spiro triterpenoids of the blazeispirol type are produced in mycelial cultures of this mushroom (Hirotani et al. 
1999, 2000, 2001, 2002). Extracts were found to be highly selective antagonists of Liver $X$ receptors (LXR) alpha (Grothe et al. 2011). Thongklang et al. (2017) reported that Thai-Brazilian and Thai-French hybrid strains produce higher yields of blazeispirol A in culture than their Thai parental strain. However, blazeispirols have never been found in the basidiomes of $A$. subrufescens (Thongklang et al. 2017). This is important, as if strains could be found with blazeispirols in the basidiomes, then eating the mushroom would have important medicinal benefits.

Mushrooms have been used as food and health products for millions years for their perceived medicinal properties. Agaricus subrufescens is widely cultivated in Brazil (Souza Dias et al. 2004; Mendonça et al. 2005), and the mushroom is exported from Brazil to various countries such as Australia, Bolivia, Germany, Korea, India, Japan, South Africa, Thailand, and the USA (Mendonça et al. 2005). The mushroom normally requires compost for cultivation (Thawthong et al. 2014). Agaricus subrufescens is consumed worldwide in fresh, dried and powder form from mycelium/fruiting bodies. Fresh mushrooms have been consumed as food because of their potential medicinal property and pleasant almond flavour. Moreover, dried or powdered mycelium/fruiting bodies are used as nutrient supplements. In Japan, 100,000$300,000 \mathrm{~kg}$ of dried almond mushrooms is produced every year (Takaku et al. 2001).

The mushrooms have been used as complementary and alternative medicine for cancer care. Private companies such as King Agaricus 100, Sen-Sei-Ro Gold, and ABMK sell products from $A$. subrufescens, and these products are currently being used by around 500,000 people for cancer treatment and prophylaxis (Hyodo et al. 2005).

\section{Using fungi to enhance food value}

For thousands of years, human societies have been utilizing fungi as food sources (Chang 1980; Moon and Lo 2014; Siddiq et al. 2018). In particular, edible mushrooms are ideal for vegetarians, because they are excellent sources of protein (typically 20-30\% crude proteins as a percentage of dry matter), have a low-fat content, are free of cholesterol, and contain most of the amino acids essential to human and animal nutrition (Kaur et al. 2018). Fungal taxa are utilized in the food industry taking advantage of their metabolism and metabolites (Gilbert and Robinson 1957; Moore and Chiu 2001; Adrio and Demain 2003; Ghorai et al. 2009). In particular, fungal species are used in the production of fermented foods and beverages in many traditional and indigenous cultures around the world (Abe et al. 2008; Dupont et al. 2016). For instance, the yeast Saccharomyces cerevisae is the oldest and best known fungal species used for wine and bread making, in drug production, as biocontrol agents, in enzyme biotechnology, as well as for research and development (Ghorai et al. 2009; Sharma et al. 2018). A brief description of some of the most important fermented food industries is provided below.

Mold-ripened cheese

Cheese is a solid or semi-solid protein food product manufactured from milk. Fungal species are important for the manufacture and ripening of two types of cheese, the Camembert and Blue-veined varieties (Fox et al. 2017). The name "blue-veined cheese" derives from the bluegreen mold growing throughout the product (Martín and Coton 2016). There are various kinds of blue-veined cheese products, viz. Roquefort, Gorgonzola, Stilton, Danish Blue, and Blue Cheshire. Most Blue cheese is produced from unpasteurized milk, and Penicillium roqueforti is added to the cheese prior to storage at controlled temperature and humidity. Penicillium roqueforti grows throughout the cheese and produces proteolytic and lipolytic enzymes (viz. proteases, lipase and beta-ketoacid decarboxylase) and methyl ketones, particularly 2-heptanone, as the major flavour and odour compounds (Kinsella et al. 1976; Dupont et al. 2016; Ropars et al. 2017). Penicillium roqueforti grows at a low oxygen tension and low $\mathrm{pH}$ values, while having the ability to use both pentoses and hexoses as substrate. These characteristics make the fungus ideally suited for the industrial Roquefort-type cheese production (Babel 1953; Kinsella et al. 1976, García-Estrada and Martín 2016). Camembert cheese is rather different from blue-veined cheese, as it characterized by a white mold growth on the surface and a soft texture. Penicillium candidum and $P$. camemberti are used for the camembert cheese production (Bourdichon et al. 2012).

\section{Fermented products}

\section{Soy sauce (Shoyu) and Miso}

Soy sauce is one of world's oldest flavor ingredients; made from fermented soybeans/wheat. It is a dark brown liquid that is stable at ambient temperature and does not require refrigeration during storage due to its low water activity and high salt content. Soy sauce has been used in cooking for over 1000 years, in particular in China, Japan, Korea and other Asian countries (Luh 1995; Hong et al. 2015; Liu 2017). The soy sauce production process comprises three major steps, which are Koji production, brine fermentation and refining. In the first step, soaked, cooked, mashed soy beans are mixed with an equal amount of roasted, lightly crushed wheat, and inoculated with $0.1-$ $0.2 \%$ starter mold (Aspergillus oryzae or A. sojae) in wooden trays. The fermented mixture (Koji) then undergoes brine fermentation using the lactic acid bacterium, Pediococcus halophiIus, and yeasts including Zygosaccharomyces rouxii and Candida species (Liu 2017). The final step of soy sauce fermentation (refining), includes pressing, filtration, pasteurization and packaging. The 
fermented soybean paste is called miso, has a similar aroma and flavor to soy sauce.

\section{Indonesian tempeh}

Tempeh is a mould-fermented product prepared from fermented soy beans and is consumed mostly in Indonesia (Babu et al. 2009). Tempeh possesses some unique characteristics, including its flavor, sliceable meat-like texture, and nutritional properties (Astuti et al. 2000; Nout and Kiers 2005). Rhizopus oligosporus is the dominant fungal species used in the soy bean fermentation process. Initially, husk-free, soaked and pressed soybeans are inoculated with $R$. oligosporus and fermented for 1-2 days. During the fermentation process, the white mycelia of $R$. oligosporus invade and cover the substrate mass to bind the soy bean (Shurtleff and Aoyagi 1979; Steinkraus 1995; Nout and Kiers 2005; Babu et al. 2009; Shah and Patel 2017). Tempeh provides many health benefits, in particular for heart diseases, strokes, osteoporosis, cancer and digestive disorders, loss of excess weight), as it contains essential fatty acids, numerous vitamins, fiber and minerals.

\section{Quorn}

Quorn is made from myco-proteins, which are produced by a fermentation process using the filamentous fungus Fusarium venenatum (Finnigan et al. 2017; Kozubal et al. 2019). Quorn mycoprotein strains were previously misidentified as Fusarium graminearum, but were later found to represent $F$. venenatum (Wheelock 1993; Trinci 1994; Wiebe 2004; Finnigan et al. 2017). With a low fat content and being free of cholesterol, quorn provides an alternative protein option that is meat-free and nutritious (Garodia et al. 2017). In addition, it is high in dietary fibre, which has been found to lower blood cholesterol (Denny et al. 2008; Ruxton and McMillan 2010; Garodia et al. 2017; Kozubal et al. 2019). The production process of Quorn is similar to that of beer, requiring less wheat and water for production, as well as resulting in lower carbon emissions, compared with meat sourced proteins (Finnigan 2011). During the production process, mycoprotein is mixed with vegetable flavorings and a small amount of egg albumen. To obtain its characteristic texture, a series of steaming, chilling and freezing processes are used to obtain the meat-like texture of Quorn products (Wiebe 2004; Finnigan 2011). Initially, Quorn products originated in the UK, but currently are available worldwide (Apostolidis and McLeay 2016).

\section{Rennet}

Rennet is used by food companies for the milk clotting process within cheese production (Ogel 2018). Calf rennet was traditionally used for cheese-making worldwide (Thakur et al. 1990; Mamo and Balasubramanian 2018). Subsequently, the global increase in cheese production, along with a decrease in animal rennet production, raised the price of traditional rennet. Therefore, food researchers tried to find alternatives for the milk clotting process within cheese production (Moschopoulou 2017; Ogel 2018). As a consequence, microbial rennet was produced using Rhizomucor miehei and R. pusilus (Thakur et al. 1990; Silveira et al. 2005; De Lima et al. 2008). Microbial rennet is cheaper than the rennet produced by animals, and the best option for vegetarians.

Other than those fermented food products, there are many commercial food industries in the world. Some food products which use fungal species are listed in Table 13 .

\section{Food colouring from filamentous fungi}

Synthetic colorants are widely used in food production to enhance the appearance of food colors. Although synthetic colorants are stable and inexpensive, the demand for these products has decreased due to their potential or perceived harmful effects on human health (Bateman et al. 2004). Today, many synthetic colorants have been replaced with safer natural colorants. Pigments from plants are often used as food colorants (Shamina et al. 2007; Ambati et al. 2014; Leong et al. 2018; Upadhyay 2018). Although these pigments can generate various colors, production processes are limited due to plant growth needs. Filamentous fungi can be grown in fermenters, and are also being investigated as important sources of pigments (Table 14, Fig. 32). Some examples are given below.

Red mold rice, also known as red yeast rice, is an Asian traditional fermentation product of steamed rice fermented with filamentous fungi belonging to the genus Monascus (Fig. 33). Red mold rice has been widely used in East Asian countries with a very long history as both food coloring and medicine. The red colour from red mold rice is used in a variety of Chinese, Korean and Japanese foods, such as fermented bean curd, preserved dry fish, pork stew, roast duck, and roast pork (Chen et al. 2015). Red mold rice is also used as substrate for the production of "Hong Qu glutinous rice wine," which gives it a bright-red colour and fine sweet flavor (Liu et al. 2018b; Park et al. 2016). After the optimization of biotechnological production processes, some species can easily produce pigments at a large scale in bioreactors, relying on relatively short fermentation times, and resulting in acceptable costs-ofgoods. For example, the optimization of red pigment production in a submerged culture by Monascus ruber in complex culture media resulted in the development of a medium containing $10 \mathrm{~g} / \mathrm{l}$ glucose, $5 \mathrm{~g} / \mathrm{l}$ corn steep liquor and $7.6 \mathrm{~g} / \mathrm{l}$ monosodium glutamate, generating the highest amount of red pigment at $20.7 \mathrm{U}$ (Hamano and Kilikian 2006). 
Table 13 Fungal species used in the food and beverage industries

\begin{tabular}{lll}
\hline Application & Product & Fungal species \\
\hline Beverages & Beer, Rum, Wine & Saccharomyces cerevisae \\
Cheeses & Sake & Aspergillus oryzae \\
& Roquefort, Blue cheese & Penicillium roqueforti \\
Oriental food fermentations & Camembert, Brie, soft ripened & Penicillium camemberti \\
& Ang-kak & Monascus purpureus \\
& Doenjang & Aspergillus oryzae \\
& Hamanatto & Aspergillus oryzae \\
& Miso & Aspergillus oryzae, A. sojae \\
& Ontjom & Neurospora intermedia \\
& Rennet & Rhizomucor miehei \\
& & Rhizomucor pusilus \\
& Shoyu (soy sauce) & Aspergillus oryzae, A. sojae \\
& Tempeh (Indonesian) & Rhizopus oligosporus \\
& Quorn & Fusarium venenatum \\
\hline
\end{tabular}

Table 14 Examples of pigments produced by filamentous fungi

\begin{tabular}{llll}
\hline Color & Pigments & Fungal producers & References \\
\hline Blue & Sanguinone A & Mycena sanguinolenta & Peters and Spiteller (2007) \\
Green & Xylindein & Chlorociboria aeruginosa & Saikawa et al. (2000) \\
Orange & Monascorubrin & Monascus sp. & Vendruscolo et al. (2017) \\
& Rubropunctatin & & \\
& $\beta$-Carotene & Mucor circinelloides Neurospora intermedia & Torres et al. (2016) \\
Red & Rubropunctamine & Monascus sp. & Hamano and Kilikian (2006), Dikshit and Tallapragada (2013) \\
& Monascorubramine & & \\
& Lycopene & Blakeslea trispora & Feofilova et al. (2006) \\
Yellow & Ankaflavin & Monascus sp. & Yongsmith et al. (2013), Klinsupa et al. (2016) \\
& Monascin & & \\
\hline
\end{tabular}

Red mold rice also generates orange pigment. Pigment production is partially associated with cell growth and a dissolved oxygen concentration of between 0.894 and $1.388 \mathrm{mg} \mathrm{O}_{2} / 1$ at $30{ }^{\circ} \mathrm{C}$. Limiting conditions of dissolved oxygen decreases the production of orange pigments (Vendruscolo et al. 2017). An induced mutation of $M$. purpureus led to production of yellow pigments, which can be added to Chinese fresh noodles, providing a yellow shade with no discoloration (Yongsmith et al. 2013). Klinsupa et al. (2016) investigated fusing intraspecific protoplasts of a yellow mutant of Monascus spp. with a white prototroph, and a strain with high yellow pigment production was produced. Another mutant strain of $M$. ruber 10910, generated through UV mutagenesis, improved the production of extracellular hydrophilic yellow pigment tenfold (Wu et al. 2015b).

Unfortunately, the mycotoxin citrinin is often found in red mold rice, and has a negative impact on health. This mycotoxin has been investigated as nephrotoxic and hepatotoxic to human cells (Bilgrami et al. 1988). The regulation of citrinin concentration in commercial red mold rice occurs in many countries, such as Commission Regulation (EU), Taiwan, US FAD, and Japan (Le Bloc'h et al. 2015; European Commission 2014). Therefore, the production of red mold rice with low citrinin is important to increasing its value and safety. Pengnoi et al. (2017) evaluated the effects of various purple rice varieties on the production of citrinin and red pigments by $M$. purpureus CMU002U (UV-mutant strain). The lowest value of citrinin concentration (132 ppb) was found in the Na variety, which passed the standards of Japan, Taiwan, and the European Union. The highest red pigment yield was obtained from the fermented Doi Muser variety. These results demonstrate that fermented purple rice has a high potential to be developed as a safe food colorant. 
Fig. 32 Chemical structures of pigments from filamentous fungi

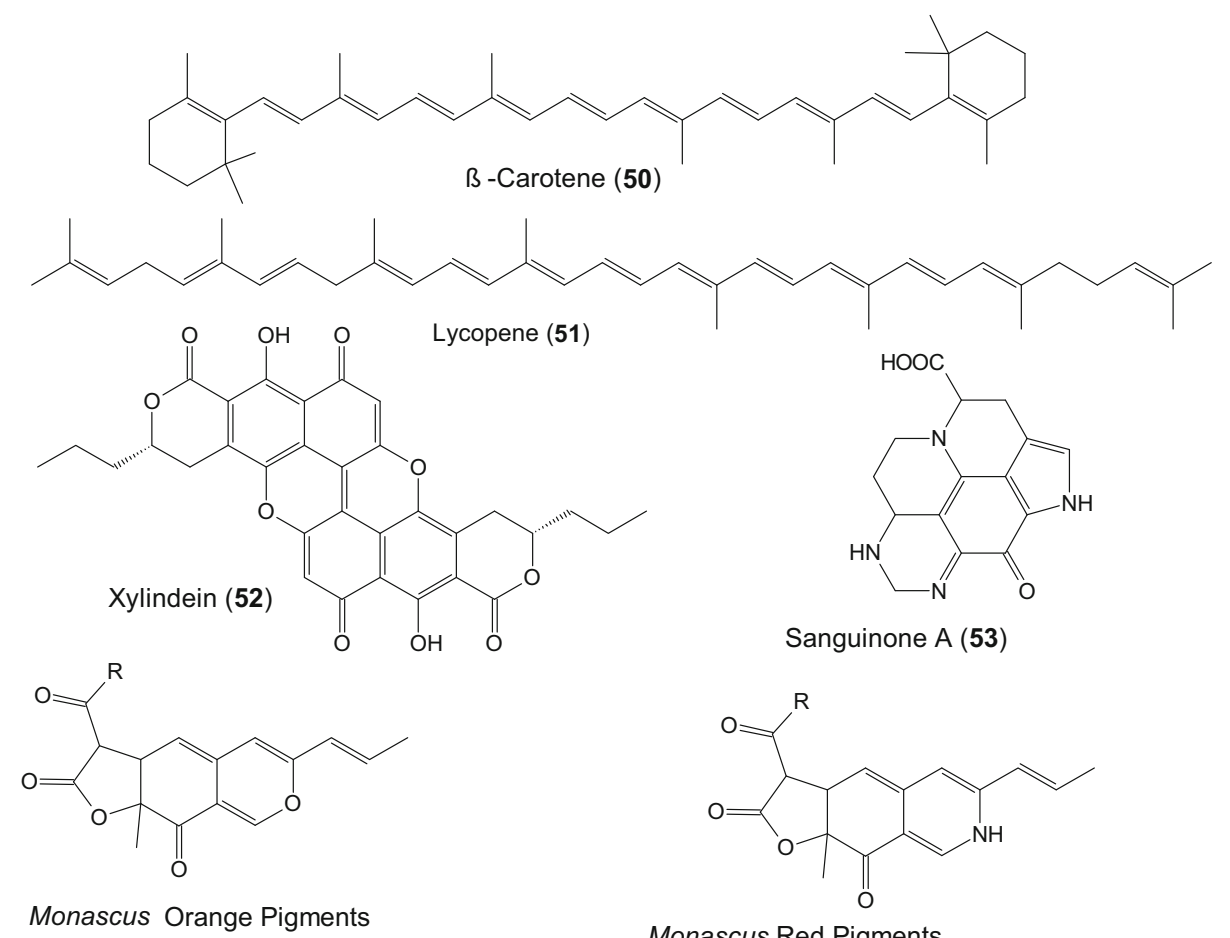

Monascus Orange Pigments

Monascorubrin, $\mathrm{R}=\mathrm{C}_{7} \mathrm{H}_{15}$ (54)

Rubropunctatin, $\mathrm{R}=\mathrm{C}_{5} \mathrm{H}_{11}$ (55)

Monascus Red Pigments

Rubropunctamine, $\mathrm{R}=\mathrm{C}_{5} \mathrm{H}_{11} \quad$ (56)

Monascorubramine, $\mathrm{R}=\mathrm{C}_{7} \mathrm{H}_{15}$ (57)

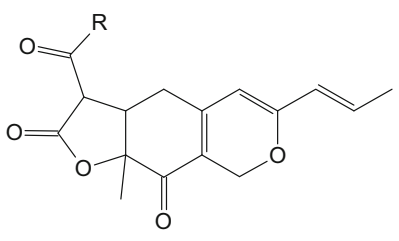

Monascus Yellow Pigments

Monascin, $\mathrm{R}=\mathrm{C}_{5} \mathrm{H}_{11} \quad$ (58)

Ankaflavin, $\mathrm{R}=\mathrm{C}_{7} \mathrm{H}_{15}$ (59)

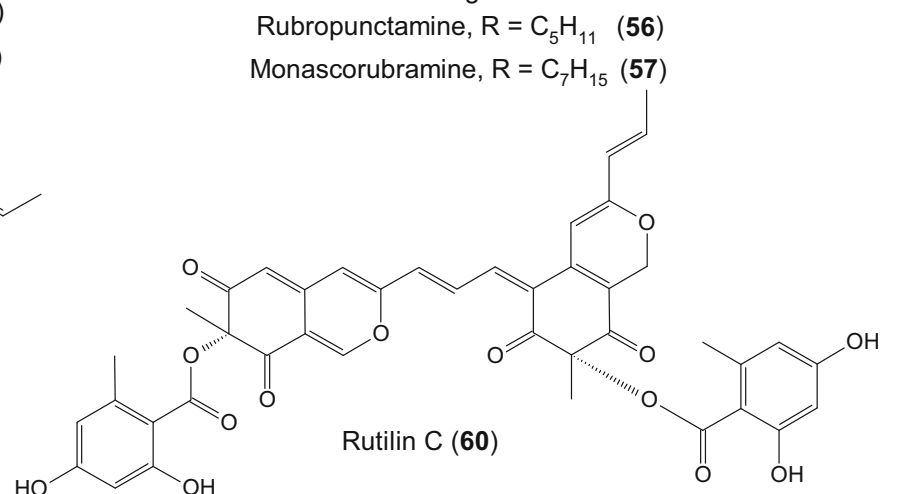

$\beta$-Carotene (50; orange pigment) is a precursor to vitamin $\mathrm{A}$, and of great importance for healthy skin, the immune system and vision, and is abundant in plants and fruits, such as carrots, pumpkin and sweet potatoes. This pigment is often added to various foods and drinks for its coloring effect and antioxidant activity (Hameed et al. 2017). The filamentous fungi Blakeslea trispora, Fusarium sporotrichioides, Mucor circinelloides, Neurospora crassa and Phycomyces blakesleeanus are all excellent producers of $\beta$-carotene (Torres et al. 2016). The pigment production by Neurospora intermedia was successfully manipulated by varying several factors. The formation of $\beta$-carotene was strongly influenced by light, carbon, $\mathrm{pH}$, and the cofactor zine ion. The highest pigmentation $(1.19 \pm 0.08 \mathrm{mg}$ carotenoids/g dry weight biomass) was achieved in a bubble column reactor (Gmose et al. 2018). Goksungur et al. (2002) investigated the effect of the pretreatment of molasses, nitrogen sources, natural oils, fatty acids, antioxidant, and precursors on $\beta$-carotene production by $B$. trispora. The maximum $\beta$-carotene concentration $\left(790.0 \mathrm{mg} / \mathrm{dm}^{3}\right)$ was obtained in culture grown in molasses solution supplemented with linoleic acid $\left(30.74 \mathrm{~g} / \mathrm{dm}^{3}\right)$, kerosene $\left(27.79 \mathrm{~g} / \mathrm{dm}^{3}\right)$ and antioxidant $\left(10.22 \mathrm{~g} / \mathrm{dm}^{3}\right)$. Thakur and Azmi (2013) investigated the different strategies for the extraction of $\beta$-carotene from Mucor azygosporus (strain MTCC 414). Various combinations of cell disruption and extraction methods were employed. The disruption of cells by sonication, followed by extraction with hexane and ethyl acetate $(1: 1, \mathrm{v} / \mathrm{v})$, gave a maximum yield of $\beta$-carotene $(985 \mu \mathrm{g} / \mathrm{ml})$. Zhang et al. (2016d) investigated a mutant strain of $M$. circinelloides for $\beta$ carotene production. The deletion in two mutants (MU206 and MU218) of the $\operatorname{crgA}$ gene, a well-known repressor of carotenoid biosynthesis, resulted in increased $\beta$-carotene 
Fig. 33 a Red mold rice produced by Monascus purpureus. b Monascus purpureus strain CMU002
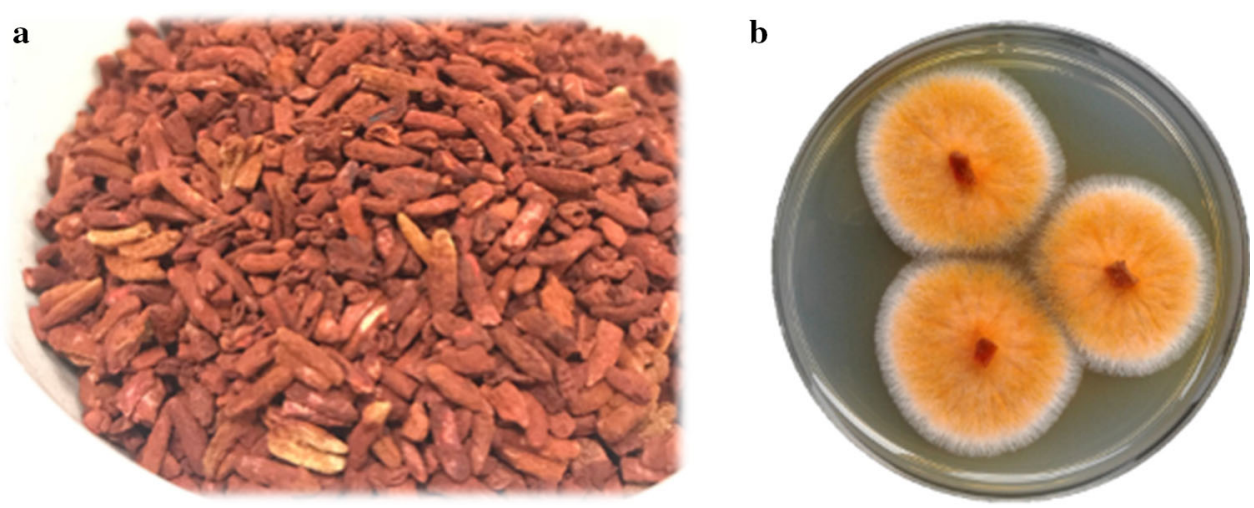

production. One strain derived from MU218 was able to accumulate up to $4 \mathrm{mg} / \mathrm{g}$ of $\beta$-carotene.

Lycopene (51; red pigment) is the strong natural antioxidant among carotenoids often found in tomatoes. Unlike $\beta$-carotene, it is not converted to vitamin A and remains in the blood plasma (Feofilova et al. 2006). The filamentous fungus Blakeslea trispora is a potential producer of lycopene. Enhanced lycopene production in $B$. trispora was achieved when treated with nitrogen ion implantation and N-methyl-N'-nitro-N-nitrosoguanidine. Mutant I5 with nitrogen ion implantation had a maximum lycopene yield of $28.8 \mathrm{mg} / \mathrm{g}$, which was $64 \%$ higher than the parent strain (Wang et al. 2013a). Shi et al. (2012) improved the lycopene production of $B$. trispora by adding isopentenyl alcohol, dimethyl allyl alcohol, geraniol, and mevalonic acid to the medium in optimal concentrations. The highest lycopene production in mated cultures was $578 \mathrm{mg} / \mathrm{l}$, achieved by adding $42 \mathrm{mg}$ geraniol/l to the medium after $48 \mathrm{~h}$ of growth. The optimization of lycopene production by $B$. trispora in bath culture was based on response surface methodology. The highest yield was observed at $50 \mathrm{mg} / \mathrm{l}$, by the addition of 2-methyl imidazole in a substrate supplemented with crude soybean oil (Pegklidou et al. 2008).

Many other species of filamentous fungi have the potential to produce various types of natural pigments for use in food coloring. However, to make these compounds commercially viable, much work on bioprocess technology and toxicity studies remains necessary before these pigments can be launched as products globally. Interestingly, the molecular background of biosynthesis of some fungal pigments, including the azaphilones from Monascus spp. (54-59; Mapari et al. 2010; Balakrishnan et al. 2013), has been elucidated, which may allow for a more straightforward and concise optimization of the production processes.

Aside from the above examples of fungal metabolites that are actually amenable to biotechnological production, there are many other pigments that have been described over the past century. A comprehensive overview of this topic is not possible, and therefore we refer to the reviews by Gill and Steglich (1987) and the updates by Gill (1994, 2003). Many fungal pigments have been shown to be heterogeneous mixtures of polymers, complexes of organic compounds with metal ions, or have been proven to be extremely unstable and can therefore not serve as a basis for the development of commercial colorants. The same holds true of stable compounds, which have so far only been encountered in ectomycorrhizal mushrooms that cannot be subjected to sustainable biotechnological production. In fact, some very interesting pigments have been discovered from saprotrophic fungi that have rather rare colors, such as the green pigment xylindein (52 in Fig. 32; Table 14, Saikawa et al. 2000) from Chlorociboria aeruginosa, or the blue pigment sanguinone A (53) from fruiting bodies of the mushroom Mycena sanguinolenta (Peters and Spiteller 2007). Since such green and blue pigments are especially in demand as natural food coloring agents, further research on these aspects may prove rewarding. Recently even over 1000 years old stromata of the ascomycete Hypoxylon fragiforme have yielded novel pigments such as rutilin C (60) (Surup et al. 2018), which can be isolated from freshly collected stromata in substantial amounts..

\section{Food flavouring}

Bio-flavors that are of natural origin are preferable and in higher demand when compared with chemically synthesized products, even though they often constitute the same compound, because of the phobias of consumers to synthetic food ingredients (Christen and López-Munguía 1994; Bluemke and Schrader 2001; Vandamme 2003; Ravasio et al. 2014). Plant extracts have previously been used as a main source of flavor and fragrance compounds (Carroll et al. 2016). Microbial fermentation can also be used to produce flavors and aromas (Kim 2005), such as aldehydes, esters, methyl ketones and terpenoids (Gupta et al. 2015; Carroll et al. 2016). 
Microbial cultures and their enzymes produce flavors naturally, as secondary metabolites of fermentation. Enzymes also act as biocatalysts in wide range of food flavor productions (Christen and López-Munguía 1994). These flavors are mostly used as food additives. The first synthetic flavor and fragrance compounds in the food industry were vanillin in 1874 and coumarin in 1868 (Gupta et al. 2015). Vanillin synthesis from ferulic acid uses a bioconversion process with two steps using two filamentous fungi (Lesage-Meessen et al. 1996). Ferulic acid is converted to vanillin acid using Aspergillus niger and reduced to vanillin using Pycnoporus cinnabarinus (Lesage-Meessen et al. 1996; Poornima and Preetha 2017).

Both filamentous fungi and yeasts play a significant role in producing flavoring compounds (Bluemke and Schrader 2001). In the wine industry, mixed fermentation of Lachancea thermotolerans and Saccharomyces cerevisiae are used to enhance the level of 2-phenylethanol, which has a rose-like odor (Ravasio et al. 2014). Saccharomyces cerevisiae and the filamentous basidiomycete Tyromyces chioneus are used for the production of grape wine and wine off flavors (Poornima and Preetha 2017). Off flavors are defined as "a flavor that is not natural or up to standard owing to deterioration or contamination" (Nijssen 1991; Christoph et al. 1999).

During amino acid catabolism in yeasts, isoamyl alcohol (banana flavor) and 2-phenylethanol (floral-rose flavor) are produced as natural flavors (Ravasio et al. 2014). Through the esterification of the lipase enzyme, banana, green apple and pineapple flavors are synthesized. Rhizomucor miehei is mainly used to obtain immobilized lipase (commercially available enzyme) in solvent free systems. This lipase was initially produced to be used in the food industry (Güvenç et al. 2002; Radzi et al. 2011; Goulet et al. 2012; Poornima and Preetha 2017). Through the lyophilisation of the culture fluid of Pleurotus sapidus (edible mushroom), the sesquiterpene (+)-valencene is deoxygenated into (+)nootkatone (citrus-type odor), which is an industrial flavor component (Krügener et al. 2010).

The main biotechnological techniques for food flavoring with microorganisms are de novo synthesis (fermentation) and biotransformation (bioconversion) (Krings and Berger 1998; Kim 2005). In de novo synthesis, simple, inexpensive substrates are used as starter media without adding precursors of the products. Many primary metabolites and mixtures of (small amounts of) many aroma compounds are normally produced in the media (Krings and Berger 1998). During biotransformation, precursors of the products are added to the cultivation media in large amounts, leading to production of a single major component (Kim 2005). Flavor active substances are considered to be volatile organic compounds, such as higher alcohols, esters, fatty acids derivatives and terpenes (Bluemke and Schrader 2001; Ravasio et al. 2014).

There are many commercialized enzymes for the biotechnological production of food flavor compounds, and a high concentration of aroma compounds. Enzymes are mainly used in food flavor synthesis because of the great reaction speed of catalytic activity in enzymes (Kim 2005). The contributions of fungi to flavor and aroma compound production are provided in Table 15.

Fungi produce a wide range of metabolites that can be used in the food industry (Vandamme 2003; Carrau et al. 2015). Microbial flavor phenotypes should be designed with the integration of natural yeast diversity, sensory analysis practices, and evolutionary engineering in mind (Carrau et al. 2015). Research should be implemented to better understand the molecular modifications of salivary composition, and receptor proteins and fats, which are stimulating and sensing odorants and tastes (Hofmann et al. 2018).

\section{What is mushroom stock? Products, processes and flavours}

Mushroom stock is a seasoning or food additive comprised of various mushroom flavours. In China, mushroom stocks are probably used more than anywhere else in the world. Mushroom stock products can be made from various species, including Agaricus bisporus (Guo et al. 2011), Termitomyces albuminosus (Zhou and Liu 2011) Tuber magnatum (Fantozzi et al. 2015) and many others. Mushroom stock products can mainly be divided into five types: condensed mushroom extracts, mushroom powders, mushroom sauces, preserved fried mushrooms, and instant mushroom soups. Mushroom extracts and powder are often used as a seasoning or ingredients in hot pot, while mushroom sauce and preserved fried mushrooms are commonly used as additives to rice, noodles and bread.

Several studies were carried out on processing technology, raw material ratio, nutrient composition and flavour components of mushroom stocks. Mushroom stock powder or liquids are made from various odorous mushrooms species. Normally, gas chromatography coupled with mass spectrometry (Largent et al. 1990; Rapior et al.2002; Du et al. 2014) and electronic nose technology (Fujioka et al. 2013; Zhou et al. 2015b) is usually used for the detection of volatile organic compounds (VOC) within mushrooms. Small hydrosoluble molecules, such as amino acids and nucleotides (Ribeiro et al. 2008; Wang et al. 2009), as well as volatile aroma substances, such as $\mathrm{C}_{8}$ derivatives, sulfurcontaining compounds, terpenes and other VOCs, are mainly associated with mushroom aroma (Rapior et al. 1996, 1997a, b, c, 2002; Liu et al. 2016; Palazzolo et al. 2017). These numerous potential combinations bring 
Table 15 Contribution of fungi or fungal enzymes to volatile organic compound production

\begin{tabular}{|c|c|c|}
\hline Fungi & Volatile organic compounds & References \\
\hline Aspergillus niger & Methyl ketones & Armstrong and Brown (1994) \\
\hline Aureobasidium pullulans & (creamy type odor) & Hagedorn and Kaphammer (1994) \\
\hline Penicillium sp. & & Krings and Berger (1998) \\
\hline Aspergillus niger & Vanillin & Ramachandra and Ravishankar (2000) \\
\hline Phanerochaete chrysosporium & (vanilla odor) & Poornima and Preetha (2017) \\
\hline \multicolumn{3}{|l|}{ Pycnoporus cinnabarinus } \\
\hline Beauveria bassiana & $\begin{array}{l}\text { Raspberry ketone } \\
\text { (berry type odor) }\end{array}$ & Gupta et al. (2015) \\
\hline Diutina rugosa (reported as "Candida") & $\begin{array}{l}\text { (-)-Menthol } \\
\text { (mint flavor) }\end{array}$ & Gupta et al. (2015) \\
\hline Ischnoderma benzoinum & $\begin{array}{l}\text { Benzaldehyde } \\
\text { (bitter almond flavor) } \\
\text { 4-Methoxybenzaldehyde } \\
\text { (coumarin-like odor) }\end{array}$ & $\begin{array}{l}\text { Fabre et al. (1996) } \\
\text { Gupta et al. (2015) } \\
\text { Krings and Berger (1998) }\end{array}$ \\
\hline Kluyveromyces sp. & $\begin{array}{l}\text { Phenylethanol } \\
\text { (rosary-like odor) }\end{array}$ & Gupta et al. (2015) \\
\hline Pithomyces sp. & Nor-patchoulenol (woody type odor) & Gupta et al. (2015) \\
\hline Yarrowia lipolytica & $\begin{array}{l}\gamma \text {-Decalactone } \\
\text { (peach flavor) }\end{array}$ & Gupta et al. (2015) \\
\hline Zygosaccharomyces rouxii & Furaneol (caramelly type odor) & $\begin{array}{l}\text { Hecquet et al. (1996) } \\
\text { Krings and Berger (1998) }\end{array}$ \\
\hline
\end{tabular}

intense and elegant flavour to the mushroom, e.g. an almond-like aroma from Agaricus subrufescens (Kerrigan 2005) and a maple syrup-like odour in Lactarius fragilis var. rubidus (Wood et al. 2012). These unique combinations can alleviate the potent smell of any meat, poultry, or seafood dish and are perfect choices for vegetarian and vegan dishes.

The rationale why mushroom stocks can be attractive ingredients foir many consumers is based not only on their flavour profile but also on a broad spectrum of their bioactive compounds (De Silva et al. 2013; Greeshma et al. 2018). These bioactive compounds may include unsaturated fatty acids (Günç et al. 2013), free amino acids (Ribeiro et al. 2008), volatile components (Politowicz et al. 2018), sulfur-sensory active compounds (Kupcová et al. 2018), antioxidant components like phenolics (Sevindik et al. 2018), antiproliferative and antitumor molecules like polysaccharides (Pandya et al. 2018; Morel et al. 2018), anti-lung cancer peptides (Prateep et al. 2017), and various neuroactive compounds (Thongbai et al. 2015; Sabaratnam and Phan 2017), depending on the mushrooms used to make the stock.

\section{Fungi used in making tea}

Tea is one of the most popular beverages in the world. Tea contains relatively high amounts of chemical substances, such as caffeine and vitamins, which can accelerate the oxidation of fat. Therefore, almost all teas have a slimming and weight loss effect. Fermentation is the key factor in the tea manufacturing process. The fermentation of tea leaves alters their chemistry, affecting the organoleptic qualities of the tea. Fermentation affects the smell of the tea and typically mellows its taste, reducing astringency and bitterness while improving mouthfeel and aftertaste. The microbes may also produce metabolites with health benefits. According to different degrees of fermentation, tea can be divided into green tea (non-fermented), oolong tea (semi-fermented), black tea (fully fermented by oxidizing enzymes) and dark tea (post-fermented by microbes). Unlike other teas, dark teas, such as Pu-erh tea and Ceylon tea, are microbially fermented teas.

Fungi play an important role in the post-fermentation process of dark teas. Fungi can change some sensory properties in the post-fermentative process, as well as influence the contents of polyphenols, catechins, gallic acid, and caffeine. Additionally, fungi can improve the ripping speed, success rate, and quality stability of the tea (Chen et al. 2008; Hou et al. 2010). Aspergillus and Blastobotrys spp. have been reported to be effective in enhancing the nutrition of $\mathrm{Pu}$-erh tea through fermentation (Zhao and Zhou 2005; Zhao et al. 2006; Abe et al. 2008). Some Aspergillus species, e.g. A. aureolatus, A. egyptiacus, A. japonicus, A. niger, A. penicillodes, A. restrictus, A. 
wentii var. fumeus and Penicillium spp., Rhizopus spp. and yeasts were isolated during Pu-erh tea processing (Chen et al. 1985, 2006). In addition to these fungal isolates, Zhang et al. (2016c) reported two new species, A. marvanovae and Candida mogii, from a solid-state fermentation of Pu-erh tea. Most fungal isolates can convert the tea polyphenols to bioactive theabrownins via a 4-day shaking fermentation in flasks at $40{ }^{\circ} \mathrm{C}, 250 \mathrm{rpm}$ (Zhang et al. 2016c).

Tea bushes grow only in tropical and subtropical climates. The time to pick tea leaves is related to the local temperature and humidity. For example, the picking period of Pu-erh tea is from the end of February to the end of November, and the best time for picking fresh leaves is half an hour after sunrise. Enzymatic inactivation is performed for freshly recovered leaves. After rolling and sun-drying, pile fermentation, the most important step, is carried out. Finally, the process of Pu-erh compressed tea (cake tea) is completed after a series of drying and compression steps. Water availability, temperature, and their interactions are important for mycelial growth. Chen et al. (1985) simulated the pile fermentation process of Pu-erh tea in the laboratory. During this process, Aspergillus niger, Penicillium spp. and Rhizopus spp. began to multiply at the beginning of fermentation. The maximum fungal quantity occurred in the first and second turning periods and then gradually decreased (Chen et al. 1985). Aspergillus niger is the predominant species in the whole pile fermentation, comprising about $80 \%$ of the mass of microorganisms (Chen et al. 1985; Liu et al. 1986); later, A. glaucus, a lowtemperature xerophilous species, begins to grow. Next, yeasts take a dominant position in the middle of pile fermentation (Zhao et al. 2015). The mass reproduction of molds and yeasts further suppresses the growth of bacteria (Liu 2009). The enzymatic activities of polyphenol oxidase, peroxidase and ascorbic acid oxidase are positively correlated with the growth status of fungi (Chen et al. 1985; Zhou et al. 2004; Zhao et al. 2015). High amounts of phenolic substrates, catechins and the oxidising enzymes can be released when the cellulolytic enzymes produced by fungi increase the maceration of plant tissues. Fungi are also involved in the transformation of components of $\mathrm{Pu}$ erh tea within the pile fermentation process. Wang et al. (2008) proposed that fungi can convert theophylline into caffeine. In addition, fungal metabolites are an important part of Pu-erh tea, for example in dimethoxy-4-methyl benzene, the specific metabolite of A. niger (Gong et al. 1993). However, toxic microbial metabolites were also detected from Pu-erh tea samples, such as Andrastin A, Emodin and Malformin C (Zhang et al. 2016c).

The commercially available tea also contains many fungal populations. Abe et al. (2008) found that Aspergillus niger and Blastobotrys adeninivorans exists in the final $\mathrm{Pu}-$ erh tea products. This is due to the influence of production environments and unstable fermentation processes, which are difficult to control and regulate. However, on the basis of traditional post-fermentation, through inoculating special preponderant fungal agents, not only can this suppress harmful microbes, but also can shorten the fermentation time and ensure the stability of product quality (Fig. 34).

\section{Wine, beer and spirits}

Brewing was invented about 8000 years ago, and became widely used as early as 5000 years ago (Corran 1975). At present, alcoholic beverages have become an integral part of numerous people's daily lives (Hornsey 2003, 2012). The three major well-known alcohol beverages are wine, beer and spirits, with beer being the most common and popular worldwide. These alcoholic beverages can be divided into many categories and subcategories based on their characters and fermentation processes. In the metabolic processes of brewing alcoholic beverages, the most important biological agents belong to the genus Saccharomyces. Some traditional beers have used the genera Candida, Dekkera, Saccharomycopsis and Schizosaccharomyces (Van Oevelen et al. 1977; Verachtert and Dawoud 1990) in production. Yeast has been used in the ethanol formation and the process to finish brewing wine, beer and spirits worldwide (Cambell 1996; Arora 2003; Kurtzman et al. 2011; Blomqvist 2011).

\section{Wine}

Wine seems to be the most easy to produce among the three beverages due to the fact that wine can be naturally fermented by sugar from ripe fruits and ambient yeasts. In the fermentation process, yeasts (natural or commercially cultured yeasts) use sugar from fruits, and release alcohol and carbon dioxide. Winemakers filtrate the large particles in the wine and bottle the wine or allow for additional aging (Fig. 35). However, the winemaking process can be complicated in cases of premium, healthy and specialty wine, e.g. types of grape fruits, oak-aging or steel tank, pumpovers or punch downs (Mosedale et al. 1999; Fischer et al. 2000; Zoecklein 2002a, b; Sacchi et al. 2005; Downey et al. 2006; Hicks 2011; Casassa and Sari 2014; Guerrini et al. 2017).

\section{Beer}

Beer is the most common alcoholic beverage, with the largest market share and consumption worldwide (Fig. 36). Goldammer (2008) reported that beer in the United States provides over $\$ 91.6$ billion U.S dollars annually in retail sales. The global market share of beer consumption has grown rapidly over the past decade worldwide. However, some reports state that people will tend to drink less beer in the near future due to a combination of factors, such as 
drinking-age population, GDP of countries and health concerns (IWSR, Economist.com 2017). Beer is mostly made from barley and other cereal grains, e.g. corn, rice, wheat. The brewing process is mainly about transforming carbohydrates from these grains into sugar. The primary product from grains is a sugary liquid called as "wort". Yeasts and wort will be fermented to produce alcohol and fizzy beers. This beverage was classified into two major categories, i.e. the "top-" (Saccharomyces cerevisiae) and the "bottom-" (S. pastorianus) fermenting types (Arora 2003). The top-fermenting type (or "ale") mostly produces darker beers, while the bottom-fermenting type (or "lager") produces lighter beers. Some other different characteristics

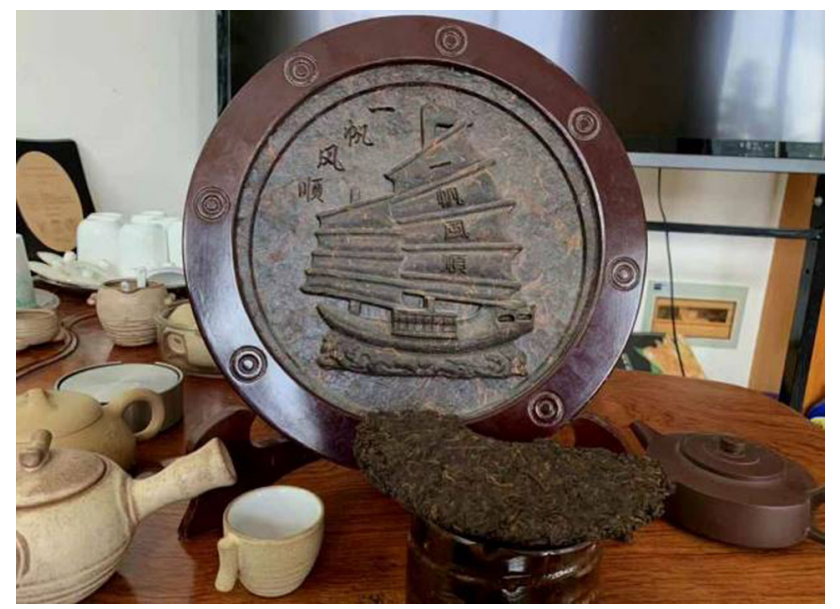

Fig. 34 Pu-erh compressed tea (cake tea) molded into a Table ornament. The compressed tea shown at the front is broken for use of ale and lager are shown in Table 16. Recently, hybrid and other specialty beers that have new flavors added or generated from the hybrid yeasts have been established and accommodated into the third category. For example, lager yeasts are cold-tolerant strains, but they have higher ethanol sensitivity than ale yeasts (warm-tolerant strains). Hybrid strains of regular $S$. cerevisiae $\times$ a cold-tolerant Saccharomyces species have been used to brew ale at lager (cool) temperatures (Magalhães et al. 2017). Some hybrids of yeast strains useful in the fermentation environment were produced, e.g. by Le Jeune et al. (2007), Gibson and Liti (2015), Krogerus et al. (2017). Presently, Saccharomyces contains hundreds of varieties and strains, which need to be studied further to understand the different expression of numerous genes for improving production in the brewing industry. In addition, many flavours have been recommended to add in the beer brewing process, including dried mushrooms. Many kinds of fungi have been tested and will be used in brewing in the near future (Okamura et al. 2001; Okamura-Matsui et al. 2003; Leskošek-Čukalović et al. 2010; Moon and Lo 2014).

\section{Spirits}

Hard alcohol or spirits have higher alcoholic content than beers and wines. Although yeasts can only survive at up to $15 \%$ alcohol by volume (Nguyen et al. 2009), a secondary process, "distilling," has been used to provide much stronger beverages than beers and wines. Brewers take advantage of the different boiling points of alcohol and other materials in the mixture to obtain spirits. Heating mixtures separates the alcohol as distilled liquids, while leaving water, grain particles and others in the boiling
Fig. 35 Pictures of the winemaking process by Jamie Goode (reproduced with kind permission by the publisher Jamie Goode, wineanorak.com)
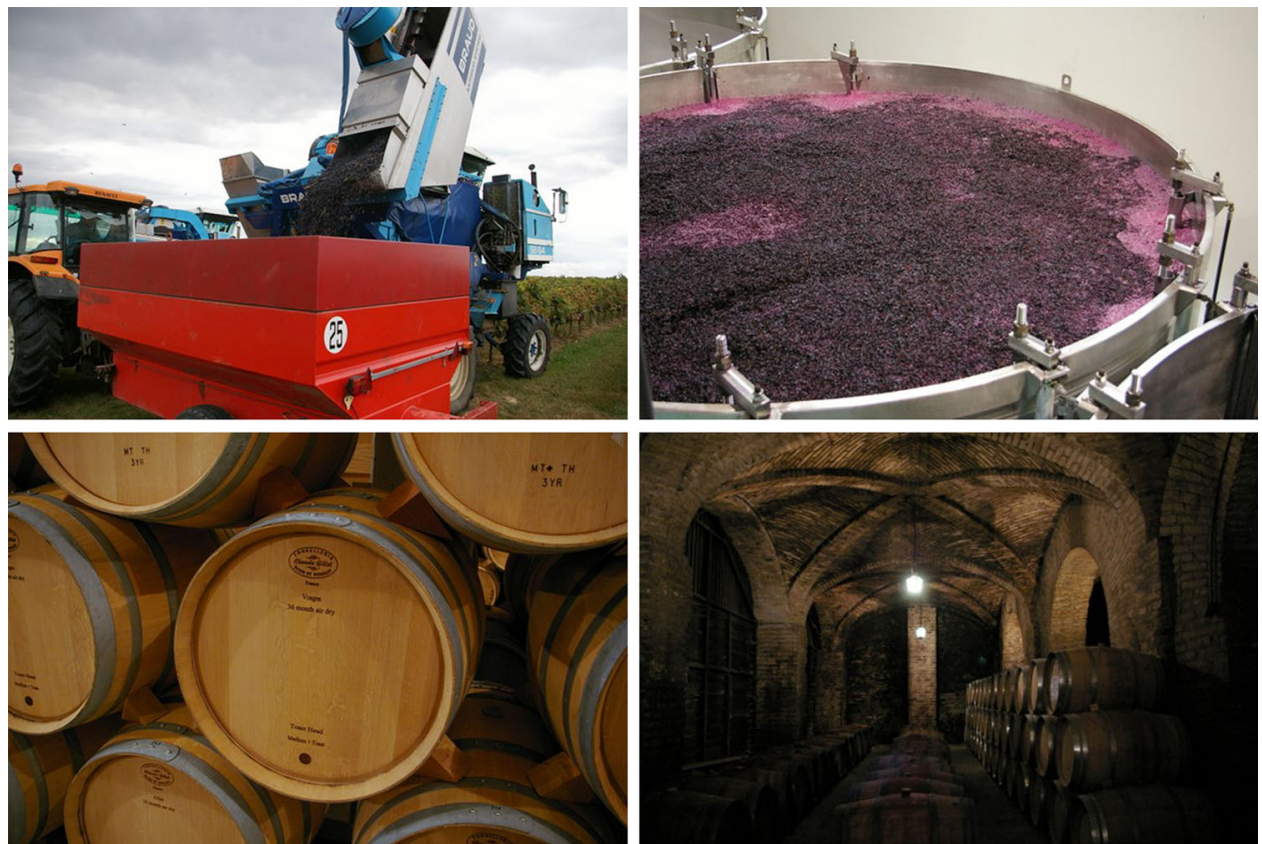


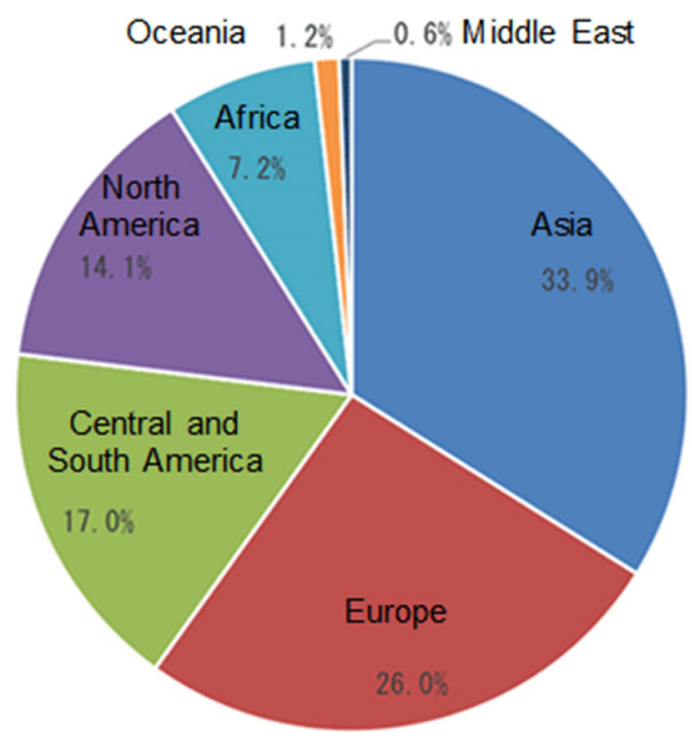

Fig. 36 Global market share of beer consumption in 2016 by regions (reprduced with kind permission from Kirin Holdings Company, Limited)

vessel. The liquids distilled are cooled in a condenser and often be re-boiled to ensure their purity (Forbes 1970; Lembeck 1983; Birch and Lindley 1985; Thomas and Shipman 2016). The raw materials result in a variety of flavours and aromas in the spirits, but the aging processes and periods are also important (Christoph and BauerChristoph 2007; Rosso et al. 2009; Robert 2010). The variety of spirits is showed in Table 17; however, there are many sub-categories within each type of spirit as well.

The brewed alcoholic beverage industry has grown rapidly over recent decades, and it is increasingly producing competitive products heightened by consumer requirements worldwide. Creating unique experiences for beer consumers is one of the most important goals of alcoholic beverage manufactories. Brewers have established numerous new techniques and processes for improving the flavours, colours, and nutrients in wines, beers, and spirits. Numerous new materials and processes in brewing that can affect flavours and aromas are being developed, and there will likely be hundreds of different kinds of alcoholic beverages in the future. The development of biotechnology and new discoveries will be the most important tools to reach the goals of future brewing.

\section{Functional foods and nutraceuticals}

The average age of the global population is increasing, and society is becoming more aware of the potential side effects of prescription medicines, and therefore are seeking alternative therapeutics (Ekor 2014; Rowe et al. 2016; WHO 2004a, b, 2015). Various new nutritional concepts, such as functional foods and nutraceuticals have been developed over recent decades. By definition, functional foods are conventional or everyday foods consumed as part of the normal daily diet, targeting the enhancement of the well-being and quality of life and thus reducing the risk of

Table 16 The major differences between ales and lagers

\begin{tabular}{|c|c|c|c|c|c|c|}
\hline $\begin{array}{l}\text { Beers } \\
\text { types }\end{array}$ & $\begin{array}{l}\text { Brewing } \\
\text { process }\end{array}$ & $\begin{array}{l}\text { Types of yeast } \\
\text { (Saccharomyces) }\end{array}$ & $\begin{array}{l}\text { Fermentation } \\
\text { temperature }\end{array}$ & $\begin{array}{l}\text { Brewing } \\
\text { times }\end{array}$ & Colour & Example \\
\hline Ale & Top-fermenting & S. cerevisiae & $\begin{array}{l}\text { Warm (15.5 to } \\
\left.25.5^{\circ} \mathrm{C}\right)\end{array}$ & 7 days & Brown to dark & $\begin{array}{l}\text { Goose Island } \\
\text { Bourbon County Stout } \\
\text { Samuel Smith's Taddy } \\
\text { Porter } \\
\text { Sierra Nevada } \\
\text { Three Floyds Dark Lord }\end{array}$ \\
\hline Lagers & $\begin{array}{l}\text { Bottom- } \\
\text { fermenting }\end{array}$ & S. pastorianus & $\mathrm{Cool}\left(7.2\right.$ to $\left.12.8^{\circ} \mathrm{C}\right)$ & $\begin{array}{l}\text { Several } \\
\text { months }\end{array}$ & $\begin{array}{l}\text { Blight gold or } \\
\text { yellow }\end{array}$ & $\begin{array}{l}\text { Carlsberg } \\
\text { Corona } \\
\text { Dunkel } \\
\text { Heineken } \\
\text { Kirin } \\
\text { Miller } \\
\text { Pilsner Urquell } \\
\text { Schwarzbier } \\
\text { Singha } \\
\text { Tsingtao }\end{array}$ \\
\hline
\end{tabular}


disease (El Sohaimy 2012). The term "nutraceuticals" is defined as food constituents that provide medical or health benefits, including the prevention and treatment of disease (Kalra 2003). Nutraceuticals can be food in the form of extracts, single, pure natural compounds, or nutrients that have been turned into a pharmaceutical formulation (e.g. pills or tablets), and they can be applied as dietary supplements or as part of a specific diet (Giavasis 2014; Reis et al. 2017). Therefore, the role of functional foods is mainly related to reducing the risk of disease, while nutraceuticals are usually consumed to promote well-being through the prevention or treatment of diseases and disorders (Bagchi et al. 2014).

Fungi, and especially edible mushrooms, are well known for their nutritional value and health-promoting properties. Mushrooms are ideal dietary supplements because they are relatively rich in proteins, contain almost no fat, and possess a variety of fibers and vitamins (Valverde et al. 2015). Many studies have been conducted to prove the benefits of functional foods and nutraceuticals derived from fungi in various pathological complications, such as diabetes, atherosclerosis, cardiovascular diseases, cancer, infection, renal, and gastrointestinal and neurological disorders (Banik et al. 2015).

Polysaccharides such as beta-glucanes are one of the most widely studied and recognized groups of bioactive molecules derived from mushrooms. For instance, the glucanes from Lentinula edodes (shiitake, Fig. 37) were successfully used as a partial replacement of wheat flour in baked foods to produce a low calorie, fiber-rich, functional food (Guillamón et al. 2010). Among all studied mushrooms species, L. edodes is also known to have antibacterial activities against both Gram-positive and Gram-negative bacteria (Alves et al. 2012a, b). In another study, the beta glucane lentinan (65, Fig. 38) of $L$. edodes was added to noodles as a partial wheat flour replacement, and it was claimed that their anti-oxidant and hypocholesterolemic effects in vitro would improve the quality of the noodles (Kalac 2013). Extracts from Ganoderma glucans, on the other hand, have been reported to act as free radical scavengers, thereby preventing lipid peroxidation, and to stimulate interferon synthesis in human blood cells after consumption (Kozarski et al. 2011).

In other studies, glucans from various edible mushrooms including Agaricus bisporus, Auricularia auricula-judae, and the Asian "Flammulina velutipes" (Enokitake: current name: F. filiformis; cf. Wang et al. 2018a), were shown to possess notable antioxidant capacities and free radical scavenging potential in vitro, and hence were proposed as natural antioxidants in food applications. Other snack foods enriched with a powder from extracts of the Chestnut Mushroom (Cyclocybe aegerita) as a partial starch replacement exhibited a low glycemic response after consumption, which was correlated to the dietary fiber content (Brennan et al. 2012). Agaricus subrufescens (synonyms: A. blazei; A. brasiliensis) is another mushroom which has been suggested as a safe immunostimulant and for ameliorating obesity or diabetes (Yamanaka et al. 2013). Lastly, Japanese researchers managed to produce a functional cheese-like food containing Schizophyllum commune. The final product contains a beta-glucan, which was claimed to have a significant antithrombotic function (Okamura-Matsui et al. 2001).

The term "mushroom nutraceuticals" was coined by Chang and Buswell (1996) to describe those compounds that have considerable potential as dietary supplements and are used for the enhancement of health and prevention of

Table 17 Differences of major types of spirits

\begin{tabular}{|c|c|c|c|c|c|c|}
\hline Spirits & Raw materials & Mashing & Fermentation & Distilling & Aging & Most \% ABV \\
\hline Brandy & Grapes and fruits etc. & $\checkmark$ & $\checkmark$ & $\begin{array}{l}\checkmark \text { (distilled } \\
\text { wine) }\end{array}$ & In oak & $40 \%$ (80 proof) \\
\hline Gin & Vodka + juniper berries & $\checkmark$ & $\checkmark$ & $\checkmark$ & $\times$ & $\begin{array}{l}40-47 \%(80-94 \\
\text { proof) }\end{array}$ \\
\hline Liqueur & $\begin{array}{l}\text { Spirits + sugar + flavor (fruit, cream, } \\
\text { herbs, spices, flowers or nuts) }\end{array}$ & $\checkmark$ & $\checkmark$ & $\checkmark$ & $\times / \sqrt{ }$ & $15-55 \%$ \\
\hline Rum & Sugarcane or Molasses & $\checkmark$ & $\checkmark$ & $\checkmark$ & $\begin{array}{l}\times \text { (light rum) } \\
\checkmark \text { in oak barrels (other rums) }\end{array}$ & $\begin{array}{l}40 \% \text { ( } 80 \text { proof), up to } \\
75 \% \text { ( } 150 \text { proof })\end{array}$ \\
\hline Tequila & Juices from blue Agave plant & $\checkmark$ & $\checkmark$ & $\begin{array}{l}\checkmark \text { (distilled } \\
\text { Agave) }\end{array}$ & $\begin{array}{l}\times \text { (white/silver tequila) } \\
\checkmark \text { in oak barrels (gold } \\
\text { tequila-blended) }\end{array}$ & $\begin{array}{l}40-50 \%(80-100 \\
\text { proof })\end{array}$ \\
\hline Vodka & Grains, rye, corn, wheat, potatoes etc. & $\checkmark$ & $\checkmark$ & $\checkmark$ & $\times$ & $\begin{array}{l}40-50 \%(80-100 \\
\text { proof) }\end{array}$ \\
\hline Whisky & Grains (corn, rye, wheat, barley, etc.) & $\checkmark$ & $\checkmark$ & $\begin{array}{l}\checkmark \text { (distilled } \\
\text { beer) }\end{array}$ & $\begin{array}{l}\checkmark \text { in charred oak, or used } \\
\text { whiskey or wine barrels }\end{array}$ & $\begin{array}{l}40-50 \%(80-100 \\
\text { proof) }\end{array}$ \\
\hline
\end{tabular}

Summarized from Graham (https://www.thespruceeats.com/quick-guide-to-distilled-spirits-760713) 
Fig. 37 Lentinula edodes (shiitake)
Fig. 38 Examples of

biologically active compounds from fungi used in functional foods and nutraceuticals. $\mathbf{6 1}$ : Synthetic compound inspired from cordycepin (Alapi and Fischer 2006); 62: Triterpenoid from Ganoderma sp. (Sato et al. 1986); 63: Main active principle of "Phellinus" (i.e.

Tropicoporus) linteus (Shao et al. 2015); 64: Blazeispirols (triterpenoid from cultures of Agaricus subrufescens

(Thongklang et al. 2017); 65: Lentinan, a beta-glucane from Lentinula edodes (Ina et al. 2013)

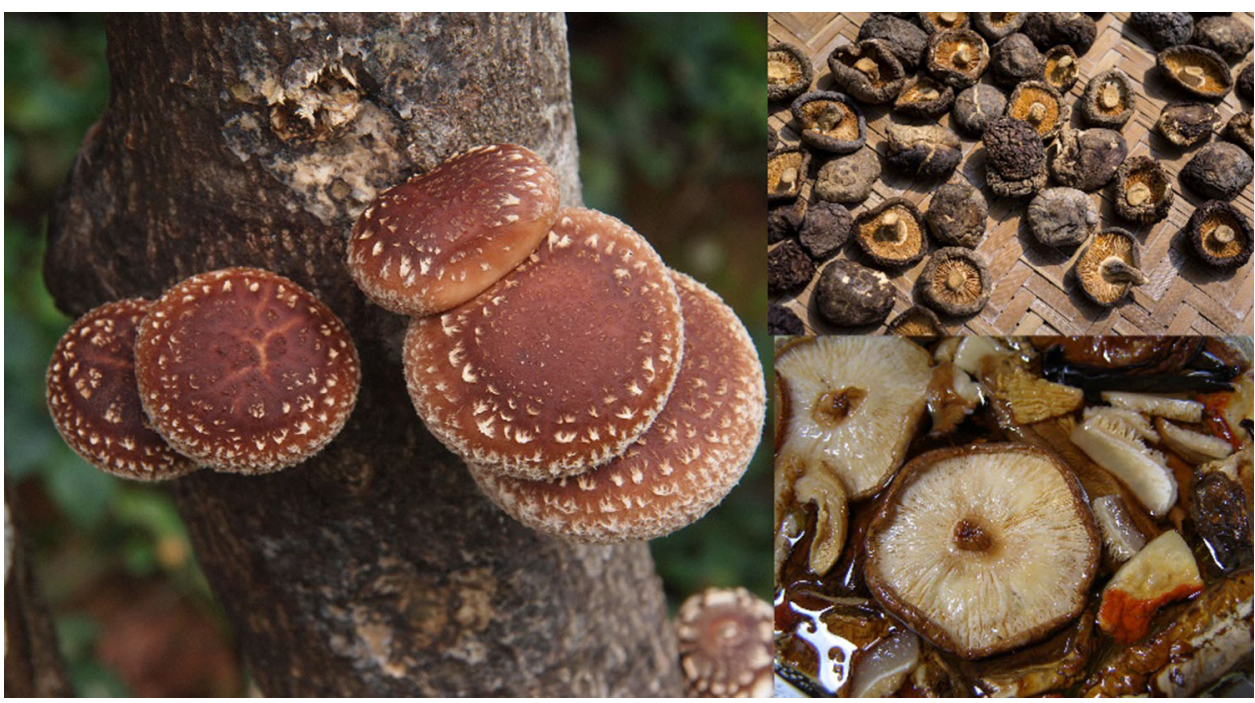<smiles></smiles>

Didanosine (61), Sythetic compound (based on the Coryceps spp compound) (Alapi EM and Fischer J 2006)<smiles>O=c1cc(O)cc(/C=C/c2ccc(O)c(O)c2)o1</smiles>

Hispidin (63), Phellinus linteus (Shao et al. 2015)<smiles>C[C@H](CC[C@H](O)C(C)(O)CO)C1CC[C@]2(C)C3=CCC4C(C)(C)[C@@H](O)CC[C@]4(C)C3=CC[C@]12C</smiles>

Ganoderiol A (62), Ganoderma sp. (Sato et al. 1986)<smiles>COc1cc2c(c(O)c1C/C=C(\C)CC(=O)CC(C)C)C(=O)OC2</smiles>

Hericenone A (64), Hericium erinaceus (Thongbai et al. 2015)

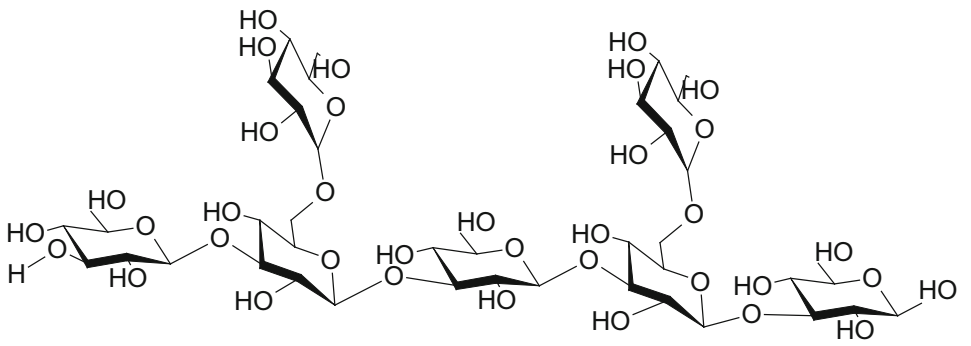

lentinan (65), Lentinula edodes

(Eaele et al.2015) 
various human diseases (Taofiq et al. 2016). The important edible mushrooms with remarkable nutraceuticals properties include the species of Auricularia, Grifola, Hericium, Lactarius, Pisolithus, Pleurotus and Tremella. Some studies suggest mushrooms as inhibitors of mycotoxin production (Reverberi et al. 2005). The bioactive glycoprotein (SX-fraction) extracted from Grifola frondosa, or maitake mushrooms, have been investigated in animal and clinical trials. It is particularly encouraging that the glycemic control of several diabetic patients under oral medications was significantly improved with a SX-fraction regimen that may primarily target or act on the insulin signal transduction pathway, overcoming insulin resistance (IR and IRS-1) (Konno et al. 2013). Other formulations exist on the market, such as a new product for dementia, particularly Alzheimer's disease, based on a proprietary standardized extract that contains hericenones (e.g. 64) and amyloban (both from $H$. erinaceus) (Thongbai et al. 2015). The popular culinary oyster mushroom Pleurotus ostreatus and other species of this genus synthesize bioactive pleuran, which is another potential candidate for the development of nutraceuticals (Giavasis and Biliaderis 2006; Zhang et al. 2001). Some examples of biologically active compounds from fungi used in functional foods and nutraceuticals are given in Fig. 38.

Today, it is very easy to find functional foods and nutraceuticals derived from mushrooms in the market. Many of these products can even be purchased via the Internet. However, it must be kept in mind that these studies do often not rely on standardized material and that the "health claims" derived from them are often not substantially justified, e.g. because the studies were conducted with rodents or even only in vitro (cell-based or enzyme based assays), and were never validated by treatment on humans in a similar manner as the clinical trials that are mandatory in development of ethical drugs. Serious diseases should therefore not be treated be self-medication and patients should by all means seek advice from a medical doctor. Among the various side effects reported for patients that have used such preparations from TCM mushrooms as self-medications, pancytopenia (i.e. a decrease in the number of all blood cells) has recently been frequently recorded (Yoon et al. 2011; Jung et al. 2013). Even though the symptoms disappeared after the consumption of the mushroom product stopped, these cases should give a warning to everybody who uses mushroom nutraceuticals not to overdose them.

\section{Harvesting the untapped probiotic potential of fungi}

Gastrointestinal disorders and diseases constitute a major cause of morbidity and mortality worldwide (Kaplan 2015;
Kirk et al. 2015; Peery et al. 2015). Diseases of the gastrointestinal tract may or may not be contagious. In the case of non-communicable gastrointestinal tract diseases, there is no transmissible organism involved. These include inflammatory bowel disease, irritable bowel syndrome, colon cancer, Crohn's disease and colitis. In the case of communicable gastrointestinal tract diseases, the aetiological agent is an infectious organism. Sufferers may exhibit one or several of a broad array of gastrointestinal tract disturbances and perturbations, including flatulence, abdominal pain, vomiting, nausea, gastrointestinal tract bleeding, constipation, and diarrhoea (Wadsworth et al. 2011).

Several gastrointestinal tract disorders are becoming increasingly difficult to treat due to ambiguous overall symptomatology or increased resistance of pathogens to administered pharmaceutical drugs, mainly antibiotics (Prestinaci et al. 2015). Given these issues, there is a critical need for therapeutics that will complement/replace existing strategies. In that light, probiotics present an attractive option. The Food and Agriculture Organization of the United Nations and the World Health Organization along with the International Scientific Association for Probiotics and Prebiotics define probiotics as "live microorganisms, which when administered in adequate amounts, confer a health benefit on the host" (FAO/WHO 2001; Hill et al. 2014).

Probiotics can be delivered as dietary supplements (e.g. capsules, tablets, and powder) or food ingredients (e.g. yogurts and kefirs) (Szajewska et al. 2016). The health benefits of probiotics include strengthening of the gut mucosal barrier followed by gut pathogen colonization resistance, replenishing of beneficial gut microbes after diarrhetic episodes, and enhancing overall health of the digestive tract (Hill et al. 2014). Probiotics have been used to a limited extent for the treatment/prevention of gastroenteritis, inflammatory bowel disease, irritable bowel syndrome, colorectal cancer, and colitis (Brown and Valiere 2004; Hill et al. 2014).

Most probiotics currently in use are limited to bacterial strains with members of the genera Bacillus, Bifidobacterium, Enterococcus, Lactobacillus, Propionibacterium and Streptococcus being the most common (Ouwehand et al. 2002; Brown and Valiere 2004; Szajewska et al. 2016). A notable exception is the yeast Saccharomyces boulardii, which was initially isolated to combat choleraassociated diarrhoea, and is now being used to treat diarrhoea of varying aetiology (McFarland and Bernasconi 1993). The health benefits of $S$. boulardii have been welldocumented in several randomized clinical trials (Table 18). Nonetheless, the probiotic potential of various other fungal species is also well known. For instance, members of the genera Kluyveromyces and Yarrowia are 
Table 18 Fungal probiotics and their health benefits

\begin{tabular}{lll}
\hline Fungi & Health benefits & References \\
\hline $\begin{array}{l}\text { Saccharomyces } \\
\text { boulardii }\end{array}$ & $\begin{array}{l}\text { Considerable reduction of duration of diarrhoea-RCT } \\
\end{array}$ & $\begin{array}{c}\text { Szajewska et al. (2016) and references } \\
\text { therein }\end{array}$ \\
& $\begin{array}{l}\text { Risk reduction of antibiotic-associated diarrhoea-RCT } \\
\text { children-RCT }\end{array}$ & \\
& Reduction of diarrhoea associated with Helicobacter pylori infection- & \\
& RCT & \\
& Amelioration of abdominal pain in IBS sufferers-RCT & Moslehi-Jenabian et al. (2010) \\
Saccharomyces & Absorption of mycotoxins & \\
\hline
\end{tabular}

$R C T$ randomized clinical trials

promising candidates due to their strong activity against bacterial pathogens and their ability to tolerate the inhospitable environment of the gut (Kumura et al. 2004; Chen et al. 2010). However, this avenue of research remains largely unexplored (Huseyin et al. 2017).

A largely untapped source of potential fungal probiotics is the human gut mycobiome, the collection of fungi residing in the gut. Members of the genera Candida and Saccharomyces are consistently prevalent across studies, but Cladosporium, Malassezia and Penicillium seem to be also abundant depending on the population under study (Hoffmann et al. 2013; Rodriguez et al. 2015; Nash et al. 2017). Future research should focus on identifying and precisely characterizing appropriate fungal probiotic strains. Outcomes of each probiotic fungal strain should be determined explicitly and optimal doses defined according to age group and disease state. Research efforts should also expand to examine the use of fungal probiotics in the aquaculture and animal husbandry industries.

\section{Saving the planet}

Waste is a common and major global problem. Anthropogenic activities result in the generation of millions of tons of plastic waste each year. Extensive agricultural practices have also contributed to the generation of enormous quantities of waste, which are being disposed of in non-cost-effective and environmentally unfriendly ways. There are various ways in which fungi can help decrease waste and reduce pollution. There is therefore an urgent need to tap into the power of fungal organisms and devise smart strategies regarding how they can reduce the negative effects that pollutants have on the environment. The section below outlines how fungi contribute to the health of our planet and have the ability to curb a number of environmental problems, most notably pollution. Examples are given as to how agricultural waste (which was previously disposed of or burnt, resulting in air pollution) can be converted into useful substrates on which to grow edible mushrooms at an industrial scale. Mycologists have also conducted research on resilient fungal microorganisms and explored their ability to degrade plastics, textile effluents, and hydrocarbons, all of which are polluting our environment at an alarming rate.

\section{Agricultural waste disposal}

Agricultural waste, the residue from planting until harvest, generally contains a mixture of plant waste, animal/insect waste, and toxic residues from the chemicals used as pesticides, insecticides, and herbicides. Asia is one of the world's most agriculture-intensive areas: from 2010 to 2016, 46.9\% of emissions in Asia came from crop residue, compared to $28.2 \%$ and $16.2 \%$ from America and Europe, respectively. According to the FAOSTAT $^{2}$ emissions database, $90.6 \%$ of rice produced within Asia generated more than a hundred million tons of residual material. This agricultural waste accounted for over $50 \%$ of the nitrous oxide generated from the decomposition of crop residue on cultivated soils, one of the main contributors of the greenhouse gas effect and a cause of global warming. In the Sacramento Valley of California, rice and wheat straw are also major contributors of generated agricultural waste, with 1.5 tons of rice straw waste produced annually (Zhang et al. 2002). In Thailand, the main crops are sugarcane, cereal, rice, cassava and oil palm, and these contribute $80 \%$ of the total solid waste generated from crop cultivation.

A number of processes have been developed or proposed for agricultural waste management (such as composting, which is a sustainable process), while the common practice of burning the rice straw, husk, stalks, corn cobs, stubble and grasses is considered a cheap and easy means for the disposal of excess residues (Obi et al. 2016; Walia et al. 1999). Unfortunately, burning agricultural waste generates many poisonous and harmful oxides and hydrocarbonates into the atmosphere (Shaban and Omaima

\footnotetext{
${ }^{2}$ http://www.fao.org/faostat/en/?\#data/GA.
} 
2010), which in turn increases air pollution and causes respiratory problems, which then impact the economy through higher medical costs and employee absences. Therefore, there is an increasing demand for sustainable processes that utilize agricultural waste in more ecofriendly ways.

Biological processes are considered both natural and sustainable, and are also important in carbon recycling. Fungi are some of the most effective decomposers in an ecosystem and are extremely efficient in the degradation of plant-based agricultural waste such as rice straw, wheat straw, maize stoves and sugarcane residue (Dinis et al. 2009). Mushroom cultivation using agricultural waste, e.g. wheat, rice, paddy straw, rice bran, coffee straw, tea leaves, cotton straw sawdust, provide profitable agri-business opportunities for small farmers and offers an eco-friendly alternative to disposing of waste, which is normally burnt (Zhang et al. 2002). Various commercial mushrooms are cultivated worldwide, and agricultural residues are used as cheap and renewable substrates, and a selection is given in Table 19.

Agricultural waste consists of large quantities of lignocellulosic waste, and is composed of approximately 60 $70 \%$ fermentable sugars and $20-30 \%$ aromatic compounds. Lignocellulosic waste is a renewable and important source for biomass/ethanol production (Kim and Dale 2004). Wheat straw, corn stover, sugarcane bagasse, and rice straw are the most common substrates for biofuel production, and filamentous fungi are key components of the fermentation process. Pichia stipites (strain NRRL
Y-7124) is used in the fermentation process to produce ethanol from wheat straw and rice straw (Moniruzzaman 1995), and Fusarium oxysporum, Neurospora crassa, and Paecilomyces sp. are typically used to ferment cellulose directly to ethanol (Szczodrak and Fiedurek 1996). Therefore, various fungi have the potential to produce lignocellulosic enzymes and play important roles in ecoagricultural waste practice. White-rot fungi can also be used to pre-treat the agricultural waste to enhance the enzymatic saccharification process due to the selective removal of lignin (Miyauchi et al. 2018; Rouches et al. 2018; Zhou et al. 2015b, 2018). The screening of the secreted enzymes from fungi can also be used to enhance the saccharification of agricultural waste by commercial enzyme cocktails, and this approach has led to the discovery of new activities such as lytic polysaccharide oxidases which open up the lignocellulose structures to facilitate further access of cellulolytic enzymes (Berrin et al. 2012; Couturier et al. (2012, 2018). Alternatively, fungi can be used to transform compounds in agricultural waste into higher value commercial molecules such as vanillin (Taira et al. 2018) and cannolo (Odinot et al. 2017).

In most countries, some waste, such as cereal residue, is universal, whereas other forms of agricultural waste are more region-specific. In the French region of Provence, for example, lavender is grown on a large scale for the production of essential oils. Globally, these oils are among those most commonly used for industrial purposes, including in perfumes, pharmaceuticals and cosmetics.

Table 19 Agricultural waste use as substrate for mushroom cultivation

\begin{tabular}{|c|c|c|c|}
\hline Fungal taxa & & Agricultural waste & References \\
\hline $\begin{array}{l}\text { Agaricus } \\
\text { bisporus }\end{array}$ & $\begin{array}{l}\text { Button } \\
\text { mushroom }\end{array}$ & Wheat straw & $\begin{array}{l}\text { Kamthan and Tiwari } \\
\text { (2017) }\end{array}$ \\
\hline $\begin{array}{l}\text { Ganoderma } \\
\text { sp. }\end{array}$ & $\begin{array}{l}\text { Chinese } \\
\text { mushroom }\end{array}$ & Sawdust & Shashitha et al. (2016) \\
\hline $\begin{array}{l}\text { Lentinula } \\
\text { edodes }\end{array}$ & $\begin{array}{l}\text { Shiitake } \\
\text { mushroom }\end{array}$ & $\begin{array}{l}\text { Bracts of pineapple crown, sugarcane bagasse, sugarcane leaves, wheat straw, corn- } \\
\text { cobs, oak-wood sawdust }\end{array}$ & $\begin{array}{l}\text { Salmones et al. (1999) } \\
\text { Philippoussis et al. } \\
\text { (2007) }\end{array}$ \\
\hline $\begin{array}{l}\text { Pleurotus } \\
\text { eryngii }\end{array}$ & $\begin{array}{l}\text { King oyster } \\
\text { mushroom }\end{array}$ & $\begin{array}{l}\text { Supplementation of wheat bran with wood chips. Barley straw and sugar beet pulp with } \\
\text { rice bran }\end{array}$ & Jeznabadi et al. (2016) \\
\hline $\begin{array}{l}\text { Pleurotus } \\
\text { djamor }\end{array}$ & $\begin{array}{l}\text { Pink oyster } \\
\text { mushroom }\end{array}$ & Supplementation of wheat bran with sugarcane bagasse & Hasan et al. (2015) \\
\hline $\begin{array}{l}\text { Pleurotus } \\
\text { ostreatus }\end{array}$ & $\begin{array}{l}\text { Oyster } \\
\text { mushroom }\end{array}$ & $\begin{array}{l}\text { Paddy straw, rice straw wheat straw, date-palm leaves empty fruit bunch, olive cake, } \\
\text { tomato tuff, banana leaves, pine needles, sugarcane bagasse }\end{array}$ & $\begin{array}{l}\text { Alananbeh et al. } \\
\text { (2014) } \\
\text { Ananbeh and } \\
\text { Almomany } \\
(2005,2008) \\
\text { Ali et al. (2010) } \\
\text { Marlina et al. (2015) } \\
\text { Rezania et al. (2017) } \\
\text { Yang et al. (2013a) }\end{array}$ \\
\hline
\end{tabular}


After steam distillation, the residual straw is considered to be agricultural waste, though it can also be used as a source of terpene derivatives (e.g. $\tau$-cadinol, $\beta$-caryophyllene), lactones (e.g. coumarin, herniarin), and phenolic compounds of industrial interest, including rosmarinic acid (through fungal treatments) (Lesage-Meessen et al. 2018). Fungal-derived enzymes represent over $60 \%$ of the world market in industrial enzymes. Lavender residues, like most agricultural waste residues, are both suitable catalysts for the secretion of a wide panel of lignocellulose-acting enzymes, such as cellulases, laccases and xylanases, and therefore can provide a local and cheap source of degradative and transforming biocatalysts. Even plant pathogens like Phyllosticta capitalensis produce arabinase, cellulase, laccase, pectinase and xylanase, especially when cultivated on lavender residues. The initial growth of $P$. capitalensis on lavender waste was higher in comparison to its growth on other agricultural waste materials, such as miscanthus, rice straw, rice husk, sorghum, and wheat straw, suggesting further stimulation of growth by a compound remaining within the lavender residue (Wikee et al. 2017). This opens up further avenues to investigate the presence of microbial growth enhancers within agricultural waste for utilization in biotechnology. Therefore, the aims of this present review are to reveal the capacity of fungi for agricultural waste management and to explore the varieties of fungi and their lignocellulolytic production, taking full advantage of various agricultural waste products as substrate.

\section{Mycoremediation: Fungi to the rescue}

Man-made activities have resulted in extreme environmental pollution, and therefore environmentally friendly remediation techniques are more necessary than ever before (Rhodes 2013). Fungi, the most vigorous decomposers found in nature, are a potential solution to this problem (Singh 2006). Bioremediation with fungi, known as mycoremediation, is an innovative biotechnological approach which uses live fungi to clean up contamination through total mineralization using enzymes, or through the total removal of the contaminant via absorption (Stamets 2005). Pollutants released into the environment due to manmade activities pose a significant threat to the sustainability of the environment and to human health. The burning of wood and fossil fuels, as well as coal mining and oil drilling all release recalcitrant pollutants such as polycyclic aromatic hydrocarbons (PAH), which are not easy to remove once they have been released. In addition, pesticides that leak into natural waterways and industrial effluents containing heavy metals, that have been released into the environment without proper treatment can be effectively removed if fungi are utilized as a remediation tool.
The vegetative part of the fungi, called mycelium, has the ability to exude powerful extracellular enzymes and acids that are able to decompose many organic substrates, including lignin and cellulose (Pointing et al. 2001; Bucher et al. 2004). With a simple screening procedure, the most suitable fungal species for the target pollutant can be identified (Matsubara et al. 2006). Many species of fungi, such as Aspergillus niger, A. terreus, Cladosporium oxysporum, Fusarium ventricosum, Rhizopus oryzae, Trichoderma harzianum as well as the model white rot fungus, Phanerochaete chrysosporium, have been tested for their ability to degrade pesticides (Bhalerao and Puranik 2007; León-Santiesteban et al. 2016). Polychlorinated biphenyls, which are highly toxic chemicals previously used in many industries and one of the more persistent organopollutants, can be degraded by non-ligninolytic enzymes from fungi such as Fusarium solani, Penicillium chrysogenum and Scedosporium apiospermum (Tigini et al. 2009).

Different species of basidomycete white rot fungi are best suited for mycoremediation, as their extracellular lignin modifying enzymes have low substrate specificity (Lang et al. 1995). Not only do they target lignin, but they also are capable of targeting many organic pollutants that are similar in structure to lignin, like DDT, lindane, chlorodane and PCBs (Arisoy 1998; Pointing 2001; Mansur et al. 2017). Enzymes like lignin peroxidases (LiP), manganese peroxidases (MnP) and laccases (LAC) secreted by white rot fungi are excellent at degrading organopollutants held together by hydrocarbon bonds (Kües 2015). White rot fungi such as Lentinus subnudus, Phlebia acanthocystis and Pleurotus ostreatus, and many others have been successfully used separately as well as in mixtures to treat sites contaminated with pesticides and herbicides (Kamei et al. 2011; Nyakundi et al. 2011; Xiao et al. 2011). Since white rot fungi grow by hyphal extension, they can better reach the pollutants in contaminated sites, unlike other organisms with a low colonizing capacity (Reddy and Mathew 2001). Furthermore, the lignin degrading enzymes used by white rot fungi are extracellular (Novotný et al. 2004). These fungi can tolerate high concentrations of the pollutant, as the pollutant does not need to be internalized in order to start the degradation process.

Lamar and White (2001) recommend a four-step strategy for the practical implementation of fungi for the mycoremediation of contaminated sites. The steps include laboratory scale experiments to establish preparation methods; on-site pilot testing to understand sound technical and engineering techniques; and the production of inoculum fortified with nutrients to ensure growth and finally full-scale application (Fig. 39).

Because of their tolerance to extreme $\mathrm{pH}$ conditions, temperatures, and nutrient availability, fungal species such 
as Aspergillus sp. and Sterigmatomyces halophilus have been tested for their ability to absorb heavy metals from contaminated sites (Bano et al. 2018). The cell walls of these fungi have excellent metal binding capabilities, and can absorb contaminants which then can be physically removed by harvesting the fungus (Baldrian 2003). The utilization of the biosurfactants of fungal species has been a success for the removal of heavy metals such as Fe, Zn and $\mathrm{Pb}$ (Igiri et al. 2018). For example, an anionic biosurfactant from Candida sphaerica was tested on cleaning soil collected from an automotive battery industry, and the heavy metal removal success rate was $95 \%, 90 \%$ and $79 \%$ for $\mathrm{Fe}$, $\mathrm{Zn}$ and $\mathrm{Pb}$, respectively (Luna et al. 2016).

The textile industry is one of the primary contributors to environmental pollution. Untreated textile effluents, inefficiency in the dyeing process, and the poor handling of spent dye stuff effluents are the main causes of surrounding natural water and land contamination, both of which adversely affect plant, human and animal life. Textile dyes, such as azo dyes (acid red) or anthraquinonic dyes (basic blue) are highly toxic and mutagenic in nature (Delclos et al. 1984). Biological treatment of these harmful pollutants through microbial decolourization and degradation have attracted attention as the conventional treatment methods produce hazardous by-products and are not very cost effective. Trichoderma harzianum (Singh and Singh 2011) has been successfully bench tested for the decontamination of various textile dyes.

Apart from the absorbance process, enzymatic degradation of waste is another feasible approach that can be used for the removal of slow-degrading waste. Various types of cellulose-based urban solid waste are primarily disposed of via incineration. One study conducted by Espinosa-Valdemar et al. (2011) used Pleurotus ostreatus (Oyster mushroom) to reduce the mass and volume of used disposable diapers via cellulose degrading enzymes secreted by the fungi, which simultaneously produced a pathogen-free, high-protein food source in the form of a mushroom fruiting body that could be used for human consumption or as a food supplement for animals.
Fig. 39 The process of mycoremediation
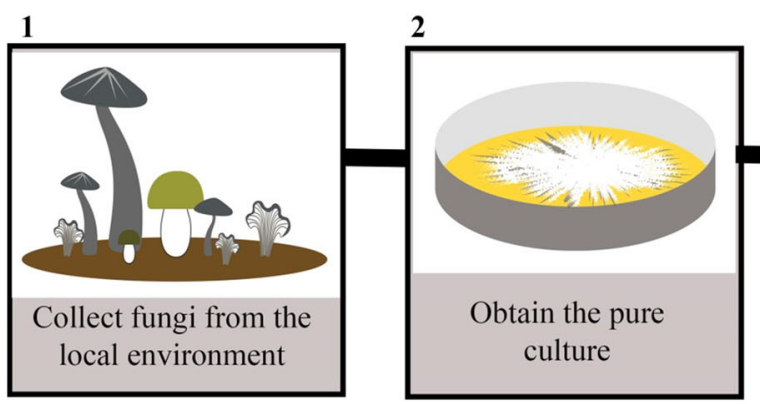

5

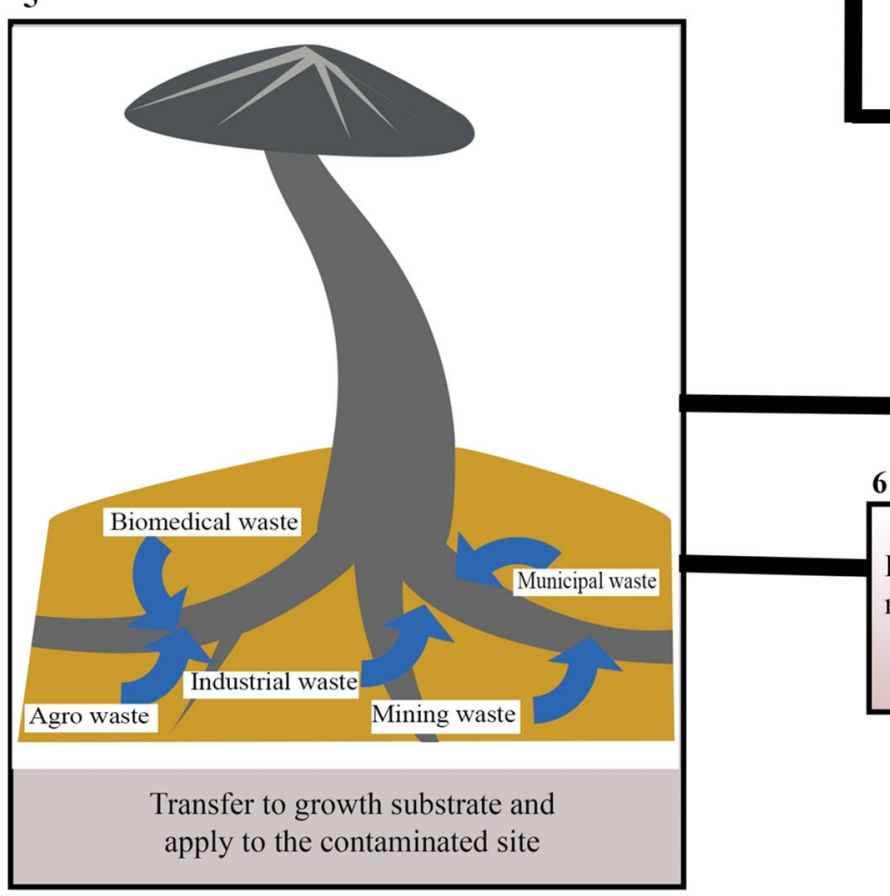

3

Bench scale testing for suitable enzymes production, Absorption ability

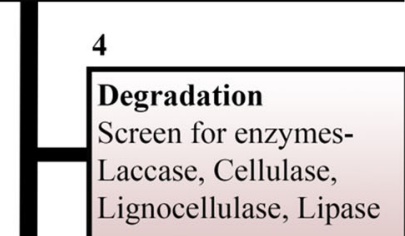

Ecofriendly, non toxic and reutilizable by-products 
Other conventional strategies for the decontamination of polluted sites, such as the use of chemicals or through incineration, are comparatively less effective than mycoremediation. The washing and extracting used in the chemical method only shifts the contaminant from one place to another (Valentín et al. 2013); likewise, incineration can be highly energy intensive, not cost effective, and has the potential to cause harm through the formation of dioxides (Shibamoto et al. 2007).

There are ongoing studies to test whether edible mushrooms could be used first for mycoremediation and then for human consumption. In one study, for example, Pleurotus pulmonarius was successfully tested for cleaning crude oil (Olusola and Anslem 2010); in another study, Agaricus bisporus and Lactarius piperatus were successfully used for the biosorption of $\mathrm{Cd}^{2+}$ under laboratory conditions (Nagy et al. 2013). However, these mycoremediation processes remain impractical due to the fact that the absorption of pollutants, such as heavy metals might render the edible part of the mushrooms highly contaminated (Kulshreshtha et al. 2014). However, if means of utilizing mushrooms with anti-mutagenic and anti-genotoxic abilities could be developed (Mendez-Espinoza et al. 2013), then it would be possible to simultaneously reduce the toxicity of the pollutants, clean the lands deemed unfit for agriculture, and produce crops or animal feed.

Some fungi not being able to grow in the presence of the natural microbiota of the contaminated site and the need to have precise understanding the nutrient requirements of the fungus for it to thrive in the contaminated site might be some small setbacks for this technology. In comparison to the alternative methods, these problems are negligible. And they can be readily solved with improved in-situ and exsitu trails.

The lack of more comprehensive information about degradation mechanisms and the enzymes involved in the pollutant degradation by different fungal species hinders the possible use of them in mycoremediation in a more specific manner. More in depth studies should be done to recognize the oxidative enzymes, organic acids and chelators excreted by fungi during the mineralization and degradation process. Another way to increase efficiency of mycoremediation is using large scale studies to verify the results of the bench scale studies that would provide insights in to efficient use of the existing species of fungi and identifying new species suitable for bioremediation. More resilient fungal species adapted to harsh environmental conditions should be tested for their adaptability as bioremediaters as they are more physiologically active. For example in some areas of the world, the soil is high in salt concentration due to inefficient drainage and this makes the ecosystems in these areas very simple and fragile. To make these areas more agriculturally feasible, halophilic or halotolerant fungi can be used use to clean up the soil of the excessive salt. Up until now most of the studies done regarding halophilic fungi only focus on the characterization. Fungi such as Aspergillus penicillioides, Gymnascella marismortui, Cladosporium cladosporioides and Penicillium westlingii are well characterized as halophilic but their potential use as soil mycoremediators should be done with more focused studies specifically designed to check their salt remediation ability (Zhang and Wei 2017).

By studying the genes related to enzyme degradation, mineralization, absorbance and adaptability to extreme environmental conditions, transgenic fungi can be produced with superior mycoremediation capabilities. However, since the remediation capacity of naturally occurring fungi is still very much understudied, isolating and screening suitable fungi from their natural habitats should carried out in order to reclaim polluted sites and to sustain a better environment for future generations.

\section{Mycofumigation using the genus Muscodor}

Mycofumigation is the use of antimicrobial volatiles produced by fungi for the control of other organisms (Stinson et al. 2003). The prototypes of the mycofumigant agents belong to strains of the genus Muscodor, on which most of the activities in research and development in this area have so far been focused. Muscodor species are endophytes that belong to the order Xylariales (Ezra et al. 2004; González et al. 2009; Kudalkar et al. 2012; Suwannarach et al. 2013a; Wijayawardene et al. 2018; Chen et al. 2019), but the taxonomic and phylogenetic position of the genus remains unsettled (Daranagama et al. 2018; Wendt et al. 2018). Muscodor cultures do not sporulate, their sexual state is as yet unknown, and their most salient common feature is their ability to produce volatile organic compounds. All strains of this genus can inhibit and kill other fungi, including human and plant pathogenic species, as well as bacteria and insects, through the production of a mixture of volatile organic compounds. These mixtures are primarily composed of various small molecular weight alcohols, organic acids, esters, ketones, and several aromatic hydrocarbons as determined by gas chromatographymass spectrometry. The mixture of VOCs produced from Muscodor species has been reported to be antimicrobial volatiles (for a selection see compounds 66-72 in Fig. 40; for original literature see, Atmosukarto et al. 2005; Kudalkar et al. 2012; Mercier and Jiménez 2004; Mitchell et al. 2008; Ramin et al. 2005; Siri-Udom et al. 2017; Suwannarach et al. 2013a, 2015a, 2017; Worapong et al. 2001; Zhang et al. 2010b). Studies by Alpha et al. (2015) and Hutchings et al. (2017) suggested that VOC produced by Muscodor may act in the bacterial DNA damage through inducing DNA alkylation and DNA methylation 
processes; however, the active ingredients yet remain to be identified. If such biological activities would also be observed in eukaryotes, including mammalian cells, this might constitute a future challenge for the registration of products based on the respective strains because of their mutagenic potential.

The most advanced project of this kind has focused on Muscodor albus (strain 620), originally isolated from Cinnamomum zeylanicum. This strain was shown to be an effective biofumigant capable of controlling lemon decay caused by Penicillium digitatum; lemon sour rot caused by Geotrichum citri-aurantii; apple decay caused by Penicillium expansum and Botrytis cinerea; as well as peach brown rot caused by Monilinia fructicola (Mercier and Jiménez 2004; Mercier and Smilanick 2005). The strain also can control grain smut fungi (Goates and Mercier 2009) and act as an insecticide against potato tuber moths (Lacey and Neven 2006). Another strain, Muscodor suthepensis (Fig. 41a), isolated from Cinnamomum bejolghota in Thailand, has potential application as a biofumigant for controlling blue mold (Penicillium digitatum) decay of the tangerine fruit (Fig. 42a) (Suwannarach et al. 2015a, b).

Muscodor inoculum also has the potential to replace methyl bromide (a toxic chemical fumigant and pesticide) fumigation in controlling soil-borne plant diseases. Muscodor albus (strain MFC2), isolated from Myristica

Fig. 40 Bioactive compounds reported from Muscodor species

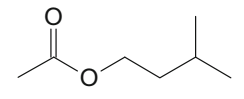

3-Methylbutyl acetate (66)
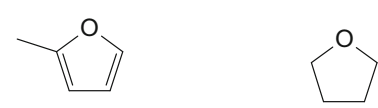

2-Methylfuran (69)

Tetrahydrofuran (70)

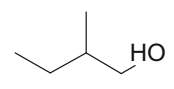

2-Methylbutan-1-ol (67)

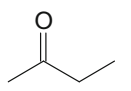

2-Butanone (71) fragrans, has the potential to control kale root rot disease caused by Pythium ultimum (Worapong and Strobel 2009). Muscodor albus (strain 620) and M. roseus (isolated from Grevillea pteridifolia) showed the ability to control disease severity caused by a variety of fungal and oomycete plant pathogens, e.g. Aphanomyces cochlioides, Rhizoctonia solani, Pythium ultimum and Verticillium dahliae (Stinson et al. 2003; Mercier and Jiménez 2009). Muscodor roseus also has nematicidal and nematostatic application against four plant-parasitic nematode species (Meloidogyne chitwoodi, M. hapla, Paratrichodorus allius, and P. penetrans) found on economically important vegetable crops (Riga et al. 2008). Muscodor cinnamomi (Fig. 41b) completely controlled damping-off symptoms on plant seedlings and Rhizoctonia-root rot in tomato plants, (Fig. 42c) (Suwannarach et al. 2012, 2015b). Muscodor heveae isolated from rubber trees (Hevea brasiliensis) is effective at controlling white root rot disease in rubber trees caused by Rigidoporus microporus (Siri-Udom et al. 2017).

The application of Muscodor in the control of soil-borne disease might be of concern due to the phytotoxic activity which depends on the mixture of volatile organic compounds and type of plant (Suwannarach et al. 2012; SiriUdom et al. 2017). Suwannarach et al. (2017) suggested that $M$. cinnamomi could be used in fumigation with an active culture of a biological control agent to reduce the number of microorganisms present on eggshell surfaces
Fig. 41 Colonies of Muscodor suthepensis (a) and $M$. cinnamomi (b) on potato dextrose agar at $25^{\circ} \mathrm{C}$ after 12 days. Scale bar $=1 \mathrm{~cm}$
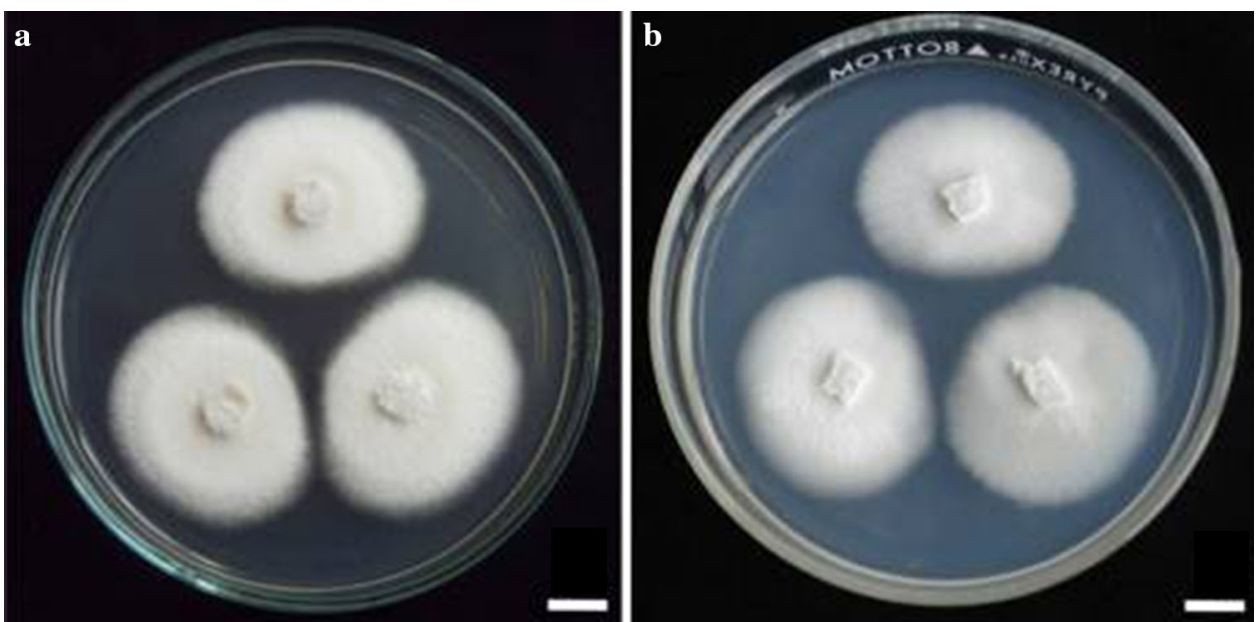


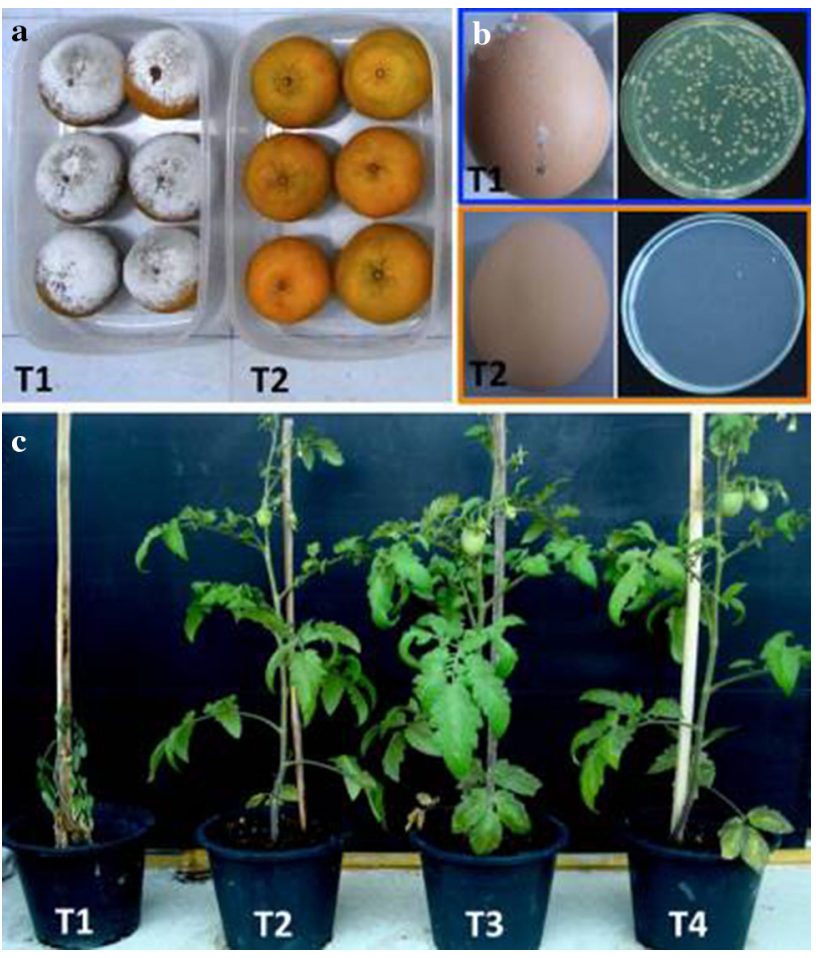

Fig. 42 Mycofumigation by Muscodor species. a Control of blue mold decay of tangerine fruit at 15 days of storage after $24 \mathrm{~h}$ fumigation by Muscodor suthepensis. b Eggs and microorganism on eggshell surfaces at 3 days of storage after $24 \mathrm{~h}$ fumigation with M. cinnamomi. c Control of tomato root rot disease by M. cinnamomi at 4 months after planting. $T 1$ infected control experiment, $T 2$ Muscodor experiment, T3 M. cinnamomi $+R$. solani experiment, T4 non-infected control experiment

without producing adverse effects on egg quality (Fig. 42b). Muscodor mycofumigants, through the use of the cereal grain inoculum, are being developed to control fruit decay and soil-borne diseases (Mercier and Smilanick 2005; Suwannarach et al. 2015a, b). Currently, the WAG $\mathrm{BAG}^{\circledR}$ Waste Kit, produced by M. albus, is a commercially available product for human waste disposal for application in aeronautics and by the military, which is regarded as non-toxic, biodegradable, and therefore environmentally friendly (Phillips et al. 2010).

Several other endophytic fungi, e.g. Gliocladium spp. Hypoxylon spp., Nodulisporium spp., Phomopsis spp., and Oxyporus latemarginatus, have been reported to produce volatile compounds that control fruit decay (Lee et al. 2009a; Park et al. 2010; Tomsheck et al. 2010; Singh et al. 2011; Suwannarach et al. 2013b). However, the identification of volatiles is often tentative, because no matching factors for GC/MS analytics have been given, and further effort on the characterization of the active principles are needed (cf. Helaly et al. 2018b). One way to safely identify the active components and assess their risk would be through total synthesis and subsequent biological testing in a manner similar to that in Wang et al. (2018b).

\section{Biomass to biofuel: Unmasking the potential of lesser-known fungi}

The conversion of lignocellulosic biomass (grasses, seaweeds, woody plants) to biofuel involves three phases: pretreatment, saccharification, and fermentation (Himmel et al. 2007). Lignocellulosic biomass is primarily made up of lignin, cellulose, and hemicellulose. Lignin, which imparts structural rigidity and integrity to plants, is a heterogeneous phenolic polymer with a variety of ether and carbon-carbon linkages. The delignification of biomass is necessary to expose the cellulose and hemicellulose polymers for their subsequent enzymatic hydrolysis into monomeric sugars (saccharification). Lignin's recalcitrance to depolymerization has prevented the cost-effective conversion of lignocellulosic biomass to sugars, and in some ways has undermined the viability of biorefinery operations. While the ability of a few white-rot fungi (basidiomycetes) to delignify lignocellulosic biomass has been explored in pre-treatment strategies (Tian et al. 2012; López-Abelairas et al. 2013), and cellulases and hemicellulases of Trichoderma reesei and Aspergillus niger (filamentous ascomycetes) have been extensively utilized for saccharification (de Souza et al. 2011; Keshavarz and Khalesi 2016), the emphasis on selected fungi has left the full potential of this vast kingdom largely untapped. By drawing attention to examples of less-studied fungi from unusual ecological niches, we highlight the payoffs from such initiatives to broaden the biocatalyst arsenal and to increase the prospects for new pre-treatment and saccharification enzyme cocktails and methods (Fig. 43).

The harsh physicochemical pre-treatment of lignocellulosic biomass often involves milling, as well as treatment with acid or alkali at high temperatures (Himmel et al. 2007; Wilson 2009; Wi et al. 2015). An alternative pretreatment entails the use of ionic liquids (e.g., 1-ethyl-3methylimidazolium acetate) to dissolve the lignocellulosic biomass and decrease lignin interference (Swatloski et al. 2002; Dadi et al. 2006; Wahlström and Suurnäkki 2015). Thus, ionic liquids afford a one-pot pre-treatment with saccharification (Shi et al. 2013), and are expected to increase the yield of sugars for fermentation. However, because ionic liquids inhibit the cellulolytic enzymes (Elgharbawy et al. 2016), ionic liquid-tolerant saccharification enzymes are necessary. Indeed, ionic liquid-tolerant endoglucanases, cellobiosidases, and $\beta$-glucosidases (the cellulolytic trio) from thermophilic/halophilic bacteria and archaea have been identified (Datta et al. 2010; Zhang et al. 2011b; Ilmberger et al. 2012; Park et al. 2012; Gladden 
Fig. 43 Novel roles for fungi in biofuel production. Adapted in part with permission from Ribeiro et al. (2016)

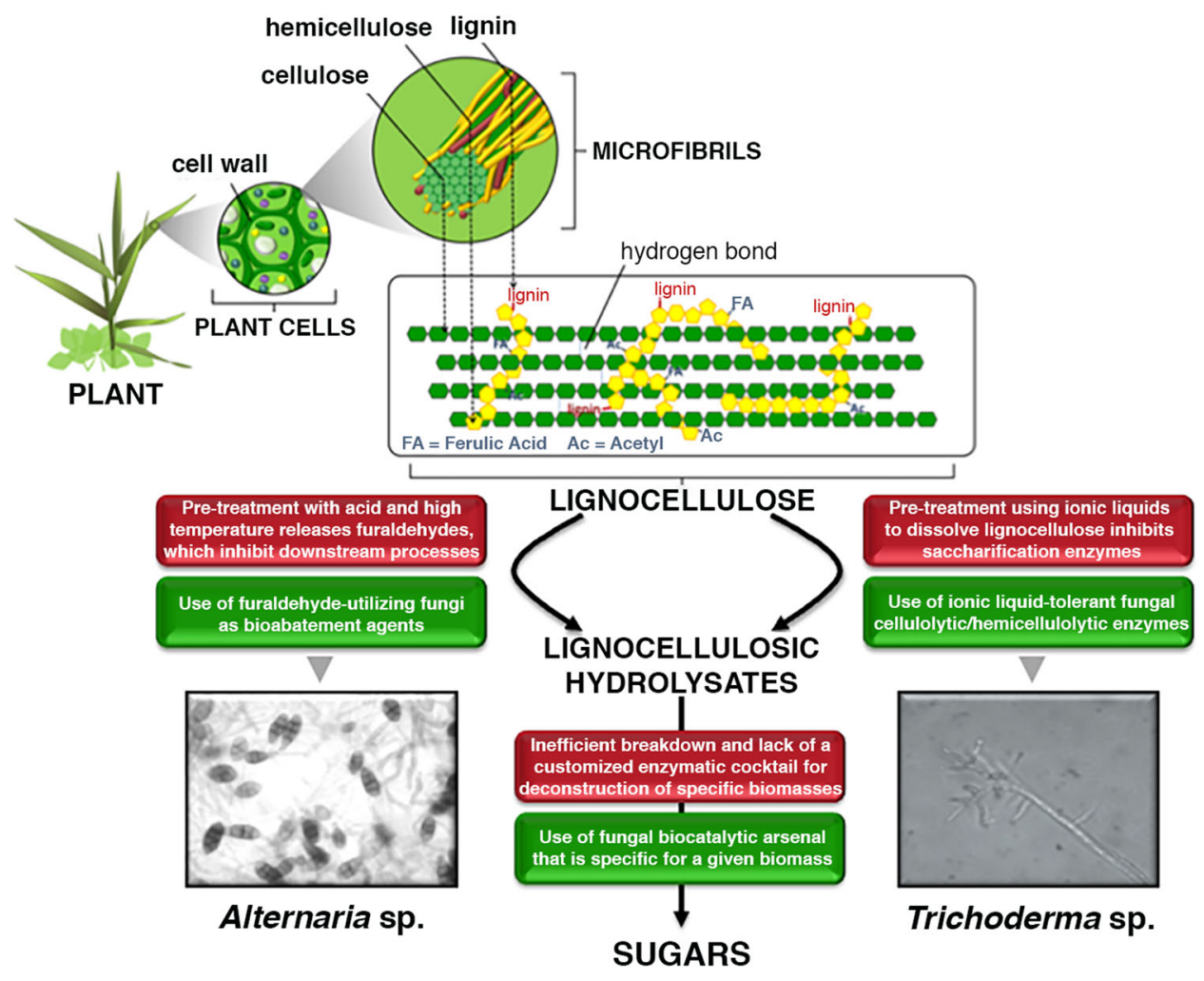

et al. 2014). For ionic liquid-tolerant hemi-cellulolytic enzymes, we directed our bio-prospecting efforts to marine-derived endophytic fungi, which have gainfully leveraged millions of years of co-evolution with marine plants/ algae to dominate the host microbiome and create a powerful catalytic repertoire that permits them to function as primary degraders of lignocellulosic biomass. We demonstrated that a mesophilic Trichoderma harzianum-isolated as an endosymbiont of the brown seaweed Sargassum wightii-produces ionic liquid-tolerant $\beta$-xylosidase, an enzyme needed for hemicellulose breakdown (Sengupta et al. 2017). Considering the structural difference in the xylans of brown seaweed and red/green algae growing in salt-rich habitats (Kloareg and Quatrano 1988), targeted bio-prospecting of associated endophytes should uncover ionic liquid-tolerant enzymes for lignocellulosic biomass deconstruction and saccharification.

The biological pretreatment of lignocellulosic biomass with white-rot fungi has been investigated owing to the fact that these organisms secrete lignin-degrading peroxidases and laccases (López-Abelairas et al. 2013; Yang et al. 2013b). While generic wood-rot basidiomycetes are useful in this regard, the idea that endophytic fungi (ascomycetes) isolated from a specific plant/tree might be evolutionarily fine-tuned for the deconstruction of its host biomass is supported by recent studies. For example, Ulocladium sp. and Hormonema sp., which are laccase-producing endophytes isolated from eucalyptus trees, were superior delignification agents relative to Trametes sp., an established laccase producer (Martín-Sampedro et al. 2015). In another example, Pestalotiopsis sp. was isolated from a mangrove (Arfi et al. 2013), and wood chips from Rhizophora stylosa mangrove trees were used to support the growth of this fungus. A proteomic analysis of this Pestalotiopsis sp. secretome revealed that $40 \%$ and $15 \%$ corresponded to glycosyl hydrolases and lignolytic enzymes, respectively. Endophytes isolated from such targeted bioprospecting are excellent tools for the deconstruction of their plant hosts, especially when used in conjunction with typical pretreatment methods that can now be performed at lower alkali/acid levels or temperatures. Second-generation variants, in which the aforementioned endophytes are genetically manipulated to overexpress specific enzymes (e.g., laccases) relative to the parental strain, will be worth developing. Transcriptome analysis of endophytic fungi capable of surviving as saprotrophs in abscised plant organs (Reddy et al. 2016; Guerreiro et al. 2018) would help to identify candidate cell wall polysaccharide-degrading enzymes that merit up-regulation in fungi that will be customized as pre-treatment agents. It would be instructive to investigate whether different genera of endophytic fungi exhibiting a biphasic life 
style have conserved a specific suite of enzymes for biomass degradation, as was discovered in the case of basidiomycetous fungi (Peng et al. 2018; López et al. 2018).

Pre-treatment with dilute acid at high temperature releases organic acids, phenolic derivatives and furaldehydes (furfural and 5-hydroxymethylfurfural) (Palmqvist and Hahn-Hägerdal 2000). The furaldehydes cause DNA damage, inactivate glycolytic enzymes, and inhibit downstream saccharification and fermentation, thus reducing the efficiency of biomass utilization (Caspeta et al. 2015). Although these inhibitors could be removed by washing and alkali treatment, or by ion exchange (Almeida et al. 2009), such methods are expensive, inefficient, and, significantly, wash away fermentable sugars. Under these conditions, the use of microbes which can metabolize such inhibitors offers a bioabatement strategy (Suryanarayanan et al. 2017).

Because furaldehydes are the most abundant and common volatile organic compounds released during biomass burning, we postulated that fungi in forests experiencing episodic fires can tolerate furfural and 5-hydroxymethylfurfural. Indeed, these two furaldehydes could be utilized by both endophytic and litter fungi from these forests, including those belonging to different taxonomic orders (Govinda Rajulu et al. 2014). These findings are consistent with those demonstrating the ability of Coniochaeta ligniaria (López et al. 2004), A. niger and T. reesei (Rumbold et al. 2009), and Amorphotheca resinae ZN1 (Zhang et al. 2010a) to use furfural or 5-hydroxymethylfurfural for growth. Using any of these fungi as bioabatement agents, however, will require construction of sugar-transport mutants that will minimize utilization of sugars and maximize consumption of furaldehydes.

Some of the currently used saccharification enzymes from Trichoderma reesei and Aspergillus niger exhibit low activity under industrial conditions (Druzhinina and Kubicek 2017), motivating the search for alternatives that are better suited for a specific biomass. Upon identification of an abundant biomass (in a given locale) for use as the main feedstock for biofuel production, the resident endophytes should be isolated and characterized for their delignification and saccharification capabilities. For example, Talaromyces borbonicus, a new species found in the naturally degrading biomass of Arundo donax (a tall cane), was sequenced and found to use $4 \%$ of its genome to code for 396 enzymes, all of which were linked to the breakdown, modification, or synthesis of glycosidic bonds (Varriale et al. 2018). In addition to A. donax deconstruction, this new palette of catalysts (once validated) will add to a growing inventory of saccharification enzymes. It is also useful to explore newer classes of enzymes, as exemplified by the fungal lytic polysaccharide mono-oxygenases, which revealed a novel oxidative (rather than hydrolytic) route to polysaccharide degradation (Couturier et al. 2018). These lytic polysaccharide mono-oxygenases (together with expansins and swollenins, which help loosen the cellulose microfibrils) are grouped under non-hydrolytic cellulose active proteins that collectively enhance the activity of cellulases in biomass hydrolysis (Ekwe et al. 2013). In light of these early successes with fungal nonhydrolytic cellulose active proteins (Moncalro and Filho 2017; Santos et al. 2017), it is essential to screen different ecological groups of fungi for superior variants.

New lessons have emerged from studying fungi not deemed model platforms for biofuel production. For instance, the anaerobic gut fungi of herbivores (e.g., goats, horses), which extract nutrients from seemingly intractable foliage, have many biomass-degrading enzymes that permit the utilization of a broad range of substrates (Solomon et al. 2016; Haitjema et al. 2017). Importantly, the synergy among carbohydrases in Neocallimastigota members (e.g., Piromyces, Neocallimastix) is the basis for the superior biomass-degradation capabilities of the herbivore gut. In another parallel, the fungus-cultivating termite symbiosis complex exemplifies a remarkable cooperation among different microbes for lignocellulosic biomass utilization ( $\mathrm{Li}$ et al. 2017). Within a colony, young termites use their gut microbiome to degrade lignocellulosic biomass, most notably the typically refractory lignin side-chains, and use their lignocellulosic biomass remnantrich faeces to build a fungal comb. The fungal microbiome in the comb cleaves lignocellulosic biomass polysaccharides and utilizes only xylose. The oligosaccharides in the comb sustain the older termites, which forage and transport plant material to the colony. This step-wise anaerobic and aerobic tandem deconstruction of lignocellulosic biomass occurs first within the gut of young termites and then in the fungal comb. This accounts for the comparatively faster pace with which termites degrade woody biomass when compared with herbivores.

While enzymes in model fungi (e.g., Trichoderma) have fostered advances for biofuel production, the two instances described above (Solomon et al. 2016; Li et al. 2017) demonstrate that lignocellulosic biomass deconstruction efficiency is due to synergy rather the catalytic arsenal per se; therefore, mimicking such consortia for industrial applications will be profitable. Thus, it would be worthwhile to screen the biomass-degrading ability of consortia of specific plant litter fungi at defined intervals during deconstruction, as different fungal species may contribute to different stages in the sequential breakdown (Vořrišsová and Baldrian 2013). Indeed, such a temporal orchestration of biomass degrading enzymes has also been reported for a bacterial consortium growing on sugarcane bagasse (Jiménez et al. 2018). 
Fungi are the primary degraders of plant biomass. Due to the complex structure of plant biomass and the longstanding interaction of fungi with plants (Lange et al. 2018), fungi have evolved a wide variety of biomass-deconstruction enzymes. However, since only a few fungal species have thus far been harnessed for their lignocellulolytic potential, it is essential to mine aerobic and anaerobic fungi from less-explored habitats [e.g., biogas plants (Young et al. 2018)]. Also, while the production of biomass-degrading enzymes by fungi is tightly controlled, the regulatory mechanisms are not highly conserved, as might be expected based on the diversity of ecological niches and lifestyles exhibited by fungi (Benocci et al. 2017). Thus, it is important to conduct omics studies on fungi from different habitats with varying lifestyles (saprobic, symbiotic and parasitic) in order to develop superior enzyme cocktails, or tailor pre-treatment agents. The finding that a single base pair difference among Trichoderma species could affect the expression and catalytic performance of biomass-degrading enzymes (Horta et al. 2018) affirms the need for a firm understanding of the underlying mechanisms for controlling gene expression.

\section{Packed-bed bioreactor for mycomaterial production}

Solid-state bioreactor systems have generally been considered the lesser alternative to liquid culture bioreactors for scaled generation and extraction of target proteins from bacteria and yeasts. Liquid culture allows for more efficient dissipation of heat, homogenization of cultures, and incremental addition of feedstock. While liquid culture provides a high degree of functionality for product extraction, they are largely limited to the production of discrete hyphal pellets or tissue sheets, making solid-statefermentation bioreactors optimal for applications leveraging the three-dimensional structure of mycelium or modifying a solid substrate. Numerous solid-state bioreactor designs have been implemented in industry, but the details of their development and application are seldom reported due to their proprietary nature (Mitchell et al. 2010).

For 10 years, Ecovative Design (Green Island, NY, USA) has been manufacturing mushroom composite materials which harness the structure of mushroom mycelium to produce products for protective packaging, furniture componentry, and other goods. These products utilize the structure of mycelium - a tenacious combination of cell wall chitin-glucan matrices and filamentous inter-cellular crosslinking - to bind discrete lignocellulosic particles into mycelium composites of defined geometry (Islam et al. 2018) with sufficient compressive and flexural strength to withstand use in a variety of high stress applications. Initially, these mycelium products were manufactured in a
Type I (Mitchell et al. 2010) passively aerated molded tray incubation system, wherein the maximum dimensionality was governed by the limitations of passive metabolic heat and gas diffusion. Since 2016, Ecovative has invested in the development of an actively aerated solid-state bioreactor system designed to enable gas-exchange and heatdissipation within large masses of mycelium composite through forced aeration with conditioned air. The adoption of this large, solid-state bioreactor (coined the Bulk Bin Reactor; see Fig. 44) has enabled the production of large geometry structural products that were impossible to produce with tray-based passively aerated systems. In addition, the necessary asepsis required for the production of this material has been greatly reduced compared to the former tray-based system. The $0.7 \mathrm{~m}^{3}$ blocks produced by the Bulk Bin Reactor system can be cut using a horizontal band saw mill into billets as thin as $3 \mathrm{~cm}$ thick, yielding multiple units from a single bin, and opening a variety of product opportunities for affordable flat stock panels. This represents the first report of the development and application of a Type II solid-state bioreactor system for mycelium material production, including a summary account of engineering and biological considerations.

The physical system consists primarily of an air pretreatment system and a vessel including air distribution (Mueller 2018). Pre-treatment of the air is critical for controlling temperature, humidity, and gas concentrations. Air is introduced to the system through a coarse particulate filter for protection of the blower. Critically, the blower is capable of providing air at a range of pressures which enables not only passage through the loose substrate prior to growth, but passage through the myceliated material at the end of the process cycle when pressures are highest. From the blower, the air is cooled to a programmable temperature via an intercooler or fan ventilator. This allows the system to run in an environment with fluctuating external temperatures, and also controls for the variable amount of heat added by the fan, which may change depending on load. Temperature controlled air can then be split into a plurality of flows for the support of multiple vessels. Here, flow (vol/vol/min) is also measured within each vessel to ensure that the desired flow rate is achieved.

Air at temperature next enters a humidification chamber wherein it is bubbled through a column of water. The humidification chamber is designed with a depth and size such that it can provide sufficient moisture into the air to fully saturate it. Additionally, as the process of evaporating water into the air stream requires heat, a heater is implemented to add the energy required to continually humidify the air, even at very high flow rates. By varying this energy input, it is possible to control with precision the humidity level during steady state operation. The humidification chamber (and all parts of the airflow pretreatment system) 
Fig. 44 a The bulk bin reactor system showing the air pretreatment system for air distribution and conditioning, and a bin incubation unit with a growing mycelium-

lignocellulose composite block; b example of a bulk bin reactor system mycelium-lignocellulose block, after extraction from the bin incubation unit and prior to post-processing; c light micrograph of epoxy thin section of mycelium-wood composite, showing a twodimensional section of the interparticle hyphal matrix; $\mathbf{d}$ panels cut from a bulk bin reactor system mycelium-lignocellulose block during post-processing, showing examples of a sealed surface (top), an applied laminate (middle), and an unfinished surface (bottom)
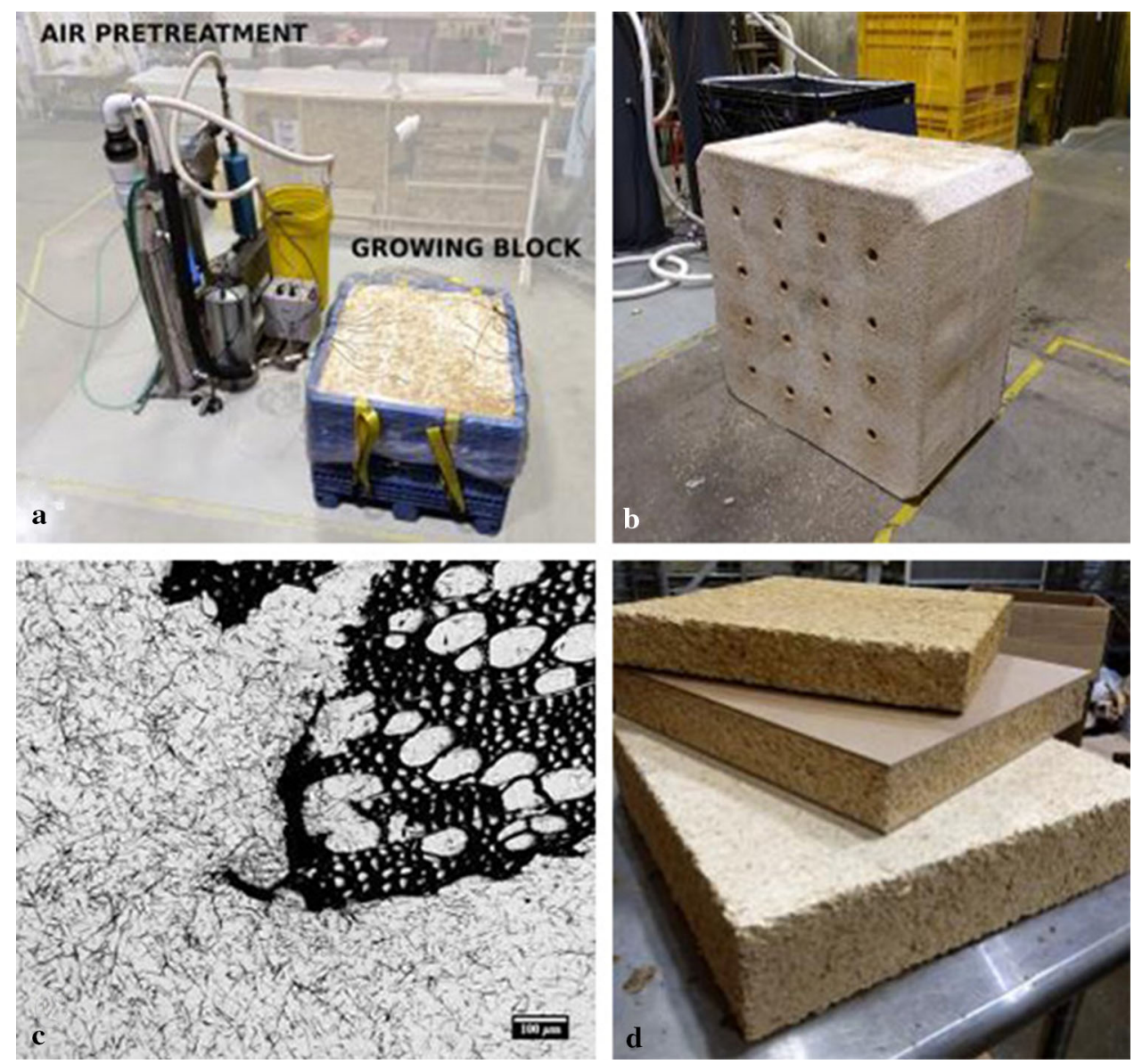

must be designed to handle the pressures which will be sustained at the end of the process when inter-particle colonization has reduced porosity.

The pre-treated air can be connected to a variety of vessel designs, from custom-molded shapes to generic blocks for later processing into panels. Design of the vessel depends on the nature of the substrate being used, particularly on its porosity, and on other variables such as the metabolic heat generated with the given combination of process parameters. In large vessels, an array of nozzles is used, each providing equal flow for the generation of uniform materials. The nozzles used are specially designed in order to provide uniform back pressure against free flow, therefore maintaining even flow rates through each nozzle and minimizing effects of random porosity variations in the material. The cross-sectional area of the nozzle and expansion area is selected such that even with partial blockage, the remaining cross-sectional area is still greater than the cross-sectional area of the flow restriction zone. This minimizes flow variation between nozzles caused by the random orientation of the chips.

It is important to note here that if $\mathrm{P}_{\mathrm{D}_{-} \mathrm{S}}+\mathrm{P}_{\mathrm{D}_{-} \mathrm{G}}$ exceeds the force of gravity on the substrate, the growing material will lift, opening low resistance air-flow channels that will bypass the material and increase gas-exchange heterogeneity. This is colloquially termed "burping", and whether it occurs in operation is a combined function of the porosity of the substrate; the density of inter-particle colonization; the air flow rate required; and the density of the substrateall of which combine to dictate the burping back-pressure.

One critical dimension of the vessel is its height. If aeration is introduced on a single side, for example on the bottom of a rectangular open top vessel, and if the vessel is sufficiently large in its length and width dimensions that heat loss through the walls cannot be considered for the central material, then its core is essentially a one dimensional thermodynamic and fluid dynamic system. In such a system, with heat being generated by each successive unit layer of material, the delta between the temperature of the material at the bottom of the vessel $\left(\mathrm{T}_{\mathrm{bot}}\right)$ and at the top $\left(\mathrm{T}_{\text {top }}\right)$ will be directly correlated with the height of the vessel. By the same logic, it is true that there will always be a temperature difference between the bottom and top of the vessel, so long as the material is generating heat and being cooled by aeration. It is important that the air flow rate, metabolic conditions, energy availability of the substrate, organism selected for growth, and height of the vessel all be selected in concert in order to ensure that the 
temperature delta between top and bottom is small enough that final properties of the top and bottom of the material are within the desired specifications.

A primary consideration for substrate and nutrition selection is the minimization of systemic sensitivity to contaminant organisms. The choice of a suitable primary substrate, composing the bulk of the raw material, is key to achieving stable and productive operation. Attributes to consider include density, porosity, nutritional availability, phytochemical composition, and cleanliness. From a holistic perspective, substrate composition-including spawn type, spawn rate, substrate species, supplemental nutrition, and temporal staging of supplemental nutritional inputs (i.e. pre- or post-priority effect)—is critical to the exclusion of contaminant organisms and to achieving a mechanically relevant stage of myceliation without strict aseptic control (Winiski 2015).

Strain selection involves a number of considerations, including ecological and metabolic habit, growth rate, hyphal morphology, and temperature sensitivity. Critically, the selected strain should have metabolic access to the recalcitrant lignocellulose in the base substrate while demonstrating growth rates that allow for exclusion of competitor species (Winiski 2015). Additionally, the selected strain should result in an inter-particle hyphal morphology that produces the desired mechanical properties. The organism selected must also be able to grow at a range of temperatures, but with generally similar growth rates at the range between $T_{\text {bot }}$ and $T_{\text {top }}$, thus enabling a uniform product.

One particular consideration in the production of mycelium materials with actively aerated static bioreactor systems involves the inherent conflict between the selection for inter-particle hyphal growth for material properties, and the resultant effect on inter-particle porosity and backpressure. Within this context, the morphology of interparticle growth governs air flow through the particle mass, ultimately affecting oxygen availability, heat dissipation, and thereby material heterogeneity, as a result of air channeling during inter-particle densification. For this reason, it is critical to understand the specific dynamics among the inter-particle hyphal network, mechanical properties, and system dynamics, in order to negotiate and optimize system operation and product properties. In this effort, simple and accessible techniques are employed for direct observation of inter-particle hyphal morphology by epoxy embedment and thin-sectioning, utilizing adaptations of well-established methodologies (Richardson et al. 1960). Open source image analysis software (Barry et al. 2015; Puspoki et al. 2016) is leveraged to perform quantitative analysis of the inter-particle hyphal matrix via light micrographs of the epoxy thin section (Fig. 44c). These methods and software applications provide accessible tools for extracting large quantities of morphological data with high sampling frequency (thousands of data-points on a scale of minutes), where manual measurement would be prohibitively laborious, and from which inter-particle morphological features such as hyphal length and area density, node density, inter-particle porosity, cell or strand thickness, and network orientation coherency and anisotropy can be related to system operation, environmental conditions, and material properties.

The described bulk bin reactor system is being leveraged to efficiently produce solid blocks of mycelium composite material (Table 20, mCore Panels and Blocks 2018), which is post-processed to panels of varying geometries using a horizontal band saw mill for applications including acoustic panels and wetland rafts, with further applications potentially envisaged for packaging, temporary signage and constructions, and insulation cores for doors. This material is manufactured within a 10-day processing time in $0.7 \mathrm{~m}^{3}$ blocks using the current bulk bin reactor paradigm, with plans for scaling to $>56 \mathrm{~m}^{3}$ with modification of existing lane composting system infrastructure with a projected cost of $<\$ 142 \mathrm{USD} / \mathrm{m}^{3}$ for operations above $42,000 \mathrm{~m}^{3}$ per year. For comparison, production costs for the Type I passively aerated molded tray system ranges from $\$ 350-700 \mathrm{USD} / \mathrm{m}^{3}$.

Previous research conducted by Pelletier et al. (2013) identified the potential application of mycelium composites produced with agricultural substrates as acoustic absorbers. In addition to the added benefits of a low-energy

Table 20 Material blends and properties

\begin{tabular}{lllll}
\hline Property & Standards & Hemp blend & Aspen chip & Aspen shaving \\
\hline Density $\left(\mathrm{kg} / \mathrm{m}^{3}\right)$ & & $102-129$ & 192 & 96 \\
Modulus of elasticity (MPa) & ASTM C165-07, D1621-16 & $0.89-1.72$ & $3.44-4.20$ & $0.27-0.48$ \\
$10 \%$ compressive strength (MPa) & ASTM C165-07, D1621-16 & $0.06-0.15$ & $0.20-0.41$ & $0.01-0.02$ \\
Flexural modulus (MPa) & C203-05a & & $10.34-15.85$ \\
Flexural strength (MPa) & C203-05a & $0.6-0.20$ & $0.07-0.11$ \\
Noise reduction coefficient (0-1) & ASTM C423 & Class A & 0.4 & 0.9 \\
Fire rating & ASTM E84 & & 0.65 \\
\hline
\end{tabular}


manufacturing process and biodegradability at the product's end-of-life stage, it was found that optimum performance occurred at the critical automotive road noise frequency of $1000 \mathrm{~Hz}$. Subsequently, testing was performed with panels produced within the bulk bin reactor paradigm with three substrate blends (Table 20), according to ASTM C423 using an IBM HVAL with a Norsonics NE840 real time analyser. The highest sound absorption was found with the aspen shaving blend, which boasted an NRC rating of 0.9 , thus demonstrating a Class A absorption coefficient and suggesting potential application for manufacture of low VOC, biodegradable, acoustic panels. Stateof-the-art acoustic panels are produced with polyurethane foam, with mineral wool and fiberglass providing lowVOC options, but there is a growing interest in development of natural alternatives due to human health and product end-of-life concerns (Kaamin 2017).

A critical property for acoustic panels for indoor applications is fire rating. Jones et al. (2017) reported meaningfully lower $\mathrm{CO} 2$ and $\mathrm{CO}$ release, along with reduced smoke density, for mycelium-rice hull composites, as compared to XPS by cone calorimeter. Additionally, fire performance can be improved by amendment with glass fines (Jones et al. 2018).

Constructed floating wetland panels represent another application opportunity for the mechanical and end-of-life properties of mycelium composites. Constructed floating wetlands are primarily utilized to improve wetland water or wastewater quality, though they are also used for a variety of other objectives, including habitat creation, shoreline protection, and landscape improvement. Floating wetlands are typically constructed from a variety of materials, including non-biodegradable materials such as extruded polystyrene, PVC pipes, and high-density polyethylene (Pavlineri et al. 2017). In this consideration, mycelium composite panels may provide a biodegradable option with the required mechanical and hydrophobic properties (Haneef et al. 2017). This application is being tested by the Green Futures Research and Design Lab at the University of Washington (Seattle, WA, USA), with mycelium composite panels currently in field deployment.

\section{Fungal degradation of plastics: $A$ hidden treasure for a greener environment}

In the modern plastic era, primary plastic production has increased to 335 million tonnes per year, with Asia accounting for $50 \%$ of the total worldwide production (PlasticsEurope 2017). According to a recent estimate, from 1950 to 2015 about 9150 million tonnes of primary plastics were produced, resulting in the accumulation of about 6945 million tonnes of plastic waste on the surface of the earth. Of the total waste generated, only a small percentage was recycled $(9 \%)$ and incinerated $(12 \%)$, while $79 \%$ amassed in landfills and other terrestrial and marine environments (Geyer et al. 2017).

The majority of petroleum-based plastics, such as polyethylene (PE), poly(ethylene terephthalate) (PET), polystyrenes (PS), polyurethanes (PUs), and polyvinyl chloride (PVC), are resistant to biodegradation, and therefore persist in the environment with various consequences (Wei and Zimmermann 2017). Biodegradable plastics are considered eco-friendly alternatives to petroleum-based non-biodegradable polymers. However, limited information is available regarding the exact mechanisms underlying the biodegradation process, the time scale of biodegradation, and the optimal environmental conditions required for their biodegradation (Yang et al. 2014a). Thus, the careless disposal and degradation of both biodegradable and synthetic plastics result in the accumulation of waste in terrestrial landfills and marine environments, and pose a serious threat to surrounding ecosystems (Andrady 2015). Nonetheless, numerous studies have reported that plastics are vulnerable to microbial attack (Barratt et al. 2003; Krasowska et al. 2012; Mathur and Prasad 2012; Zafar et al. 2013, 2014; Restrepo-Flórez et al. 2014; Khan et al. 2017).

The conservational and ecological roles of fungi are not only limited to energy or elemental cycling, but can also be used in the biodegradation of various types of plastics. Numerous studies have mentioned fungi as the predominant microorganisms responsible for the biodegradation of both bio- and synthetic- plastics (Barratt et al. 2003; Khan et al. 2017). According to the findings of one survey, the majority of plastic degrading fungi belong to the genera Aspergillus, Fusarium, Paecilomyces and Penicillium (Kim and Rhee 2003). The mechanical forces exerted by the fungi during growth, hyphal penetration into the plastic layer, as well as the simultaneous secretion of various enzymes and radicals, are all characterized as the fungal degradation of plastics (Moore et al. 2000). The mechanism of fungal degradation begins with the attachment of hyphal filaments to the surface of a plastic substrate. The hyphal tips then extend, using mechanical force to secrete enzymes and radicles that allow the penetration of the fungus into the substrate (see Fig. 45).

The next step is the absorption of small molecules, polymers, radicles, or atoms through the porous tips of the hyphae, followed by their transportation through the underlying plasma membrane (Moore et al. 2000; Khan et al. 2017). The enzymes are known to hydrolyze the polymeric substrate and in turn provide nutrients, which facilitates the growth of fungi or other microorganisms (Santerre et al. 1994; Lucas et al. 2008; Banerjee et al. 
2014). The various fungal species and the associated enzymes reported to degrade various types of plastics are listed in Table 21.

Most of the enzymes known for the degradation of plant polymers are able to depolymerize synthetic polymers, such as PE and PU, by hydrolyzing the ester bonds present in the polymer backbone. The surface area of polymers exposed to fungal enzyme attack has an advancing effect on biodegradation. Therefore, plastic particles in the range of 0.5 to $0.25 \mathrm{~mm}$ are most suitable for enzyme biodegradation. Although purified enzymes are known to breakdown the $\mathrm{C}-\mathrm{C}$ bond in polyvinyl chloride (PVC), the use of microorganisms is favored for the biodegradation of polymers. Moreover, the mixed cultivation of several strains is known to have a greater impact on biodegradation efficiencies than the use of single strains (Wei and Zimmermann 2017).

The overall process of plastic biodegradation is dependent on the presence of optimal environmental conditions to facilitate the growth of fungi and maximize the activities of the enzymes to depolymerize the polymeric materials. The biodegradation process is also affected by the physiochemical properties of the plastics, such as surface topology, molecular weight, hydrophobicity, and the degree of crystallinity (Manzur et al. 2004; Brueckner et al. 2008; Jenkins and Harrison 2008; Ronkvist et al. 2009; Restrepo-Flórez et al. 2014; Wei and Zimmermann 2017). Higher degrees of crystallinity strongly reduced the biodegradation of specific plastics. Aliphatic carbon chains are more easily biodegradable than aromatic polymers. The hydrophobicity of plastics makes the surface water repellant, reducing the success of the attachment, growth, and propagation of fungal hyphae, and thereby reducing the degree of plastic biodegradation. Similarly, abiotic factors such as environmental temperature; availability of oxygen; exposure to light or UV; and availability of radicles in the environment all affect the mechanisms of plastic biodegradation (Wei and Zimmermann 2017).

The diversity, zonal distribution, and niche partitioning of fungal strains in the environment also affect the biodegradation process. Furthermore, the amount and type of enzymes secreted by the fungal strain to digest the polymer also strongly affect the biodegradation process (Fig. 10). The secretion of specific types of enzymes or various types of enzymes by fungi to degrade polymers
Fig. 45 Fungal degradation of polyester polyurethane (PEU) films. a PEU film not exposed to fungal degradation (control); $\mathbf{b}$ d PEU films exposed to different Aspergillus spp. on malt extract agar medium incubated for 28 days in dark at $30{ }^{\circ} \mathrm{C}$. The extent of biodegradation differed for each strain. Fungal hyphal growth is observed on the PEU surface and some portions of the PEU films are degraded due to fungal activities
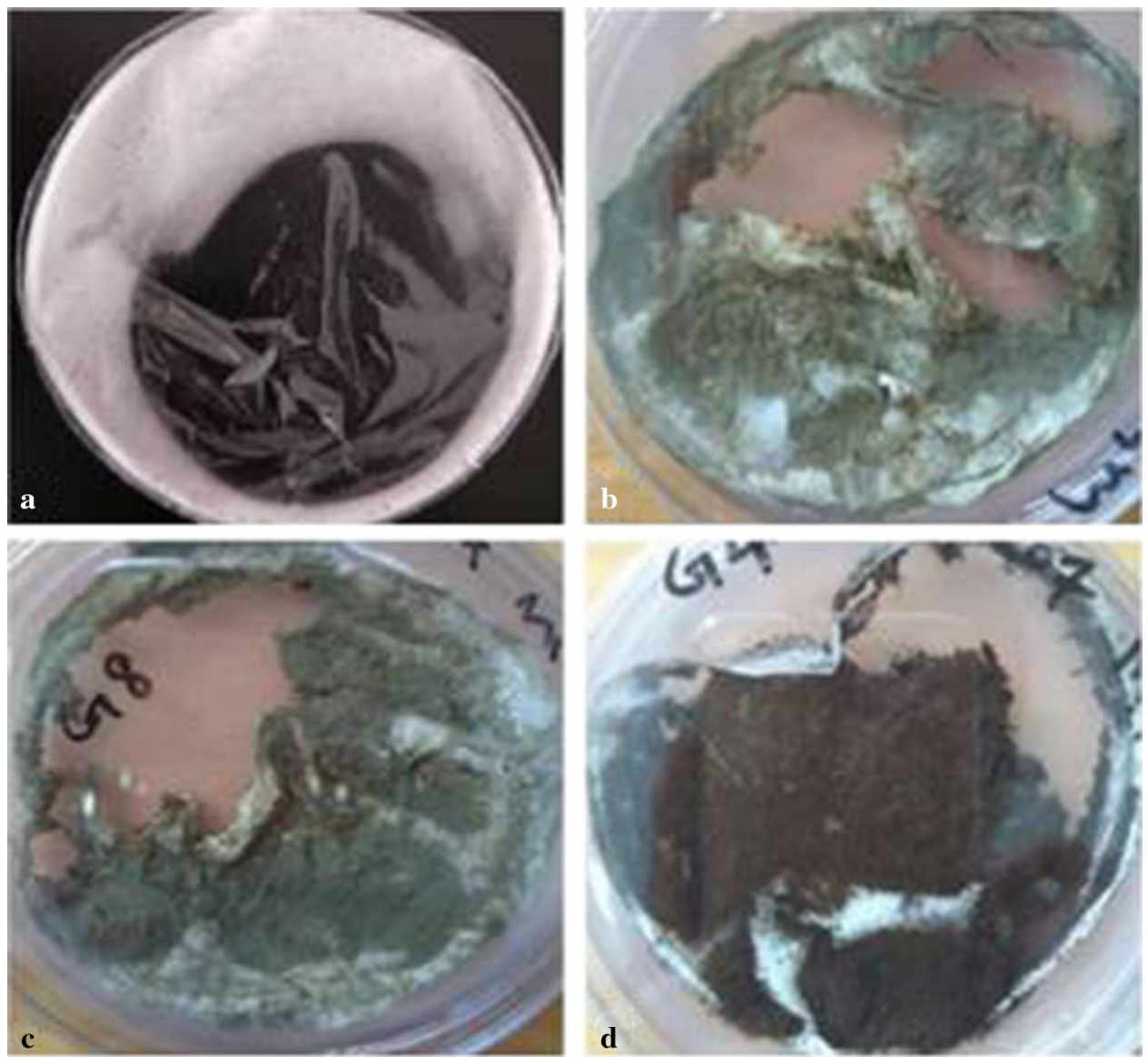
Table 21 List of fungal species with their enzymes responsible for biodegradation and types of plastics used

\begin{tabular}{|c|c|c|c|}
\hline Fungal strain & Enzyme & $\begin{array}{l}\text { Plastic } \\
\text { type }\end{array}$ & References \\
\hline Aspergillus flavus & Glucosidases & PCL & Tokiwa et al. (2009) \\
\hline Aspergillus niger & Catalase, protease & PCL & Tokiwa et al. (2009) \\
\hline Aspergillus terreus & Esterase, urethane hydrolase & PU & Boubendir (1993) \\
\hline Aspergillus tubingensis & Esterase, lipase & PU & Khan et al. (2017) \\
\hline Bipolaris (Cochliobolus) sp. & Laccase & PVC & Sumathi et al. (2016) \\
\hline Chaetomium globosum & Esterase, urethane hydrolase & PU & Boubendir (1993) \\
\hline Curvularia senegalensis & Polyurethanase & PU & Crabbe et al. (1994) \\
\hline Fusarium sp. & Cutinase & PCL & Shimao (2001) \\
\hline Pestalotiopsis microspora & Serine hydrolase & $\mathrm{PU}$ & Russell et al. (2011) \\
\hline Phanerochaete chrysosporium & Manganese peroxidase & $\mathrm{PE}$ & Shimao (2001) \\
\hline $\begin{array}{l}\text { Talaromyces funiculosus (formerly Penicillium } \\
\text { funiculosum) }\end{array}$ & Glucosidases & PHB & Tokiwa et al. (2009) \\
\hline Talaromyces pinophilus (formerly Penicillium pinophilum) & PHB-depolymeras & PHB & Panagiotidou et al. (2014) \\
\hline Trichoderma sp. & $\begin{array}{l}\text { Urease, protease, esterase and } \\
\text { laccase }\end{array}$ & PU & $\begin{array}{l}\text { Loredo-Treviño et al. } \\
\text { (2011) }\end{array}$ \\
\hline
\end{tabular}

Disclaimer: Some of the fungal species in the table are pathogenic and it is out of question to use them in biotechnological processes $P C L$ polycaprolactone, $P E$ polyethylene, $P H B$ polyhydroxybutyrate, $P U$ polyurethane, $P V C$ polyvinyl chloride

Fig. 46 Polyester polyurethane (PEU) films with fungal growth and signs of biodegradation on the surface. a PEU film not exposed to Aspergillus species; b fungal growth on the surface of PEU; c, $\mathbf{d}$ PEU films after the washing of fungal hyphae from the surface of the films
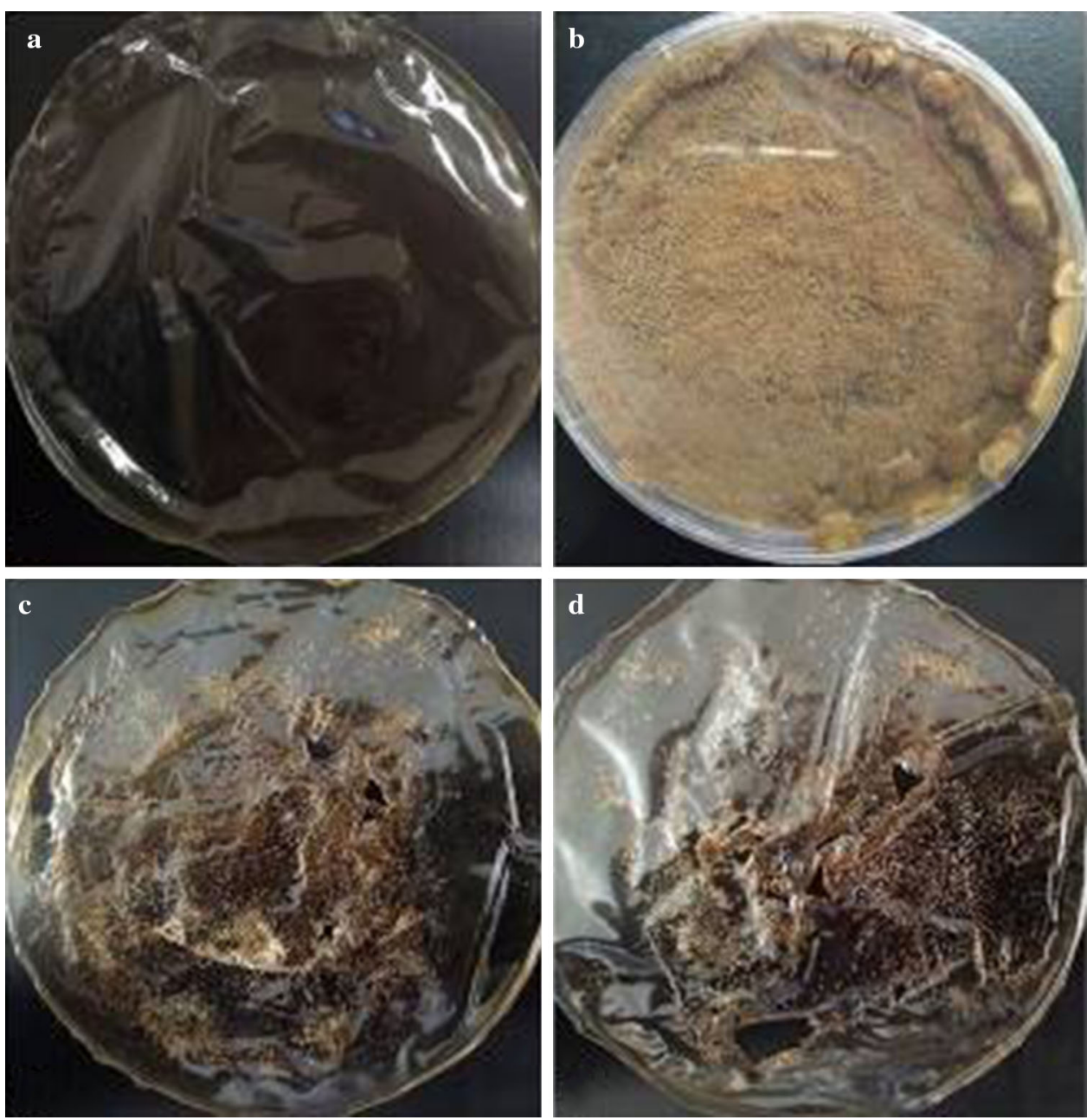
vary from one species to another, or even within the same species (Doi 1990; Howard 2012).

Currently, more than 100 fungal species are known to degrade different types of plastics, and many more are still in the process of being isolated and identified (Howard 2012). Most of the fungal strains discovered were studied for their enzyme biodegradation abilities at the laboratory scale. No efforts have yet been made to develop large scale or industrial scale bioreactors for specific types of plastic degrading fungi. Therefore, this research is of great importance for industrial development, as well as for future research purposes. Collaborations between chemists, biologists, engineers and physicists could result in the development of a large-scale bioreactor for plastic biodegradation utilizing fungi. At present, such processes are too expensive to be applied at large scale, and collecting and burning plastics under high heat remains more economical. However, once such bioreactors are successfully developed, new industries will emerge for both the degradation of various plastics as well as for the utilization of the degraded compounds in the manufacturing of new products. There is a need to properly collect disposed plastics and develop sophisticated large-scale bioreactors in which fungi could be used to degrade various types of plastics separately in controlled conditions using modern biotechnological techniques. Attention should be given to research and development on immobilized enzyme technologies with regard to fungal-degradation of plastics through enzymes. More knowledge is needed concerning the connection between specific-plastic types and specific fungal species, to determine the actual food web of various fungi. Integration of low-cost and easily degradable bioplastics in markets around the globe is important. The mechanisms of fungal degradation of plastics need to be clarified using isotopic marking of plastics. The structure and active sites of biodegrading enzymes of various fungi need to be determined, and methods to increase the catalytic degradation of polymers need to be developed.

\section{Polycyclic aromatic hydrocarbon degradation by basidiomycetes}

Polycyclic aromatic hydrocarbons are ubiquitous contaminants widely distributed in the environment, with suspected carcinogenic and mutagenic effects. The clean-up of polycyclic aromatic hydrocarbon contaminated sites is of great importance to protecting human health. Polycyclic aromatic hydrocarbons are organic compounds composed of two or more fused benzene rings which are formed during the combustion of organic molecules and their subsequent recombination (Haritash and Kaushik 2009).

Low molecular weight polycyclic aromatic hydrocarbons are composed of 2-3 aromatic rings. They are commonly found in petrogenic sources (e.g. gasoline, kerosene) and can be introduced to the aquatic environment through oil spillage and discharge from ships, tankers, motorboats, etc. Contamination can also come from municipal and urban runoff (Vieira et al. 2018). Polycyclic aromatic hydrocarbons of high molecular weight, composed of 4-6 aromatic rings (e.g. pyrene and benzo[a] pyrene), are often released into the environment through pyrogenic sources (Souza et al. 2015). Such polycyclic aromatic hydrocarbons are considered priority pollutants because they are recalcitrant in the soil, due to their high affinity and low water solubility (Rothermich et al. 2002).

The biodegradation of polycyclic aromatic hydrocarbon (Fig. 46) contaminants is a complex process which is highly dependent on the number of contaminants, natural conditions (e.g. temperature, humidity and climate), and presence of organisms consuming the available hydrocarbons. Polycyclic aromatic hydrocarbons tend to bind to soil components, thus becoming difficult to remove and degrade (Pandey et al. 2016). Hydrocarbons differ in their susceptibility to microbial attack, in which some of the high molecular weight polycyclic aromatic hydrocarbons may only be partially degraded or not degraded at all (Atlas and Bragg 2009).

Fungi can turn hydrocarbon contaminants into an energy source. In the hydrocarbon contaminated environment, those fungi capable of degrading hydrocarbon have better chance of surviving by utilizing this carbon source for growth (Fernandez-Luqueno et al. 2010). Some fungi, and in particular basidiomycetes, are efficient degraders of high molecular weight polycyclic aromatic hydrocarbons. In this way, fungi are unlike bacteria, which are better equipped for degrading smaller molecules (Peng et al. 2008).

The biodegradation of polycyclic aromatic hydrocarbons can be enhanced through extracellular oxidation. White rot fungi are the most efficient producers of oxidative extracellular enzymes. White-rot fungi are wood degrading basidiomycetes which produce special oxidases or enzymes that help in the degradation of lignin and other plant polymers. These enzymes can also degrade a variety of chemicals, including environmental pollutants (Crawford 2006) such as polycyclic aromatic hydrocarbon.

Successful polycyclic aromatic hydrocarbon biodegradation is brought about by different catabolic activities, such as metabolic capabilities, the induction of specific enzymes, and the presence of favourable organisms capable of degradation (Al-Hawash et al. 2018) under favourable conditions.

The degradation of polycyclic aromatic hydrocarbons can be affected by conditions such as temperature, oxygen, $\mathrm{pH}$, and nutrient availability. Temperatures can affect the physical and chemical composition of polycyclic aromatic hydrocarbons: at low temperatures, the degradation rate is 
slow due to the decreased enzymatic activities (Bisht et al. 2015). Polycyclic aromatic hydrocarbon degradation can actually occur at a wide range of temperatures according to medium: the maximum degradation was documented to be in the range of 30 to $40^{\circ} \mathrm{C}$ in soil environments, 20 to $30^{\circ}$ $\mathrm{C}$ in marine environments, and 15 to $20^{\circ} \mathrm{C}$ in fresh water environments (Al-Hawash et al. 2018). Oxygen concentration is also important in the biodegradation process. Biodegradation can be at its best in highly oxygenated environments. Though the degradation of polycyclic aromatic hydrocarbons can also occur at negligible rates under anaerobic environments (Haritash and Kaushik 2009), the process is not as rapid as under aerobic conditions ( $\mathrm{Gr}-$ ishchenkov et al. 2000). The $\mathrm{pH}$ value should also be taken into account in the successful degradation of polycyclic aromatic hydrocarbons, as it regulates the passage of small molecules and ions in the biological membrane and affects the catalytic reaction balance. In general, fungi would prefer nearly neutral $\mathrm{pH}$ to maximize their degradation activity, although they can also tolerate acidic conditions (Bonomo et al. 2001). Nutrients can also become a limiting factor in the process of biodegradation. The concentration as well as the ratio of certain nutrients such as carbon, nitrogen, iron and phosphorous can affect the growth and sporulation of hydrocarbon-degrading fungi (Zafra and Cortés-Espinosa 2015). The chemical composition of polycyclic aromatic hydrocarbons is yet another factor that can greatly affect the success of polycyclic aromatic hydrocarbon degradation. The higher the molecular weight, the more recalcitrant polycyclic aromatic hydrocarbons are in the environment, thus taking much longer to be degraded (Fedorak and Westlake 1981).

Basidiomycetes use a variety of mechanisms to completely degrade organic compounds such as hydrocarbons. In general, both oxidative and reductive reactions are required for the complete metabolism of hydrocarbon compounds. The first step in degradation occurs at the intra-cellular level in the form of oxidation and the integration of oxygen with the aid of the enzymatic catalysts peroxidases and oxygenates (Al-Hawash et al. 2018). Basidiomycetes secrete these enzymes to catalyse both the direct and indirect oxidation of chemicals. The enzymes, which are oxidoreductases, aid in the detoxification of toxic organic compounds through biochemical reactions, which in turn transform polycyclic aromatic hydrocarbons step by step into intermediates of the central intermediary metabolisms (such as the tricarboxylic acid cycle). The action of oxidoreductases renders the contaminants and xenobiotics into harmless compounds that can be easily degraded further (ITRC 2002). For instance, the ligninolytic fungus, Pleurotus ostreatus, can degrade phenanthrene into less harmful compounds, including 2,2'-diphenic acid (Fig. 47).
White-rot basidiomycetes produced non-specific extracellular ligninolytic enzymes consisting of three groups: lignin peroxidase (LiP), manganese-dependent peroxidases (MnP), and laccase (Hofrichter et al. 2001), all of which are essential for the transformation and mineralization of organic pollutants (Wang et al. 2009) such as polycyclic aromatic hydrocarbons. The genera Ganoderma (Agrawal et al. 2018), Armillaria (Hadibarata and Kristanti 2013), Coprinus (Li et al. 2009), Marasmiellus (Vieira et al. 2018), Pleurotus (Hadibarata and Teh 2014; Li et al. 2009), Pycnoporus (Munusamy et al. 2008) and Phanerochaete (Wang et al. 2009) were able to degrade polycyclic aromatic hydrocarbon moieties such as anthracene, pyrene, phenanthrene and benzo[a]pyrene (Table 22).

Many fungal species, and in particular white-rot basidiomycetes, have demonstrated the ability to degrade polycyclic aromatic hydrocarbons under laboratory conditions; however, these processes require further investigation in the field, and to date a limited number of studies have tested the efficacy of these fungi in the degradation of polycyclic aromatic hydrocarbons within typical environmental conditions. The placement of fungi into the environment requires further studies to evaluate their impact on other organisms. Intensive studies should also be conducted into analytical chemistry and genetic engineering tools which could help in successful bioremediation to increase fungal efficiency in degrading hydrocarbons and other recalcitrant contaminants. Furthermore, through the optimization of adsorption, bioavailability and mass transfer of polycyclic aromatic hydrocarbons, scientists could enhance the rate of bioremediation. This could provide one effective means of mitigating environmental pollution.

\section{Can fungi help modify the sustainable soil enhancer biochar?}

Biochar has achieved popularity as a sustainable soil enhancer. Biochar is derived from biomass such as leaves, forestry and agricultural residue, and animal manure (Spokas 2010; Shackley et al. 2012). This biomass is converted into a highly porous, carbonaceous product through a controlled pyrolysis process, namely thermochemical decomposition in the absence of oxygen (preventing combustion). In this way, it is different from charcoal. While charcoal produces energy, biochar is used in carbon sequestration, environmental management, and soil amendments (Lehmann and Joseph 2009; Warnock et al. 2007).

Biochar is added to poorly performing or contaminated soil as a means of increasing the recalcitrance of organic soil matter in a sustainable manner. Due to its polycondensed aromatic structures, the added biochar can remain 
Fig. 47 Proposed pathway for the degradation of phenanthrene by the ligninolytic fungus Pleurotus ostreatus (Bezalel et al. 1996; Aust et al. 2003; Gupte et al. 2016)

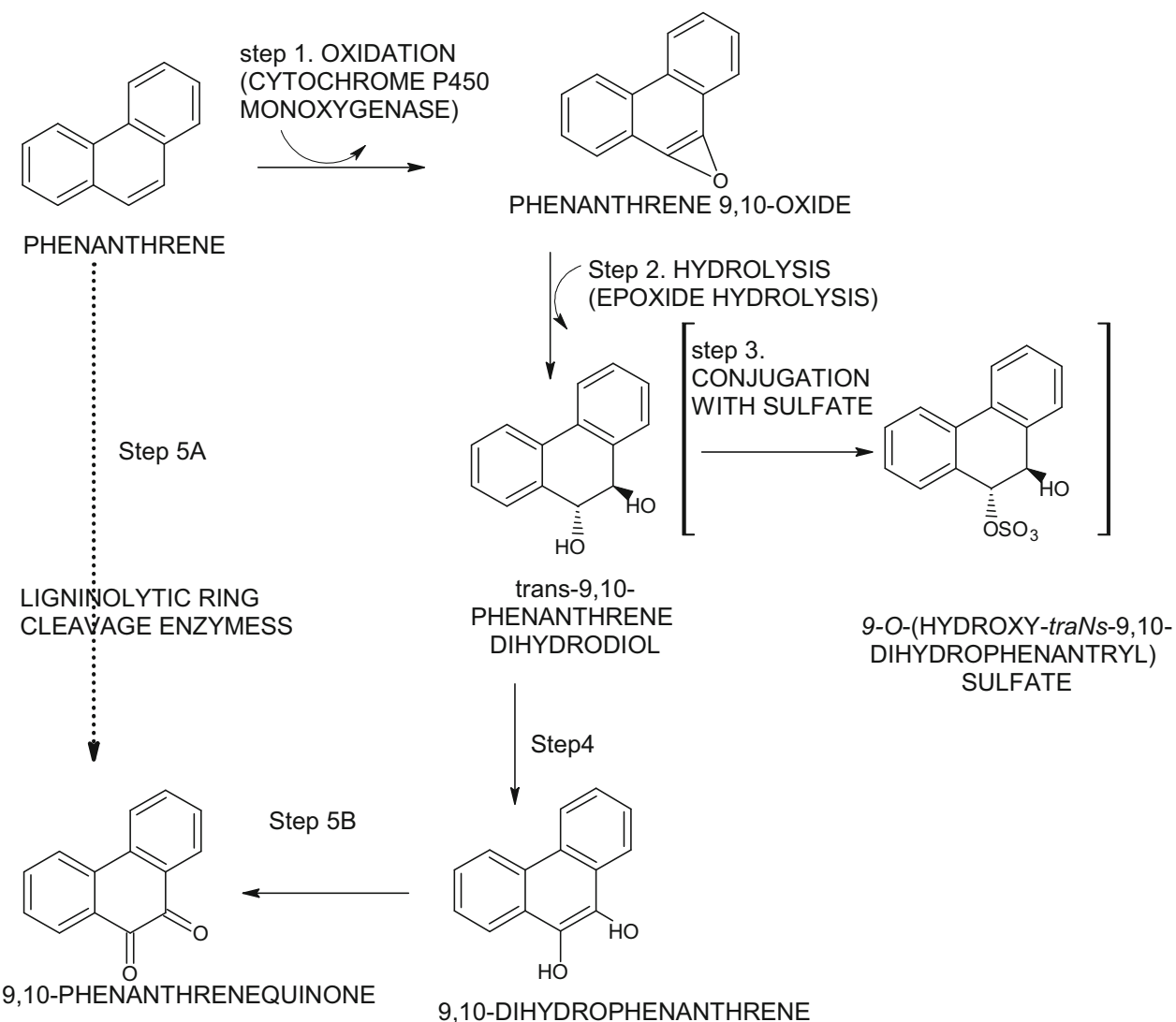

2, 2'-DIPHENIC ACID

in the soil for centuries to millennia, thus contributing higher levels of aromatic compounds to the soil over long periods of time. Important physicochemical properties for the evaluation of biochar utilized in soil have been defined: $\mathrm{pH}$, volatile compounds content, ash content, bulk density, water-holding capacity, pore diameter and volume, and specific surface area (Sohi et al. 2010). The origin of the biomass or feedstock and the pyrolysis conditions, therefore, are the key factors governing the physicochemical properties of biochar. Within the pyrolysis process, temperatures between 400 and $500{ }^{\circ} \mathrm{C}$ produce higher amounts of biochar (Gaunt and Lehmann 2008; Qambrani et al. 2017). While pyrolysis occurs more rapidly at high temperatures, a slowed pyrolysis process can produce a substantially higher quantity of biochar product. The yields of ash-free biochar were positively correlated with the cellulose, hemicellulose and lignin contents of feedstock; the biomass samples possessing higher lignin content produced higher amounts of biochar, and the samples of high ash content hampered biochar production (Sun et al. 2017).

When incorporated into soils, biochar exhibits a natural oxidation through the formation of functional groups. These groups are dependent on the temperature employed to produce the biochar. Thus, biochars are redox active and as such contribute to the reductive transformation of organic contaminants in soil by facilitating electron transfer from bulk chemical electron donors (e.g. phenolic moieties arising from the lignin in the original biomass) to the receiving organic compounds (e.g. quinone-hydroquinone moieties) and redox-active metals (e.g. iron, manganese, copper), reversibly donating and receiving up to $2 \mathrm{mmol}$ electrons per gram of biochar (Klüpfel et al. 2014). Biochar-mediated microbial electron shuttling has also been considered as an important process in soil 
Table 22 Degradation of polycyclic aromatic hydrocarbons by enzymes from basidiomycetes

\begin{tabular}{|c|c|c|c|c|}
\hline Enzyme & Basidiomycete strain & $\begin{array}{l}\text { Polycyclic aromatic hydrocarbon } \\
\text { moities }\end{array}$ & $\begin{array}{l}\text { Percentage of degradation } \\
(\%)\end{array}$ & References \\
\hline \multirow[t]{14}{*}{ Laccase } & \multirow[t]{2}{*}{ "Ganoderma lucidum"a } & Phenanthrene & 99.65 & \multirow[t]{2}{*}{ Agrawal et al. (2018) } \\
\hline & & Pyrene & 99.58 & \\
\hline & \multirow[t]{3}{*}{ Pycnoporus sanguineus } & Phenanthrene & 90.00 & \multirow[t]{3}{*}{ Munusamy et al. (2008) } \\
\hline & & Pyrene & 96.00 & \\
\hline & & Anthracene & 37.00 & \\
\hline & Armillaria sp. & Pyrene & 63.00 & $\begin{array}{l}\text { Hadibarata and Kristanti } \\
\text { (2013) }\end{array}$ \\
\hline & \multirow[t]{2}{*}{ Pleurotus eryngii } & Anthracene & 99.90 & \multirow[t]{8}{*}{ Li et al. (2009) } \\
\hline & & Benzo[a]pyrene & 87.50 & \\
\hline & \multirow[t]{2}{*}{ Agaricus bisporus } & Anthracene & 89.80 & \\
\hline & & Benzo[a]pyrene & 48.60 & \\
\hline & \multirow[t]{2}{*}{ Pleurotus ostreatus } & Anthracene & 38.00 & \\
\hline & & Benzo[a]pyrene & 31.00 & \\
\hline & \multirow[t]{2}{*}{ Coprinus comatus } & Anthracene & 9.80 & \\
\hline & & Benzo[a]pyrene & 9.30 & \\
\hline \multirow[t]{6}{*}{ Lignin peroxidase } & \multirow[t]{2}{*}{ "Ganoderma lucidum" } & Phenanthrene & 99.65 & \multirow[t]{2}{*}{ Agrawal et al. (2018) } \\
\hline & & Pyrene & 99.58 & \\
\hline & \multirow{3}{*}{$\begin{array}{l}\text { Phanerochaete } \\
\text { chrysosporium }\end{array}$} & Phenanthrene & 72.77 & \multirow[t]{3}{*}{ Wang et al. (2009) } \\
\hline & & Pyrene & 51.16 & \\
\hline & & Benzo[a]pyrene & 25.50 & \\
\hline & Marasmiellus sp. & Pyrene & 100.00 & Vieira et al. (2018) \\
\hline \multirow{5}{*}{$\begin{array}{l}\text { Manganese } \\
\text { peroxidase }\end{array}$} & \multirow[t]{2}{*}{ "Ganoderma lucidum" } & Phenanthrene & 99.65 & \multirow[t]{2}{*}{ Agrawal et al. (2018) } \\
\hline & & Pyrene & 99.58 & \\
\hline & \multirow{3}{*}{$\begin{array}{l}\text { Phanerochaete } \\
\text { chrysosporium }\end{array}$} & Phenanthrene & 72.77 & \multirow[t]{3}{*}{ Wang et al. (2009) } \\
\hline & & Pyrene & 51.16 & \\
\hline & & Benzo[a]pyrene & 25.50 & \\
\hline
\end{tabular}

"The taxonomy of "Ganoderma lucidum" given in the paper by Agrawal et al. (2018) is doubtful since this species has never been safely recorded from India

remediation and other biogeochemical reactions ( $\mathrm{Yu}$ et al. 2016), leading to an increase in microbial growth while reducing Fe(III) minerals in the soil (Kappler et al. 2014).

The effects of biochar on soil fertility have been demonstrated through the $\mathrm{pH}$ increase of the acid found in soils (Van Zwieten et al. 2010), and the benefits in high nutrient levels and retention through the ions absorption (Liang et al. 2006). However, biochar has also been shown to both stabilize and change soil biological community composition and abundance, depending on the biomass source and the temperature used in the pyrolysis reaction (Kim et al. 2012; Lorenz and Lai 2014). Such changes may affect soil structure and nutrient cycles (Gaskin et al. 2010; Rillig and Mummey 2006; Steiner et al. 2008), thereby indirectly affecting plant growth (Warnock et al. 2007). Rhizosphere bacteria and fungi may also directly promote the growth of plants (Compant et al. 2010). Soil fungi (e.g. saprotrophs, pathogens and mycorrhizae) respond differently to biochar applications. Saprotrophic fungi have the potential to modify biochar in the soil through the colonization of the pores present in the structure, and this can lead to decomposition (Atkinson et al. 2010; LipczynskaKochany 2018). Certain arbuscular mycorrhizae are able to increase their root colonization sites in the presence of biochar, enhancing the availability of phosphate to the plant, and thereby negating the need to add artificial fertilizers.

Fungal extracellular enzymes are the agents of both colonization and decomposition, and are becoming increasingly common tools for examining soil microbial response in climate change experiments (Weedon et al. 2011). Saprotrophic fungi are considered to be efficient 
degraders of lignocellulosic biomass due to the wide spectrum of extracellular enzymes they produce. Their production and activities are strongly affected by temperature, moisture and $\mathrm{pH}$. Many studies have been performed on the influence of temperature and moisture on the microbial ecosystem of soils and the concomitant effect on global enzyme pool composition and size (Schimel et al. 2007; Sowerby et al. 2005). Enzyme pool size is controlled by the rate at which enzymes are produced by microbes relative to the rate at which they are degraded by the environment. The production of these extracellular enzymes incurs a cost to the microorganism in terms of energy and nutrients, and so microbes produce certain enzymes targeting specific compounds rich in carbon, nitrogen or phosphorous. Moisture levels influence the diffusion of substrates, the hydration state of the individual enzymes, and the rate of water reactivity.

Due to the chemical nature of biochar, it may be possible to use fungi directly attached to the biosourced material or to use fungi in solid-state fermentation conditions to pretreat the biochar prior to soil addition (a type of composting). Figure 48 provides a scanning electron microscopy (SEM) image of field-aged biochar buried in agricultural soil in which hyphal fragments of fungi were fixed on the biochar surface. It is also possible, through a more biotechnologically sophisticated approach, to harness the power of these secreted enzymes to modify the physicochemical properties of the exposed hydroxyl and carbonyl groups on the surface of the biochar. This will make them more functionally active as a soil amendment for the stimulation of the existing microbial communities.
The structure of biochar is similar to lignin, and so the redox-responsive enzymes, such as laccases and hemeperoxidases produced by Basidiomycetes, Ascomycetes and soft-rot fungi such as Chaetomium globosum, Phialophora malorum, and P. mutabilis are designed to modify these structures, and to generate hydroxyl radicals (Gao et al. 2018). These fungi also control the availability of metal ions in the system, either through their incorporation into the protein structure as co-factors, or chelated in organic acids. While this extracellular process is designed to aid the fungus in the lignin degradation process, these enzymes and chelators could also be a way to control the release or captivation of metals in soils. A modified biochar, with increased surface-active groups, would enhance the soil even further, most notably the activity of the natural soil microbiome, including mycorrhizal fungi. This in turn would further improve the water dynamics, nutrient cycling, and suppression of crop diseases, thereby enhancing the productivity of the soil and the crops that are cultivated in it, while at the same time contributing to carbon sequestration and reduced air contamination in rural areas of the world.

\section{Commodities}

Fungi have been exploited both industrially and commercially in many ways especially when valuable commodities are involved. These organisms offer unique advantages in biotechnology as they can be easily cultured, reproduce quickly and have short life cycles. In this section we
Fig. 48 The scanning electron micrographs of field-aged biochar buried in agricultural soil, showing the outer surface of biochar soil interface (Quilliam et al. 2013; with permission), with the arrow indicating an example of pore blockage (a); spatial heterogeneity and sparsity of internal microbial colonisation (b); internal colonisation by hyphal and single-celled microbes (arrows) (c, d)
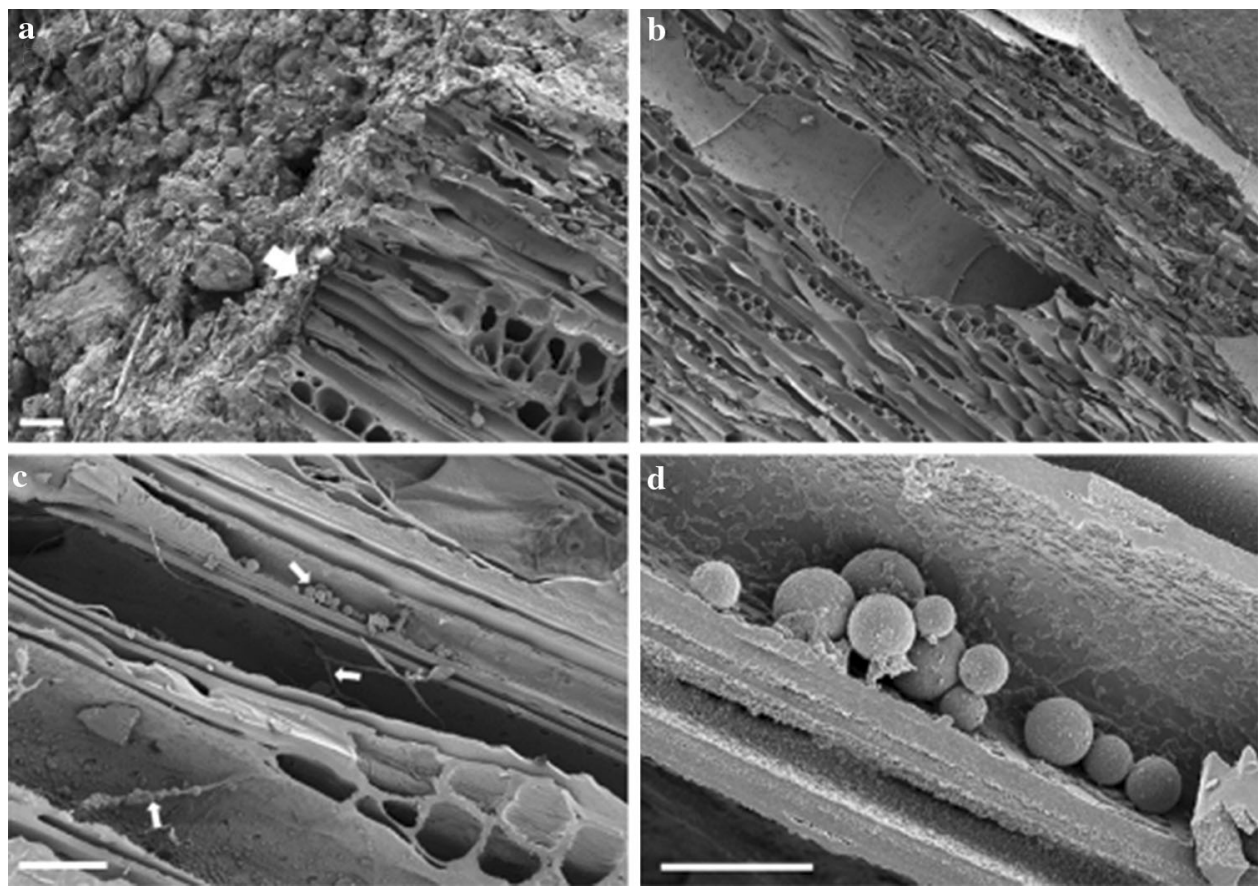
discuss some of the many non-food commodities derived from fungi.

\section{Fungi and cosmetics}

Fungi are used as ingredients in numerous cosmetic products, including in some of the very expensive brands. The beneficial claims are all-encompassing, but many have yet to be proven. As the uses of fungi in cosmetics was reviewed by Hyde et al. (2010), which includinges, skin whiteners, moisturizers, anti-aging, shampoos and many others were reviewed by Hyde et al. (2010), we briefly summarize the topic here. The demand for cosmetic products has rapidly increased, and; hence cosmetics has have become a worldwide industry (Hyde et al. 2010). Cosmetics are mainly classified as cosmeceuticals, applied externally to the skin, such as creams, lotions or ointments, and nutricosmetics, which are consumed as dietary supplements (Hyde et al. 2010). Apart from makeup, cosmeceuticals are categorized as anti-aging, anti-wrinklinge, skin revitalizingation, skin whitening, anti-oxidant and moisturizing products. To avoid any carcinogenic effects, there has been a recent trend towards natural cosmetics, such as fungi-based products (Hyde et al. 2010; Imhoff et al. 2011; Mohd-Nasir and Mohd-Setapar 2018). Furthermore, the potential of fungi to be utilized as "bio factories" to produce nanoparticles in the cosmetic industry is currently being explored (El Enshasy et al. 2018).

Ascomycota and Basidiomycota are extensively used in the cosmetic industry (Hyde et al. 2010). Secondary metabolites extracted from the mycelia or fruiting bodies and ingredients from fungal fermentation are used as cosmetic ingredients. Aspergillus species (Hyde et al. 2010) and Rhizopus species (Mucoromycota) are used in the production of lactic acid, which is a main ingredient in both anti-aging and skin whitening cosmetics. Lactic acid is mainly used to hydrate and make the skin smooth. Additionally, in peeling lotions, lactic acid is contained in higher concentrations and helps to remove the outer layer of the skin (Zhang et al. 2007). Fungi are utilized in the production of anti-oxidants, fatty acids and polysaccharides in anti-aging products, such as the chitin-glucan complexes of Aspergillus niger and some mushroom species (Synytsya et al. 2009; Vysotskaya et al. 2009).

Eicosapentaenoic acid is a rare omega fatty acid used in anti-aging products. It is extracted from Mortierella species (Wang et al. 2007). Mortierella and Rhizopus species produce $\gamma$-linolenic acid. This compound is used as an antiinflammatory agent and facilitates healthy skin (Krištofíková et al. 1991). Sporotrichum pruinosum is used to produce melanocytic enzymes through a submerged aerobic fermentation process. Melanocytic enzyme is used in some skin whitening cosmetics to activate the

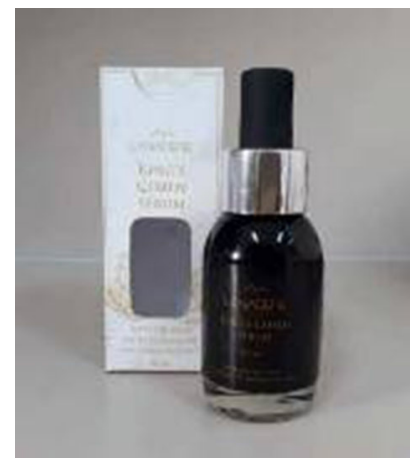

Fig. 49 The King's Cordy Serum contains Cordyceps militaris extract. The extract is claimed to facilitate anti-wrinkle effects and add moisture and antioxidants to the skin. It is produced in the Centre of Excellence in Fungal Research in Mae Fah Lung University, Chiang Rai, Thailand

depigmentation of the skin (Mohorčič et al. 2007). To treat neurodermatitis and sclerodermatitis, an extract obtained from Tremella sp. is used. Extracts of Ophiocordyceps sinensis and Tremella fuciformis are utilized to increase the moisturising effect in certain cosmetic products (Hyde et al. 2010) (Fig. 49).

\section{Agarwood}

Agarwood is an economically valuable resinous heartwood product derived from wounded trees of the family Thymalaeaceae (Novriyanti et al. 2010; Subasinghe et al. 2012; Peng et al. 2015a; Chowdhury et al. 2016; Chen et al. 2018b). Agarwood incenses are used for fragrance in soaps and shampoos and have a pleasant aroma and general perfume and are an element of important religious rituals in Ayurvedic, Tibetan and traditional East Asian medicine (Subasinghe et al. 2012; Rhind 2013; Chowdhury et al. 2016; Lee and Mohamed 2016; López-Sampson and Page 2018) and as aromatic food ingredients (Liu et al. 2013; Tan et al. 2019). India as well as the Southeast Asian countries are the main manufactures of agarwood products, while China, India, the Middle East and Japan are the primary consumer countries. Species of Aquilaria (Adams et al. 2014; Mohamed et al. 2014; Selvan et al. 2014: Azren et al. 2019), Gyrinops, Aetoxylon and Gonystylus are used for the production of agarwood (Subasinghe et al. 2012; Mohamed et al. 2014; Mohamed and Rasool 2016). Currently, agarwood producing Aquilaria species are cultivated from the home garden level to large scale plantations in Southeast Asia, India and southern China (Lee and Mohamed 2016; Azren et al. 2019).

Naturally, agarwood formation occurs through wounds. The infected tissues produce oleoresin which is converted to odoriferous aromatic agarwood resin (Peng et al. 2015a; Chowdhury et al. 2016). When microbial pathogens enter 
Fig. 50 Barks of Gyrinops walla Gaertn. (Thymelaeaceae) in Sbaragmuwa University premises, Sri Lanka 2018 a Healthy bark, b, c damaged bark (Photo credit: H.A.T. Chinthaka)

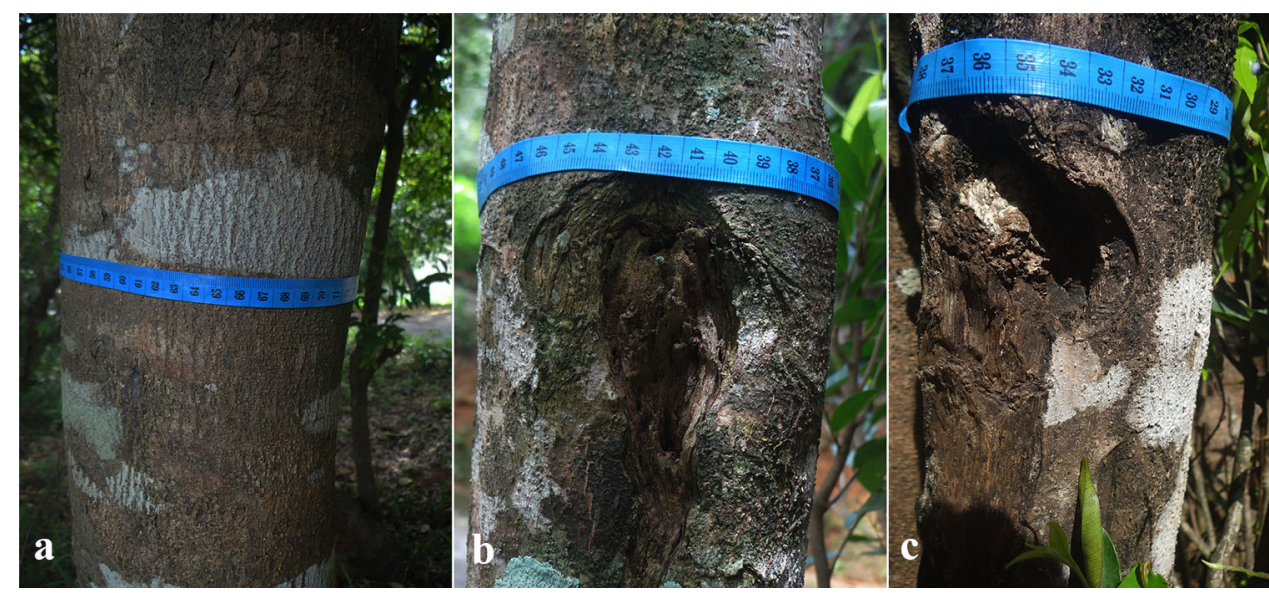

the wound, the defense mechanism of the trees is triggered (Mohamed et al. 2014; Chowdhury et al. 2016; Azren et al. 2019). Sesquiterpenes and 2-(2-phenylethyl) chromone derivatives are the key active compounds in agarwood (Chen et al. 2011; Naef 2011; Mohamed et al. 2014; Li et al. 2019; Tan et al. 2019).

Agarwood causal agents are divided into chemical, physical, and biological agents. Fungi are the biological agents (Novriyanti et al. 2010; Chhipa et al. 2017). Depending on the stress, the tree forms either physical or chemical defense mechanisms (Mohamed and Rasool 2016; Chhipa et al. 2017). The defense substances produced act as biochemical or biological defense agents (Mohamed and Rasool 2016). The wounded or infected tree stem turns dark brownish or black (Fig. 50, Adams et al. 2014). After infection agarwood resin is secreted by the tree and deposited around the wound for number of years. Resinous agarwood form perfumed compounds and is a rare natural mechanism which is poorly understood (Sen et al. 2015). Volatile compounds eventually result in agarwood (Tan et al. 2019). Other than natural agarwood formation, artificial agarwood inducing methods have been developed. Biological inocula such as microbes and fungi are key agents for non-conventional artificial agarwood formation (Azren et al. 2019).

Research has been carried out to determine which fungi are responsible for agarwood production and some of the isolated taxa are listed in Table 23. However, the role of individual fungi needs extensive research to establish which species are important in the process.

\section{Fungal enzymes}

Enzymes are biocatalysts that are involved in catalysis reactions without needing extreme conditions, such as very high temperatures, high pressures or corrosive environments, all of which are often required in chemical processes. Enzymes often offer a competitive advantage when compared to chemical catalysts. The enzymatic approach is environmentally friendly, as it requires mild conditions and does not normally result in the production of toxic byproducts (Chapla et al. 2012). Enzymes are used to catalyze reactions in production processes of several sectors including industrial bioconversion (biocatalyst), environmental bioremediation, agricultural sectors and also biotransformations of numerous compounds such as flavonoids (Das and Rosazza 2006; Wohlgemuth 2010; Choi et al. 2015). There are several sources of enzymes including animals, plants and microorganisms (bacteria, fungi and protists). Microbial enzymes have generally been used because of their easier isolation in high amounts, lowcost production, stability at various extreme conditions, and their co-compounds, which are also more controllable and less harmful. Microbial enzymes secreted into the media are highly reliable for industrial processes and applications. Microbes isolated from different sources even among species and strains of the same genus may produce varying levels of enzymes of differing properties.

Fungal enzymes have attracted attention for several applications because fungi can grow on low cost materials and secrete large amounts of enzymes into the culture medium, which eases downstream processing (Anitha and Palanivelu 2013). Several fungal enzymes are available commercially including amylases, cellulases, lipases, phytases, proteases, and xylanases (Saxena et al. 2005; Srilakshmi et al. 2015). The positive environmental impact of the production processes is of general interest and the use of enzymatic reactions instead of organic solvents or chemical reactions is highly valued. Figure 51 and Table 24 show examples of important fungal enzymes and the enzyme sources that are used in many applications, but only a few fungal strains meet the criteria for commercial production.

Most applications of enzymes in the food industry have focused on hydrolytic reactions (Akoh et al. 2008; Choi et al. 2015). Glycoside hydrolases and $\beta$-galactosidase are 
Table 23 Fungal species potentially responsible for the formation of agarwood in Aquilaria species

\begin{tabular}{|c|c|c|}
\hline Host species & Agarwood responsible fungi & References \\
\hline Aquilaria sp. & $\begin{array}{l}\text { Chaetomium globosum } \\
\text { Fusarium oxysporum } \\
\text { F. moniliforme } \\
\text { F. sambucinum } \\
\text { F. solani } \\
\text { F. tricinctum }\end{array}$ & $\begin{array}{l}\text { Budi et al. (2010) } \\
\text { Azren et al. (2019) } \\
\text { Tamuli et al. (2000) }\end{array}$ \\
\hline A. agallocha & $\begin{array}{l}\text { Aspergillus tamarii } \\
\text { Fusarium solani } \\
\text { Botryodiplodia theobromae } \\
\text { Phialophora parasitica } \\
\text { Cytosphaera mangiferae }\end{array}$ & $\begin{array}{l}\text { Bhattacharyya et al. (1952) } \\
\text { Gibson (1977) } \\
\text { Jalaluddin (1977) } \\
\text { Azren et al. (2019) }\end{array}$ \\
\hline A. crassna & $\begin{array}{l}\text { Acremonium sp. } \\
\text { Fusarium sp. }\end{array}$ & Azren et al. (2019) \\
\hline A. malaccensis & $\begin{array}{l}\text { Alternaria } \mathrm{sp} . \\
\text { Cladosporium } \mathrm{sp} . \\
\text { Curvularia } \mathrm{sp} . \\
\text { Fusarium proliferatum } \\
\text { Phaeoacremonium } \mathrm{sp} . \\
\text { Trichoderma } \mathrm{sp} .\end{array}$ & $\begin{array}{l}\text { Ma et al. (2012) } \\
\text { Premalatha and Kalra (2013) }\end{array}$ \\
\hline A. sinensis & $\begin{array}{l}\text { A. niger } \\
\text { Botryosphaeria dothidea } \\
\text { Fusarium solani } \\
\text { Lasiodiplodia theobromae } \\
\text { Melanotus flavolivens } \\
\text { Paraconiothyrium variabile }\end{array}$ & $\begin{array}{l}\text { Lin et al. (2010) } \\
\text { Cui et al. (2013) } \\
\text { Tian et al. (2013) } \\
\text { Zhang et al. (2014a, b) } \\
\text { Mohamed et al. (2014) } \\
\text { Chen et al. (2017) } \\
\text { Azren et al. (2019) }\end{array}$ \\
\hline A. subintegra (agarwood oil) & $\begin{array}{l}\text { Arthrinium sp. } \\
\text { Colletotrichum sp. }\end{array}$ & $\begin{array}{l}\text { Monggoot et al. (2017) } \\
\text { Azren et al. (2019) }\end{array}$ \\
\hline
\end{tabular}

involved in the production of prebiotics, a dietary substance composed of non-starch polysaccharides and oligosaccharides, such as inulin fructo-oligosaccharides, galacto-oligosaccharides, lactulose, and breast milk oligosaccharides, that selectively promote the growth of beneficial intestinal microorganisms in humans (Torres et al. 2010). Cellulases, pectinases, and xylanases are widely used for the clarification of juices and wines. Amylases hydrolyze starch in the starch liquefaction process and convert starch into glucose syrups (Souza and Magalhaes 2010). Tannases are used to reduce tannin levels in food products such as fruit juices, tea, beer, and wines (Yao et al. 2014). These enzymes are also used to hydrolyze gallic acid esters and produce gallic acid, which is used as a substrate for the synthesis of food preservatives (Belmares et al. 2004; Srivastava and Kar 2009; Yao et al. 2014; Dhiman et al. 2017).

Starch is used as the strengthening agent in the desizing process to prevent the breaking of the warp thread used within the weaving process in the textile industry. Amylases are used for desizing starch in the textile industry because they yield products that do not degrade the fibers. The use of $\alpha$-amylases in the pulp and paper industry is for the modification of starch of coated paper and textiles. The quality of textiles and paper coated with desizing starch is protected against mechanical damage during processing and finishing. The recycling of waste paper is an ecofriendly trend in the paper industry. The enzymatic reaction of cellulases, lipases, pectinases and xylanases aid in the removal of contaminated ink (Bhat 2000). Proteases and cellulases are used in the polishing step for clear dyeing to improve color and surface vividness, and resist textile wrinkling. Lignin peroxidases, manganese peroxidases, laccases and xylanases are all used to enhance the quality of the pulp by removing lignin and hemicelluloses, which are typical impurities in the pulp and paper industries (Choi et al. 2015). Protease is used in leather processing for the dehairing and debating of skins and hides instead of 
Fig. 51 Fungal enzymes and applications

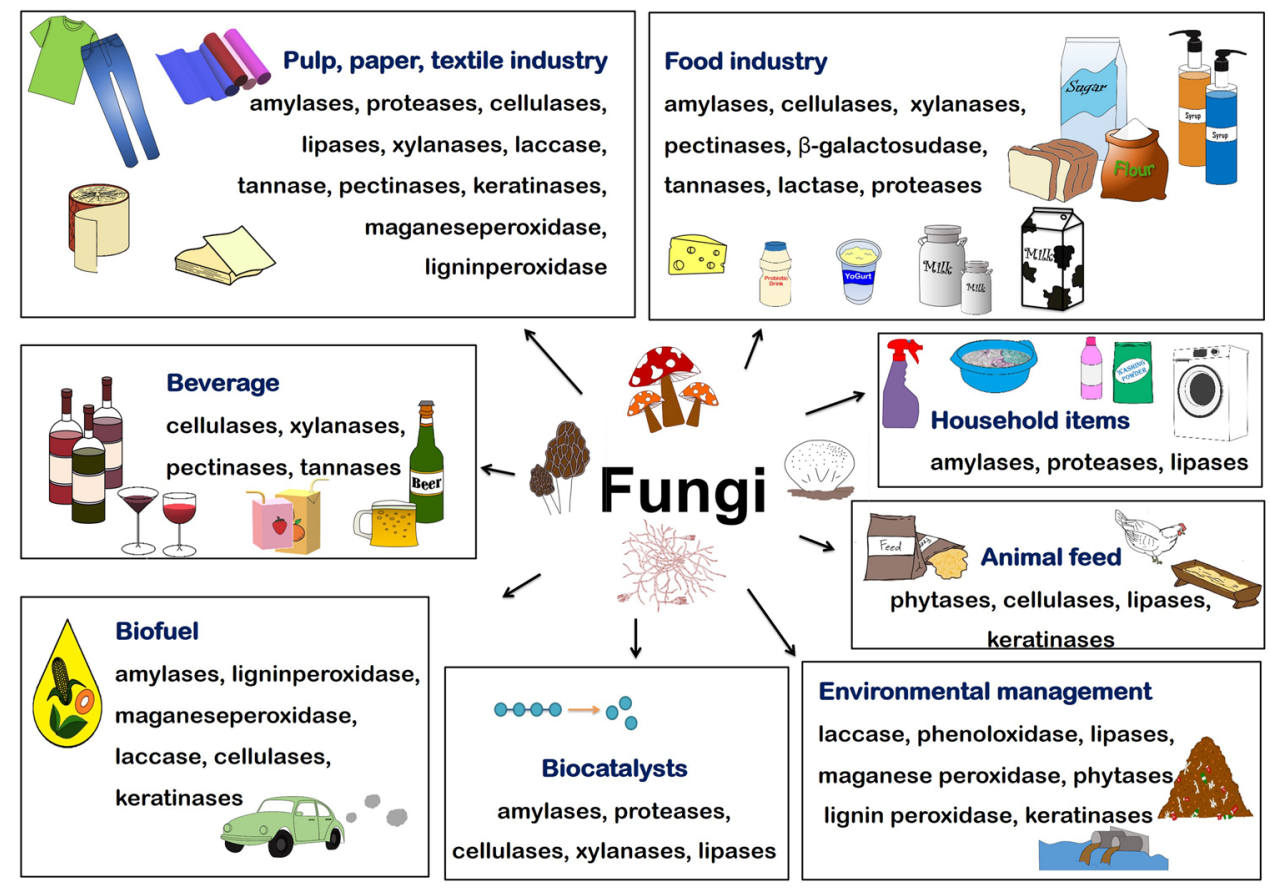

chemical processes that have negative implications for the environment. Tannases are used for high-grade leather tannin preparation in the leather industry (Dhiman et al. 2017). This enzymatic process is eco-friendly and helps to manage waste resulting from production processes.

Proteases, lipases and amylases are used for dishwashing detergents (Souza et al. 2015). Amylases are the second type of enzymes used in the formulation of enzymatic detergent, and $90 \%$ of all liquid detergents contain amylases (Souza and Magalhaes 2010).

Cellulases, xylanases and $\beta$-glucanases have been used in cereal-based feed for monogastric animals such as pigs and poultry, which are unable to fully degrade and utilize plant-based feeds containing high amounts of cellulose and hemicellulose (Bhat 2000; Kirk et al. 2002). Adding phytases to the feed improves utilization of natural phosphorus bound in phytic acid in cereal-based feed for monogastrics. Normally, $85-90 \%$ of the total plant phosphorus is bound to phytic acid, which makes it difficult to monogastrics to utilize. Therefore, available inorganic phosphates have to be supplemented to the feed in order to reach the required concentration. Phytases can minimize the need for supplementation of inorganic phosphorus in the feed (Kirk et al. 2002).

Basidiomycetes can enzymatically attack the polymers in the complex-structured lignocellulose. The appearance of white rot fungi involves the nutrient recycling of wood required for new plant growth with evolutionary impact on plant diversification (Kües 2015). Dioxygenases, laccases, peroxidases, and phenoloxidases are enzymes used in wastewater treatment (Durán and Esposito 2000). Fungi are recognized as one of the best lipase sources among microorganisms (Facchini et al. 2015). The most important step to improving the biological degradation of fatty wastewater is pretreatment procedures in order to hydrolyze and dissolve fats, which can accelerate the process by decreasing the fat adsorption to the surface of the anaerobic sludge while not limiting the transport of the soluble substrate to the biomass (Valladao et al. 2009; Facchini et al. 2015). Effluents from slaughterhouses contain high concentrations of biodegradable organic matter, most of which consists of lipids and proteins with low degradability. Consequently, before effluents are released into the environment, it is necessity to reduce fat oil and protein from these wastewaters. A potential application of enzyme treatments is a reduction of the organic matter, which contributes to a cleaner effluent (Valladao et al. 2011). Moreover, white rot fungi produce lignin degrading enzymes, such as manganese peroxidases, lignin peroxidases and laccases that have been used in biotechnology for degradation of broad-spectrum refractory organic pollutants and bioremediation of polycyclic aromatic hydrocarbons (PAHs) and chlorinated hydrocarbons in the environment (Gao et al. 2010).

Biotechnological applications for practical use have limiting factors, as they require large amounts of enzymes. Hence, the production of low-cost and readily available enzymes possessing satisfactory operating characteristics is 
Table 24 Examples of enzyme sources and applications of fungi

\begin{tabular}{|c|c|c|c|}
\hline Enzymes & Applications & Fungal sources & References \\
\hline Amylases & $\begin{array}{l}\text { Hydrolysis of starch in starch processing industry; food and dairy } \\
\text { industry; textile industry; pulp and paper industry; detergent } \\
\text { industry; pharmaceutical industry; animal feed industry }\end{array}$ & $\begin{array}{l}\text { Aspergillus fumigatus } \\
\text { Aspergillus niger } \\
\text { Cylindrocephalum } \mathrm{sp} . \\
\text { Lentinula edodes } \\
\text { Penicillium citrinum } \\
\text { Penicillium fellutanum } \\
\text { Rhizopus stolonifer }\end{array}$ & $\begin{array}{l}\text { Goto et al. (1998) } \\
\text { Ko et al. (2005) } \\
\text { Kathiresan and } \\
\text { Manivannan (2006) } \\
\text { Sunitha et al. (2012) } \\
\text { Sahoo et al. (2014) } \\
\text { Saleem and Ebrahim } \\
\text { (2014) }\end{array}$ \\
\hline Cellulases & $\begin{array}{l}\text { Animal feed industry; pulp and paper industry; detergent industry; } \\
\text { food processing; juices and wines clarification; textile industry; } \\
\text { biomass conversion into biofuels }\end{array}$ & $\begin{array}{l}\text { Aspergillus niger } \\
\text { Lentinula edodes } \\
\text { Trichoderma } \\
\text { longibrachiatum } \\
\text { Volvariella diplasia }\end{array}$ & $\begin{array}{l}\text { Ko et al. (2005) } \\
\text { Pachauri et al. (2017) } \\
\text { Puntambekar (1995) }\end{array}$ \\
\hline Keratinases & $\begin{array}{l}\text { Biomass conversion into biofuels; hydrolysis of keratinous wastes } \\
\text { such as feather, hair, and horn; eliminating horny epithelial } \\
\text { cells adhering to textile fibers; reducing the environmental pollution }\end{array}$ & $\begin{array}{l}\text { Aspergillus oryzae } \\
\text { Aspergillus parasiticus } \\
\text { Doratomyces microspores } \\
\text { Paecilomyces marquandii }\end{array}$ & $\begin{array}{l}\text { Anitha and Palanivelu } \\
\text { (2013) } \\
\text { Farag and Hassan (2004) } \\
\text { Friedrich et al. (2005) } \\
\text { Gradisar et al. (2000) } \\
\text { Kim (2007) } \\
\text { Santos et al. (1996) } \\
\text { Veselá and Friedrich } \\
\text { (2009) }\end{array}$ \\
\hline Laccase & $\begin{array}{l}\text { Biopulping biobleaching deinking in pulp and paper industry; lignin } \\
\text { degradation; pharmaceutical industry; removal of phenolic } \\
\text { substances and stabilize the beverage; biomass conversion into } \\
\text { biofuels }\end{array}$ & $\begin{array}{l}\text { Agaricus subrufescens (as } \\
\text { "blazei") } \\
\text { Coniophora puteana } \\
\text { Ganoderma sp. } \\
\text { Omphalotus olearius } \\
\text { Phanerochaete floridensis } \\
\text { Pleurotus ostreatus }\end{array}$ & $\begin{array}{l}\text { Arora et al. (2002) } \\
\text { Ergun and Urek (2017) } \\
\text { Lee et al. (2004) } \\
\text { Ko et al. (2005) } \\
\text { Songulashvili et al. (2007) } \\
\text { Ullrich et al. (2005) }\end{array}$ \\
\hline $\begin{array}{l}\text { Lignin } \\
\text { peroxidase }\end{array}$ & Lignin degradation; biomass conversion into biofuels & $\begin{array}{l}\text { Aspergillus sclerotiorum } \\
\text { Cladosporium } \\
\text { cladosporioides } \\
\text { Mucor racemosus } \\
\text { Phanerochaete } \\
\text { chrysosporium } \\
\text { Sparassis latifolia }\end{array}$ & $\begin{array}{l}\text { Bonugli-Santos et al. } \\
\text { (2010) } \\
\text { Chandrasekaran et al. } \\
\text { (2014) } \\
\text { Johjima et al. (1999) } \\
\text { Wen et al. (2009) }\end{array}$ \\
\hline Lipases & $\begin{array}{l}\text { Degradation of fat in wastewater treatment; animal feed industry; pulp } \\
\text { and paper industry; detergent industry; food processing; leather } \\
\text { processing; textile industry; pharmaceutical industry }\end{array}$ & $\begin{array}{l}\text { Aspergillus sp. } \\
\text { Curvularia } \text { sp. } \\
\text { Fusarium solani } \\
\text { Fusarium verticillioides } \\
\text { Penicillium } \text { sp. } \\
\text { Penicillium restrictum } \\
\text { Penicillium wortmanii } \\
\text { Rhizopus oligosporus } \\
\text { Trichoderma } \text { sp. } \\
\text { Trichoderma atroviride } \\
\text { Trichoderma harzianum } \\
\text { Mucor } \text { sp. }\end{array}$ & $\begin{array}{l}\text { Costa and Peralta (1999) } \\
\text { El-Ghonemy et al. (2017) } \\
\text { Facchini et al. (2015) } \\
\text { Maia et al. (2001) } \\
\text { Marques et al. (2014) } \\
\text { Nwuche and Ogbonna } \\
\text { (2011) } \\
\text { Ul-Haq et al. (2002) } \\
\text { Ülker et al. (2011) } \\
\text { Valladao et al. (2011) }\end{array}$ \\
\hline
\end{tabular}


Table 24 continued

\begin{tabular}{|c|c|c|c|}
\hline Enzymes & Applications & Fungal sources & References \\
\hline $\begin{array}{l}\text { Manganese } \\
\text { peroxidase }\end{array}$ & Lignin degradation; biomass conversion into biofuels & $\begin{array}{l}\text { Lentinula edodes } \\
\text { Phlebia radiata } \\
\text { Omphalotus olearius } \\
\text { Phellinus robustus }\end{array}$ & $\begin{array}{l}\text { Arora et al. (2002) } \\
\text { Grabski et al. (1998) } \\
\text { Songulashvili et al. (2007) }\end{array}$ \\
\hline Pectinases & Juices and wines clarification; textile industry & $\begin{array}{l}\text { Aspergillus japonicus } \\
\text { Aspergillus oryzae } \\
\text { Penicillium viridicatum } \\
\text { Penicillium chrysogenum } \\
\text { Thermoascus aurantiacus }\end{array}$ & $\begin{array}{l}\text { Banu et al. (2010) } \\
\text { Biz et al. (2016) } \\
\text { Martins et al. (2002) } \\
\text { Semenova et al. (2003) } \\
\text { Silva et al. (2002) }\end{array}$ \\
\hline Proteases & $\begin{array}{l}\text { Detergent industry; food processing; pharmaceutical industry; leather } \\
\text { processing; textile industry }\end{array}$ & $\begin{array}{l}\text { Aspergillus } \text { sp. } \\
\text { Fomitella fraxinea } \\
\text { Humicola } \text { sp. } \\
\text { Mucor } \text { sp. } \\
\text { Penicillium sp. } \\
\text { Pleurotus citrinopileatus } \\
\text { Rhizopus sp. } \\
\text { Thermoascus } \mathrm{sp} . \\
\text { Thermomyces } \mathrm{sp} .\end{array}$ & $\begin{array}{l}\text { Cui et al. (2007) } \\
\text { Lee et al. (2006) } \\
\text { Souza et al. (2015) }\end{array}$ \\
\hline Phytases & $\begin{array}{l}\text { Feed supplement in diets in animal feed industry; improvement of soil } \\
\text { fertilization and nutrient uptake by plants; reduction the excretion of } \\
\text { phosphorus in manure; reducing phosphate pollution in soil and } \\
\text { water }\end{array}$ & $\begin{array}{l}\text { Aspergillus sp. } \\
\text { Aspergillus niger } \\
\text { Mucor sp. } \\
\text { Rhizopus oligosporus } \\
\text { Rhizomucor pusillus }\end{array}$ & $\begin{array}{l}\text { Bei et al. (2009) } \\
\text { Brugger et al. (2004) } \\
\text { Casey and Walsh (2004) } \\
\text { Chadha et al. (2004) } \\
\text { Saxena et al. (2005) }\end{array}$ \\
\hline Tannases & $\begin{array}{l}\text { Food processing; juices and wines clarification and removal of } \\
\text { phenolic substances and stabilize the beverage; reduction of } \\
\text { hydrolysable tannin in poultry feeds; production of gallic acid from } \\
\text { gallotannins }\end{array}$ & $\begin{array}{l}\text { Aspergillus aculeatus } \\
\text { A. awamori } \\
\text { A. caespitosus } \\
\text { A. niger } \\
\text { A. versicolor } \\
\text { Penicillium charlesii } \\
\text { P. crustosum } \\
\text { P. variable } \\
\text { P. restrictum }\end{array}$ & $\begin{array}{l}\text { Ahmed and Rahman } \\
\text { (2014) } \\
\text { Bagga et al. (2015) } \\
\text { Batra and Saxena (2005) } \\
\text { Srivastava and Kar (2009) } \\
\text { Mahapatra et al. (2005) }\end{array}$ \\
\hline Xylanases & $\begin{array}{l}\text { Pulp and paper industry; animal feed industry; bread-making; juice } \\
\text { and wine industries; xylitol production; prebiotics production; food } \\
\text { processing; textile industry; juices and wines clarification; biomass } \\
\text { conversion into biofuels }\end{array}$ & $\begin{array}{l}\text { Aspergillus foetidus } \\
\text { A. niger } \\
\text { Talaromyces amestolkiae }\end{array}$ & $\begin{array}{l}\text { Chapla et al. (2012) } \\
\text { de Alencar Guimaraes } \\
\text { et al. (2013) } \\
\text { Nieto-Domínguez et al. } \\
\text { (2017) }\end{array}$ \\
\hline
\end{tabular}

challenging. After all, each industrial application may require specific properties of the biocatalysts. Moreover, researchers still face challenges in finding new enzymes that could result in novel applications through better isolation, study of enzyme stability at extreme conditions and purification procedures. The selection of enzymes for thermostable and stringent conditions is important for industrial processes and applications. Therefore, the search for new microorganisms that contain the desired properties is a continuous process. Furthermore, the production and expression of recombinant enzymes through protein engineering technology should also be investigated in order to obtain enzymes of desired characteristics in a specific host cell. 
Industrial enzymes are normally produced in bioreactors that contain up to over 200,000 litres and are subsequently purified in the industrial downstream processes. Therefore, the fungi that can be used for such processes must be fast growing but not be pathogens or mycotoxin producers. The use of heterologous expression, recombinant DNA technology and gene cloning to improve the production yield of enzymes, as well as their activity, can circumvent the difficulties associated with the production of large quantities.

\section{Preservatives}

Aside from the antibiotics that are used in human and veterinary medicine, secondary metabolites of fungi that exert antimicrobial activities may also have great use in the food and cosmetics industries, e.g. as food or cosmetics ingredients. In these markets, natural compounds are interesting if they (1) show significant biological activities in relevant test systems; (2) are derived from edible species; (3) are devoid of significant toxicity and (4) can be made available at low costs of goods.

Attempts have been made to replace classical synthetic preservatives, such as benzoic and sorbic acids, by natural ingredients, predominantly by plant-derived extracts and compounds (Davidson et al. 2013; Ribes et al. 2017). In fact, fungal metabolites are ideal candidates for such natural preservatives, since they can be produced in large quantities by biotechnological processes.
A recent success story relating to the successful development of such a class of molecules is based on the glycolipids from the fan-shaped jelly fungus, Dacryopinax spathularia and other species of the basidiomycete class Dacrymycetales. These compounds are characterized by rather broad spectrum antimicrobial effects, which have precluded their development as classical antibiotics, because such compounds should at least have selective activities against either pathogenic fungi (eukaryotes) or bacteria (prokaryotes), but not both. Ideal candidates for antibiotics should also address a defined, specific molecular target that is only present in the pathogens (examples see entry on antibacterials, e.g. enzymes catalysing glucan synthesis for antimycotics or peptidoglucan synthesis for antibacterial antibiotics). In the classical search for antibiotics such non-selective compounds that have broadspectrum would have been sorted out at an early stage because of lacking selectivity. However, as described in a patent application (Stadler et al. 2012), the glycolipids from D. spathularia (Fig. 52) had particularly strong activities against acidophilic yeasts and food and beverage spoilage bacteria such as Lactobacillus plantarum, when the serial dilution assays were carried out in "natural" matrices such as apple juice. The fungus Dacryopinax spathularia is regarded as edible (Ao et al. 2016; Boa 2004) in various countries of the world and its edible basidiomata contain glycolipids (M. Stadler et al. unpubl.). Moreover, the activity persisted for up to several weeks and even yeast strains such as Zygosaccharomyces bailii that

Fig. 52 Chemical structure of one of the major glycolipids from Dacryopinax spathularia and an images of its basidiomes in the natural habitat<smiles>CCCCC(CCCC(O)C(O)CCCCCCCCCCCCCCC(O)C(=O)O)OC1OCC(O)C(O)C1OC1COC(OC(C)=O)C(O)C1OC1OC(COC(=O)CC(C)C)C(O)C(O)C1O</smiles>
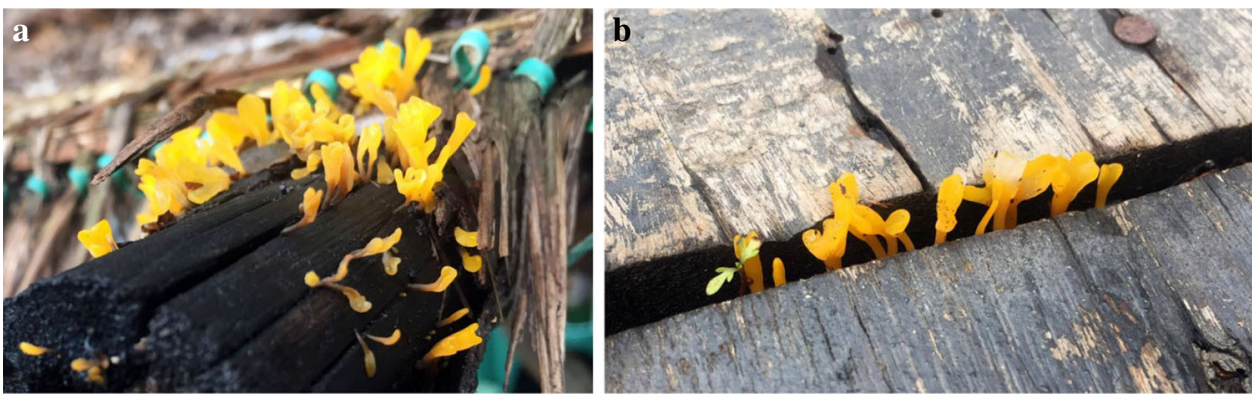
had become sensitive to the commonly used non-natural preservatives, such as benzoic acid and sorbic acid (cf. Kuanyshev et al. 2017), were found to be highly sensitive. These data have only been reported in the patent application by Stadler et al. (2012) and were not yet published in a peer-reviewed scientific journal. The production process for the glycolipids was further developed to attain them in multi-gram and even $\mathrm{kg}$ scale, and at the same time, the downstream processing procedure was simplified substantially, leading to a drastic decrease of the cost of goods. The material was then subjected to in vivo studies, demonstrating favorable pharmacokinetics (Bitzer et al. 2017a) as well a lack of toxicity (Bitzer et al. 2017b, c, 2018). Recently, the large German chemical company Lanxess has licensed the project and the glycolipids are now being produced at industrial scale as natural preservative agents. Curiously, the glycolipids of Dacryopinax species can be produced by a wide variety of genera in the Dacrymycetales, and the patent application by Stadler et al. (2012) even included a claim based on homology of $28 \mathrm{~S}$ rDNA sequences to define the potential producer organisms. The production of these compounds seems to be a valid chemotaxonomic feature that has been "preserved" in the producer organisms in the course of their evolution, probably as the fungi have a selective advantage over their competitors.

A recent review by Günther et al. (2017) has also outlined the potential of glycolipids, which can be produced at industrial scale by yeast-like Basidiomycota such as Ustilago and Pseudozyma species (Ustilagomycetes), for other industrial applications. These compounds are biosurfactants and can be used as natural emulsifiers for various applications. They have potential to replace the currently used petrochemical products in cosmetics, as well as pharmaceutical or biomedical applications and may even be useful for bioremediation technologies, such as solubilisation and removal of oils from contaminated soil. However, for many of these applications, the costs of goods are presently still prohibitive, and the existing biotechnological production processes remain to be optimized further.

\section{Organic acids}

Organic acids are low molecular weight compounds which contain functional groups with acidic properties such as carboxyl, sulfonic, thiol and alcohol groups (Yin et al. 2015). Organic acids have a vast array of applications in different fields including food and beverages, pharmaceuticals, cosmetics and agriculture. Before the advancement of industrial biotechnology, most of the building block chemicals such as organic acids involved in industrial scale chemical production were derived from petroleum sources
(Sauer et al. 2008). Nowadays, many organic acids are being produced at industrial scale using different fungal species, relying on sustainable biotechnological processes that can easily compete with organic synthesis (Table 25). Natural metabolic pathways or genetically modified natural product biosynthetic pathways of these fungi are favored in low cost production of organic chemicals with certain functional groups that require costly oxidative processes in industrial scale chemical production (Sauer et al. 2008). Fungi produce a diverse range of organic acids in vitro as well as in the natural environment and many species can tolerate very low $\mathrm{pH}$ values. These capabilities may constitute a competitive advantage, since they can "acidify" the environment to inhibit the growth of other microorganisms and to solubilize soil metals for easy absorbance. In this entry, we focus mainly on organic acids containing carboxyl functional groups with industrial scale applications.

\section{Applications and production:}

\section{Citric acid}

Citric acid is a tri-carboxylic acid which is extensively used in the food and beverage industry as an acidulate, $\mathrm{pH}$ adjuster, flavoring agent, emulsifier in ice-cream (He et al. 2019) and processed cheese production, and antimicrobial agent. Furthermore, citric acid is employed in food as an acidulant and antioxidant additive, in therapeutic apheresis as an anticoagulant (Lee and Arepally 2012), in cosmetics and toiletries as a buffering agent with metal ion chelating abilities, and in industrial scale applications such as metal cleaning for metal oxide removal from metal surfaces (Soccol et al. 2006).

Before the discovery of industrial scale citric acid biosynthesis with Aspergillus niger using submerged fermentation techniques (Currie 1917; Show et al. 2015), it was commercially produced in England beginning around 1826 by direct extraction from Italian lemons (Papagianni 2007; Show et al. 2015). Other species of Aspergillus, such as A. awamori, A. fonsecaeus, A. nidulans, A. phoenicis, A. saitoi and $A$. wentii, have also been reported to accumulate citric acid in considerable quantities (Soccol et al. 2006; Papagianni 2007). The organic substrates for citric acid biosynthesis by fermentation of starch hydrolysates include sugarcane broth, cane molasses and beet molasses (Soccol et al. 2006). A recombinant strain of the yeast, Yarrowia lipolytica, has also been successfully utilized in citric acid production using sucrose as the starting material (Fig. 53) (Förster et al. 2007).

\section{Fumaric acid}

Fumaric acid was first isolated from the plant Fumaria officinalis and the acid is a building block component in polymerization and esterification reactions. High purity 
Table 25 Organic acids produced by fungi and diversity of their applications

\begin{tabular}{|c|c|c|c|c|}
\hline Organic acids & Organisms & Applications & Chemical formula & Molecular structure \\
\hline Citric acid & $\begin{array}{l}\text { Aspergillus awamori } \\
\text { Aspergillus flavus } \\
\text { Aspergillus fonsecaeus } \\
\text { Aspergillus nidulans } \\
\text { Aspergillus niger } \\
\text { Aspergillus phoenicis } \\
\text { Aspergillus saitoi } \\
\text { Aspergillus wentii } \\
\text { Yarrowia lipolytica }\end{array}$ & $\begin{array}{l}\text { Cosmetics } \\
\text { Food and beverages } \\
\text { Metal cleaning } \\
\text { Pharmaceuticals } \\
\text { Toiletries }\end{array}$ & $\mathrm{C}_{6} \mathrm{H}_{8} \mathrm{O}_{7}$ & $\mathrm{HO}^{\prime}$ \\
\hline Fumaric acid & $\begin{array}{l}\text { Rhizopus arrhizus } \\
\text { Rhizopus formosa } \\
\text { Rhizopus nigricans } \\
\text { Rhizopus oryzae }\end{array}$ & $\begin{array}{l}\text { Bio-polymers } \\
\text { Cattle feed } \\
\text { Food and beverages } \\
\text { Pharmaceuticals }\end{array}$ & $\mathrm{C}_{4} \mathrm{H}_{4} \mathrm{O}_{4}$ & $\mathrm{O}=$ \\
\hline Gluconic acid & Aspergillus niger & $\begin{array}{l}\text { Derivatization reactions } \\
\text { Food and beverages }\end{array}$ & $\mathrm{C}_{6} \mathrm{H}_{12} \mathrm{O}_{7}$ & (3O \\
\hline Itaconic acid & $\begin{array}{l}\text { Aspergillus terreus } \\
\text { Saccharomyces cerevisiae } \\
\text { Ustilago maydis }\end{array}$ & $\begin{array}{l}\text { Coatings } \\
\text { Detergents } \\
\text { Polymers (poly-MMA) } \\
\text { Rubber industry } \\
\text { Super-absorbents }\end{array}$ & $\mathrm{C}_{5} \mathrm{H}_{6} \mathrm{O}_{4}$ & $\mathrm{HO}^{\prime}$ \\
\hline Lactic acid & $\begin{array}{l}\text { Recombinant yeast strains } \\
\text { Rhizopus oryzae }\end{array}$ & $\begin{array}{l}\text { Biodegradable polymers } \\
\text { Cosmetics } \\
\text { Food industry } \\
\text { Oral hygiene products } \\
\text { Bio-polymers } \\
\text { Derivatization reactions }\end{array}$ & $\mathrm{C}_{3} \mathrm{H}_{6} \mathrm{O}_{3}$ & $\mathrm{O}_{\mathrm{OH}}^{\mathrm{O}}$ \\
\hline Succinic acid & Rhizopus sp. & & $\mathrm{C}_{4} \mathrm{H}_{6} \mathrm{O}_{4}$ & 0 \\
\hline
\end{tabular}

grade fumaric acid is administered in the management of psoriasis in humans (Balak 2015) and has shown to significantly reduce methane emission by cattle, when added as a supplement in cattle feed (Roa Engel et al. 2008). It also has applications in food and beverage industry as an acidulant and flavor enhancing agent. Fumaric acid can 


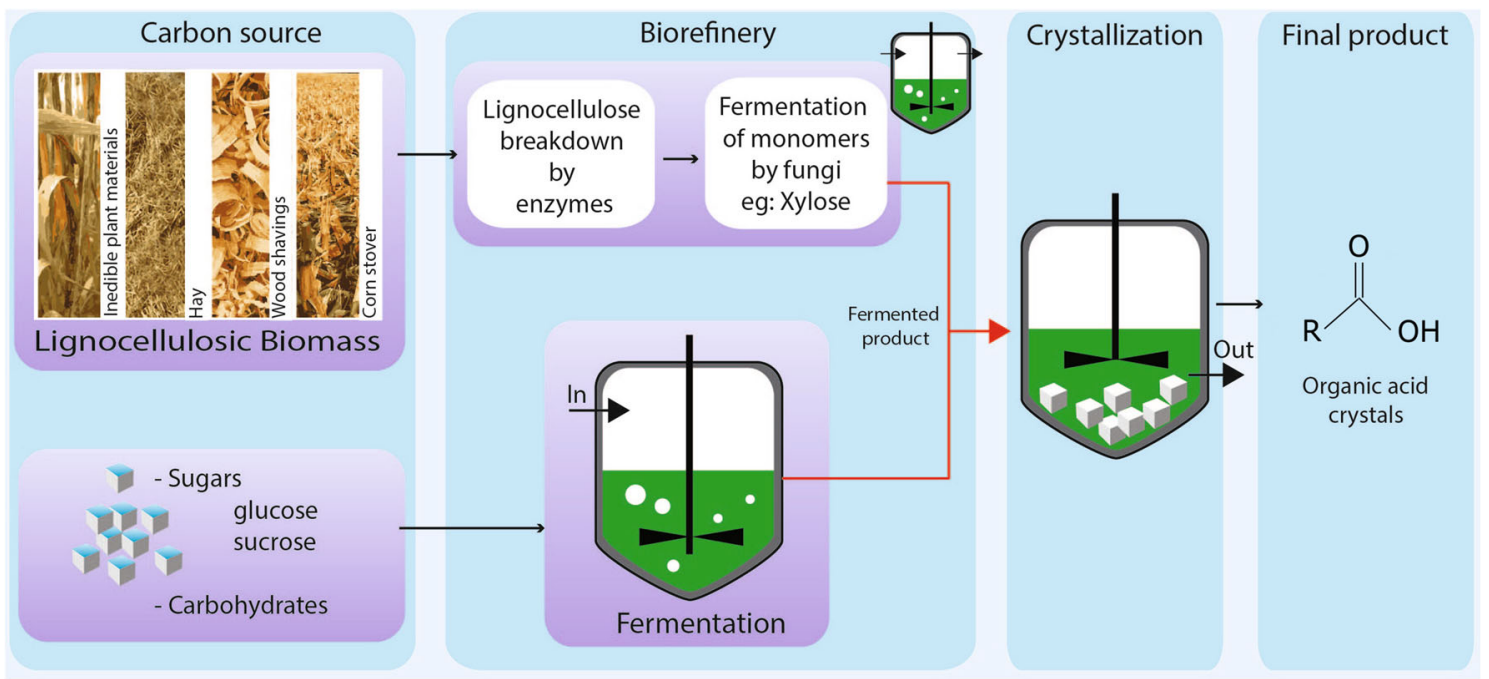

Fig. 53 Schematic representation of general organic acid production procedure using fungi

undergo derivatization reactions to produce malic acid and L-aspartic acid (Goldberg et al. 2006; Roa Engel et al. 2008). Bio-based fumaric acid is produced by the fermentation of different substrates such as glucose by Rhizopus arrhizus, $R$. nigricans, and $R$. oryzae, molasses by $R$. arrhizus and cassava bagasse by $R$. formosa (Table 25) (Roa Engel et al. 2008; Xu et al. 2012).

\section{Gluconic acid}

Since the chemical structure of gluconic acid combines both, carboxylic and alcohol functional groups, it can undergo various derivatization reactions including intra molecular esterification. Gluconic acid has applications in the food and beverage industry as a sensory property enhancer, acidity regulator and preservative for pickled food and processed meat products (Canete-Rodriguez et al. 2016). Gluconic acid is produced by surface or submerged fermentation procedures with various types of input materials such as starch, lignocellulosic biomass, sugarcane molasses, whey, waste paper, figs, banana must and grape must as carbon source using Aspergillus niger strains (Table 25) (Canete-Rodriguez et al. 2016). During the fermentation process, glucose in the medium is converted extracellularly by the enzymatic reaction of glucose oxidase by A. niger (Magnuson and Lasure 2004).

\section{Itaconic acid}

Itaconic acid has applications in detergents, coatings and in the rubber industry. Poly(acrylamide-co-itaconic acid) a super absorbent polymer which is used to absorb aqueous solutions is also derived from itaconic acid (Choi et al. 2015). The most on demand industrial scale application is the conversion of itaconic acid to methyl methacrylate
(MMA) and the production of poly MMA, commonly known as Plexiglass (Becker et al. 2015; Choi et al. 2015). The acid is generally produced via fermentation of glucose using Aspergillus terreus. Furthermore, Ustilago maydis and Saccharomyces cerevisiae are also capable of producing itaconic acid by glucose fermentation (Table 25) (Becker et al. 2015).

\section{Lactic acid}

Lactic acid is widely used in food industry in yoghurt and cheese production, cosmetic industry in skin care products, and oral hygiene products (Martinez et al. 2013). One of the most important uses of lactic acid is the manufacturing of polylactic acid which has applications in the textile industry and food packaging utensils (Sauer et al. 2008; Bozell and Petersen 2010; Martinez et al. 2013).

Lactic acid is mostly produced by fermentation with lactic acid bacteria (Sauer et al. 2008); however, the exploitation of Rhizopus oryzae has shown promising outcomes by producing higher titers of lactic acid of $280 \mathrm{~g} /$ $\mathrm{L}$ as anhydrous calcium lactate with $0.92 \mathrm{~g} / \mathrm{g}$ productivity of glucose input under fed-batch cultures (Yamane and Tanaka 2013). Xylose, a pentose sugar derived from lignocellulosic biomass hydrolysis, can also be utilized as an input carbon source for lactic acid biosynthesis. Xylose fermentation is carried out by employing recombinant yeast strains (Sauer et al. 2010) and $R$. oryzae, grown in mineral medium (Magnuson and Lasure 2004; Maas et al. 2006) (Table 25). The extracellular release of amylases by fungi is an added advantage of the fermentation process in hydrolyzing starch from various input sources, especially by the species of Rhizopus (Martinez et al. 2013). 


\section{Succinic acid}

Succinic acid acts as a building block component for the synthesis of valuable chemicals, such as 1,4-butanediol, tetrahydrofuran, $\gamma$-butyrolactone, N-methylpyrrolidone, and even in the synthesis of bio-based polymers such as polybutylene succinate and polyester polyols, which may eventually replace conventional petrol-derived polymers in the future (Choi et al. 2015). An improved fermentation process for Rhizopus species was patented by DuPont to increase the TCA cycle based carboxylic acid production by limiting the dissolved oxygen levels in fermenters between 30 and $80 \%$. During this fermentation process fumaric acid, succinic acid and L-malic acid are produced (Ling and $\mathrm{Ng}$ 1989). In a recent study $209.7 \mathrm{~g} / \mathrm{L}$ succinic acid titre was obtained under fed batch fermentation conditions using Yarrowia lipolytica fed with crude glycerol as the carbon source (Li et al. 2018).

Commercial scale production of organic acids is highly dependent on strain performance. Since most of the wild type strains do not produce commercially acceptable yields, fermentation conditions need to be modified according to the requirement and strain (Xu et al. 2012). To meet industrial scale requirements, the wild type strains are being genetically modified or subjected to classical mutagenesis with the goal to increase production. Recently, it was reported that the deletion of the mitochondrial fumarase gene and introduction of succinate/fumarate transporter increased fumaric acid production in Saccharomyces cerevisiae (Yin et al. 2015). A high yielding fumaric acid producing Rhizopus oryzae RUR709 strain was developed by UV and $\gamma$-ray mutagenesis and finally produced $32.1 \mathrm{~g} / \mathrm{L}$ fumaric acid-a concentration 1.9-fold higher than the wild type strain (Huang et al. 2010). Glucose oxidase deficient mutants of Aspergillus carbonarius have shown increased citric acid production by reducing by-product (gluconic acid) formation through an increase of the carbon flux towards the reductive tricarboxylic acid pathway (Yang et al. 2014b).

These metabolic engineering techniques have led to the industrial scale production of natural products by modifying natural biosynthetic pathways to increase productivity or synthesize novel derivatives to meet the global demand for organic acids.

\section{Textile dyes}

Fungi are important as natural dye producers in textile industries (Mapari et al. 2010; Chadni et al. 2017). The textile industry is the largest consumer of organic pigments and synthetic dyes (Mapari et al. 2010). Tons of dyes are lost as effluents and can result in environmental pollution and serious human health problems, especially if their release is not properly treated. Colour fastness to washing and crocking are essential qualities for any dye/ fabric combination. Fungal species that are used in textile dyeing are listed in Table 26.

Hernández et al. (2018a) reported that wood rotting fungi can be used in textile dyeing. Suciatmih (2002) studied the effect of colouring $\mathrm{pH}$ and mordant on fungal dyes quality using woolen yarn (Fig. 54). In their study, three isolates of Aspergillus, three isolates of Penicillium, two isolates of Paecilomyces, one isolate of Monascus purpureus and one isolate of Trichoderma harzianum were used with two different mordants (alum and $\mathrm{FeSO}_{4}$ ) by three different dying $\mathrm{pH}(3,6$, and 9). The results indicated that low $\mathrm{pH}$ value exhibited a strong colour as well as the mordant $\mathrm{FeSO}_{4}$ produced almost darker colour.

When used in manufactured products, concerns related to the presence of toxic metabolites in pigments and dyes produced by fungi are commonly raised. In this regard, the correct identification of fungal strains and the implementation of toxicity tests are key factors to ensure a safe environment for workers and harmless products for end users (Hernández et al. 2018a). Regarding the broad spectrum of the fungal pigments, fungi including edible and non-edible mushrooms are a resource of natural dyes.

\section{The future}

\section{Functional genomics and the search for novel anti-invectives}

Functional genomics is the approach used to study functions and interactions of genes and their products in a specific context (Liu et al. 2010a; Buza and McCarthy 2013). This requires sequencing of the entire genome. Sequencing techniques have come a long way, since the release of the first whole fungal genome of Saccharomyces cerevisiae (Goffeau et al. 1996). Today the combining of newly arising techniques like PacBio with Illumina to provide fully closed genomes has led to numerous fungal genome databases, such as the fungal genome initiative (Haas et al. 2011), comprising over 100 fungal genomes, or FungiDB (Stajich et al. 2012) and Ensembl Fungi (Kersey et al. 2010) with over 1000 fungal genomes. Out of many examples that target various product categories, we will here only give some examples on what is feasible now for the secondary metabolite biosynthesis due to the employment of-OMICS and bioinformatics tools and synthetic biotechnology.

The genomes of the Fungi contain many genes and gene clusters that encode for potentially beneficial products, including industrially important enzymes as well as secondary metabolites. Also the bioinformatics tools for the 
Table 26 Examples of pigments produced by fungi

\begin{tabular}{|c|c|c|c|}
\hline Fungal producer & Pigment name/colour & Application & References \\
\hline Acrostalagmus sp. & Brown and reddish brown & & Atalla et al. (2011) \\
\hline Agaricus xanthodermus & Yellow & & Hanson (2008) \\
\hline \multirow[t]{2}{*}{ Alternaria alternata } & & Dyeing of wool and silk & Sharma et al. (2012) \\
\hline & Brown and reddish brown & & Atalla et al. (2011) \\
\hline Amanita muscaria & Betains(Orange-red & & Li and Oberlies (2005) \\
\hline Aspergillus niger & Brown and reddish brown & & Atalla et al. (2011) \\
\hline Aspergillus sp. & Yellow & Dyeing of cotton, silk and silk cotton & $\begin{array}{l}\text { Anchana devi (2014), Kumar et al. } \\
\text { (2017), Sarkar et al. (2017) }\end{array}$ \\
\hline $\begin{array}{l}\text { Boletales (Gomphidiaceae } \\
\text { and Suillaceae) }\end{array}$ & Yellow pulvinic acid derivatives & & Knight and Pattenden (1976) \\
\hline Bisporomyces sp. & Brown and reddish brown & & Atalla et al. (2011) \\
\hline Chlorociboria aeruginosa & Xylindein/Green & $\begin{array}{l}\text { Colourfast dyeing of cotton, } \\
\text { polyamide, polyester and wool }\end{array}$ & $\begin{array}{l}\text { Weber et al. (2014), Hinsch and } \\
\text { Robinson (2016) }\end{array}$ \\
\hline $\begin{array}{l}\text { Cortinarius cinnabarinus } \\
\text { (European toadstool) }\end{array}$ & Fallacinol/ Dark orange & & Gill (1994) \\
\hline Cortinarius violaceus & Striking deep violet colour & & Von Nussbaum et al. (1998) \\
\hline Cortinarius sp. & $\begin{array}{l}\text { Dimeric anthraquinone derivative/ } \\
\text { Bright yellow }\end{array}$ & & Velišek and Cejpek (2011) \\
\hline Cunninghamella sp. & Brown and reddish brown & & Atalla et al. (2011) \\
\hline Curvularia lunata & & Dyeing of wool and silk & Sharma et al. (2012) \\
\hline Dermocybe sanguinea & $\begin{array}{l}\text { Anthraquinone compounds as } \\
\text { aglycones }\end{array}$ & & $\begin{array}{l}\text { Hynninen et al. (2000), Räisänen } \\
\text { et al. (2000) }\end{array}$ \\
\hline Dermocybe sp. & $\begin{array}{l}\text { Dimeric anthraquinone derivative/ } \\
\text { Bright yellow }\end{array}$ & & Velišek and Cejpek (2011) \\
\hline Fusarium oxysporum & Anthraquinone/Purple & Dyeing of wool & $\begin{array}{l}\text { Nagia and El-Mohamedy (2007), } \\
\text { Hernández et al. (2018a) }\end{array}$ \\
\hline Gymnopilus sp. & $\begin{array}{l}\text { Yellow-brown styrylpyrone } \\
\text { pigments bis-noryangonin and } \\
\text { hispidin }\end{array}$ & & Gill (1994) \\
\hline $\begin{array}{l}\text { Hymenochaetaceae } \\
\text { (Inonotus, Onnia and } \\
\text { Phellinus) }\end{array}$ & Yellow pigment hispolon & & Lee and Yun (2006) \\
\hline Hypholoma sp. & $\begin{array}{l}\text { Yellow-brown styrylpyrone } \\
\text { pigments bis-noryangonin and } \\
\text { hispidin }\end{array}$ & & Gill (1994) \\
\hline Hygrocybe sp. (witchs' hat) & Muscaflavin/yellow & & Li and Oberlies (2005) \\
\hline Penicillium chrysogenum & Brown and reddish brown & & Atalla et al. (2011) \\
\hline Penicillium italicum & Brown and reddish brown & & Atalla et al. (2011) \\
\hline Penicillium minioluteum & & Dyeing of web blue goat nappa skin & Gupta and Aggarwal (2016) \\
\hline Penicillium murcianum & Yellow & Dyeing of wool & Hernández et al. (2018a, b) \\
\hline Penicillium oxalicum & Brown and reddish brown & & Atalla et al. (2011) \\
\hline Penicillium regulosum & Brown and reddish brown & & Atalla et al. (2011) \\
\hline Penicillium sp. & & Dyeing of cotton and silk & Sarkar et al. (2017) \\
\hline Pholiota sp. & $\begin{array}{l}\text { Yellow-brown styrylpyrone } \\
\text { pigments bis-noryangonin and } \\
\text { hispidin }\end{array}$ & & Gill (1994) \\
\hline Phymatotrichum sp. & Brown and reddish brown & & Atalla et al. (2011) tested \\
\hline Scytalidium cuboideum & Draconin red & $\begin{array}{l}\text { Colourfast dyeing of cotton, } \\
\text { polyamide, polyester and wool } \\
\text { Colourfasten }\end{array}$ & $\begin{array}{l}\text { Weber et al. (2014), Hinsch and } \\
\text { Robinson (2016) }\end{array}$ \\
\hline $\begin{array}{l}\text { Scytalidium } \\
\text { ganodermophthorum }\end{array}$ & Yellow & $\begin{array}{l}\text { Colourfast dyeing of cotton, } \\
\text { polyamide, polyester and wool }\end{array}$ & $\begin{array}{l}\text { Weber et al. (2014), Hinsch and } \\
\text { Robinson (2016) }\end{array}$ \\
\hline
\end{tabular}


Table 26 continued

\begin{tabular}{|c|c|c|c|}
\hline Fungal producer & Pigment name/colour & Application & References \\
\hline Suillus grevillei & $\begin{array}{l}\text { Contains at least } 11 \text { yellow, orange } \\
\text { and red pigments }\end{array}$ & & Besl and Bresinsky (1997) \\
\hline Talaromyces australis & Red & Dyeing of wool & Hernández et al. (2018a, b) \\
\hline Talaromyces sp. & Orange & & $\begin{array}{l}\text { Morales-Oyervides et al. (2017), } \\
\text { Hernández et al. (2018a) }\end{array}$ \\
\hline Talaromyces verruculosus & Red & $\begin{array}{l}\text { Adequate colour tone for cotton } \\
\text { fabric without any cytotoxic effect }\end{array}$ & Chadni et al. (2017) \\
\hline Thermomyces sp. & Yellow & Dyeing of silk & (Poorniammal et al. 2013) \\
\hline Trichoderma spirale & Yellow & & Hernández et al. (2018a) \\
\hline Trichoderma virens & & $\begin{array}{l}\text { Dyeing of wool and silk and have } \\
\text { antifungal properties }\end{array}$ & Sharma et al. (2012) \\
\hline Trichoderma sp. & & Dyeing of cotton, silk and silk cotton & Anchana devi (2014) \\
\hline Tricholoma sp. & $\begin{array}{l}\text { Dimeric anthraquinone derivative/ } \\
\text { Bright yellow }\end{array}$ & & Velišek and Cejpek (2011) \\
\hline Xylaria polymorpha & Blackish brown & & Kumar et al. (2017) \\
\hline
\end{tabular}

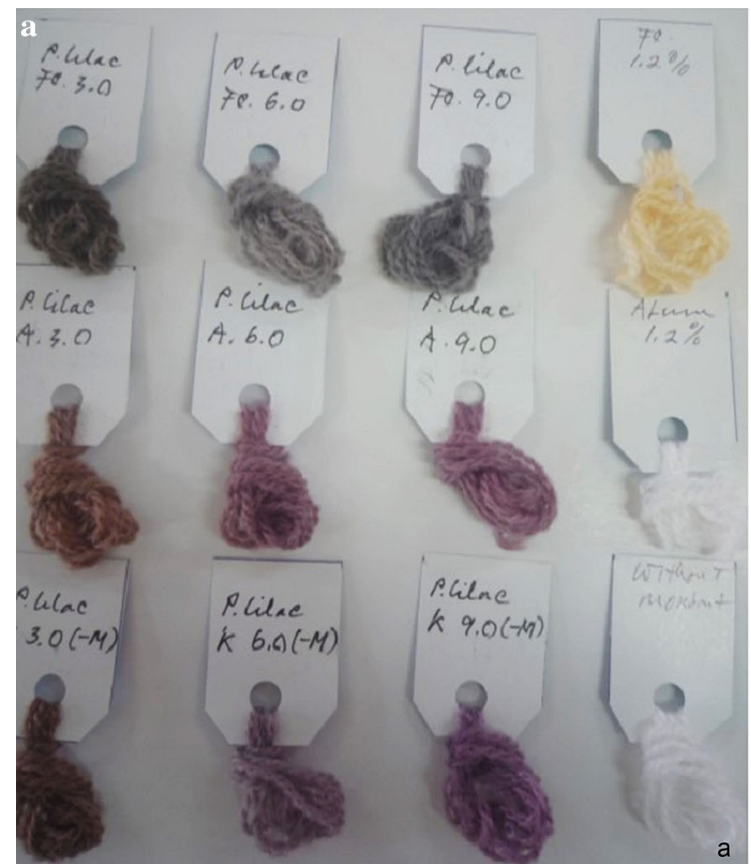

Fig. 54 a Colour variation on woolen yarn dyed with Purpureocillium lilacinum dye with different mordants and dyeing $\mathrm{pH}$. b Colour variation on woolen yarn dyed with Penicillium sp. dye with different

annotation of genomes have significantly improved and today, next to the long established antiSMASH (Blin et al. 2017), fungi-specific algorithms for identifying biosynthetic gene clusters, like the FunGeneClusterS (Vesth et al. 2016) exist. These tools have enabled researchers to heterologously express such gene clusters in well-known hosts, such as Aspergillus niger (Boecker et al. 2018). This strategy can be used not only to facilitate an easier production of a known metabolite at industrial scale, but also

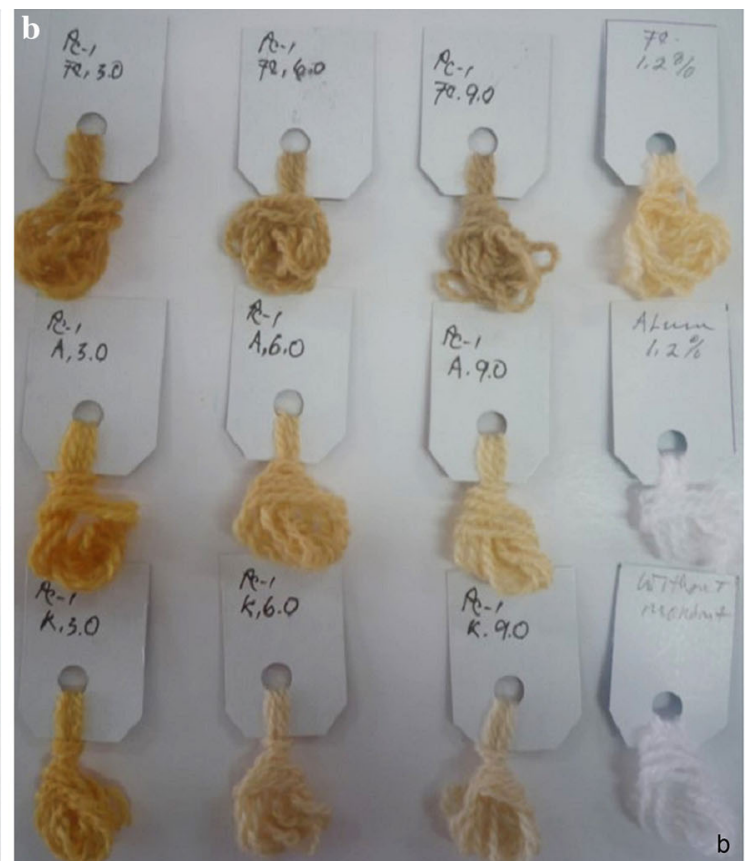

mordants and dyeing $\mathrm{pH}\left(\mathrm{Fe}=\mathrm{FeSO}_{4} \cdot 7 \mathrm{H}_{2} \mathrm{O}, \mathrm{A}=\right.$ alum and $\mathrm{K}=\mathrm{pH}$ control) (courtesy of Suciatmih)

to express previously silent gene clusters, which were found while analysing the genome - a technique often referred to as 'genome mining', offering a whole new source for the discovery of novel anti-infectives. Heterologous expression of specific gene clusters in a different host now also allows for the elucidation of biosyntheses of known antibiotics/natural products. One of the first fungal biosynthesis studied in such manner, was this of the mycotoxin trichothecene (Tokai et al. 2007). Over the 

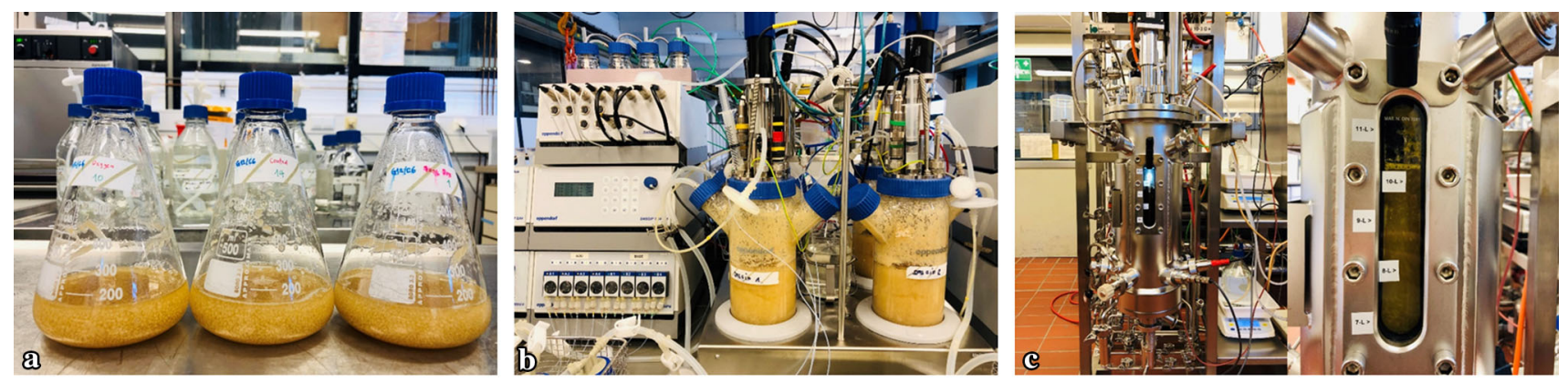
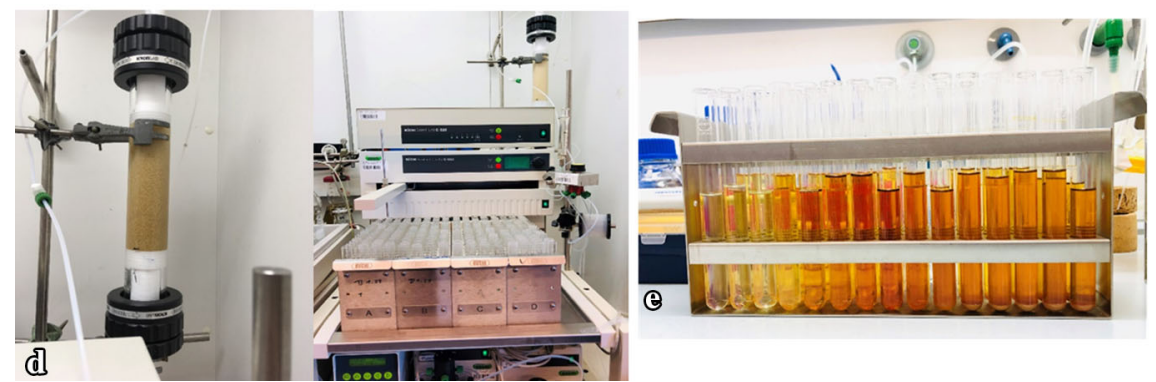

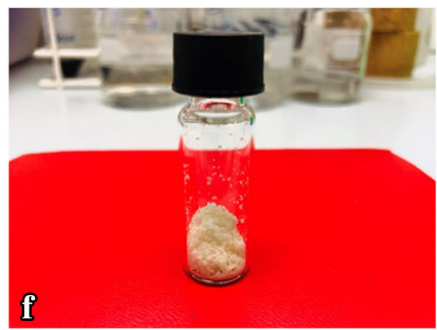

Fig. 55 Stages of the production and isolaton of a biologically active metabolite from the cultures of a basidiomycete (Omphalotus nidiformis). a Submerged cultivation in shake flasks. b Stirred tank fermentation in a parallel 1.5 L Bioreactor systems. C. Bench-Scale $10 \mathrm{~L}$ stirred tank. D. Column chromatography for fractionation and

years, many other studies on Ascomycota have followed, but as of recently even the Basidiomycota have been targeted for elucidation of the biosynthesis of their secondary metabolites (Lin et al. 2019). Most recently, the biosynthesis of the antifungal strobilurins (see entry on antimycotics and fungicides), has been elucidated, through expression in Aspergillus oryzae (Nofiani et al. 2018). In the future, modifying the biosynthesis of a fungal metabolite in order to enhance its production at commercial scale or to achieve adjusted, better bioavailable drugs, may well become the norm. Studies on the regulation of secondary metabolite biosynthesis (Brakhage and Schroeckh 2010) have also been developed in model organisms and are now available for broad applications across the fungal kingdom. This may soon lead to the discovery of totally novel classes of metabolites, using genome mining, which was already demonstrated for enzymes (e.g. Dilokpimol et al. 2018). Figure 55 illustrates the production and isolation procedure for a bioactive metabolite from a basidimycete culture, which was obtained in very high yields in a relatively short time, owing to the fact that modern bioprocess technology and methods of systems biology were employed.

Acknowledgements This work was supported by the Strategic Priority Research Program of the Chinese Academy of Sciences, Grant No. XDB31000000. Naritsada Thongklang would like to thank Thailand research fund grants "Study of saprobic Agaricales in Thailand to find new industrial mushroom products" (Grant No. DBG6180015) and Mae Fah Luang University grant "Optimal compound recovery using the polymeric adsorbent XAD. E. Eluted fractions from compound purification. F. Crystals of pure compound. Images by Teresa Briem and Lillibeth Chaverra-Muñoz, HZI, Braunschweig, Germany

conditions for domestication and biological activities of selected species of Ganoderrma" (Grant No. 621C1535). K.D. Hyde and Naritsada Thongklang would like to thanks to Thailand research fund grants "Domestication and bioactive evaluation of Thai Hymenopellis, Oudemansiella, Xerula and Volvariella species (basidiomycetes)" (Grant No. DBG6180033). K.D. Hyde thanks the financial support from the Visiting Professor grant at Chiang Mai University, Thailand and KIB. The authors acknowledge the contribution of M.M. Vasanthakumari, K.M. Manasa and P. Rajani, in various stages of preparation of the manuscript. Samantha C. Karunarathna thanks CAS President's International Fellowship Initiative (PIFI) for funding his postdoctoral research (Number 2018PC0006), and the National Science Foundation. Associate Professor R Jeewon thanks University of Mauritius for support. Binu C. Samarakoon offers her sincere gratitude to the "National Research Council of Thailand" (NRCT Grant No. 256108A3070006) for the financial support. Peter E Mortimer would like to thank the National Science Foundation of China and the Chinese Academy of Sciences for financial support under the following Grants: 41761144055, 41771063, Y4ZK111B01. M. Doilom would like to thank Chiang Mai University, the 5th batch of Postdoctoral Orientation Training Personnel in Yunnan Province and the 64th batch of China Postdoctoral Science Foundation. T.S. Suryanarayanan thanks the United States-India Educational Foundation (USIEF), New Delhi and the Fulbright Scholar Program (USA) for the award of a Fulbright-Nehru Senior Researcher grant to conduct research in the Department of Chemistry and Biochemistry, The Ohio State University, USA. Thanks to Research and Researchers for Industries Grant (PHD57I0015) for financial support to Boontiya Chuankid. Birthe Sandargo is grateful to the Deutsche Forschungsgemeinschaft (DFG) for a PhD grant. Clara Chepkirui is indebted to a $\mathrm{PhD}$ stipend from the German Academic Exchange Service (DAAD) and the Kenya National Council for Science and Technology (NACOSTI). Kevin D Hyde would also like to thank the National Research Council of Thailand grants Thailands' Fungal Diversity, Solving Problems and Creating Biotechnological Products (Grant No. 61201321016). This work is partly supported by the Department of 
Biotechnology, Government of India, New Delhi (Chemical Ecology of the North East Region (NER) of India: A collaborative programme Linking NER and Bangalore Researchers; DBT-NER/Agri/24/2013) and Indian Council of Agricultural Research (ICAR-CAAST-Project F.No./NAHEP/CAAST/2018-19), Government of India, New Delhi.

Open Access This article is distributed under the terms of the Creative Commons Attribution 4.0 International License (http://crea tivecommons.org/licenses/by/4.0/), which permits unrestricted use, distribution, and reproduction in any medium, provided you give appropriate credit to the original author(s) and the source, provide a link to the Creative Commons license, and indicate if changes were made.

\section{References}

Abdel-Fattah GM, Ibrahim AH, Al-Amri SM, Shoker AE (2013) Synergistic effect of arbuscular mycorrhizal fungi and spermine on amelioration of salinity stress of wheat ("“Triticum aestivum L." cv. 9). Aust J Crop Sci 7:1525

Abdelaziz ME, Kim D, Ali S, Fedoroff NV, Al-Babili S (2017) The endophytic fungus Piriformospora indica enhances Arabidopsis thaliana growth and modulates $\mathrm{Na}^{+} / \mathrm{K}^{+}$homeostasis under salt stress conditions. Plant Sci 263:107-115

Abe M, Takaoka N, Idemoto Y, Takagi C et al (2008) Characteristic fungi observed in the fermentation process for Puer tea. Int $\mathrm{J}$ Food Microbiol 124:199-203

Abraham WR, Estrela AB (2016) Fungal metabolites for the control of biofilm infections. Agriculture 6:37

Adams SJ, Manohara T, Krishnamurthy K, Kumar TS (2014) Histochemical studies on fungal-induced agarwood. Indian $\mathrm{J}$ Plant Sci 5:102-110

Adekunle AE, Guo C, Liu CZ (2016) Lignin-enhanced laccase production from Trametes versicolor. Waste Biomass Valori 8:1061-1066

Adrio JL, Demain AL (2003) Fungal biotechnology. Int Microbiol 6:191-199

Aggangan NS, Pollisco MA, Bruzon JB, Gilbero JS (2013) Growth of Shorea contorta Vid. inoculated with eucalyptus ectomycorrhiza fungi in the nursery and in a logged-over dipterocarp forest in Surigao, Philippines. Am J Plant Sci 4:896-904

Agrawal N, Verma P, Shahi SK (2018) Degradation of polycyclic aromatic hydrocarbons (phenanthrene and pyrene) by the ligninolytic fungi Ganoderma lucidum isolated from the hardwood stump. Bioresour Bioprocess 5:11

Ahmed ME, Rahman A (2014) Detection of the perfect condition to produce the tannase from Aspergillus niger at different medium. J Babylon Univ Pure Appl Sci 22:1363-1371

Akatin MY (2013) Characterization of a $\beta$-glucosidase from an edible mushroom, Lycoperdon pyriforme. Int J Food Prop 16:15651577

Akihisa T, Uchiyama E, Kikuchi T, Tokuda H et al (2009) Antitumor-promoting effects of 25-methoxyporicoic acid A and other triterpene acids from Poria cocos. J Nat Prod 72:1786-1792

Akiyama K, Matsuzaki K, Hayashi H (2005) Plant sesquiterpenes induce hyphal branching in arbuscular mycorrhizal fungi. Nature 435:824-827

Akoh CC, Chang SW, Lee GC, Shaw JF (2008) Biocatalysis for the production of industrial products and functional foods from rice. J Agric Food Chem 56:10445-10451

Al-Hawash AB, Alkooranee JT, Abbood HA, Zhang J et al (2018) Isolation and characterization of two crude oil-degrading fungi strains from Rumaila oil field, Iraq. Biotechnol Rep 17:104-109
Al-Hazmi AS, Schmitt DP, Sasser JN (1982) The effect of Arthrobotrys conoides on Meloidogyne incognita population densities in corn as influenced by temperature, fungus inoculum density, and time of fungus introduction in the soil. J Nematol $14: 168$

Alananbeh KM, Bouqellah NA, Al Kaff NS (2014) Cultivation of oyster mushroom Pleurotus ostreatus on date-palm leaves mixed with other agro-wastes in Saudi Arabia. Saudi J Biol Sci 21:616625

Alapi EM, Fischer J (2006) Table of selected analogue classes. In: Fischer J, Ganellin CR (eds) Analogue-based drug discovery. Wiley-VCH, Weinheim, pp 441-552

Albermann S, Elter T, Teubner A, Krischke W, Hirth T, Tudzynski B (2013) Characterization of novel mutants with an altered gibberellin spectrum in comparison to different wild-type strains of Fusarium fujikuroi. Appl Microbiol Biotechnol 97(17):77797790

Alberts AW, Chen J, Kuron G, Hunt V et al (1980) Mevinolin: a highly potent competitive inhibitor of hydroxymethylglutarylcoenzyme A reductase and a cholesterol-lowering agent. Proc Natl Acad Sci USA 77:3957-3961

Alexandre J, Raymond E, Kaci MO, Brain EC et al (2004) Phase I and pharmacokinetic study of irofulven administered weekly or biweekly in advanced solid tumor patients. Clin Cancer Res 10:3377-3385

Alexopoulos CJ, Mims CW, Blackwell M (1996) Introductory mycology, 4th edn. Wiley, New York

Ali MR, Hoque MS, Ahmed KU, Rahman MH (2010) Effect of wheat bran supplements with sugarcane bagasse on the yield and proximate composition of Pleurotus ostreatus. Bangladesh $\mathrm{J}$ Mushroom 4:21-26

Allison AC, Eugui EM (2000) Mycophenolate mofetil and its mechanisms of action. Immunopharmacology 47:85-118

Almeida JR, Bertilsson M, Gorwa-Grauslund MF, Gorsich S, Lidén G (2009) Metabolic effects of furaldehydes and impacts on biotechnological processes. Appl Microbiol Biotechnol 82:625-638

Alpha CJ, Campos M, Jacobs-Wagner C, Strobel SA (2015) Mycofumigation by the volatile organic compound-producing fungus Muscodor albus induces bacterial cell death through DNA damage. Appl Environ Microbiol 81:1147-1156

Alves MJ, Ferreira IC, Dias JF, Teixeira V, Martins A, Pintado M (2012a) A review on antimicrobial activity of mushroom (Basidiomycetes) extracts and isolated compounds. Planta Med 78:1707-1718

Alves MJ, Ferreira IC, Martins A, Pintado M (2012b) Antimicrobial activity of wild mushroom extracts against clinical isolates resistant to different antibiotics. J Appl Microbiol 113:466-475

Ambati RR, Phang SM, Ravi S, Aswathanarayana RG (2014) Astaxanthin: sources, extraction, stability, biological activities and its commercial applications-a review. Mar Drugs 12:128 152

Ananbeh KM, Almomany AR (2005) Production of oyster mushroom Pleurotus ostreatus on olive cake agro waste. Dirasat Agric Sci 32:64-70

Ananbeh KM, Almomany AR (2008) Production of Oyster mushroom (Pleurotus ostreatus) on tomato tuff agro-waste. Dirasat Agric Sci 35:133-138

Anchana Devi A (2014) Extraction of natural dyes from fungus-an alternate for textile dyeing. J Nat Sci Res 4:1-7

Anderson PK, Cunningham AA, Patel NG, Morales FJ et al (2004) Emerging infectious diseases of plants: pathogen pollution, climate change and agro technology drivers. Trends Ecol Evol 19:535-544 
Andrady AL (2015) Persistence of plastic litter in the oceans. In: Bergmann M, Gutow L, Klages M (eds) Marine anthropogenic litter. Springer, Cham, pp 57-72

Anitha TS, Palanivelu P (2013) Purification and characterization of an extracellular keratinolytic protease from a new isolate of Aspergillus parasiticus. Protein Expr Purif 88:214-220

Anke H, Stadler M, Mayer A, Sterner O (1995) Secondary metabolites with nematicidal and antimicrobial activity from nematophaous fungi and Ascomycetes. Can J Bot 73:932-939

Ao T, Deb CR, Khruomo N (2016) Wild edible mushrooms of Nagaland, India: a potential food resource. J Exp Biol Agric Sci 4:59-65

Apostolidis C, McLeay F (2016) It's not vegetarian, it's meat-free! Meat eaters, meat reducers and vegetarians and the case of Quorn in the UK. Soc Bus 6:267-290

Arfi Y, Chevret D, Henrissat B, Berrin JG et al (2013) Characterization of salt-adapted secreted lignocellulolytic enzymes from the mangrove fungus Pestalotiopsis sp. Nat Commun 4:1810

Arisoy M (1998) Biodegradation of chlorinated organic compounds by white-rot fungi. Bull Environ Contam Toxicol 60:872-876

Ariyawansa HA, Hyde KD, Jayasiri SC, Buyck B et al (2015) Fungal diversity notes 111-252 taxonomic and phylogenetic contributions to fungal taxa. Fungal Divers 75:1-248

Armstrong DW, Brown LA (1994) Aliphatic, aromatic, and lactone compounds. Bioprocess production of flavor, fragrance, and color ingredients. Wiley, Hoboken, pp 41-94

Arora DK (2003) Handbook of fungal biotechnology, 2nd edn. Marcel Dekker Inc., New York, p 573

Arora DS, Chander M, Gill PK (2002) Involvement of lignin peroxidase manganese peroxidase and laccase in degradation and selective ligninolysis of wheat straw. Int Biodeter Biodegr 15:115-120

Ashrafi S, Helaly S, Schroers HJ, Stadler M et al (2017a) Ijuhya vitellina sp. nov., a novel source for chaetoglobosin $\mathrm{A}$, is a destructive parasite of the cereal cyst nematode Heterodera filipjevi. PLoS ONE 12:e0180032

Ashrafi S, Stadler M, Dababat AA, Richert- Poeggeler KR et al (2017b) Monocillium gamsii sp. nov. and Monocillium bulbillosum: two nematode-associated fungi parasitising the eggs of Heterodera filipjevi. MycoKeys 27:21

Ashrafi S, Knapp DG, Blaudez D, Chalot M et al (2018) Inhabiting plant roots, nematodes, and truffles-Polyphilus, a new helotialean genus with two globally distributed species. Mycologia 110:286-299

Asmelash F, Bekele T, Birhane E (2016) The potential role of arbuscular mycorrhizal fungi in the restoration of degraded lands. Front Microbiol 7:1095

Astuti M, Meliala A, Dalais FS, Wahlqvist ML (2000) Tempe, a nutritious and healthy food from Indonesia. Asia Pac J Clin Nutr 9:322-325

Atalla MM, El-khrisy EAM, Youssef YA, Mohamed AA (2011) Production of textile reddish brown dyes by fungi. Mal J Microb 7:33-40

Atkinson CJ, Fitzgerald JD, Hipps NA (2010) Potential mechanisms for achieving agricultural benefits from biochar application to temperate soils: a review. Plant Soil 337:1-18

Atlas R, Bragg J (2009) Bioremediation of marine oil spills: when and when not-the Exxon Valdez experience. Microb Biotechnol 2:213-221

Atmosukarto I, Castillo U, Hess WM, Sears J, Strobel G (2005) Isolation and characterization of Muscodor albus I-41.3 s, a volatile antibiotic producing fungus. Plant Sci 169:854-861

Auld BA (1993) Vegetable oil suspension emulsions reduce dew dependence of a mycoherbicide. Crop Prot 12:477-479
Auld BA (1997) Bioherbicides in biological control of weeds. Theory and practical application. Australian Centre for International Agricultural Research, Canberra, pp 129-134

Aust SD, Swaner PR, Stahl JD (2003) Detoxification and metabolism of chemicals by white-rot fungi. In: Zhu JJPC, Asut SD, LemleyGan AT (eds) Pesticide decontamiantion and detoxification. Oxford University Press, Washington, DC, pp 3-14

Ayob FW, Simarani K, Zainal Abidin N, Mohamad J (2017) First report on a novel Nigrospora sphaerica isolated from Catharanthus roseus plant with anticarcinogenic properties. Microb Biotechnol 10:926-932

Azren PD, Lee SY, Emang D, Mohamed R (2019) History and perspectives of induction technology for agarwood production from cultivated Aquilaria in Asia: a review. J For Res 30:1-11

Babel FJ (1953) The role of fungi in cheese ripening. Econ Bot 7:2742

Babu PD, Bhakyaraj R, Vidhyalakshmi R (2009) A low cost nutritious food "tempeh"- a review. World J Dairy Food Sci 4:22-27

Backman PA, Sikora RA (2008) Endophytes: an emerging tool for biological control. Biol Control 46:1-3

Badalyan SM, Barkhudaryan A, Rapior S (2019) Recent progress in research on the pharmacological potential of mushrooms and prospects for their clinical application. In: Agrawal DC, Dhansekaran M (eds) Medicinal mushrooms: recent progress in research and development. Publisher Springer Nature Singapore, pp 1-70. https://doi.org/10.1007/978-981-13-6382-5_1

Bagchi D, Ghosh D, Konishi T (2014) Clinical aspects of functional foods and nutraceuticals, 1st edn. CRC Press, Boca Raton, pp 51-76

Bagga J, Pramanik SK, Pandey V (2015) Production and purification of tannase from Aspergillus aculeatus using plant derived raw tannin. Int J Sci Eng Technol 4:50-55

Bai R, Zhang CC, Yin X, Wei J, Gao JM (2015) Striatoids A-F, cyathane diterpenoids with neurotrophic activity from cultures of the fungus Cyathus striatus. J Nat Prod 78:783-788

Bailey KL, Boyetchko SM, Langle T (2010) Social and economic drivers shaping the future of biological control: a Canadian perspective on the factors affecting the development and use of microbial biopesticides. Biol Control 52:221-229

Bailey KL, Pitt WM, Falk S, Derby J (2011) The effects of Phoma macrostoma on non-target plant and target weed species. Biol Control 58:379-386

Bailey KL, Falk S, Derby JA et al (2013) The effect of fertilizers on the efficacy of the bioherbicide, Phoma macrostoma, to control dandelions in turfgrass. Biol Control 65:147-151

Bailey AM, Alberti F, Kilaru S, Collins CM et al (2016) Identification and manipulation of the pleuromutilin gene cluster from Clitopilus passeckerianus for increased rapid antibiotic production. Sci Rep 6:25202

Bajaj R, Huang Y, Gebrechristos S, Mikolajczyk B et al (2018) Transcriptional responses of soybean roots to colonization with the root endophytic fungus Piriformospora indica reveals altered phenylpropanoid and secondary metabolism. Sci Rep 8(1):10227

Bakshi M, Sherameti I, Meichsner D, Thürich J et al (2017) Piriformospora indica reprograms gene expression in Arabidopsis phosphate metabolism mutants but does not compensate for phosphate limitation. Front Microbiol 8:1262

Bal C (2018) Benefits and uses of mushroom. J Bacteriol Mycol 6:155-156

Balak DMW (2015) Fumaric acid esters in the management of psoriasis. Psoriasis 5:9-23

Balakrishnan B, Karki S, Chiu SH, Kim HJ et al (2013) Genetic localization and in vivo characterization of a Monascus azaphilone pigment biosynthetic gene cluster. Appl Microbiol Biotechnol 97:6337-6345 
Baldrian P (2003) Interactions of heavy metals with white-rot fungi. Enzyme Microb 32(1):78-91

Bandara AR, Rapior S, Bhat DJ, Kakumyan P et al (2015) Polyporus umbellatus, an edible-medicinal cultivated mushroom with multiple developed health-care products as food, medicine and cosmetics: a review. Cryptogamie Mycol 36(1):3-42

Bandara AR, Karunarathna SC, Mortimer PE, Hyde KD et al (2017) First successful domestication and determination of nutritional and antioxidant properties of the red ear mushroom Auricularia thailandica (Auriculariales, Basidiomycota). Mycol Prog 16(1112):1029-1039

Banerjee A, Chatterjee K, Madras G (2014) Enzymatic degradation of polymers: a brief review. J Mater Sci Technol 30:567-573

Banik SP, Khowala S, Pal C, Mukherjee S (2015) Proteomic approaches to identify novel therapeutics and nutraceuticals from filamentous fungi. In: Bagchi D, Swaroop A, Bagchi M (eds) Genomics, proteomics and metabolomics in nutraceuticals and functional foods, 2nd edn. Wiley, New York, pp 265-295

Bano A, Hussain J, Akbar A, Mehmood K et al (2018) Biosorption of heavy metals by obligate halophilic fungi. Chemosphere 199:218-222

Banu AR, Devi MK, Gnanaprabhal GR, Pradeep BV et al (2010) Production and characterization of pectinase enzyme from Penicillium chrysogenum. Indian J Sci Technol 3:377-381

Baral HO, Weber E, Gams W, Hagedorn G, Liu B, Liu X, Marson G, Marvanová L, Stadler M, Weiß M (2018) Recommendations about generic names to be protected or suppressed in the Orbiliaceae (Orbiliomycetes). Mycol Prog 17:5-31

Baroni TJ (2017) Mushrooms of the Northeastern United States and Eastern Canada. Timber Press, Portland

Barratt SR, Ennos AR, Greenhalgh M, Robson GD et al (2003) Fungi are the predominant micro-organisms responsible for degradation of soil-buried polyester polyurethane over a range of soil water holding capacities. J Appl Microbiol 95:78-85

Barry D, Williams G, Chan C (2015) Automated analysis of filamentous microbial morphology with AnaMorf. Biotechnol Prog 31:849-852

Bashir KM, Choi JS (2017) Clinical and physiological perspectives of $\beta$-glucans: the past, present, and future. Int J Mol Sci 18:1906

Bashyal B, Wellensiek B, Ramakrishnan R, Faeth SH, Ahmad N, Gunatilaka AA (2014) Altertoxins with potent anti-HIV activity from Alternaria tenuissima QUE1Se, a fungal endophyte of Quercus emoryi. Bioorg Med Chem 22:6112-6116

Bateman B, Warner JO, Hutchinson E, Dean T et al (2004) The effects of a double blind, placebo controlled, artificial food colourings and benzoate preservative challenge on hyperactivity in a general population sample of preschool children. Arch Dis Child 89:506-511

Batra A, Saxena RK (2005) Potential tannase producers from the genera Aspergillus and Penicillium. Process Biochem 40:15531557

Becker J, Lange A, Fabarius J, Wittmann C (2015) Top value platform chemicals: bio-based production of organic acids. Curr Opin Biotechnol 36:168-175

Beetz AE, Greer L (1999) Mushroom cultivation and marketing. ATTRA, Melbourne

Bei J, Chen Z, Fu J, Jiang Z et al (2009) Structure-based fragment shuffling of two fungal phytases for combination of desirable properties. J Biotechnol 139:186-193

Belmares R, Contreras-Esquivel JC, Rodriguez-Herrera R, Coronel AR et al (2004) Microbial production of tannase: an enzyme with potential use in food industry. Lebensm Wiss Technol 37:857-864

Benjamins R, Scheres B (2008) Auxin: the looping star in plant development. Annu Rev Plant Biol 59:443-465
Benocci T, Aguilar-Pontes MV, Zhou M, Seiboth B, Vries RP (2017) Regulators of plant biomass degradation in ascomycetous fungi. Biotechnol Biofuels 10:152

Bentley R (2000) Mycophenolic acid: a one hundred year odyssey from antibiotic to immunosuppressant. Chem Rev 100:380-3826

Berrin JG, Navarro D, Couturier M, Olivé C et al (2012) Exploring the natural fungal biodiversity of tropical and temperate forests toward improvement of biomass conversion. Appl Environ Microbiol 78(18):6483-6490

Besl H, Bresinsky A (1997) Chemosystematics of Suillaceae and Gomphidiaceae (suborder Suillineae). Plant Syst Evol 206:223242

Besserer A, Puech-Pages V, Kiefer P, Gomez-Roldan V et al (2006) Strigolactones stimulate arbuscular mycorrhizal fungi by activating mitochondria. PLoS Biol 4:e226

Beyer DM (2003) Basic procedures for Agaricus mushroom growing. Pennsylvania State University, University Park, pp 1-16

Bezalel L, Hadar Y, Fu PP, Freeman JP et al (1996) Initialoxidation products in the metabolism of pyrene, anthracene, fluorene, and dibenzothiophene by the white rot fungus Pleurotus ostreatus. Appl Environ Microbiol 62:2554-2559

Bhalerao TS, Puranik P (2007) Biodegradation of organochlorine pesticide, endosulfan, by a fungal soil isolate, Aspergillus niger. Int Biodeterior Biodegrad 59:315-321

Bhat MK (2000) Cellulases and related enzymes in biotechnology. Biotechnol Adv 18:355-383

Bhuiyan SA, Croft BJ, James RS, Cox MC (2012) Laboratory and field evaluation of fungicides for the management of sugarcane smut caused by Sporisorium scitamineum in seedcane. Australas Plant Pathol 41:591-599

Bilal L, Asaf S, Hamayun M, Gul H (2018) Plant growth promoting endophytic fungi Aspergillus fumigatus TS1 and Fusarium proliferatum BRL1 produce gibberellins and regulates plant endogenous hormones. Symbiosis 76:117-127

Bilgrami KS, Sinha SP, Jeswal P (1988) Nephrotoxic and hepatotoxic effects of citrinin in mice (Mus musculus). Proc Natl Acad Sci India B 54(1):35-37

Bills GF, Gloer JB (2016) Biologically active secondary metabolites from the Fungi. Microbiol Spectr 4:VE-0001-2014

Bills GF, Martín J, Collado J, Platas G et al (2009) Measuring the distribution and diversity of antibiosis and secondary metabolites in the filamentous fungi. SIM News 59:133-147

Birch GG, Lindley MG (1985) Alcoholic beverages. Elsevier, London

Bisht S, Pandey P, Bhargava B, Sharma S et al (2015) Bioremediation of polyaromatic hydrocarbons (PAHs) using rhizosphere technology. Braz J Microbiol 46:7-21

Bitas V, McCartney N, Li N, Demers J (2015) Fusarium oxysporum volatiles enhance plant growth via affecting auxin transport and signaling. Front Microbiol 6:1248

Bitzer J, Henkel T, Nikiforov AI, Rihner MO, Henderson KA (2017a) A 90-day oral toxicity study of glycolipids from Dacryopinax spathularia in Beagle dogs. Food Chem Toxicol 109:544-551

Bitzer J, Henkel T, Nikiforov AI, Rihner MO, Leuschner J (2017b) A 90-day oral toxicity study of glycolipids from Dacryopinax spathularia in $\mathrm{CD}^{\circledR}$ rats. Food Chem Toxicol 109:569-584

Bitzer J, Henkel T, Nikiforov AI, Rihner MO, Thomas JA (2017c) Pharmacokinetics, excretion balance, and tissue distribution of [14C]-labeled glycolipids and long chain fatty acids from Dacryopinax spathularia in rats. Food Chem Toxicol 109:552568

Bitzer J, Henkel T, Nikiforov AI, Rihner MO, Herberth MT (2018) Developmental and reproduction toxicity studies of glycolipids from Dacryopinax spathularia. Food Chem Toxicol 120:430438

Biz A, Finkler ATJ, Pitol LO, Medina BS et al (2016) Production of pectinases by solid-state fermentation of a mixture of citrus 
waste and sugarcane bagasse in a pilot-scale packed-bed bioreactor. Biochem Eng J 111:54-62

Bjawa AA (2014) Sustainable weed management in conservation agriculture. Crop Prot 65:105-113

Bleve G, Grieco F, Cozzi G, Logrieco A, Visconti A (2006) Isolation of epiphytic yeasts with potential for biocontrol of Aspergillus carbonarius and $A$. niger on grape. Int $\mathrm{J}$ Food Microbiol 108:204-209

Blin K, Wolf T, Chevrette MG, Lu X et al (2017) antiSMASH 40-improvements in chemistry prediction and gene cluster boundary identification. Nucl Acids Res 45(W1):W36-W41

Blomqvist J (2011) Dekkera bruxellensis - a competitive yeast for ethanol production from conventional and non-conventional substrates. Doctoral thesis, Faculty of Natural Resources and Agricultural Sciences Department of Microbiology. Swedish University of Agricultural Sciences, Uppsala

Bluemke W, Schrader J (2001) Integrated bioprocess for enhanced production of natural flavors and fragrances by Ceratocystis moniliformis. Biomol Eng 17:137-142

Boa E (2004) Wild edible fungi: a global overview of their use and importance to people (non-wood forest products). Food \& Agriculture Organization of the United Nations, Rome

Bobeck P, Ginter E, Jurcovicova M, Kuniak L (1991) Cholesterollowering effect of the mushroom Pleurotus ostreatus in hereditary hypercholesterolemic rats. Ann Nutr Metab 35:191-195

Boecker S, Grätz S, Kerwat D, Adam L et al (2018) Aspergillus niger is a superior expression host for the production of bioactive fungal cyclodepsipeptides. Fungal Biol Biotechnol 5:7

Bonomo L, Solari E, Scopelliti R, Floriani C (2001) The $\pi$ complexation of alkali and alkaline earth ions by the use of $\mathrm{m}$ meso-Octaalkylporphyrinogen and aromatic hydrocarbons. Chem Eur J 7:1322-1332

Bonugli-Santos RC, Durrant LR, Silva M, Sette LD (2010) Production of laccase manganese peroxidase and lignin peroxidase by Brazilian marine-derived fungi. Enzyme Microb Technol 46:3237

Boubendir A (1993) Purification and biochemical evaluation of polyurethane degrading enzymes of fungal origin. Diss Abstr Int 53:4632

Bourdichon F, Casaregola S, Farrokh C, Frisvad JC et al (2012) Food fermentations: microorganisms with technological beneficial use. Int J Food Microbiol 154:87-97

Boyetchko SM, Pedersen E, Punja ZK, Reddy MS (1999) Formulations of biopesticides. In: Hall FR, Barry JW (eds) Bio pesticides: use and delivery. Methods in biotechnology, vol 5. Humana Press, Totowa, pp 487-508

Boyette CD, Templeton GE, Smith RJ Jr (1979) Control of winged water primrose (Jussiaea decurrens) and Northern joint vetch (Aeschynomene virginica) with fungal pathogens. Weed Sci 27:497-501

Bozell JJ, Petersen GR (2010) Technology development for the production of biobased products from biorefinery carbohydratesthe US Department of Energy's “Top10” revisited. Green Chem 12:539-554

Brakhage AA, Schroeckh V (2010) Fungal secondary metabolitesstrategies to activate silent gene clusters. Fungal Genet Biol 48:15-22

Brearley FQ, Saner P, Uchida A, Burslem DFRP et al (2016) Testing the importance of a common ectomycorrhizal network for dipterocarp seedling growth and survival in tropical forests of Borneo. Plant Ecol Divers 9:563-576

Brennan MA, Derbyshire E, Tiwari BK, Brennan CS (2012) Enrichment of extruded snack products with coproducts from chestnut mushroom (Agrocybe aegerita) production: interactions between dietary fiber, physicochemical characteristics, and glycemic load. J Agric Food Chem 60:4396-4401
Briggs RS, Atkins E (1966) Studies in cryptococcal fever. I. Responses to intact organisms and to a soluble agent derived from cryptococci. Yale J Biol Med 38:431-448

Brown AC, Valiere A (2004) Probiotics and medical nutrition therapy. Nutr Clin Care 7:56

Brown AG, Smale TC, King TJ, Hasenkamp R, Thompson RH (1976) Crystal and molecular structure of compactin, a new antifungal metabolite from Penicillium brevicompactum. J Chem Soc 11:1165-1170

Brown MS, Faust JR, Goldstein JL (1978) Induction of 3-hydroxy-3methylglutaryl coenzyme A reductase activity in human fibroblasts incubated with compactin (ML-236B), a competitive inhibitor of the reductase. J Biol Chem 253:1121-1128

Brueckner T, Eberl A, Heumann S, Rabe M, Guebitz GM (2008) Enzymatic and chemical hydrolysis of poly(ethylene terephthalate) fabrics. J Polym Sci A 46:6435-6443

Brugger R, Nunes CS, Hug D, Vogel K et al (2004) Characteristics of fungal phytases from Aspergillus fumigatus and Sartorya fumigate. Appl Microbiol Biotechnol 63:383-389

Brum AN, Brum AA (2017) Mushroom cultivation as an alternative to rural development in south Brazil. J Nutr Food Sci 7:57

Brundrett MC (2009) Mycorrhizal associations and other means of nutrition of vascular plants: understanding the global diversity of host plants by resolving conflicting information and developing reliable means of diagnosis. Plant Soil 320:37-77

Bruns TD, Peay KG, Boynton P, Grubisha LC et al (2009) Inoculum potential of Rhizopogon spores increases with time over the first 4-yr of a 99-yr spore burial experiment. New Phytol 181:463470

Bucher VVC, Pointing SB, Hyde KD, Reddy CA (2004) Production of wood decay enzymes, loss of mass, and lignin solubilization in wood by diverse tropical fresh water fungi. Microb Ecol 48:331-337

Bucknall RA, Moores H, Simms R, Hesp B (1973) Antiviral effects of aphidicolin, a new antibiotic produced by Cephalosporium aphidicola. Antimicrob Agents Chemother 4:294-298

Budi S, Santoso E, Wahyudi A (2010) Identification of potential types of fungi on establishment agarwood stem of Aquilaria spp. Jurnal Silvikultur Tropika 1:1-5

Bunyapaiboonsri T, Yoiprommarat S, Srisanoh U, Choowong W et al (2011) Isariotins G-J from cultures of the Lepidoptera pathogenic fungus Isaria tenuipes. Phytochem Lett 4:283-286

Busby PE, Ridout M, Newcombe G (2016) Fungal endophytes: modifiers of plant disease. Plant Mol Biol 90:645-655

Bushley KE, Raja R, Jaiswal P, Cumbie JS et al (2013) The genome of Tolypocladium inflatum: evolution, organization, and expression of the cyclosporin biosynthetic gene cluster. PLoS Genet 9: e1003496

Buza T, Mccarthy FM (2013) Functional genomics: applications to production agriculture. CAB Rev 8:1-21

Byler JW, Cobb FW, Parmenter JR (1972) Occurrence and significance of fungi inhabiting galls caused by Peridermium harknessii. Can J Bot 50:1275-1282

CPL Business Consultants (2010) The 2010 worldwide biopesticides market summary. CAB International, Wallingford

Cai J, Ma H, Huang F, Zhu D, Bi J, Ke Y, Zhang T (2013) Correlation of bevacizumab-induced hypertension and outcomes of metastatic colorectal cancer patients treated with bevacizumab: a systematic review and meta-analysis. World J Surg Oncol 11:306

Cambell I (1996) Wild yeast in brewing and distilling. In: Priest FG, Campbell I (eds) Brewing microbiology, 2nd edn. Chapman \& Hall, London, pp 193-208

Cameron DD, Leake JR, Read DJ (2006) Mutualistic mycorrhiza in orchids: evidence from plant-fungus carbon and nitrogen 
transfers in the green leaved terrestrial orchid Goodyera repens. New Phytol 171:405-416

Canete-Rodriguez AM, Santos-Duenas IM, Jimenez-Hornero JE, Ehrenreich A et al (2016) Gluconic acid: Properties, production methods and applications - an excellent opportunity for agroindustrial by-products and waste bio-valorization. Process Biochem 51:1891-1903

Cao Y, Wu SH, Dai YC (2012) Species clarification of the prize medicinal Ganoderma mushroom "Lingzhi". Fungal Divers 56:49-62

Cao CY, Zhang CC, Shi XW, Li D, Cao W, Yin X, Gao JM (2018) Sarcodonin $\mathrm{G}$ derivatives exhibit distinctive effects on neurite outgrowth by modulating NGF signaling in PC12 Cells. ACS Chem Neurosci 9(7):1607-1615

Carrasco J, Zied DC, Pardo JE, Preston GM, Pardo-Giménez A (2018) Supplementation in mushroom crops and its impact on yield and quality. AMB Express 8:146

Carrau F, Gaggero C, Aguilar PS (2015) Yeast diversity and native vigor for flavor phenotypes. Trends Biotechnol 33:148-154

Carroll G (1988) Fungal endophytes in stems and leaves-from latent pathogen to mutualistic symbiont. Ecology 69:2-9

Carroll AL, Desai SH, Atsumi S (2016) Microbial production of scent and flavor compounds. Curr Opin Biotechnol 37:8-15

Casassa LF, Sari ES (2014) Sensory and chemical effects of two alternatives of prefermentative cold soak in Malbec wines during winemaking and bottle ageing. Int J Food Sci Technol 50:10441055

Casey A, Walsh G (2004) Identification and characterization of a phytase of potential commercial interest. J Biotechnol 110:313322

Caspeta L, Castillo T, Nielsen J (2015) Modifying yeast tolerance to inhibitory conditions of ethanol production processes. Front Bioeng Biotechnol 3:184

Castro de Souza AR, Baldoni DB, Lima J et al (2016) Selection, isolation, and identification of fungi for bioherbicide production. Braz J Microbiol 48:101-108

Cavello IA, Crespo JM, García SS, Zapiola JM et al (2015) Plant growth promotion activity of keratinolytic fungi growing on a recalcitrant waste known as "Hair Waste". Biotechnol Res Int 2015:1-10

Cayrol JC, Frankowski JP, Laniece A, D'hardemare G et al (1978) Contre les nematodes en champignonnieres: mise au point d'une methode de lutte biologique a l'aide d'un Hyphomycete predateur: Arthrobotrys robusta souche antipolis (royal 300). PHM Revue Horticole 184:23-30

Ceballos I, Ruiz M, Fernández C, Peña R et al (2013) The in vitro mass-produced model mycorrhizal fungus, Rhizophagus irregularis, significantly increases yields of the globally important food security crop cassava. PLoS ONE 8(8):e70633

Cha DY, Yoo YB (1997) Cultivation of reishi (Ganoderma lucidum). Food Rev Int 13:373-378

Chadha BS, Harmeet G, Mandeep M, Saini HS, Singh N (2004) Phytase production by the thermophilic fungus Rhizomucor pusillus. World J Microbiol Biotechnol 20:105-109

Chadni Z, Rahaman MH, Jerin I, Hoque KMF, Reza MA (2017) Extraction and optimisation of red pigment production as secondary metabolites from Talaromyces verruculosus and its potential use in textile industries. Mycology 8:48-57

Chan GC, Chan WK, Sze DM (2009) The effects of $\beta$-glucan on human immune and cancer cells. J Pediatr Hematol Oncol 1:25

Chanclud E, Kisiala A, Emery NR, Chalvon V et al (2016) Cytokinin production by the rice blast fungus is a pivotal requirement for full virulence. PLoS Pathogens 12(2):e1005457

Chanclud E, Morel J (2016) Plant hormones: a fungal point of view. Mol Plant Pathol 17(8):1289-1297
Chandra P, Arora DS, Pal M, Sharma RK (2018) Antioxidant potential and extracellular auxin production by white rot fungi. Appl Biochem Biotechnol 187:531-539

Chandrasekaran G, Choi SK, Lee YC, Kim GJ, Shin HJ (2014) Oxidative biodegradation of single-walled carbon nanotubes by partially purified lignin peroxidase from Sparassis latifolia mushroom. J Ind Eng Chem 20:3367-3374

Chang ST (1980) Mushrooms as human food. Bioscience 30:399-401

Chang ST, Buswell JA (1996) Mushroom nutriceuticals. World J Microbiol Biotechnol 12:473-476

Chang S, Wasser S (2017) The cultivation and environmental impact of mushrooms. Oxford University Press, New York

Chapla D, Pandit P, Shah A (2012) Production of xylooligosaccharides from corncob xylan by fungal xylanase and their utilization by probiotics. Bioresour Technol 115:215-221

Chen MM (2000) Cultivation techniques for Dictyophora, Polyporus umbellata, and Coprinus comatus. In: Griensven V (ed) Science and cultivation of edible fungi. Balkema, Rotterdam, pp 543-548

Chen ZD, Liu QJ, Zhou CQ (1985) Microorganism and the fermentation of Pu-erh tea. Tea Sci Technol 4:4-7

Chen KK, Zhu HT, Wang D et al (2006a) Isolation and identification of Aspergillus species from the post fermentative process of $\mathrm{Pu}$ er ripe tea. Acta Bot Yunnanica 28:123-126

Chen KK, Zhang XL, Zhu HT et al (2008) The effects of Aspergillus on the post-fermentative process of Pu-er tea. Acta Bot Yunnanica 30:624-628

Chen X, Shi Q, Lin G, Guo S, Yang J (2009) Spirobisnaphthalene analogues from the endophytic fungus Preussia sp. J Nat Prod 72:1712-1715

Chen LS, Ma Y, Maubois JL, He S et al (2010) Screening for the potential probiotic yeast strains from raw milk to assimilate cholesterol. Dairy Sci Technol 90:537-548

Chen HQ, Yang Y, Jian X, Wei JH et al (2011) Comparison of compositions and antimicrobial activities of essential oils from chemically stimulated agarwood, wild agarwood and healthy Aquilaria sinensis (Lour.) Gilg trees. Molecules 16:4884-4896

Chen MC, Lai JN, Chen PC, Wang JD (2013) Concurrent use of conventional drugs with Chinese herbal products in Taiwan: a population-based study. J Tradit Complement Med 3:256-262

Chen W, He Y, Zhou Y, Shao Y et al (2015) Edible filamentous fungi from the species Monascus early traditional fermentations, modern molecular biology, and future genomics. Compr Rev Food Sci Food Saf 14:555-567

Chen K, Yao Q, Sun J, He Z et al (2016) International publication trends and collaboration performance of China in healthcare science and services research. Isr J Health Policy 5:1-15

Chen X, Sui C, Liu Y, Yang Y et al (2017) Agarwood formation induced by fermentation liquid of Lasiodiplodia theobromae, the dominating fungus in wounded wood of Aquilaria sinensis. Curr Microbiol 74:460-468

Chen X, Liu Y, Yang Y, Feng J et al (2018b) Trunk surface agarwood-inducing technique with Rigidoporus vinctus: an efficient novel method for agarwood production. PLoS ONE 13(6): $\mathrm{e} 0198111$

Chen JJ, Feng XX, Xia CY, Kong DD, Qi ZY, Liu F, Chen D, Lin FC, Zhang CL (2019) The phylogenetic position of the genus Muscodor and the description of a new Muscodor species. Mycosphere 10:187-201

Cheng CR, Yue QX, Wu ZY, Song X et al (2010) Cytotoxic triterpenoids from Ganoderma lucidum. Phytochemistry 71:1579-1585

Cheng JH, Tsai CL, Tsai CL, Lien YY (2016) High molecular weight of polysaccharides from Hericium erinaceus against amyloid beta-induced neurotoxicity. BMC Complem Altern Med 16:1-9

Chepkirui C, Stadler M (2017) The genus Diaporthe: a rich source of diverse and bioactive metabolites. Mycol Progr 16:477-494 
Chepkirui C, Richter C, Matasyoh JC, Stadler M (2016) Monochlorinated calocerins A-D and 9-oxostrobilurin derivatives from the basidiomycete Favolaschia calocera. Phytochemistry 132:95101

Chepkirui C, Cheng T, Matasyoh J, Decock C, Stadler M (2018) An unprecedented spiro [furan-2,1'-indene]-3-one derivative and other nematicidal and antimicrobial metabolites from Sanghuangporus sp. (Hymenochaetaceae, Basidiomycota) collected in Kenya. Phytochem Lett 25:141-146

Cheung PCK (1996) The hypercholesterolemic effect of two edible mushrooms-Auricularia auricula (tree-ear) and Tremella fuciformis (white jelly-leaf) in hypercholesterolemic rats. Nutr Res 16:1721-1725

Chhipa H, Chowdhary K, Kaushik N (2017) Artificial production of agarwood oil in Aquilaria sp. by fungi: a review. Phytochem Rev $16: 835-860$

Chi F, Yang P, Han F, Jing Y, Shen S (2010) Proteomic analysis of rice seedlings infected by Sinorhizobium meliloti 1021. Proteomics 10:1861-1874

Chien RC, Yang YC, Lai EI, Mau JL (2017) Anti-inflammation and lipogenic Inhibition of Taiwanofungus salmonea mycelium and Grifola frondosa fruiting body. Int J Med Mushrooms 19:629640

Chin YW, Balunas MJ, Chai HB, Kinghorn AD (2006) Drug discovery from natural sources. AAPS J 8:239-253

Choi JM, Han SS, Kim HS (2015) Industrial applications of enzyme biocatalysis: current status and future aspects. Biotechnol Adv 33:1443-1454

Chowdhury M, Hussain MD, Chung SO, Kabir E, Rahman A (2016) Agarwood manufacturing: a multidisciplinary opportunity for economy of Bangladesh-a review. Agric Eng Int 18(3):171178

Christen P, López-Munguía A (1994) Enzymes and food flavor-a review. Food Biotechnol 8:167-190

Christoph N, Bauer-Christoph C (2007) Flavour of spirit drinks: raw materials, fermentation, distillation, and ageing. In: Berger RG (ed) Flavours and fragrances. Springer, Berlin, pp 219-239

Christoph N, Geßner M, Simat TJ, Hoenicke K (1999) Off-flavor compounds in wine and other food products formed by enzymatical, physical, and chemical degradation of tryptophan and its metabolites. In: Huether G, Kochen W, Simat TJ, Steinhart H (eds) Tryptophan, serotonin, and melatonin. Advances in experimental medicine and biology, vol 467. Springer, Boston

Chugh S, Guha S, Rao IU (2009) Micropropagation of orchids: a review on the potential of different explants. Sci Hortic 122:507520

Clay K, Shearin ZRC, Bourke KA, Bickford WA, Kowalski KP (2016) Diversity of fungal endophytes in non-native Phragmites australis in the Great Lakes. Biol Invasions 18:2703-2716

Cocchi L, Vescovi L, Petrini LE, Petrini O (2006) Heavy metals in edible mushrooms in Italy. Food Chem 98:277-284

Cock MJW, van Lenteren J, Brodeur J, Barratt B, Bigler F et al (2010) Do new access and benefit sharing procedures under the convention on biological diversity threaten the future of biological control? Biol Control 55:199-218

Coello-Castillo MM, Sánchez JE, Royse DJ (2009) Production of Agaricus bisporus on substrates pre-colonized by Scytalidium thermophilum and supplemented at casing with protein-rich supplements. Bioresour Technol 100:4488-4492

Colak M (2004) Temperature profiles of Agaricus bisporus in composting stages and effects of different composts formulas and casing materials on yield. Afr J Biotechnol 3:456-462

Colmenares-Cruz S, Sánchez JE, Valle-Mora J (2017) Agaricus bisporus production on substrates pasteurized by self-heating. AMB Express 7:135
Commercial Industrial Research Institute (CIRI) (2017) China's edible mushroom industry perspective report (2017年中国食用 菌产业市场前景研究报告)

Compant S, Clément C, Sessitsch A (2010) Plant growth-promoting bacteria in the rhizo-and endosphere of plants: their role, colonization, mechanisms involved and prospects for utilization. Soil Biol Biochem 42:669-678

Cooper MA, Shlaes D (2011) Fix the antibiotics pipeline. Nature 472:32

Corner EJH (1989) Beihefte zur Nova Hedwigia, Heft 96: Ad Polyporaceas V. Stuttgart, Berlin, pp 62-68

Corran HS (1975) A history of brewing. Newton Abbot, David \& Charles, London

Costa MAF, Peralta RM (1999) Production of lipase by soil fungi and partial characterization of lipase from a selected strain (Penicillium wortmannii). J Basic Microbiol 39:11-15

Courty PE, Buée M, Diedhiou AG, Frey-Klett P et al (2010) The role of ectomycorrhizal communities in forest ecosystem processes: new perspectives and emerging concepts. Soil Biol Biochem 42:679-698

Couturier M, Navarro D, Olivé C, Chevret D et al (2012) Postgenomic analyses of fungal lignocellulosic biomass degradation reveal the unexpected potential of the plant pathogen Ustilago maydis. BMC Genomics 13:57-70

Couturier M, Ladevèze S, Sulzenbacher G, Ciano L et al (2018) Lytic xylan oxidases from wood-decay fungi unlock biomass degradation. Nat Chem Biol 14:306-310

Crabbe JR, Campbell JR, Thompson L, Walz SL, Schultz WW (1994) Biodegradation of a colloidal ester-based polyurethane by soil fungi. Int Biodeterior Biodegrad 33:103-113

Crafts CB, Miller CO (1974) Detection and identification of cytokinins produced by mycorrhizal fungi. Plant Physiol 54:586-588

Cram MM, Dumroese RK (2012) Mycorrhizae in forest tree nurseries. For Nurs Pests 20:1-9

Crawford RL (2006) Bioremediation. In: Dworkin M, Falkow S, Rosenberg E, Schleifer KH, Stackebrandt E (eds) The prokaryotes. Springer, New York

Croft BJ, Berding N, Cox MC, Bhuiyan S, Bruce RC (2008) Breeding smut-resistant sugarcane varieties in Australia: progress and future directions. In: Proceedings of the 2008 conference of the Australian Society of Sugar Cane Technologists, Townsville, QLD, pp 125-134

Crosetto N, Mitra A, Silva MJ, Bienko M et al (2013) Nucleotideresolution DNA double-strand breaks mapping by next-generation sequencing. Nat Methods 10:361-365

Cui L, Liu QH, Wang HX, Ng TB (2007) An alkaline protease from fresh fruiting bodies of the edible mushroom Pleurotus citrinopileatus. Appl Microbiol Biotechnol 75:81-85

Cui J, Wang C, Guo S, Yang L et al (2013) Evaluation of fungusinduced agilawood from Aquilaria sinensis in China. Symbiosis 60:37-44

Cullen JM, Delfosse ES (1985) Echium plantagineum: catalyst for conflict and change in Australia. In: Proceedings of the sixth international symposium on biological control of weeds. Agriculture Canada, Ottawa, pp 249-252

Cullen JM, Kable PF, Catt M (1973) Epidemic spread of rust imported for biological control. Nature (Lond) 244:462-464

Culliney $T$ (2014) Crop losses to arthropods. Integrated pest management: pesticide problems 3:201-225

Currie JN (1917) The citric acid fermentation of Aspergillus niger. J Biol Chem 31:15-37

Custodio D, Cristopher J (2004) Coco lumber sawdust, Mushroom growing handbook 1, Oryter mushroom cultivation. Oxford University Press, Washington, DC, pp 3-14 
Dadi AP, Varanasi S, Schall CA (2006) Enhancement of cellulose saccharification kinetics using an ionic liquid pretreatment step. Biotechnol Bioeng 95:904-910

Dai YC, Yang ZL, Cui BK, Yu CJ, Zhou LW (2009) Species diversity and utilization of medicinal mushrooms and fungi in China. Int $\mathrm{J}$ Med Mushrooms 11:287-302

Dai C, Tian L, Zhao Y, Chen Y, Xie H (2010a) Degradation of phenanthrene by the endophytic fungus Ceratobasidum stevensii found in Bischofia polycarpa. Biodeg 21:245-255

Dai YC, Zhou LW, Yang ZL, Wen HA (2010b) A revised checklist of edible fungi in China. Mycosystema 29:1-21 (In Chinese)

Dai YC, Zhou LW, Hattori T, Cao Y et al (2017) Ganoderma lingzhi (Polyporales, Basidiomycota): the scientific binomial for the widely cultivated medicinal fungus Lingzhi. Mycol Progress 16:1051-1055

Dalong M, Luhe W, Guoting Y, Liqiang M, Chun L (2011) Growth response of Pinus densifilosa seedling inoculated with three indigenous ectomycprrhizal fungi in combination. $\mathrm{Br}$ Med $\mathrm{J}$ 42:1197-1203

Daranagama DA, Hyde KD, Sir EB, Thambugala KM et al (2018) Towards a natural classification and backbone tree for Graphostromataceae, Hypoxylaceae, Lopadostomataceae and Xylariaceae. Fungal Divers 88:1-165

Das S, Rosazza JPN (2006) Microbial and enzymatic transformations of flavonoids. J Nat Prod 69:499-508

Datta S, Holmes B, Park JI, Chen Z et al (2010) Ionic liquid tolerant hyperthermophilic cellulases for biomass pretreatment and hydrolysis. Green Chem 12:338-345

Dauda WP, Alao SEL, Zarafi AB, Alabi O (2018) First Report of dieback disease of onion (Allium cepa L.) induced by Fusarium equiseti (Mart) Sacc in Nigeria. Inter J Plant Soil Sci 21:1-8

Davidson P, Taylor T, Schmidt S (2013) Chemical preservatives and natural antimicrobial compounds. In: Doyle M, Buchanan $\mathrm{R}$ (eds) Food microbiology. ASM Press, Washington, DC, pp 765801

de Alencar Guimaraes NCA, Sorgatto M, Peixoto-Nogueira SC, Betini JHA et al (2013) Bioprocess and biotechnology: effect of xylanase from Aspergillus niger and Aspergillus flavus on pulp biobleaching and enzyme production using agroindustrial residues as substrate. SpringerPlus 2:380

de Capdeville G, Souza MT Jr, Santos JRP, de Paula MS et al (2007) Selection and testing of epiphytic yeasts to control anthracnose in post-harvest of papaya fruit. Sci Hortic-amsterdam 111:179 185

de Carvalho MP, Gulotta G, do Amaral MW, Lünsdorf H et al (2016) Coprinuslactone protects the edible mushroom Coprinus comatus against biofilm infections by blocking both quorum sensing and Mur A. Environ Microbiol 18:4254-4264

De Faria MR, Wraight SP (2007) Mycoinsecticides and mycoacaricides: a comprehensive list with worldwide coverage and international classification of formulation type. Biol Control 43:237-256

De Lima CJB, Cortezi M, Lovaglio RB, Ribeiro EJ et al (2008) Production of rennet in submerged fermentation with the filamentous fungus Mucor miehei NRRL 3420. World Appl Sci J 4:578-585

de Oliveira LP, Rossi MJ, Júnior AF, Filho GNS, de Oliveira VL (2006) Viability and infectivity of an ectomycorrhizal inoculum produced in an airlift bioreactor and immobilized in calcium alginate. Braz J Microbiol 37:251-255

De Silva DD, Rapior S, Hyde KD, Bahkali AH (2012) Medicinal mushrooms in prevention and control of diabetes mellitus. Fungal Divers 56:1-29

De Silva DD, Rapior S, Sudarman E, Stadler M et al (2013) Bioactive metabolites from macrofungi: ethnopharmacology, biological activities and chemistry. Fungal Divers 62:1-40
De Silva NI, Lumyong S, Hyde KD, Bulgakov T et al (2016) Mycosphere essays 9: defining biotrophs and hemibiotrophs. Mycosphere 7:545-559

de Souza WR, de Gouvea PF, Savoldi M, Malavazi I et al (2011) Transcriptome analysis of Aspergillus niger grown on sugarcane bagasse. Biotechnol Biofuels 4:40

De Waard MA, Georgopoulos SG, Hollomon DW, Ishii H et al (1993) Chemical control of plant diseases: problems and prospects. Annu Rev Phytopathol 31:403-421

Dearnaley JD (2007) Further advances in orchid mycorrhizal research. Mycorrhiza 17:475-486

Dearnaley JWD, Perotto S, Selosse MA (2016) Structure and development of orchid mycorrhizas. Molecular mycorrhizal symbiosis (Chap. 5). Wiley, Hoboken, pp 63-86

Degenkolb T, Vilcinskas A (2016) Metabolites from nematophagous fungi and nematicidal natural products from fungi as alternatives for biological control. Part II: metabolites from nematophagous basidiomycetes and non-nematophagous fungi. Appl Microbiol Biotechnol 100:3813-3824

Del-Cid A, Gil-Durán C, Vaca I, Rojas-Aedo JF, García-Rico RO, Levicán G, Chávez R (2016) Identification and functional analysis of the mycophenolic acid gene cluster of Penicillium roqueforti. PloS One 11(1):e0147047

Delaye L, Guzman GG, Heil M (2013) Endophytes versus biotrophic and necrotrophic pathogens are fungal life-styles evolutionarily stable traits? Fungal Divers 60:125-135

Delclos KB, Tarpley WG, Miller EC (1984) 4-Aminoazobenzene and $\mathrm{N}$, N-dimethyl-4-aminoazobenzene as equipotent hepatic carcinogens in male $\mathrm{C} 57 \mathrm{BL} / 6 \mathrm{XC} 3 \mathrm{H} / \mathrm{He} \mathrm{F} 1$ mice and characterization of $\mathrm{N}$-(deoxyguanosine-8-yl)-4-aminoazobenzene as the major persistent hepatic DNA-bound dye in these mice. Cancer Res 44:2540-2550

Denning DW (2002) Echinocandins: a new class of antifungals. J Antimicrob Chemother 49:889-891

Denny A, Aisbitt B, Lunn J (2008) Mycoprotein and health. Nutr Bull 33:298-310

Devinar G, Llanes A, Masciarelli O, Luna V (2013) Different relative humidity conditions combined with chloride and sulfate salinity treatments modify abscisic acid and salicylic acid levels in the halophyte Prosopis strombulifera. Plant Growth Regul 70:247256

Dhiman S, Mukherjee G, Kumar A, Mukherjee P et al (2017) Fungal tannase: recent advances and industrial applications. In: Satyanarayana T, Deshmukh S, Johri B (eds) Developments in fungal biology and applied mycology. Springer, Singapore, pp 295-313

Dikshit R, Tallapragada P (2013) Comparative study of Monascus sanguineus and Monascus purpureus for red pigment poduction under stress condition. Int Food Res J 20:1235-1238

Dilokpimol A, Mäkelä MR, Cerullo G, Zhou M et al (2018) Fungal glucuronoyl esterases: genome mining based enzyme discovery and biochemical characterization. New Biotechnol 40:282-287

Dinis MJ, Bezerra RMF, Nunes F, Dias AA (2009) Modification of wheat straw lignin by solid state fermentation with white-rot fungi. Bioresour Technol 100:4829-4835

Djian C, Pijarowski L, Ponchet M, Arpin N et al (1991) Acetic-acid a selective nematocidal metabolite from culture filtrates of Paecilomyces lilacinus (Thom) Samson and Trichoderma longibrachiatum Rifai. Nematologica 37:101-112

Doi Y (1990) Microbial polyesters. VCH Publication, New York, p p108

Dong ZM, Zhang WW, Zhang Y, Zhang XL et al (2016) Identification and characterization of novel chitin-binding proteins from the larval cuticle of silkworm, Bombyx mori. J Proteome Res 15:1435-1445

Downey MO, Dokoozlian NK, Krstic MP (2006) Cultural practice and environmental impacts on the flavonoid composition of 
grapes and wine: a review of recent research. Am J Enol Viticult 57:257-268

Dreyfuss DH, Kelleher CA, Jones JF, Gelfand EW (1996) EpsteinBarr virus infection of $t$ cells: implications for altered t-lymphocyte activation, repertoire development and autoimmunity. Immunol Rev 152:89-110

Droby S, Wisniewski M, Macarisin D, Wilson C (2009) Twenty years of postharvest biocontrol research: is it time for a new paradigm? Postharvest Biol Technol 52:137-145

Druzhinina IS, Kubicek CP (2017) Genetic engineering of Trichoderma reesei cellulases and their production. Microbial Biotechnol 10:1485-1499

Du M, Huang S, Wang J (2014) The volatiles from fermentation product of Tuber formosanum. Open J For 4:426-429

Dubey RC, Maheshwari DK (2008) A textbook of microbiology. S. Chand \& Company Ltd. India, New Delhi

Duke SO (2012) Why have no new herbicide modes of action appeared in recent years? Pest Manag Sci 68:5022-5512

Dulmer KM, LeDuc SD, Horton TR (2014) Ectomycorrhizal inoculum potential of northeastern US forest soils for American chestnut restoration: results from field and laboratory bioassay. Mycorrhiza 24:65-74

Dupont J, Dequin S, Giraud T, Le Tacon F, Marsit S, Ropars J, Richard F, Selosse MA (2016) Fungi as a source of food. Spectrum 5:1-22

Durán N, Esposito E (2000) Potential applications of oxidative enzymes and phenoloxidase-like compounds in wastewater and soil treatment: a review. Appl Catal B 28:83-99

Eckert JW, Ogawa JM (1988) The chemical control of postharvest diseases: deciduous fruits, berries, vegetables and root/tuber crops. Annu Rev Phytopathol 26:433-469

Economist.com (2017) Daily chart-around the world, beer consumption is falling. https://www.economist.com/graphic-detail/ 2017/06/13/around-the-world-beer-consumption-is-falling

Ehsanifard Z, Mir-Mohammadrezaei F, Safarzadeh A, GhobadNejhad M (2017) Aqueous extract of Inocutis levis improves insulin resistance and glucose tolerance in high sucrose-fed Wistar rats. J Herbmed Pharmacol 6:160-164

Eik LF, Naidu M, David P, Wong KH (2012) Lignosus rhinocerus (Cooke) Ryvarden: a medicinal mushroom.that stimulates neurite outgrowth in PC-12 cells. Evid Based Complement Alternat Med 2012:320308

Eilenberg J, Hajek A, Lomer C (2001) Suggestions for unifying the terminology in biological control. Biocontrol 46:387-400

Ek-Ramos MJ, Zhou W, Valencia CU, Antwi JB (2013) Spatial and temporal variation in fungal endophyte communities isolated from cultivated cotton (Gossypium hirsutum). PLoS ONE 8:1-13

Ekor M (2014) The growing use of herbal medicines: issues relating to adverse reactions and challenges in monitoring safety. Front Pharmacol 4:177

Ekwe E, Morgenstern I, Tsang A, Storms R, Powlowski J (2013) Non-hydrolytic cellulose active proteins: research progress and potential application in biorefineries. Ind Biotechnol 9:123-131

El Morsy EM (2004) Evaluation of micro fungi for the biological control of water hyacinth in Egypt. Fungal Divers 16:35-51

El-Ghonemy DH, El-Gamal MS, Tantawy AE, Ali TH (2017) Extracellular alkaline lipase from a novel fungus Curvularia sp. DHE 5: optimisation of physicochemical parameters partial purification and characterization. Food Technol Biotechnol $55: 206-217$

El-Sayed W (2005) Biological control of weeds with pathogens: current status and future trends. Z Pflanzenkrankh Pflanzenschutz 112:209-221

El-Shaikh KAA, Mohammed MS (2009) Enhancing fresh and seed yield of okra and reducing chemical phosphorus fertilizer via using VA-mycorrhizal inoculants. World J Agric Sci 5(S):810 818

Eleazar ME, Dendooven L, Magaña IP, Parra R, De la Torre M (2000) Optimization of gibberellic acid production by immobilized Gibberella fujikuroi, mycelium in fluidized bioreactors. J Biotechnol 76:147-155

Elgharbawy AA, Alam MZ, Moniruzzaman M, Goto M (2016) Ionic liquid pre-treatment as emerging approaches for enhanced enzymatic hydrolysis of lignocellulosic biomass. Biochem Eng J 109:252-267

Ellis MB (1976) More dematiaceous hyphomycetes. Commonwealth Mycological Institute, Kew

Elsworth JF, Grove JF (1980) Cyclodepsipeptides from Beauveria bassiana. Part 2. Beauverolides A to $\mathrm{F}$ and their relationship to isarolide. J Chem Soc Perkin Trans 1:1795-1799

Endo A, Kuroda M, Tsujita Y (1976) ML-236A, ML-236B, and ML236C, new inhibitors of cholesterogenesis produced by Penicillium citrinum. J Antibiotics 29:1346-1348

El Enshasy HA, El Marzugi NA, Elsayed EA, Ling OM (2018) Medical and cosmetic applications of fungal nanotechnology: production, characterization, and bioactivity. In: Prasad R et al (eds) Fungal nanobionics: principles and applications. Springer, Singapore, pp 21-59

El Sohaimy S (2012) Functional foods and nutraceuticals-modern approach to food science. World Appl Sci J 20:691-708

Emsa-art A, Watthana S, Naradisorn M, Wongkham S, Nontachaiyapoom S (2018) Phalaenopsis Sogo Yukidian Effect of mycorrhizal inoculation on resistance against Dickeya sp. in Phalaenopsis Sogo Yukidian. The 10th Science Research Conference. pp. 279-292

Epinette WW, Parker CM, Jones EL, Greist MC (1987) Mycophenolic acid for psoriasis. A review of pharmacology, long-term efficacy, and safety. J Am Acad Dermatol 17:962-971

Ergun SO, Urek RO (2017) Production of ligninolytic enzymes by solid state fermentation using Pleurotus ostreatus. Ann Agrar Sci 15:273-277

Espiau C, Riviere D, Burdon JJ et al (1998) Host-pathogen diversity in a wild system, Chondrilla juncea-Puccinia chondillina. Oecologia 113:133-139

Espinosa-Valdemar RM, Turpin-Marion S, Delfín-Alcalá I, VázquezMorillas A (2011) Disposable diapers biodegradation by the fungus Pleurotus ostreatus. Waste Manag 31:1683-1688

European Commission (2014) Commission Regulation (EU) No 212/2014 of 6 March 2014 amending Regulation (EC) No $1881 / 2006$ as regards maximum levels of the contaminant citrinin in food supplements based on rice fermented with red yeast Monascus purpureus. http://eur-lex.europa.eu/legal-con tent/EN/TXT/?uri=OJ:L:2014:067:TOC. Accessed 22 Jan 2019

Evans HC (1998) The safe use of fungi for biological control of weeds. Phytoprotection 79:67-74

Evans HC, Seier MK, Derby JA et al (2013) Tracing the origins of White Tip disease of Cirsium arvense and its causal agent, Phoma macrostoma. Weed Res 53:42-52

Ezra D, Hess WM, Strobel GA (2004) New endophytic isolates of Muscodor albus, a volatile antibiotic producing fungus. Microbiology 150:4023-4031

Fabre CE, Blanc PJ, Goma G (1996) Production of benzaldehyde by several strains of Ischnoderma benzoinum. Sci Aliment 16:61-68

Facchini FDA, Vici AC, Pereira MG, Jorge JA, Polizeli TM (2015) Enhanced lipase production of Fusarium verticillioides by using response surface methodology and wastewater pretreatment application. J Biochem Tech 6:996-1002

Fahad S, Hussain S, Bano A, Saud S et al (2014) Potential role of phytohormones and plant growth-promoting rhizobacteria in abiotic stresses: consequences for changing environment. Environ Sci Pollut Res 22:4907-4921 
Fahad S, Bajwa AA, Nazir U, Anjum SA et al (2017) Crop production under drought and heat stress: plant responses and management options. Front Plant Sci 8:1147

Fan YM, Xu MY, Wang LY, Zhang Y et al (1989) The effect of edible black tree fungus (Auricularia auricula) on experimental atherosclerosis in rabbits. Chin Med J 102:100-105

Fang L, Liu J (2009) First synthesis of racemic concentricolide, an anti-HIV-1 agent isolated from the fungus Daldinia concentrica. Heterocycles 78(8):2107-2113

Fantozzi F, Bartocci P, D'Alessandro B, Testarmata F, Fantozzi P (2015) Carbon footprint of truffle sauce in central Italy by direct measurement of energy consumption of different olive harvesting techniques. J Clean Prod 87:188-196

Farag AM, Hassan MA (2004) Purification, characterization and immobilization of a keratinase from Aspergillus oryzae. Enzyme Microb Technol 34:85-93

Fedorak PM, Westlake DWS (1981) Microbial degradation of aromatics and saturates in Prudhoe Bay crude oil as determined by glass capillary gas chromatography. Can J Microbiol 27:432443

Fedoroff NV, Battisti DS, Beachy RN, Cooper PJ et al (2010) Radically rethinking agriculture for the 21 st century. Science 5967:833-834

Feldbrügge M, Kellner R, Schipper K (2013) The biotechnological use and potential of plant pathogenic smut fungi. Appl Microbiol Biotechnol 97:3253-3265

Feofilova EP, Tereshina VM, Memorskaya AS, Dul'kin LM, Goncharov NG (2006) Fungal lycopene: the biotechnology of its production and prospects for its application in medicine. Microbiology 75:629-633

Fernandez-Luqueno F, Valenzuela-Encinas C, Marsch R et al (2010) Microbial communities to mitigate contamination of PAHs in soil-possibilities and challenge: a review. Environ Sci Pollut Res 18:12-30

Finnigan TJA (2011) Mycoprotein: origins, production and properties. Woodhead Publishing, In Handbook of Food Proteins, pp 335-352

Finnigan T, Needham L, Abbott C (2017) Mycoprotein: a healthy new protein with a low environmental impact. In: Finnigan T, Needham L, Abbott C (eds) Sustainable protein sources. Academic Press, New York, pp 305-325

Fiori-Tutida ACG, Schwan-Estrada KRF, Stangarlin JR, Pascholati SF (2007) Extracts of Lentinula edodes and Agaricus blazei on Bipolaris sorokiniana and Puccinia recondita f. sp. tritici, in vitro. Summa Phytopathol 33:287-289

Fisch KM, Bakeer W, Yakasai AA, Song Z et al (2011) Rational domain swaps decipher programming in fungal highly reducing polyketide synthases and resurrect an extinct metabolite. J Am Chem Soc 133:16635-16641

Fischer U, Strasser M, Gutzler K (2000) Impact of fermentation technology on the phenolic and volatile composition of German red wines. Int J Food Sci Technol 35:81-94

Flykt E, Timonen S, Pennanen T (2008) Variation of ectomycorrhizal colonisation in Norway spruce seedlings in Finnish forest nurseries. Silva Fenn 42:571-585

Fochi V, Falla N, Girlanda M, Perotto S, Balestrini R (2017) Cellspecific expression of plant nutrient transporter genes in orchid mycorrhizae. Plant Sci 263:39-45

Food and Agricultural Organization of the United Nations and World Health Organization (2001) Health and nutritional properties of probiotics in food including powder milk with live lactic acid bacteria. World Health Organization. http://www.who.int/food safety/publications/fs_management/en/probiotics.pdf

Forbes RJ (1970) A short history of the art of distillation. Brill, Leiden, E. J, p 405
Fox PF, Guinee TP, Cogan TM, McSweeney PL (2017) Fundamentals of cheese science. Springer, New York

Francia C, Rapior S, Courtecuisse R, Siroux Y (1999) Current research findings on the effect of selected mushrooms on cardiovascular diseases. Int J Med Mushrooms 1:169-172

Friedman ND, Temkin E, Carmeli Y (2016) The negative impact of antibiotic resistance. Clin Microbiol Infect 22:416-422

Friedrich J, Gradišar H, Vrecl M, Pogačnik A (2005) In vitro degradation of porcine skin epidermis by a fungal keratinase of Doratomyces microsporus. Enzyme Microb Technol 36:455-460

Fu L, Yu M (2005) New progress in Fuling research. Xinjiang Tradit Chin Med 23:79-83 (in Chinese)

Fujioka K, Shimizu N, Manome Y, Ikeda K et al (2013) Discrimination method of the volatiles from fresh mushrooms by an electronic nose using a trapping system and statistical standardization to reduce sensor value variation. Sensors 13:1553215534

Förster A, Aurich A, Mauersberger S, Barth G (2007) Citric acid production from sucrose using a recombinant strain of the yeast Yarrowia lipolytica. Appl Microbiol Biotechnol 75:1409-1417

GBD (2015) Mortality and Causes of Death Collaborators (2016) Global, regional, and national life expectancy, all-cause mortality, and cause-specific mortality for 249 causes of death, 1980 2015: a systematic analysis for the Global Burden of Disease Study 2015. Lancet 388(10053):1459-1544

Gadermaier G, Hauser M, Ferreira F (2014) Allergens of weed pollen: an overview on recombinant and natural molecules. Methods 66:55-66

Gan P, Ikeda K, Irieda H et al (2013) Comparative genomic and transcriptomic analyses reveal the hemibiotrophic stage shift of Colletotrichum fungi. New Phytol 197:1236-1249

Ganassi S, Moretti A, Maria Bonvicini Pagliai A, Logrieco A, Agnese Sabatini M (2002) Effects of beauvericin on Schizaphis graminum (Aphididae). J Invert Pathol 80:90-96

Gao D, Du L, Yang J, Wu WM, Liang H (2010) A critical review of the application of white rot fungus to environmental pollution control. Crit Rev Biotechnol 30:70-77

Gao J, Kim JS, Terziev N, Cuccui I, Daniel G (2018) Effect of thermal modification on the durability and decay patterns of hardwoods and softwoods exposed to soft rot fungi. Int Biodeterior Biodegradation 127:35-45

García-Estrada C, Martín JF (2016) Biosynthetic gene clusters for relevant secondary metabolites produced by Penicillium roqueforti in blue cheeses. Appl Microbiol Biotechnol 100:8303-8313

García-Mena J, Cano-Ramirez C, Garibay-Orijel C, Ramirez-Canseco S, Poggi-Varaldo HM (2007) A PCR method for the detection and differentiation of Lentinus edodes and Trametes versicolor in defined-mixed cultures used for wastewater treatment. Appl Microbiol Biotechnol 67:524-531

Garodia S, Naidu P, Nallanchakravarthula S (2017) QUORN: an anticipated novel protein source. IJSRST 3:2395-6011

Gaskin JW, Speir RA, Harris K, Das KC et al (2010) Effect of peanut hull and pine chip biochar on soil nutrients, corn nutrient status, and yield. Agron J 102:623-633

Gaunt JL, Lehmann J (2008) Energy balance and emissions associated with biochar sequestration and pyrolysis bioenergy production. Environ Sci Technol 42:4152-4158

Gerstein HC, Miller ME, Genuth S, Ismail-Beigi F et al (2011) Longterm effects of intensive glucose lowering on cardiovascular outcomes. N Engl J Med 364:818-828

Geyer R, Jambeck JR, Law KL (2017) Production, use, and fate of all plastics ever made. Sci Adv 3:e1700782

Ghorai S, Banik SP, Verma D, Chowdhury S et al (2009) Fungal biotechnology in food and feed processing. Food Res Int 42:577-587 
Giavasis I (2014) Bioactive fungal polysaccharides as potential functional ingredients in food and nutraceuticals. Curr Opin Biotechnol 26:162-173

Giavasis I, Biliaderis C (2006) Microbial polysaccharides. In: Biliaderis C, Izydorczyk M (eds) Functional food carbohydrates. CRC Press, Boca Raton, pp 167-214

Gibson IAS (1977) The role of fungi in the origin of oleoresin deposits (agaru) in the wood of Aquilaria agallocha Roxb. Bano Biggyan Patrika 6:16-26

Gibson B, Liti G (2015) Saccharomyces pastorianus: genomic insights inspiring innovation for industry. Yeast 32:17-27

Gil-Ramírez A, Caz V, Smiderle FR, Martin-Hernandez R et al (2016) Water-soluble compounds from Lentinula edodes influencing the HMG-CoA reductase activity and the expression of genes involved in the cholesterol metabolism. J Agric Food Chem 64:1910-1920

Gilbert FA, Robinson RF (1957) Food from fungi. Econ Bot 11:126145

Gill M (1994) Pigments of fungi (Macromycetes). Nat Prod Rep 11:67-90

Gill M (2003) Pigments of fungi (Macromycetes). Nat Prod Rep 20:615-639

Gill M, Steglich W (1987) Pigments of fungi (Macromycetes). In: Progress in the Chemistry of Organic Natural Products 1987 (pp. 1-297). Springer, Vienna

Gladden JM, Park JI, Bergmann J, Reyes-Ortiz V et al (2014) Discovery and characterization of ionic liquid-tolerant thermophilic cellulases from a switchgrass-adapted microbial community. Biotechnol Biofuels 7:15

Gmose R, Ferreira JA, Lundin M, Taherzadeh MJ, Lennartsson PR (2018) Pigment production by the edible filamentous fungus Neurospora intermedia. Fermentation 4:1-15

Goates BJ, Mercier J (2009) Effect of biofumigation with volatiles from Muscodor albus on the viability of Tilletia spp. teliospores. Can J Microbiol 55:203-206

Godtfredsen WO, Jahnsen S, Lorck H (1962) Fusidic acid: a new antibiotic. Nature 193:987

Goetten LC, Moretto G, Stürmer SL (2016) Influence of arbuscular mycorrhizal fungi inoculum produced on-farm and phosphorus on growth and nutrition of native woody plant species from Brazil. Acta Bot Bras 30:9-16

Goffeau A, Barrell BG, Bussey H, Davis RW et al (1996) Life with 6000 genes. Science 274:563-567

Goksungur Y, Mantzouridou F, Roukas T (2002) Optimization of the production of $\beta$-carotene from molasses by Blakeslea trispora: a statistical approach. J Chem Technol Biotechnol 77:933-943

Goldammer T (2008) The brewer's handbook: the complete book to brewing beer. Apex Publishers, Clifton

Goldberg I, Rokem JS, Pines O (2006) Organic acids: old metabolites, new themes. J Chem Technol Biotechnol 81:1601-1611

Gong ZL, Watanabe N, Yagi A (1993) Compositional change of Puerh tea during processing. Biosci Biotechnol Biochem 57:17451746

Gonzalez-Chavez MDCA, Torres-Cruz TJ, Sanchez SA, CarrilloGonzalez R et al (2018) Microscopic characterization of orchid mycorrhizal fungi: Scleroderma as a putative novel orchid mycorrhizal fungus of Vanilla in different crop systems. Mycorrhiza 28(2):147-157

González MC, Anaya AL, Glenn AE, Macías-Rubalcava ML et al (2009) Muscodor yucatanensis, a new endophytic ascomycete from Mexican chakah, Bursera simaruba. Mycotaxon 110:363372

González Matute R, Figlas D, Curvetto N (2011) Agaricus blazei production on non-composted substrates based on sunflower seed hulls and spent oyster mushroom substrate. World J Microbiol Biotechnol 27:1331-1339
Gopal M, Gupta A (2016) Microbiome selection could spur nextgeneration plant breeding strategies. Front Microbiol 7:1971

Goto CE, Barbosa EP, Kistner LC, Moreira FG et al (1998) Production of amylase by Aspergillus fumigatus utilizing a-methyl-D-glycoside a synthetic analogue of maltose as substrate. FEMS Microbiol Lett 167:139-143

Gottshall CB, Cooper M, Emery SM (2017) Activity, diversity and function of arbuscular mycorrhizae vary with changes in agricultural management intensity. Agric Ecosyst Environ 241:142-149

Goulet C, Mageroy MH, Lam NB, Floystad A et al (2012) Role of an esterase in flavor volatile variation within the tomato clade. Proc Natl Acad Sci USA 109:19009-190014

Govinda Rajulu MB, Lai LB, Murali TS, Gopalan V, Suryanarayanan TS (2014) Several fungi from fire-prone forests of southern India can utilize furaldehydes. Mycol Prog 13:1049-1056

Gowdu BJ, Balasubramanian R (1988) Role of phylloplane microorganisms in the biological control of foliar plant diseases. J Plant Dis Protect 95:310-331

Grabski AC, Grimek HJ, Burgess RR (1998) Immobilization of manganese peroxidase from Lentinula edodes and its biocatalytic generation of Mn-III-chelate as a chemical oxidant of chlorophenols. Biotechnol Bioeng 60:204-215

Gradisar H, Kern S, Friedrich J (2000) Keratinase of Doratomyces microsporus. Appl Microbiol Biotechnol 53:196-200

Gray AM, Flatt PR (1998) Insulin-releasing and insulin-like activity of Agaricus campestris (mushroom). J Endocrinol 157:259-266

Green TJ, Morhardt M, Brackett RG, Jacobs RL (1981) Serum inhibition of merozoite dispersal from Plasmodium falciparum schizonts: Indicator of immune status. Infect Immun 31:1203

Greeshma AA, Sridhar KR, Pavithra M, Tomita-Yokotani K (2018) Bioactive Potential of Nonconventional Edible Wild Mushroom Amanita. In: Gehlot P, Singh J (eds) Fungi and their Role in Sustainable Development: Current Perspectives. Springer, Berlin, pp 719-738

Gregori A, Pahor B, Glaser R, Pohleven F (2008) Influence of carbon dioxide, inoculum rate, amount and mixing of casing soil on Agaricus blazei fruiting bodies yield. Acta Agric Sloven 91:371378

Griesbach RJ (2002) Development of Phalaenopsis orchids for the mass-market. In: Janick J, Whipkey A (eds) Trends in new crops and new uses. ASHS Press, Alexandria

Grigera MS, Drijber RA, Wienhold BJ (2007) Increased abundance of arbuscular mycorrhizal fungi in soil coincides with the reproductive stages of maize. Soil Biol Biochem 39:1401-1409

Grimm D, Wösten HAB (2018) Mushroom cultivation in the circular economy. Appl Microbiol Biotechnol 102:7795-7803

Grishchenkov VG, Townsend RT, McDonald TJ, Autenrieth RL et al (2000) Degradation of petroleum hydrocarbons by facultative anaerobic bacteria under aerobic and anaerobic conditions. Process Biochem 35:889-896

Grothe T, Stadler M, Köpcke B, Roemer E et al (2011) Terpenoid spiro ketal compounds with LXR agonists activity, their use and formulations with them. World patent WO/2012/079721

Grove JF (1980) Cyclodepsipeptides from Beauveria bassiana. Part 3. The isolation of beauverolides $\mathrm{Ba}, \mathrm{Ca}$, Ja, and $\mathrm{Ka}$. J Chem Soc Perkin Trans 1:2878-2880

Grove JF, Pople M (1980) The insecticidal activity of beauvericin and the enniatin complex. Mycopathologia 70:103-105

Grove JF, MacMillan J, Mulholland TPC, Rogers MAT (1952) Griseofulvin. Part IV. Structure. J Chem Soc 3977-3987

Guerreiro MA, Brachmann A, Begerow D, Peršoh D (2018) Transient leaf endophytes are the most active fungi in 1-year-old beech leaf litter. Fungal Divers 89:237-251 
Guerrini L, Angeloni G, Baldi F, Parenti A (2017) Thermal effects of pump-overs during red wine fermentation. Appl Therm Eng 112:621-626

Guijarro B, Melgarejo P, Torres R, Lamarca N et al (2007) Effects of different biological formulations of Penicillium frequentans on brown rot of peaches. Biol Control 42:86-96

Guillamón E, García-Lafuente A, Lozano M, Rostagno MA, Villares A, Martínez JA (2010) Edible mushrooms: role in the prevention of cardiovascular diseases. Fitoterapia 81:715-723

Guo YX, Qin JZ, Chen JZ, Yang YC (2011) Study on the production of Agricus bisporus seasoning by enzyme method. J Shanxi Univ Sci Technol 29:53-57 In Chinese

Gupta S, Aggarwal S (2016) Dyeing wet blue goat nappa skin with a microbial colorant obtained from Penicillium minioluteum. J Clean Prod 127:585-590

Gupta C, Prakash D, Gupta S (2015) Biotechnological approach to microbial based perfumes and flavors. J Microbiol Exp 2:34-41

Gupte A, Tripathi A, Patel H, Rudakiya D, Gupte S (2016) Bioremediation of polycyclic aromatic hydrocarbon (PAHs): a perspective. Open Biotechnol J 10:363-378

Gupta S, Summuna B, Gupta M, Annepu SK (2018) Edible mushrooms: cultivation, bioactive molecules, and health benefits. In: Mérillon JM, Ramawat K (eds) Bioactive molecules in food. Springer, Berlin, pp 1-33

Gülser C, Pekşen A (2003) Using tea waste as a new casing material in mushroom Agaricus bisporus (L.) Sing. cultivation. Bioresour Technol 88:153-156

Günther M, Zibek S, Rupp S (2017) Fungal glycolipids as biosurfactants. Curr Biotechnol 6:205-218

Güvenç A, Kapucu N, Mehmetoğlu Ü (2002) The production of isoamyl acetate using immobilized lipases in a solvent-free system. Process Biochem 38:379-386

Haas BJ, Zeng Q, Pearson MD, Cuomo CA, Wortman JR (2011) Approaches to fungal genome annotation. Mycology 2:118-141

Hadibarata T, Kristanti RA (2013) Biodegradation and metabolite transformation of pyrene by basidiomycetes fungal isolate Armillaria sp. F022 36: 461. Bioprocess Biosyst Eng 36:461468

Hadibarata T, Teh ZC (2014) Optimization of pyrene degradation by white-rot fungus Pleurotus pulmonarius F043 and characterization of its metabolites. Bioprocess Biosyst Eng 37:1679-1684

Hagedorn S, Kaphammer B (1994) Microbial biocatalysis in the generation of flavor and fragrance chemicals. Annu Rev Microbiol 48:773-800

Haitjema CH, Gilmore SP, Henske JK, Solomon KV et al (2017) A parts list for fungal cellulosomes revealed by comparative genomics. Nat Microbiol 2:17087

Hall FR (1998) Biopesticides: use and delivery. Humana Press, Totowa, pp 233-270

Hamano PS, Kilikian BV (2006) Production of red pigments by Monascus ruber in culture media containing corn steep liquor. Braz J Chem Eng 23:443-449

Hameed I, Masoodi SR, Mir SA, Nabi M et al (2015) Type 2 diabetes mellitus: from a metabolic disorder to an inflammatory condition. World J Diabetes 6:598-612

Hameed A, Hussain SA, Yang J, Ijaz MU et al (2017) Antioxidants potential of the filamentous fungi (Mucor circinelloides). Nutrients 9:1101

Hamill RL, Higgens CE, Boaz HE, Gorman M (1969) The structure of beauvericin, a new depsipeptide antibiotic toxic to Artemia salina. Tetrahedron Lett 10:4255-4258

Han SY, Hong ZY, Xie YH, Zhao Y, Xu X (2017) Therapeutic effect of Chinese herbal medicines for post stroke recovery: a traditional and network meta-analysis. Medicine 96:1-13
Haneef M, Ceseracciu L, Canale C, Ilker B et al (2017) Advanced materials from fungal mycelium: fabrication and tuning of physical properties. Sci Rep 7:41292

Hansen MB, Jensen ML, Carstensen B (2012) Causes of death among diabetic patients in Denmark. Diabetologia 55:294-302

Hanson JR (2008) Fungal metabolites derived from amino acids. In: Hanson JR (ed) The chemistry of Fungi. The Royal Society of Chemistry, Cambridge, pp 32-46

Hao YJ, Zhao Q, Wang SX, Yang ZL (2016) What is the radicate Oudemansiella cultivated in China? Phytotaxa 286:1-12

Hapuarachchi KK, Wen TC, Jeewon R, Wu XL, Kang JC (2014) Mycosphere essays 7. Ganoderma lucidum-are the beneficial anti-cancer properties substantiated? Mycosphere 7:305-332

Hapuarachchi KK, Karunarathna SC, Phengsintham P, Kakumyan P, Hyde KD, Wen TC (2018) Amauroderma (Ganodermataceae, Polyporales)—bioactive compounds, beneficial properties and two new records from Laos. Asian J Mycol 1:121-136

Hapuarachchi KK, Karunarathna SC, McKenzie EHC, Wu XL, Kakumyan P, Hyde KD, Wen TC (2019) High phenotypic plasticity of Ganoderma sinense (Ganodermataceae, Polyporales) in China. Asian J Mycol 2:1-47

Haritash AK, Kaushik CP (2009) Biodegradation aspects of polycyclic aromatic hydrocarbons (PAHs): a review. J Hazard Mater 169:1-15

Hart MM, Trevors JT (2005) Microbe management: application of mycorrhizal fungi in sustainable agriculture. Front Ecol Environ 3:533-539

Hasan HA (2002) Gibberellin and auxin-indole production by plant root-fungi and their biosynthesis under salinity-calcium interaction. Acta Microbiol Immunol Hung 49:105-118

Hasan S, Ayres PG (1990) The control of weeds through fungi: principles and prospects. New Phytol 115:201-222

Hasan MT, Khatun MHA, Sajib MAM, Rahman MM et al (2015) Effect of wheat bran supplement with sugarcane bagasse on growth, yield and proximate composition of pink oyster mushroom Pleurotus djamor. Am J Food Sci Technol 3:150-157

Hawksworth DL (2012) Global species numbers of fungi: are tropical studies and molecular approaches contributing to a more robust estimate. Biodivers Conserv 21:2425-2433

Hawksworth DL, Lücking R (2017) Fungal diversity revisited: 2.2 to 3.8 million species. Microbiol Spectrum 5:1-17

He W, Zhao W, Yang R (2019) Effects of wheat gluten modified by deamidation-heating with three different acids on the microstructure of model oil-in-water emulsion and rheological-physical property of ice cream. Food Hydrocoll 87:679-690

Hecquet L, Sancelme M, Bolte J, Demuynck C (1996) Biosynthesis of 4-hydroxy-2,5-dimethyl-3(2H)-furanone by Zygosaccharomyces rouxii. J Agric Food Chem 44:1357-1360

Hedden P, Sponsel V (2015) A century of gibberellin research. J Plant Growth Regul 34:740-760

Heilmann-Clausen J, Barron ES, Boddy L, Dahlberg A et al (2014) A fungal perspective on conservation biology. Conserv Biol 29:6168

Helaly SE, Ashrafi S, Teponno RB, Bernecker S et al (2018a) Nematicidal cyclic lipodepsipeptides and a xanthocillin derivative from a phaeosphariaceous fungus parasitizing eggs of the plant parasitic nematode Heterodera filipjevi. J Nat Prod 81:2228-2234

Helaly SE, Thongbai B, Stadler M (2018b) Diversity of biologically active secondary metabolites from endophytic and saprotrophic fungi of the ascomycete order Xylariales. Nat Prod Rep 35:9921014

Heneghan MN, Yakasai A, Williams K, Kadir KA et al (2011) The programming role of trans-acting enoyl reductases during the biosynthesis of highly reduced fungal polyketides. Chem Sci 2:972-980 
Hernández VA, Galleguillos F, Thibaut R, Müller A (2018a) Fungal dyes for textile applications: testing of industrial conditions for wool fabric dyeing. J Textile Inst. https://doi.org/10.1080/ 00405000.2018.1460037

Hernández VA, Galleguillos FA, Sagredo N, Machuca Á (2018b) A note on the dyeing of wool fabrics using natural dyes extracted from rotten wood-inhabiting fungi. Coatings 8:77-83

Hernández-Montiel LG, Rueda-Puente EO, Cordoba-Matson MV, Holguín-Peña JR, Zulueta-Rodríguez R (2013) Mutualistic interaction of rizobacteria with arbuscular mycorrhizal fungi and its antagonistic effect on Fusarium oxysporum in Carica papaya seedlings. Crop Prot 47:61-66

Hesterkamp T (2017) Antibiotics clinical development and pipeline. In How to overcome the antibiotic crisis-facts, challenges, technologies \& future perspective (Stadler, M, Dersch P, eds.). Curr Top Microbiol Immunol 398:447-474

Hibbett DS, Vilgalys R (1993) Phylogenetic relationships of Lentinus (Basidiomycotina) inferred from molecular and morphological characters. Syst Bot 18:409-433

Hicks W (2011) Walter Hicks wine list 2011, p 92

Higgins C, Margot H, Warnquist S, Obeysekare E, Mehta K (2017) Mushroom cultivation in the developing world: a comparison of cultivation technologies. In: Global Humanitarian Technology Conference (GHTC), pp 1-7

Hill C, Guarner F, Reid G, Gibson GR et al (2014) The International Scientific Association for Probiotics and Prebiotics consensus statement on the scope and appropriate use of the term probiotic. Nat Rev Gastroenterol Hepatol 11:506-514

Himmel ME, Ding SY, Johnson DK, Adney WS et al (2007) Biomass recalcitrance: engineering plants and enzymes for biofuels production. Science 315:804-807

Hinsch E, Robinson S (2016) Mechanical colour reading of woodstaining fungal pigment textile dyes: an alternative method for determining colourfastness. Coatings 6:1-12

Hirotani M, Hirotani S, Takayanagi H, Yoshikawa T (1999) Blazeispirol A, an unprecedented skeleton from the cultured mycelia of the fungus Agaricus blazei. Tetrahedron Lett 40:329332

Hirotani M, Hirotani S, Yoshikawa T (2000) Blazeispirol X and Y, two novel carbon skeletal sterols from the cultured mycelia of the fungus Agaricus blazei. Tetrahedron Lett 41:5107-5110

Hirotani M, Hirotani S, Yoshikawa T (2001) Blazeispirol D and Z, as the actual intermediates of blazeispirol A biosynthesis from the cultured mycelia of the fungus Agaricus blazei. Tetrahedron Lett 42:5261-5264

Hirotani M, Sai K, Hirotani S, Yoshikawa T (2002) Blazeispirols B, $\mathrm{C}, \mathrm{E}$ and $\mathrm{F}$, des-A-ergostane-type compounds, from the cultured mycelia of the fungus Agaricus blazei. Phytochemistry 59:571577

Hoffmann C, Dollive S, Grunberg S, Chen J et al (2013) Archaea and fungi of the human gut microbiome: correlations with diet and bacterial residents. PLoS ONE 8:1-12

Hofmann T, Krautwurst D, Schieberle P (2018) Current status and future perspectives in flavor research: highlights of the 11th Wartburg Symposium on flavor chemistry \& biology. J Agric Food Chem 66:2197-2203

Hofrichter M, Lundell T, Hatakka A (2011) Conversion of milled pine wood by manganese peroxidase from Phlebia radiata. Appl Environ Microbiol 67:4588-4593

Holland RJ, Williams KL, Khan A (1999) Infection of Meloidogyne javanica by Paecilomyces lilacinus. Nematology 1:131-139

Hong SB, Kim DH, Samson RA (2015) Aspergillus associated with Meju, a fermented soybean starting material for traditional soy sauce and soybean paste in Korea. Mycobiology 43:218-224
Horbach R, Quesada ARN, Knogge W, Deising HB (2011) When and how to kill a plant cell: infection strategies of plant pathogenic fungi. J Plant Physiol 5:722-732

Hornsey IS (2003) A history of beer and brewing. The Royal Society of Chemistry, Cambridge

Hornsey IS (2012) Alcohol and its role in the evolution of human society. Royal Society of Chemistry, London

Horta MAC, Filho JAF, Murad NF, de Oliveira SE et al (2018) Network of proteins, enzymes and genes linked to biomass degradation shared by Trichoderma species. Sci Rep 8:1-11

Hosseini F, Mosaddeghi MR, Dexter AR (2017) Effect of the fungus Piriformospora indica on physiological characteristics and root morphology of wheat under combined drought and mechanical stresses. Plant Physiol Biochem 118:107-120

Hou XQ, Guo SX (2009) Interaction between a dark septate endophytic isolate from Dendrobium sp. and roots of $D$. nobile seedlings. J Integr Plant Biol 51:374-381

Hou CW, Jeng KC, Chen YS (2010) Enhancement of fermentation process in Pu-erh tea by tea-leaf extract. J Food Sci 75:44-48

Howard GT (2012) Polyurethane biodegradation. In: Singh SN (ed) Microbial degradation of xenobiotics. Springer, Berlin, pp 371394

Hua MDS, Kumar RS, Shyur LF, Cheng YB et al (2017) Metabolomic compounds identified in Piriformospora indicacolonized Chinese cabbage roots delineate symbiotic functions of the interaction. Sci Rep 7:1-14

Huang WY, Cai YZ, Surveswaran S, Hyde KD, Corke H, Sun M (2009) Molecular phylogenetic identification of endophytic fungi isolated from three Artemisia species. Fungal Divers 36:69-88

Huang L, Wei PL, Zang R, Xu ZN, Cen PL (2010) High-throughput screening of high-yield colonies of Rhizopus oryzae for enhanced production of fumaric acid. Ann Microbiol 60:287292

Huang R, Li GQ, Zhang J, Yang L et al (2011) Control of postharvest Botrytis fruit rot of strawberry by volatile organic compounds of Candida intermedia. Phytopathology 101:859-869

Huang Y, Kuang Z, Wang W, Cao L (2016) Exploring potential bacterial and fungal biocontrol agents transmitted from seeds to sprouts of wheat. Biol Control 98:27-33

Huang MY, Lin KH, Lu CC, Chen LR et al (2017a) The intensity of blue light-emitting diodes influences the antioxidant properties and sugar content of oyster mushrooms (Lentinus sajor-caju). Sci Hort 218:8-13

Huang Z, Nong X, Wang J, Zhang X et al (2017b) Anti-HSV-1, antioxidant and antifouling phenolic compounds from the deepsea-derived fungus Aspergillus versicolor SCSIO 41502. Bioorg Med Chem Lett 27:787-789

Huang H, Wang SL, Nguyen V, Kuo YH (2018) Isolation and identification of potent antidiabetic compounds from Antrodia cinnamomea - an edible Taiwanese mushroom. Molecules 23:112

Hulea A (1939) Contributions à la connaissance des champignons commensaux des Urédinées. Bulletin de la Section Scientifique de l'Académie de Roumanie 22:1-19

Huseyin CE, O'Toole PW, Cotter PD, Scanlan PD (2017) Forgotten fungi-the gut mycobiome in human health and disease. FEMS Microbiol Rev 41:479-511

Hussain A, Rizwan-ul-Haq M, Al-Ayedh H, Al-Jabr M (2014) Mycoinsecticides: potential and future perspective. Recent Pat Food Nutr Agric 6:45-53

Hutchings ML, Alpha-Cobb CJ, Hiller DA, Berro J, Strobel SA (2017) Mycofumigation through production of the volatile DNA methylating agent $\mathrm{N}$-methyl-N-nitrosoisobutyramide by fungi in the genus Muscodor. J Biol Chem 292:7358-7371

Hyde KD, Soytong K (2008) The fungal endophyte dilemma. Fungal Divers 33:163-173 
Hyde KD, Bahkali AH, Moslem MA (2010) Fungi-an unusual source for cosmetics. Fungal Divers 43:1-9

Hyde KD, Nilsson RH, Alias SA, Ariyawansa HA et al (2014) One stop shop: backbones trees for important pytopathogenic genera: I. Fungal Divers 67:21-125

Hyde KD, Hongsanan S, Jeewon R, Bhat DJ et al (2016) Fungal diversity notes 367-490: taxonomic and phylogenetic contributions to fungal taxa. Fungal Divers 80:1-270

Hyde KD, Norphanphoun C, Abreu VP, Bazzicalupo A et al (2017) Fungal diversity notes 603-708: taxonomic and phylogenetic notes on genera and species. Fungal Divers 87:1-235

Hyde KD, Al-Hatmi AM, Andersen B, Boekhout T, Buzina W, Dawson TL, Eastwood DC, Jones EG, de Hoog S, Kang Y, Longcore JE (2018a) The world's ten most feared fungi. Fungal Divers 93:161-194

Hyde KD, Chaiwan N, Norphanphoun C, Boonmee S et al (2018b) Mycosphere notes 169-224. Mycosphere 9:271-430

Hyde KD, Norphanphoun C, Chen J, Dissanayake AJ et al (2018c) Thailand's amazing diversity: up to $96 \%$ of fungi in northern Thailand may be novel. Fungal Divers 93:215-239

Hynninen PH, Räisänen R, Elovaara P, Nokelainen E (2000) Preparative isolation of anthraquinones from the fungus Dermocybe sanguinea using enzymatic hydrolysis by the endogenous ß-glucosidase. Z Naturforsch 55:600-610

Hyodo I, Amano N, Eguchi K, Narabayashi M et al (2005) Nationwide survey on complementary and alternative medicine in cancer patients in Japan. J Clin Oncol 23:2645-2654

Ibrahim AA (1994) Effect of cadusafos, Paecilomyces lilacinus and Nemout on reproduction and damage potential of Meloidogyne javanica. Pak J Nematol 12:141-147

Igiri BE, Okoduwa SIR, Idoko GO, Akabuogu EP et al (2018) Toxicity and bioremediation of heavy metals contaminated ecosystem from Tannery wastewater: a review. J Toxicol 2018:1-16

Ilmberger N, Meske D, Juergensen J, Schulte M et al (2012) Metagenomic cellulases highly tolerant towards the presence of ionic liquids-linking thermostability and halotolerance. Appl Microbiol Biotechnol 95:135-146

Imhoff JF, Labes A, Wiese J (2011) Bio-mining the microbial treasures of the ocean: new natural products. Biotechnol Adv 29:468-482

Ina K, Kataoka T, Ando T (2013) The use of lentinan for treating gastric cancer. Anticancer Agents Med Chem 13:681-688

Inglis DG, Enkerli J, Goettel M (2012) Laboratory techniques used for entomopathogenic fungi. Hypocreales. In: Lacey L (ed) Manual of techniques in invertebrate pathology, 2nd edn. Academic Press, Boston, pp 189-253

Interstate Technology and Regulatory Council (ITRC) (2002) A systematic approach to in situ bioremediation in ground water including decisions trees on in situ bioremediation for nitrates, carbon tetrachloride and perchlorate. https://clu-in.org/conf/itrc/ sysisb/. Accessed 3 Mar 2019

Islam MR, Tudryn G, Bucinell R, Schadler L, Picu C (2018) Mechanical behavior of mycelium-based particulate composites. J Mater Sci 53:16371-16382

Isogai A, Kanaoka M, Matsuda H, Hori Y, Suzuki A (1978) Structure of a new cyclodepsipeptide, Beauverilide A from Beauveria bassiana. Agric Biol Chem 42:1797-1798

Itelima JU, Bang WJ, Onyimba IA, Egbere OJ (2018) A review: biofertilizer - a key player in enhancing soil fertility and crop productivity. J Microbiol Biotechnol Rep 2:22-28

Jaber LR, Ownley BH (2018) Can we use entomopathogenic fungi as endophytes for dual biological control of insect pests and plant pathogens? Biol Control 116:36-45

Jackson AJ, Walters DR, Marshall G (1997) Antagonistic interactions between the foliar pathogen Botrytis fabae and isolates of
Penicillium brevicompactum and Cladosporium cladosporioides on faba beans. Biol Control 8:97-106

Jahromi MF, Liang JB, Ho YW, Mohamad R, Goh YM, Shokryazdan P (2012) Lovastatin production by Aspergillus terreus using agro-biomass as substrate in solid state fermentation. J Biomed Biotechnol 2012:1-11

Jaivel N, Marimuthu P (2010) Optimization of lovastatin production in solid state fermentation by Aspergillus terreus. Int J Eng Sci 2:2730-2733

Jalaluddin M (1977) A useful pathological condition of wood. Econ Bot 31:222-224

Janisiewicz WJ, Jeffers SN (1997) Efficacy of commercial formulation of two biofungicides for control of blue mold and gray mold of apples in cold storage. Crop Prot 16:629-633

Janoušková M, Krak K, Vosátka M, Püschel D, Storchová H (2017) Inoculation effects on root-colonizing arbuscular mycorrhizal fungal communities spread beyond directly inoculated plants. PLoS ONE 12:e181525

Jansson HB, Jeyaprakash A, Zuckerman BM (1985) Control of rootknot nematodes on tomato by endoparasitic fungus Meria coniospora. J Nematol 17:327-329

Jansson HB, Lopez-Llorca LV (2004) Control of nematodes by fungi. In: Arora DK (ed) Fungal biotechnology in agricultural, food, and environmental applications, mycology series, vol 21. CRC Press, Boca Raton, pp 205-215

Jaronski ST, Mascarin GM (2017) Mass production of fungal entomopathogens. In: Lacey LA (ed) Microbial control of insects and mite pests, vol 9. Academic Press, Amsterdam, pp 141-155

Jaspers NG, Raams A, Kelner MJ, Ng JM et al (2002) Anti-tumour compounds illudin $\mathrm{S}$ and Irofulven induce DNA lesions ignored by global repair and exclusively processed by transcription-and replication-coupled repair pathways. DNA Repair 1:1027-1038

Jayawardena RS, Hyde KD, Damm U, Cai L et al (2016) Notes on currently accepted species of Colletotrichum. Mycosphere 7:1192-1260

Jenkins MJ, Harrison KL (2008) The effect of crystalline morphology on the degradation of polycaprolactone in a solution of phosphate buffer and lipase. Polym Adv Technol 19:1901-1906

Jeon S, Bose S, Hur J, Jun K, Kim YK, Cho KS, Koo BS (2011) A modified formulation of Chinese traditional medicine improves memory impairment and reduces $A \beta$ level in the Tg-APPswe/ PS1dE9 mouse model of Alzheimer's disease. J Ethnopharmacol 137:783-789

Jeong SC, Jeong YT, Yang BK, Islam R et al (2010) White button mushroom (Agaricus bisporus) lowers blood glucose and cholesterol levels in diabetic and hypercholesterolemic rats. Nutr Res 30:49-56

Jeschke R, Iinuma K, Harder A, Schindler M, Murakami T (2005) Influence of the cyclooctadepsipeptides PF1022A and PF1022E as natural products on the design of semi-synthetic anthelmintics such as emodepside. Parasitol Res 97(1):S11-S16

Jeznabadi EK, Jafarpour M, Eghbalsaied S (2016) King oyster mushroom production using various sources of agricultural wastes in Iran. Int J Recycl Org Waste Agric 5:17-24

Jha BN, Sharma GD, Shukla AK (2008) Effect of ectomycorrhizal development on growth in pine seedling. J Plant Sci 3:77-84

Ji KP, Cao Y, Zhang CX, He MX et al (2011) Cultivation of Phlebopus portentosus in southern China. Mycol Pro 10:293300

Jiang YF, Chang TJ, Liu Y, Zhang M et al (2017) Overview of Ganoderma sinense polysaccharide-an adjunctive drug used during concurrent chemo/radiation therapy for cancer treatment in China. Biomed Pharmacother 96:865-870

Jiménez DJ, Chaib De Mares M, Salles JF (2018) Temporal expression dynamics of plant biomass-degrading enzymes by a 
synthetic bacterial consortium growing on sugarcane bagasse. Front Microbiol 9:299

Jin X, Custis D (2011) Microencapsulating aerial conidia of Trichoderma harzianum through spray drying at elevated temperatures. Biol Control 56:202-208

Jin Y, Qin S, Gao H, Zhu G et al (2018) An anti-HBV anthraquinone from aciduric fungus Penicillium sp. OUCMDZ-4736 under low pH stress. Extremophiles 22:39-45

Jogaiah S, Abdelrahman M, Tran LSP, Ito SI (2018) Different mechanisms of Trichoderma virens-mediated resistance in tomato against Fusarium wilt involve the jasmonic and salicylic acid pathways. Mol Plant Pathol 19:870-882

Jogawat A, Vadassery J, Verma N, Oelmüller R et al (2016) PiHOG1, a stress regulator MAP kinase from the root endophyte fungus Piriformospora indica, confers salinity stress tolerance in rice plants. Sci Rep 6:36765

Johjima T, Itoh N, Kabuto M, Tokimura F et al (1999) Direct interaction of lignin and lignin peroxidase from Phanerochaete chrysosporium. PNAS 96:1989-1994

Jones N (2013) Food fuelled with fungi. Nature 504:199

Jones M, Bhat T, Wang C, Moinuddin K, John S (2017) Thermal degradation and fire properties of fungal mycelium and mycelium- biomass composite materials. Sci Rep 8:1-10

Jones M, Bhat T, Huynh T, Kandare E et al (2018) Waste-derived low-cost mycelium composite construction materials with improved fire safety. Fire Mater 9:71-80

Julien MH, Griffiths MW (1998) Biological control of weeds, a world catalogue of agents and their target weeds, 4th edn. CAB International, Wallingford, p p223

Jung JH, Lee HW, Lee YJ, Lee HR, Um TH, Park JY, Yi SY (2015) Four cases of acquired aplastic anemia affecting an entire family after ingesting Ganodermalucidum. Korean J Medicine 88:94100

Justo A, Hibbett DS (2011) Phylogenetic classification of Trametes (Basidiomycota, Polyporales) based on a five-marker dataset. Taxon 60:1567-1583

Kabaluk JT, Svircev AM, Goettel MS, Woo SG (eds) (2010) The use and regulation of microbial pesticides in representative jurisdictions worldwide. IOBC Global, St. Paul

Kabir Y, Kimura S (1989) Dietary mushrooms reduce blood preassure in spontaneously hypertensive rats (SHR). J Nutr Sci Vitaminol 35:91-94

Kabir Y, Kimura S, Tamura T (1988) Dietary effect of Ganoderma lucidum mushroom on blood pressure and lipid levels in spontaneously hypertensive rats (SHR). J Nutr Sci Vitaminol 34:433-438

Kaewchai S, Soytong K, Hyde KD (2009) Mycofungicides and fungal biofertilizers. Fungal Divers 38:25-50

Kaewgrajang T, Sangwanit U, Kodama M, Yamota M (2013) Ectomycorrhizal fungal communities of Dipterocarpus alatus seedlings introduced by soil inocula from a natural forest and a plantation. J For Res 9:260-267

Kalač P (2013) A review of chemical composition and nutritional value of wild-growing and cultivated mushrooms. J Sci Food Agric 93:209-218

Kalra EK (2003) Nutraceutical-definition and introduction. AAPS Pharm Sci 5:27-28

Kamei I, Takagi K, Kondo R (2011) Degradation of endosulfan and endosulfan sulfate by white-rot fungus Trametes hirsuta. J Wood Sci $57: 317-322$

Kamthan R, Tiwari I (2017) Agricultural wastes - Potential substrates for mushroom cultivation. Eur Exp Biol 7:31

Kaneko M, Watashi K, Kamisuki S, Matsunaga H et al (2015) A novel tricyclic polyketide, vanitaracin A, specifically inhibits the entry of hepatitis B and D viruses by targeting sodium taurocholate co-transporting polypeptide. J Virol 89:1194511953

Kaplan GG (2015) The global burden of IBD: from 2015 to 2025. Nat Rev Gastroenterol Hepatol 12:720-727

Kappler A, Wuestner ML, Ruecker A, Harter J et al (2014) Biochar as an electron shuttle between bacteria and $\mathrm{Fe}(\mathrm{III})$ minerals. Environ Sci Technol Lett 1:339-344

Karwehl S, Stadler M (2017) Exploitation of fungal biodiversity for discovery of novel antibiotics the antibiotic crisis-facts, challenges, technologies \& future perspective. Curr Top Microbiol Immunol 398:303-338

Kathiresan K, Manivannan S (2006) $\alpha$-Amylase production by Penicillium fellutanum isolated from mangrove rhizosphere soil. Afr J Biotechnol 5:829-832

Kaur G, Kalia A, Sodhi HS (2018) Selenium biofortification of Pleurotus species and its effect on yield, phytochemical profiles, and protein chemistry of fruiting bodies. J Food Biochem 42:12467

Kayama M, Yamanaka T (2016) Growth characteristics of ectomycorrhizal seedlings of Quercus glauca, Quercus salicina, Quercus myrsinaefolia, and Castanopsis cuspidate planted in calcareous soil. Forests 7:266

Kazan K, Lyons R (2014) Intervention of phytohormone pathways by pathogen effectors. Plant Cell 26:2285-2309

Keener PD (1954) Cladosporium aecidiicola Thuem. and Tubercolina persicina (Ditm). Sacc. associated with Puccinia conspicua (Arth.) Mains on Helenium hoopesii A. Gray in Arizona. Plant Dis Rep 38:691-694

Kefialew Y, Ayalew A (2008) Postharvest biological control of anthracnose (Colletotrichum gloeosporioides) on mango (Mangifera indica). Postharvest Biol Technol 50:8-11

Kendrick B (1992) The fifth kingdom. Hackett Publishing, Indianapolis

Kent JM (1994) Plant protection B-notes and readings: weeds. Charles Sturt University, Sydney Olympic Park

Keppanan R, Sivaperumal S, Ramos Aguila LC, Hussain M et al (2018) Isolation and characterization of Metarhizium anisopliae TK29 and its mycoinsecticide effects against subterranean termite Coptotermes formosanus. Microb Pathog 123:52-59

Kerrigan RW (2005) Agaricus subrufescens, a cultivated edible and medicinal mushroom, and its synonyms. Mycologia 97:12-24

Kersey PJ, Lawson D, Birney E, Derwent PS et al (2010) Ensembl genomes: extending Ensembl across the taxonomic space. Nucleic Acids Res 38:D563-D569

Kershaw MJ, Moorhouse ER, Bateman R, Reynolds SE, Charnley AK (1999) The role of destruxins in the pathogenicity of Metarhizium anisopliae for three species of insect. J Invert Pathol 74:213-223

Keshavarz B, Khalesi M (2016) Trichoderma reesei, a superior cellulase source for industrial applications. Biofuels 7:713-721

Khan AL, Hamayun M, Kang SM, Kim YH et al (2012) Endophytic fungal association via gibberellins and indole acetic acid can improve plant growth under abiotic stress: an example of Paecilomyces formosus LHL10. BMC Microbiol 12:1-14

Khan AL, Hussain J, Al-Harrasi A, Al-Rawahi A, Lee IJ (2015a) Endophytic fungi: resource for gibberellins and crop abiotic stress resistance. Crit Rev Biotechnol 35:62-74

Khan AR, Ullah I, Waqas M, Shahzad R et al (2015b) Plant growthpromoting potential of endophytic fungi isolated from Solanum nigrum leaves. World J Microbiol Biotechnol 31:1461-1466

Khan S, Nadir S, Shah ZU, Shah AA et al (2017) Biodegradation of polyester polyurethane by Aspergillus tubingensis. Environ Pollut 225:469-480

Kiho T, Yamane A, Hui J, Usui S, Ukai S (1996) Polysaccharides in fungi. XXXVI Hypoglycemic activity of a polysaccharide (CSF30) from the cultural mycelium of Cordyceps sinensis and its 
effect on glucosemetabolism in mouse liver. Biol Pharm Bull 19:294-296

Kildgaard S, Mansson M, Dosen I, Klitgaard A et al (2014) Accurate dereplication of bioactive secondary metabolites from marinederived fungi by UHPLC-DAD-QTOFMS and a MS/HRMS library. Mar Drugs 12:3681-3705

Kim AY (2005) Application of biotechnology to the production of natural flavor and fragrance chemicals. In: ACS symposium series. Oxford University Press, pp 60-75

Kim JD (2007) Purification and characterization of a keratinase from a feather-degrading fungus, Aspergillus flavus Strain K-03. Mycobiology 35:219-225

Kim DY, Rhee YH (2003) Biodegradation of microbial and synthetic polyesters by fungi. Appl Microbiol Biotechnol 61:300-308

Kim S, Dale BE (2004) Global potential bioethanol production from wasted crops and crop residues. Biomass Bioenerg 26:361-375

Kim KH, Kim JY, Cho TS, Choi JW (2012) Influence of pyrolysis temperature on physicochemical properties of biochar obtained from the fast pyrolysis of pitch pine (Pinus rigida). Bioresour Technol 118:158-162

Kim YW, Kim KH, Choi HJ, Lee DS (2005) Anti-diabetic activity of $\beta$-glucans and their enzymatically hydrolyzed oligosaccharides from Agaricus blazei. Biotechnol Lett 27:483-487

Kinsella JE, Hwang DH, Dwivedi B (1976) Enzymes of Penicillium roqueforti involved in the biosynthesis of cheese flavor. Crit Rev Food Sci Nutr 8:191-228

Kipfer T, Wohlgemuth T, van der Heiden MGA, Ghazoul J, Egli S (2012) Growth response of drought-stressed Pinus sylvestris seedlings to single- and multi-species inoculation with ectomycorrhizal fungi. PLoS ONE 7:e35275

Kirk O, Borchert TV, Fuglsang CC (2002) Industrial enzyme applications. Curr Opin Biotechnol 13:345-351

Kirk MD, Pires SM, Black RE, Caipo M (2015) World Health Organization estimates of the global and regional disease burden of 22 foodborne bacterial, protozoal, and viral diseases, 2010: a data synthesis. PLoS Med 12(12):e1001921

Klinsupa W, Phansiri S, Thongpradis P, Yongsmith B, Pothiratana C (2016) Enhancement of yellow pigment production by intraspecific protoplast fusion of Monascus spp. yellow mutant (ade-) and white mutant (prototroph). J Biotechnol 217:62-71

Kloareg B, Quatrano RS (1988) Structure of the cell walls of marine algae and ecophysiological functions of the matrix polysaccharides. Oceanogr Mar Biol Annu Rev 26:259-315

Klomklung N, Karunarathna SC, Chukeatirote E, Hyde KD (2012) Domestication of wild strain of Pleurotus giganteus. Sydowia 60:39-53

Klüpfel L, Keiluweit M, Kleber M, Sander M (2014) Redox properties of plant biomass-derived black carbon (Biochar). Environ Sci Technol 48:5601-5611

Knight DW, Pattenden G (1976) Synthesis of the pulvinic acid pigments of lichen and fungi. J Chem Soc Chem Com 639:660 661

Ko HG, Park SH, Kim SH, Park HG, Park WM (2005) Detection and recovery of hydrolytic enzymes from spent compost of four mushroom species. Folia Microbiol 50:103-106

Konno S, Alexander B, Zade J, Choudhury M (2013) Possible hypoglycemic action of SX-fraction targeting insulin signal transduction pathway. Int J Gen Med 6:181

Kozarski M, Klaus A, Niksic M, Jakovljevic D et al (2011) Antioxidative and immunomodulating activities of polysaccharide extracts of the medicinal mushrooms Agaricus bisporus, Agaricus brasiliensis, Ganoderma lucidum, and Phellinus linteus. Food Chem 129:1667-1675

Kozubal MA, Macur RE, Avniel YC, Sustainable Bioproducts, Inc. (2019) Filamentous fungal biomats, methods of their production and methods of their use. US patent application 16/118:370
Kranz J (1981) Hyperparasitism of biotrophic fungi. In: Blakeman JP (ed) Microbial ecology of the phylloplane. Academic Press, London, pp 327-352

Krasowska K, Janik H, Gradys A, Rutkowska M (2012) Degradation of polyurethanes in compost under natural conditions. J Appl Polym Sci 125:4252-4260

Krings U, Berger RG (1998) Biotechnological production of flavors and fragrances. Appl Microbiol Biotechnol 49:1-8

Kristiansen KA, Taylor DL, Kjoller R, Rasmussen HN, Rosendahl S (2001) Identification of mycorrhizal fungi from single pelotons of Dactylorhiza majalis (Orchidaceae) using single-strand conformation polymorphism and mitochondrial ribosomal large subunit DNA sequences. Mol Ecol 10(8):2089-2093

Kristiansen KA, Freudenstein JV, Rasmussen FN, Rasmussen HN (2004) Molecular identification of mycorrhizal fungi in Neuwiedia veratrifolia (Orchidaceae). Mol Phylogenet Evol 33:251-258

Krištofíková L, Rosenberg M, Vlnova A, Šajbidor J, Čertík M (1991) Selection of Rhizopus strains for 1 (+)-lactic acid and $\gamma$-linolenic acid production. Folia Microbiol 36:451-455

Krogerus K, Magalhães F, Vidgren V, Gibson B (2017) Novel brewing yeast hybrids: creation and application. Appl Microbiol Biotechnol 101:65-78

Krügener S, Krings U, Zorn H, Berger RG (2010) A dioxygenase of Pleurotus sapidus transforms (+)-valencene regio-specifically to (+)-nootkatone via a stereo-specific allylic hydroperoxidation. Bioresour Technol 101:457-462

Krüger D, Kreisel H (2003) Proposing Morganella subgen. Apioperdon subgen. nov. for the puffball Lycoperdon pyriforme. Mycotaxon 86:169-177

Kuanyshev N, Adamo GM, Porro D, Branduardi P (2017) The spoilage yeast Zygosaccharomyces bailii: Foe or friend? Yeast 34:359-370

Kubo K, Namba H (1997) Anti-hyperliposis effect of Maitake fruit body (Grifola frondosa). Biol Pharm Bull 20:781-785

Kubo T, Terabayashi S, Takeda S, Sasaki H et al (2006) Indoor cultivation and cultural characteristics of Wolfiporia cocos sclerotia using mushroom culture bottles. Biol Pharm Bull 29:1191-1196

Kudalkar P, Strobel G, Hassan S, Geary B, Sears J (2012) Muscodor sutura, a novel endophytic fungus with volatile antibiotic activities. Mycoscience 53:319-325

Kuhnert E, Li Y, Lan N, Yue Q et al (2018) Enfumafungin synthase represents a novel lineage of fungal triterpene cyclases. Environm Microbiol 1:12. https://doi.org/10.1111/1462-2920.14333

Kulshreshtha S, Mathur N, Bhatnagar P (2014) Mushroom as a product and their role in mycoremediation. AMB Express 4:29

Kumar R, Kaushik P (2004) Isolation of a cellulose producing mycorrhizal fungus Rhizoctonia solani from Zeuxine strateumatica (Linn.) Schltr. J Orchid Soc India 18:11108

Kumar U, Berliner J, Adak T, Rath PC et al (2017) Non-target effect of continuous application of chlorpyrifos on soil microbes, nematodes and its persistence under sub-humid tropical rice-rice cropping system. Ecotoxicol Environ Saf 135:225-235

Kumla J, Danell E, Bussaban B, Lumyong S (2011) Suitable growth conditions and nutrition factors on in vitro culture of Phlebopus portentosus (Boletales). Chiang Mai J Sci 38:156-159

Kumla J, Danell E, Lumyong S (2015) Improvement of yield for a tropical black bolete, Phlebopus portentosus, cultivation in northern Thailand. Mycoscience 56:114-117

Kumla J, Suwannarach N, Lumyong S (2016) Characterization of Pisolithus orientalis in culture and in vitro mycorrhization with Eucalyptus camaldulensis and Pinus kesiya. Mycosphere 7:1415-1424

Kumura H, Tanoue Y, Tsukahara M, Tanaka T et al (2004) Screening of dairy yeast strains for probiotic applications. J Dairy Sci 87:4050-4056 
Kupcová K, Stefanova I, Plavcova Z, Hosek J et al (2018) Antimicrobial, cytotoxic, anti-Inflammatory, and antioxidant activity of culinary processed shiitake medicinal mushroom (Lentinus edodes, Agaricomycetes) and its major sulfur sensoryactive compound-Lenthionine. Int J Med Mushrooms 20:165175

Kurtzman CP, Fell JW, Boekhout T (2011) The yeasts, a taxonomic study, 5th edn. Elsevier, Burlington

Kwon H, Thatithatgoon S (2004) Mushroom growing for a living worldwide: mushroom growing in Northern Thailand. In: Gush $\mathrm{R}$ (ed) Mushroom growers' handbook 1: oyster mushroom cultivation. Mush World-Heineart Inc, Seoul

Kües U (2015) Fungal enzymes for environmental management. Curr Opin Biotechnol 33:268-278

Lacey LA, Kaya HK (2007) Field manual of techniques in invertebrate pathology. Application and evaluation of pathogens for control of insects and other invertebrate pests, 2nd edn. Springer, Dordrecht, $\mathrm{p} 484$

Lacey LA, Neven LG (2006) The potential of the fungus, Muscodor albus, as a microbial control agent of potato tuber moth (Lepidoptera: Gelechiidae) in stored potatoes. J Invertebr Pathol 91:195-198

Lacey LA, Grzywacz D, Shapiro-Ilan DI, Frutos R, Brownbridge M, Goettel MS (2015) Insect pathogens as biological control agents: back to the future. J Invert Pathol 132:1-41

Lai LST, Pan CC, Tzeng BK (2003) The influence of medium design on lovastatin production and pellet formation with a highproducing mutant of Aspergillus terreus in submerged cultures. Process Biochem 38:1317-1326

Lal RJ, Sinha OK, Bhatnagar S, Lal S, Awasthi SK (2009) Biological control of sugarcane smut (Sporisorium scitamineum) through botanicals and Trichoderma viride. Sugar Tech 11:381-386

Lale G, Jogdand VV, Gadre RV (2006) Morphological mutants of Gibberella fujikuroi for enhanced production of gibberellic acid. J Appl Microbiol 100:65-72

Lamar RT, White RB (2001) Mycoremediation: commercial status and recent developments. In: Magar VS et al (eds) Proceedings of the sixth international symposium on in situ and on-site bioremediation, San Diego, CA, vol 6, pp 263-278

Lambert C, Wendt L, Hladki AI, Stadler M, Sir EB (2019) Hypomontagnella (Hypoxylaceae): a new genus segregated from Hypoxylon by a polyphasic taxonomic approach. Mycol Prog 18:187-201

Lang E, E’Uer G, Kleeberg I, Martens R, Zadrazil F (1995) Interaction of white rot fungi and soil microorganisms leading to biodegradation of soil pollutants. In: Van Den Brink WJ, Bosman R, Arendt F (eds) Contaminated soil '95. Soil \& environment, vol 5. Springer, Dordrecht

Lange L, Pilgaard B, Herbest F-A, Busk PK, Gleason F, Pedersen AG (2018) Origin of fungal biomass degrading enzymes: evolution, diversity and function of enzymes of early lineage fungi. Fungal Biol Rev 33:82-97

Largent DL, Bradshaw DE, Wood WF (1990) The candy-like odor of Nolanea fructufragrans. Mycologia 82:786-787

Larsson E, Jeppson M (2008) Phylogenetic relationships among species and genera of Lycoperdaceae based on ITS and LSU sequence data from North European taxa. Mycol Res 112:4-22

Lassois L, de Bellaire LDL, Jijakli MH (2008) Biological control of crown rot of bananas with Pichia anomala strain K and Candida oleophila strain O. Biol Control 45:410-418

Laurie JD, Ali S, Linning R, Mannhaupt G et al (2012) Genome comparison of barley and maize smut fungi reveals targeted loss of RNA silencing components and species-specific presence of transposable elements. Plant Cell 24:1733-1745

Le Bloc'h J, Pauquai T, Bourges C (2015) Authorised EU health claim for red yeast rice. In: Sadler M (ed) Foods, nutrients and food ingredients with authorised EU health claims, vol 2. Elsevier, Amsterdam, pp 139-142

Le Jeune C, Lollier M, Demuyter C, Erny C et al (2007) Characterization of natural hybrids of Saccharomyces cerevisiae and Saccharomyces bayanus var. uvarum. FEMS Yeast Res $7: 540-549$

Leake JR (2004) Myco-heterotroph/epiparasitic plant interactions with ectomycorrhizal and arbuscular mycorrhizal fungi. Curr Opin Plant Biol 7:422-428

Lecomte C, Alabouvette C, Edel-Hermann V, Robert F, Steinberg C (2016) Biological control of ornamental plant diseases caused by Fusarium oxysporum: a review. Biol Control 101:17-30

Lee G, Arepally GM (2012) Anticoagulation techniques in apheresis: from heparin to citrate and beyond. J Clin Apheresis 27:117-125

Lee SY, Mohamed R (2016) The origin and domestication of Aquilaria, an important agarwood-producing genus. In: Mohamed R (ed) Agarwood. Springer, Singapore, pp 1-20

Lee IK, Yun BS (2006) Hispidin analogs from the mushroom Inonotus xeranticus and their free radical scavenging activity. Bioorg Med Chem Lett 16:2376-2379

Lee KH, Wi SG, Singh AP, Kim YS (2004) Micromorphological characteristics of decayed wood and laccase produced by the brown-rot fungus Coniophora puteana. J Wood Sci 50:281-284

Lee JS, Baik HS, Park SS (2006) Purification and characterization of two novel fibrinolytic proteases from mushroom Fomitella fraxinea. J Microbiol Biotechnol 16:264-271

Lee SS, Patahayah M, Chong WS, Lapeyrie F (2008a) Successful ectomycorrhizal inoculation of two dipterocarp species with a locally isolated fungus in Peninsular Malaysia. J Trop For Sci 20:237-247

Lee SW, Lee EH, Eom AH (2008b) Effects of organic farming on communities of arbuscular mycorrhizal fungi. Mycobiology 36:19-23

Lee SO, Kim HY, Choi GJ, Lee HB et al (2009a) Mycofumigation with Oxyporus latemarginatus EF069 for control of postharvest apple decay and Rhizoctonia root rot on moth orchid. J Appl Microbiol 106:1213-1219

Lee SS, Chang YS, Noraswati MNR (2009b) Utilization of macrofungi by some indigenous communities for food and medicine in Peninsular Malaysia. For Ecol Manag 257:2062-2065

Lee KH, Morris-Natschke SL, Yang X, Huang R et al (2012) Recent progress of research on medicinal mushrooms, foods, and other herbal products used in traditional Chinese medicine. J Trad Complement Med 2:1-12

Lehmann J, Joseph S (2009) Biochar for environmental management: an introduction. In: Lehmann J, Joseph $\mathrm{S}$ (eds) Biochar for environmental management: science and technology. Earthscan, London, pp 1-12

Leland JE, Mullins DE, Vaughan LJ, Warren HL (2005) Effects of media composition on submerged culture spores of the entomopathogenic fungus, Metarhizium anisopliae var. acridum Part 2: Effects of media osmolality on cell wall characteristics, carbohydrate concentrations, drying stability, and pathogenicity. Biocontrol Sci Technol 15:379-392

Lembeck H (1983) Grossman's guide to wines, beers, \& spirits, 7th edn. Charles Scribner's Sons, New York

Leong HY, Show PL, Lim MH, Ooi CW, Ling TC (2018) Natural red pigments from plants and their health benefits: a review. Food Rev Int 34:463-482

Lesage-Meessen L, Delattre M, Haon M, Thibault JF et al (1996) A two-step bioconversion process for vanillin production from ferulic acid combining Aspergillus niger and Pycnoporus cinnabarinus. J Biotechnol 50:107-113

Lesage-Meessen L, Bou M, Ginies C, Chevret D, Navarro D et al (2018) Lavender- and lavandin-distilled straws: an untapped feedstock with great potential for the production of high-added 
value compounds and fungal enzymes. Biotechnol Biofuels 11:217-229

Leskošek-Čukalović I, Despotovic S, Lakic L, Niksic M et al (2010) Ganoderma lucidum - medical mushroom as a raw material for beer with enhanced functional properties. Food Res Int 43:22622269

León-Santiesteban HH, Wrobel K, Revah S, Tomasini A (2016) Pentachlorophenol removal by Rhizopus oryzae CDBB-H-1877 using sorption and degradation mechanisms. J Chem Technol Biotechnol 91:65-71

Li C, Oberlies NH (2005) The most widely recognized mushroom: chemistry of the genus Amanita. Life Sci 78:532-538

Li SP, Zhao KJ, Ji ZN, Song ZH et al (2003) A polysaccharide isolated from cordyceps sinensis, a traditional Chinese medicine, protects PC12 cells against hydrogen peroxide-induced injury. Life Sci 73:2503-2513

Li WL, Zheng HC, Bukuru J, De Kimpe N (2004) Natural medicines used in the traditional Chinese medical system for therapy of diabetes mellitus. J Ethnopharmacol 92:1-21

Li Y, Hyde KD, Jeewon R, Cai L, Vijaykrishna D, Zhang KQ (2005) Phylogenetics and evolution of nematode-trapping fungi (Orbiliales) estimated from nuclear and protein coding genes. Mycologia 97:1034-1046

Li SP, Zhang GH, Zeng Q, Huang ZG, Wang YT, Dong TTX, Tsim KWK (2006) Hypoglycemic activity of polysaccharide, with antioxidation, isolated from cultured Cordyceps mycelia. Phytomedicine 13:428-433

Li G, Zhang K, Xu J, Dong J, Liu Y (2007) Nematicidal substances from fungi. Recent Pat Biotechnol 1:212-233

Li Y, Wang CL, Guo SX, Wang YJ et al (2009) Three new bibenzyl derivatives from Dendrobium candidum. Chem Pharm Bull 57:218-219

Li M, Hu J (2014) Study on survival strategies of farmers engage in small-scale household cultivation of edible mushrooms: take Shandong Province as an example. Mod Econ 5:1092-1100

Li GJ, Hyde KD, Zhao RN, Hongsanan S et al (2016) Fungal divers notes 253-366: taxonomic and phylogenetic contributions to fungal taxa. Fungal Divers 78:1-237

Li H, Yelle DJ, Li C, Yang $M$ et al (2017) Lignocellulose pretreatment in a fungus-cultivating termite. PNAS 114:47094714

Li C, Gao S, Yang X, Lin CSK (2018) Green and sustainable succinic acid production from crude glycerol by engineered Yarrowia lipolytica via agricultural residue based in situ fibrous bed bioreactor. Bioresour Technol 249:612-619

Li W, Yang Y, Dong W, Wang H et al (2019) Dimeric 2-(2phenylethyl)chromones from the agarwood of Aquilaria crassna in Laos. Fitoterapia 133:12-16

Liang B, Lehmann J, Solomon D, Kinyangi J et al (2006) Black carbon increases cation exchange capacity in soils. Soil Sci Soc Am J 70:1719-1730

Liang X, Csetenyi L, Gadd GM (2016) Lead bioprecipitation by yeasts utilizing organic phosphorus substrates. Geomicrobiol J 33:294-307

Liau CH, Lu JC, Prasad V, Hsiao HH et al (2003) The sweet pepper ferredoxin-like protein (pflp) conferred resistance against soft rot disease in Oncidium orchid. Transgenic Res 12:329-336

Lin HC, Hewage RT, Lu YC, Chooi YH (2019) Biosynthesis of bioactive natural products from Basidiomycota. Org Biomol Chem 7:1027-1036

Lin Y, Jia Y, Ke J, Sanders AJ et al (2015) Traditional Chinese medicine in the prevention and treatment of cancer and cancer metastasis (review). Oncol Lett 10:1240-1250

Lin F, Mei WL, Wu J, Dai HF (2010) GC-MS analysis of volatile constituents from Chinese eaglewood produced by artificial methods. Zhong Yao Cai 33:222-225
Ling LB, Ng TK (1989) Fermentation process for carboxylic acids. US patent 4,877,731, 31 Oct 1989

Lipczynska-Kochany E (2018) Humic substances, their microbial interactions and effects on biological transformations of organic pollutants in water and soil: a review. Chemosphere 202:420 437

Littlefield LJ (1981) Biology of the plant rusts: an introduction. Iowa State University Press, Iowa

Liu QJ (2009) Pu-erh tea appreciation and brewing. China Light Industry Press, Beijing

Liu S (2017) Soy Sauce Fermentation. In Soy Sauce Fermentation. In: Ray RC, Montet D (eds) Fermented foods, Part II: Technological interventions. CRC Press, Boca Raton, pp 135-149

Liu RJ, Chen YL (2007) Mycorrhizology. Science Press, Beijing

Liu XZ, Li S (2004) Fungal secondary metabolites in biological control of crop pests. In: An ZQ (ed) Handbook of industrial mycology. Marcel Dekker, New York, pp 723-747

Liu QJ, Zhou CQ, Xu HL et al (1986) The fermentation of Pu-erh tea. J Tea Sci 6:55-56

Liu X, Yuan CC, Chen XJ (2002) Anti-tumor activity of the sporoderm broken germinating spores of Ganoderma lucidum. Cancer Lett 182:155-161

Liu L, Agren R, Bordel S, Nielsen J (2010a) Use of genome-scale metabolic models for understanding microbial physiology. FEBS Lett 584:2556-2564

Liu L, Gao H, Chen X, Cai X et al (2010b) Brasilamides A-D: sesquiterpenoids from the plant endophytic fungus Paraconiothyrium brasiliense. Eur J Org Chem 17:3302-3306

Liu L, Niu S, Lu X, Chen X et al (2010c) Unique metabolites of Pestalotiopsis fici suggest a biosynthetic hypothesis involving a Diels-Alder reaction and then a mechanistic diversification. Chem Commun 46:460-462

Liu H, Liu S, Guo L, Zhang Y et al (2012a) New furanones from the plant endophytic fungus Pestalotiopsis besseyi. Molecules 17:14015-14021

Liu RM, Li YB, Zhong JJ (2012b) Cytotoxic and pro-apoptotic effects of novel ganoderic acid derivatives on human cervical cancer cells in vitro. Eur J Pharmacol 681:23-33

Liu W, Zhang M, Chang JH (2012c) Technology research of Bazhen mushroom soup. Food Sci Biotechnol 31:831-835

Liu YJ, Liu J, Ying SH, Liu SS, Feng MG (2013) A fungal insecticide engineered for fast per os killing of caterpillars has high field efficacy and safety in full-season control of cabbage insect pests. Appl Environ Microbiol 79:6452-6458

Liu JK, Hyde KD, Jones EBG, Ariyawansa HA et al (2015) Fungal diversity notes 1-110: taxonomic and phylogenetic contributions to fungal species. Fungal Divers 72:1-197

Liu J, Zhang D, Zhang M, Liu X et al (2016) Periconiasins I and J, two new cytochalasans from an endophytic fungus Periconia sp. Tetrahedron Lett 57:5794-5797

Liu H, Carvalhais LC, Crawford M, Singh E (2017a) Inner plant values: diversity, colonization and benefits from endophytic bacteria. Front Microbiol 8:1-17

Liu J, Zhan D, Zhang M, Chen R et al (2017b) Periconones B-E, new meroterpenoids from endophytic fungus Periconia sp. Chin Chem Lett 28:248-252

Liu S, Lin N, Chen Y, Liang Z et al (2017c) Biocontrol of sugarcane smut disease by interference of fungal sexual mating and hyphal growth using a bacterial isolate. Front Microbiol 8:1-11

Liu Q, Ma H, Zhang Y, Dong C (2018a) Artificial cultivation of true morels: current state, issues and perspectives. Crit Rev Biotechnol 38:259-271

Liu Z, Wang Z, Lv X, Zhu X et al (2018b) Comparison study of the volatile profiles and microbial commnities of Wuyi $\mathrm{Qu}$ and Gutian Qu, two major types of traditional fermentation starters of Hong Qu glutinous rice wine. Food Microbiol 69:105-115 
Liuzzi VC, Mirabelli V, Cimmarusti MT, Haidukowski M (2017) Enniatin and beauvericin biosynthesis in Fusarium species: production profiles and structural determinant prediction. Toxins (Basel) 9:1-17

Livne-Luzon S, Ovadia O, Weber G, Avidan Y et al (2017) Smallscale spatial variability in the distribution of ectomycorrhizal fungi affects plant performance and fungal diversity. Ecol Lett 20:1192-1202

Llarena-Hernández RC, Largeteau M, Farnet AM, Minvielle N et al (2011) Phenotypic variability in cultivars and wild strains of Agaricus brasiliensis and Agaricus subrufescens. In: Proceedings from 7th international conference on mushroom biology and mushroom products, Bordeaux, France, pp 38-49

Llarena-Hernández RC, Largeteau ML, Farnet AM, Foulongne-Oriol M (2013) Potential of European wild strains of Agaricus subrufescens for productivity and quality on wheat straw based compost. World J Microbiol Biotechnol 29:1243-1253

Llarena-Hernández CR, Largeteau ML, Ferrer N, Regnault-Roger C, Savoie JM (2014) Optimization of the cultivation conditions for mushroom production with European wild strains of Agaricus subrufescens and Brazilian cultivars. J Sci Food Agric 94:77-84

Lo HC, Tsai FA, Wasser SP, Yang JG, Huang BM (2006) Effects of ingested fruiting bodies, submerged culture biomass, and acidic polysaccharide glucuronoxylomannan of Tremella mesenterica Retz.: Fr. on glycemic responses in normal and diabetic rats. Life Sci 78:1957-1966

Loredo-Treviño A, García G, Velasco-Téllez A, Rodríguez-Herrera R, Aguilar CN (2011) Polyurethane foam as substrate for fungal strains. Adv Biochem Biotechnol 2:52-58

Lorenz K, Lai R (2014) Biochar application to soil for climate change mitigation by soil organic carbon sequestration. J Plant Nutr Soil Sci 177:651-670

Lu Z, Kou W, Du B, Wu Y et al (2008) Effect of Xuezhikang, an extract from red yeast Chinese rice, on coronary events in a Chinese population with previous myocardial infarction. Am J Cardiol 101:1689-1693

Lu X, Chen H, Dong P, Fu L, Zhang X (2010) Phytochemical characteristics and hypoglycaemic activity of fraction from mushroom Inonotus obliquus. J Sci Food Agric 90:276-280

Luangharn T, Karunarathna SC, Khan S, Xu JC et al (2017) Antibacterial activity, optimal culture conditions and cultivation of the medicinal Ganoderma australe, new to Thailand. Mycosphere 8:1108-1123

Lucas N, Bienaime C, Belloy C, Queneudec M et al (2008) Polymer biodegradation: mechanisms and estimation techniques: a review. Chemosphere 73:429-442

Lucas JA, Hawkins NJ, Fraaje BA (2015) The evolution of fungicide resistance. Adv Appl Microbiol 90:29-92

Luh BS (1995) Industrial production of soy sauce. J Ind Microbiol 14:467-471

Luna JM, Rufino RD, Sarubbo LA (2016) Biosurfactant from Candida sphaerica UCP0995 exhibiting heavy metal remediation properties. Process Saf Environ 102:558-566

Lwin NW, Yu KH, Thein MM, Lwin T (2012) Screening of sugarcane families for resistance to sugarcane smut. Int Sugar J 114:512-516

López MJ, Nichols NN, Dien BS, Moreno J, Bothast RJ (2004) Isolation of microorganisms for biological detoxification of lignocellulosic hydrolysates. Appl Microbiol Biotechnol 64:125131

López SC, Peng M, Issak TY, Daly P et al (2018) Induction of plant cell wall degrading CAZyme encoding genes by lignocellulosederived monosaccharides and cellobiose in the white-rot fungus Dichomitus squalens. Appl Environ Microbiol 84:e00403e00418
López-Abelairas M, Pallín MÁ, Salvachúa D, Lú-Chau T et al (2013) Optimisation of the biological pretreatment of wheat straw with white-rot fungi for ethanol production. Bioprocess Biosyst Eng 36:1251-1260

López-Sampson A, Page T (2018) History of use and trade of agarwood. Econ Bot 72:107-129

Ma H, Liang K, Zhou Z, Huang G, Lin M (2012) Method for rapid formation of agilawood of agallochum through induction by using fungus fermentation liquid. Chinese patent 102550311A, 11 July 2012

Ma X, Li L, Zhu T, Ba M et al (2013) Phenylspirodrimanes with antiHIV activity from the sponge-derived fungus Stachybotrys chartarum MXHX73. J Nat Prod 76(12):2298-2306

Ma X, Kang J, Nontachaiyapoom S, WenT HK (2015a) Nonmycorrhizal endophytic fungi from orchids. Curr Sci 109:36-51

Ma XL, Meng M, Han LR, Li Z et al (2015b) Immunomodulatory activity of macromolecular polysaccharide isolated from Grifola frondosa. Chin J Nat Med 13:906-914

Maas RH, Bakker RR, Eggink G, Weusthuis RA (2006) Lactic acid production from xylose by the fungus Rhizopus oryzae. Appl Microbiol Biotechnol 72:861-868

Magalhães F, Krogerus K, Vidgren V, Sandell M, Gibson B (2017) Improved cider fermentation performance and quality with newly-generated Saccharomyces cerevisiae $\times$ Saccharomyces eubayanus hybrids. J Ind Microbiol Biotechnol 44:1203-1213

Magnuson JK, Lasure LL (2004) Organic Acid Production by Filamentous Fungi. In: Tkacz JS, Lange L (eds) Advances in fungal biotechnology for industry, agriculture, and medicine. Kluwer Academic/Plenum Publishers, New York, pp 307-340

Mahapatra K, Nanda RK, Bag SS, Banerjee R (2005) Purification, characterization and some studies on secondary structure of tannase from Aspergillus awamori Nakazawa. Process Biochem 40:3251-3254

Mahmud H, Hossain I (2016) Effects of plant extracts, BAUbiofungicide and fungicides on quality and health of seed. Bangladesh J Bot 45:677-684

Maia MMD, Heasley A, Camargo de Morais MM, Melo EHM et al (2001) Effect of culture conditions on lipase production by Fusarium solani in batch fermentation. Bioresour Technol 76:23-27

Makita N, Hirano Y, Yamanaka T, Yoshimura K, Kosugi Y (2012) Ectomycorrhizal-fungal colonization induces physiomorphological change in Quercus serrata leaves and roots. J Plant Nutr Soil Sci 175:900-906

Mamo A, Balasubramanian N (2018) Calf rennet production and its performance optimization. J Appl Nat Sci 10:247-252

Mander LN (2003) Twenty years of gibberellin research. Nat Prod Rep 20:49-69

Mankau R (1980) Biocontrol: fungi as nematode control agent. J Nematol 12:244-252

Mansur M, Arias ME, Copa-Patiño JL, Flärdh M, González AE (2017) The white-rot fungus Pleurotus ostreatus secretes laccase isozymes with different substrate specificities. Mycologia 95:1013-1020

Manzur A, Limón-González M, Favela-Torres E (2004) Biodegradation of physio-chemically treated LDPE by a consortium of filamentous fungi. J Appl Polym Sci 92:265-271

Mapari SA, Thrane U, Meyer AS (2010) Fungal polyketide azaphilone pigments as future natural food colorants? Trends Biotechnol 28:300-307

Marlina L, Sukotjo S, Marsudi S (2015) Potential of oil palm Empty Fruit Bunch (EFB) as media for oyster mushroom, Pleurotus ostreatus cultivation. Procedia Chem 16:427-431

Marques TA, Baldo C, Borsato D, Buzato JB, Celligo MAPC (2014) Production and partial characterization of a thermostable alkaline 
and organic solvent tolerant lipase from Trichoderma atroviride 676. Int J Sci Technol Res 3:77-83

Marrone PG (2014) The market and potential for biopesticides. In: Gross AD, Coats JR, Seiber JN, Duke SO (eds) Biopesticides: state of the art and future opportunities. American Chemical Society, Washington, DC, pp 245-258

Martinez FAC, Balciunas EM, Salgado JM, González JMD et al (2013) Lactic acid properties, applications and production: a review. Trends Food Sci Technol 30:70-83

Martins ES, Silva D, Da Silva R, Gomes E (2002) Solid state production of thermostable pectinases from thermophilic Thermoascus aurantiacus. Process Biochem 37:949-954

Martín JF, Coton M (2016) Blue cheese: microbiota and fungal metabolites. In: Frias J, Martinez-Villaluenga C, Peñas E (eds) Fermented foods in health and disease prevention. Academic Press, Boston, pp 275-303

Martín-Sampedro R, Fillat Ú, Ibarra D, Eugenio ME (2015) Use of new endophytic fungi as pretreatment to enhance enzymatic saccharification of Eucalyptus globulus. Bioresour Technol 196:383-390

Mascarin MG, Lopes R, Delalibera I, Fernandes E et al (2018) Current status and perspectives of fungal entomopathogens used for microbial control of arthropod pests in Brazil. J Invert Pathol. https://doi.org/10.1016/j.jip.2018.01.001

Mathur G, Prasad R (2012) Degradation of polyurethane by Aspergillus flavus (ITCC6051) isolated from soil. Appl Biochem Biotechnol 167:1595-1602

Matsubara M, Lynch JM, De Leij FAAM (2006) A simple screening procedure for selecting fungi with potential for use in the bioremediation of contaminated land. Enzyme Microb Technol 39:1365-1372

Matsuda D, Namatame J, Tomoda H, Kobayashi S et al (2004) New beauveriolides produced by amini acid-supplemented fermentation of Beauveria sp. F0-6979. J Antibiot 57:1-9

Mayzumi F, Okamoto H, Mizuno T (1997) Cultivation of reishi (Ganoderma lucidum). Food Rev Int 13:365-370

McCartney L, Lefsrud M (2018) Protected agriculture in axtreme environments: a review of controlled environment agriculture in tropical, arid, polar, and urban locations. Appl Eng Agric 34:455-473

McFarland LV, Bernasconi P (1993) Saccharomyces boulardii. A review of an innovative biotherapeutic agent. Microb Ecol Health Dis 6:157-171

mCore Panels and Blocks (2018). Ecovative Design, LLC. https:// ecovativedesign.com/pilot-plant. Accessed 28 June 2018

Meier JJ, Bhushan A, Butler AE, Rizza RA, Butler PC (2005) Sustained beta cell apoptosis in patients with long-standing type 1 diabetes: indirect evidence for islet regeneration? Diabetologia 48:2221-2228

Mejía LC, Rojas EI, Maynard Z, Van Bael S et al (2008) Endophytic fungi as biocontrol agents of Theobroma cacao pathogens. Biol Control 46:4-14

Mendez-Espinoza C, Garcia-Nieto E, Esquivel AM, Gonzalez MM et al (2013) Antigenotoxic potential of aqueous extracts from the chanterelle mushroom, Cantharellus cibarius (higher Basidiomycetes) on human mononuclear cell cultures. Int J Med Mushrooms 15:325-332

Mendis S, Puska P, Norrving B (2011) Global atlas on cardiovascular disease prevention and control. In: World Health Organization in collaboration with the World Heart Federation and the World Stroke Organization. World Health Organization, pp 3-18

Mendonça MD, Kasuya MC, Cadorin A, Vieira AJ (2005) Agaricus blazei cultivation for a living in Brazil. In: MushWorld (ed) Mushroom growers' handbook 2: shiitake cultivation. MushWorld-Heineart Inc, Seoul
Mercier J, Jiménez JI (2004) Control of fungal decay of apples and peaches by the biofumigant fungus Muscodor albus. Postharvest Biol Technol 31:1-8

Mercier J, Smilanick JL (2005) Control of green mold and sour rot of stored lemon by biofumigation with Muscodor albus. Biocontrol 32:401-407

Mercier J, Jiménez JI (2009) Demonstration of the biofumigation activity of Muscodor albus againt Rhizoctonia solani in soil and potting mix. Biocontrol 54:797-805

Miller SJ (2001) Emerging mechanisms for secondary cardioprotective effects of statins. Cardiovasc Res 52:5-7

Minagawa K, Kouzuki S, Yoshimoto J, Kawamura Y et al (2002) Stachyflin and acetylstachyflin, novel anti-influenza a virus substances, produced by Stachybotrys sp. RF-7260. I. Isolation, structure elucidation and biological activities. J Antibiot 55:155164

Mitchell AM, Strobel GA, Hess WM, Vargas PN, Ezra D (2008) Muscodor crispans, a novel endophyte from Ananas ananassoides in the Bolivian Amazon. Fungal Divers 31:37-43

Mitchell DA, Krieger N, Berovic M (2010) Solid-state fermentation bioreactors. Springer, Berlin

Mitschke N (2017) Isolation and characterization of secondary metabolites from the basidiomycete Dentipellis fragilis. Master thesis, University of Bremen

Miyake T, Uchitomi K, Zhang MY et al (2006) Effects of the principal nutrients on lovastatin production by Monascus pilosus. Biosci Biotechnol Biochem 70:1154-1159

Miyamoto M, Takano M, Kuwahara M, Soyama H et al (2018) Efficacy of combination chemotherapy using irinotecan and nedaplatin for patients with recurrent and refractory endometrial carcinomas: preliminary analysis and literature review. Cancer Chemother Pharmacol 81:111-117

Miyauchi S, Rancon A, Drula E, Hage H et al (2018) Integrative visual omics of the white-rot fungus Polyporus brumalis exposes the biotechnological potential of its oxidative enzymes for delignifying raw plant biomass. Biotechnol Biofuels 11:201214

Mohamed R, Jong PL, Irdayu IN (2014) Succession patterns of fungi associated to wound-induced agarwood in wild Aquilaria malaccensis revealed from quantitative pcr assay. World $\mathrm{J}$ Microbiol Biotechnol 30:2427-2436

Mohamed R, Rasool S, Mohamed R (eds) (2016) Understanding agarwood formation and its challenges. In: Mohamed R (ed) Agarwood. Springer, Singapore, pp 39-56

Mohd-Nasir H, Mohd-Setapar SH (2018) Natural ingredients in cosmetics from Malaysian plants: a review. Sains Malays 47:951-959

Mohorčič M, Friedrich J, Renimel I, André P et al (2007) Production of melanin bleaching enzyme of fungal origin and its application in cosmetics. Biotechnol Bioprocess Eng 12:200-206

Mohr FB, Lermen C, Gazim ZC, Goncalves JE, Alverton O (2017) Antifungal activity, yield, and composition of Ocimum gratissimum essential oil. Genet Mol Res 16:gmr16019542

Molina FI, Shen P, Jong SC, Orikono K (1992) Molecular evidence supports the separation of Lentinula edodes from Lentinus and related genera. Can J Bot 70:2446-2452

Moncalro AV, Filho EXF (2017) Fungal lytic polysaccharide monooxygenases from family AA9: recent developments and application in lignocelullose breakdown. Int $\mathbf{J}$ Biol Macromol 102:771-778

Monggoot S, Popluechai S, Gentekaki E, Pripdeevech P (2017) Fungal endophytes: an alternative source for production of volatile compounds from agarwood oil of Aquilaria subintegra. Microb Ecol 74:54-61 
Moniruzzaman M (1995) Alcohol fermentation of enzymatic hydrolysate of exploded rice straw by Pichia stipitis. World J Microbiol Biotechnol 11:646-648

Moon B, Lo YM (2014) Conventional and novel applications of edible mushrooms in today's food industry. J Food Process Preserv 38:2146-2153

Moore D, Chiu SW (2001) Fungal products as food. Bio-exploitation of filamentous fungi. Fungal Diversity Press, Hong Kong, pp 223-251

Moore D, Robson GD, Trinci AP (2000) 21st century guidebook to fungi. Cambridge University Press, Cambridge

Moosavi MR (2014) Dynamics of damage to eggplant by Meloidogyne javanica. J Zool 3:43-49

Moosavi MR, Zare R, Zamanizadeh HR, Fatemy S (2011) Pathogenicity of Verticillium epiphytum isolates against Meloidogyne javanica. Int J Pest Manag 57:291-297

Morales-Oyervides L, Oliveira J, Sousa-Gallagher M, Méndez-Zavala A, Montañez JC (2017) Assessment of the dyeing properties of the pigments produced by Talaromyces spp. J Fungi 3:38

Morel S, Arnould S, Vitou M, Boudard F et al (2018) Antiproliferative and antioxidant activities of wild Boletales mushrooms from France. Intern J Med Mushrooms 20:13-29

Morgan-Jones G, McKemy JM (1990) Studies in the genus Cladosporium sensu lato. I. Concerning Cladosporium uredinicola, occurring on telial columns of Cronartium quercuum and other rusts. Mycotaxon 39:185-200

Moricca S, Ragazzi A, Mitchelson KR (1999) Molecular and conventional detection and identification of Cladosporium tenuissimum on two-needle pine rust aeciospores. Can J Bot 77:339-347

Morris MJ (1991) The use of plant pathogens for biological weed control in South Africa. Agric Ecosyst Environ 37:239-255

Moschopoulou E (2017) Microbial milk coagulants. In: Ray RC, Rosell CM (eds) Microbial enzyme technology in food applications. CRC Press, Boca Raton, pp 199-312

Mosedale JR, Puech JL, Feuillat F (1999) The influence on wine flavor of the oak species and natural variation of heartwood components. Am J Enol Viticult 50:503-512

Moslehi-Jenabian S, Lindegaard L, Jespersen L (2010) Beneficial effects of probiotic and food borne yeasts on human health. Nutrients 2:449-473

Movassaghi M, Piizzi G, Siegel DS, Piersanti G (2006) Enantioselective total synthesis of (-)-acylfulvene and (-)-irofulven. Angew Chem Int Ed 45:5859-5863

Mubyana-John T, Wright MG (2011) Fungi and their use in the possible control of nematodes in Botswana soils. Afr J Plant Sci $5: 33-40$

Mudalungu CM, Richter C, Wittstein K, Ali Abdalla M et al (2016) Laxitextines $\mathrm{A}$ and $\mathrm{B}$, cyathane xylosides from the tropical fungus Laxitextum incrustatum. J Nat Prod 79:894-898

Mueller P (2018) US patent application 62:675,922. U.S. Patent and Trademark Office, Washington, DC

Muhammad H, Khan SA, Khan AL, Rehman G et al (2009) Phoma herbarum as a new gibberellin-producing and plant growthpromoting fungus. J Microbiol Biotechnol 19:1244-1249

Munusamy U, Sabaratnam V, Muniandy S, Abdullah N et al (2008) Biodegradation of polycyclic aromatic hydrocarbons by laccase of Pycnoporus sanguineus and toxicity evaluation of treated PAH. Biotechnology 7:669-677

Murali M, Amruthesh KN (2015) Plant growth-promoting fungus Penicillium oxalicum enhances plant growth and induces resistance in pearl millet against downy mildew disease. J Phytol 163:743-754

Murali M, Amruthesh KN, Sudisha J, Niranjana SR, Shetty HS (2012) Screening for plant growth promoting fungi and their ability for growth promotion and induction of resistance in pearl millet against downy mildew disease. J Phytol 4:30-36

Myronycheva O, Bandura I, Bisko N, Gryganskyi AP, Karlsson O (2017) Assessment of the growth and fruiting of 19 oyster mushroom strains for indoor cultivation on lignocellulosic wastes. BioResources 12:4606-4626

Naef R (2011) The volatile and semi-volatile constituents of agarwood, the infected heartwood of Aquilaria species. Flavour Fragr J 26:73-87

Nagia FA, El-Mohamedy RSR (2007) Dyeing of wool with natural anthraquinone dyes from Fusarium oxysporum. Dyes Pigments 75:550-555

Nagy B, Măicăneanu A, Indolean C, Mânzatu C, Silaghi-Dumitrescu MC (2013) Comparative study of Cd(II) biosorption on cultivated Agaricus bisporus and wild Lactarius piperatus based biocomposites. Linear and nonlinear equilibrium modelling and kinetics. J Taiwan Inst Chem Eng. https://doi.org/10.1016/j.jtice. 2013.08.013

Namatame I, Tomoda H, Tabata N, Si SY, Omura S (1999) Structure elucidation of fungal Beauveriolide-III, a novel inhibitor of lipid droplet formation in mouse macrophages. J Antibiot 52:7-12

Naqqash MN, Gökçe A, Bakhsh A, Salim M (2016) Insecticide resistance and its molecular basis in urban insect pests. Parasitol Res 115:1363-1373

Narmani A, Teponno RB, Arzanlou M, Surup F et al (2019) Cytotoxic, antimicrobial and antiviral secondary metabolites produced by the plant pathogenic fungus Cytospora sp. CCTU A309. Fitoterapia 134:314-322

Narusaka Y, Nakashima K, Shinwari ZK, Sakuma Y et al (2003) Interaction between two cis-acting elements, ABRE and DRE, in ABA-dependent expression of Arabidopsis rd29A gene in response to dehydration and high-salinity stresses. Plant J 34:137-148

Nash AK, Auchtung TA, Wong MC, Smith DP et al (2017) The gut mycobiome of the human microbiome project healthy cohort. Microbiome 5:153

Newton GGF, Abraham EP (1955) Cephalosporin C, a new antibiotic containing sulphur and D- $\alpha$-aminoadipic Acid. Nature 175:548

Nguyen TB (2004) Mushroom growers' handbook1, Oyster mushroom cultivation Part II. Oyster mushrooms, chapter 5 substrate Rubber tree sawdust. Biology Institute, Dalat, pp 176-179

Nguyen VD, Kosuge H, Auresenia J, Tan R, Brondial Y (2009) Effect of vacuum pressure on ethanol fermentation. J Appl Sci 17:3020-3026

Nieto-Domínguez M, de Eugenio LI, York-Durán MJ, RodríguezColinas B et al (2017) Prebiotic effect of xylooligosaccharides produced from birchwood xylan by a novel fungal GH11 xylanase. Food Chem 232:105-113

Nijssen B (1991) Off-Flavors. In: Maarse H (ed) Volatile compounds in foods and beverages, 1st edn. Marcel Dekker, Inc., New York, pp 689-735

Nitao JK, Meyer SLF, Oliver JE, Schmidt W, Chitwood D (2002) Isolation of flavipin, a fungus compound antagonistic to plantparasitic nematodes. Nematology 4:55-63

Nofiani R, de Mattos-Shipley K, Lebe KE et al (2018) Strobilurin biosynthesis in basidiomycete fungi. Nat Commun 9:3940

Nontachaiyapoom S, Sasirat S, Manoch L (2010) Isolation and identification of Rhizoctonia-like fungi from roots of three orchid genera, Paphiopedilum, Dendrobium, and Cymbidium, collected in Chiang Rai and Chiang Mai provinces of Thailand. Mycorrhiza 20:459-471

Nordbring-Hertz B, Jansson HB, Tunlid A (2011) Nematophagous fungi. In: eLS. Wiley, Chichester

Norris RF (1992) Have ecological and biological studies improved weed control strategies? In: Proceedings of the 7th Australian weeds conference, vol 1, p 129 
Nout MJR, Kiers JL (2005) Tempe fermentation, innovation and functionality: update into the third millenium. J Appl Microbiol 98:789-805

Novak R, Shlaes DM (2010) The pleuromutilin antibiotics: a new class for human use. Curr Opin Investig Drugs 11:182-191

Novotný Č, Svobodová K, Erbanová P, Cajthaml T, Kasinath A, Lang E, Šašek V (2004) Ligninolytic fungi in bioremediation: extracellular enzyme production and degradation rate. Soil Biol Biochem 36:1545-1551

Novriyanti E, Santosa E, Syafii W, Turjaman M, Sitepu IR (2010) Anti fungal activity of wood extract of Aquilaria crassna Pierre ex Lecomte against agarwood-inducing fungi, Fusarium solani. Indones J For Res 7:155-165

Numponsak T, Kumla J, Suwannarach N, Matsui K, Lumyong S (2018) Biosynthetic pathway and optimal conditions for the production of indole-3-acetic acid by an endophytic fungus, Colletotrichum fructicola CMU-A109. PLoS ONE 13(10): e0205070

Nwuche CO, Ogbonna JC (2011) Isolation of lipase producing fungi from palm oil mill effluent (POME) dump sites at Nsukka. Braz Arch Biol Technol 54:113-116

Nyakundi WO, Magoma G, Ochora J, Nyende AB (2011) Biodegradation of diazinon and methomyl pesticides by white rot fungi from selected horticultural farms in rift valley and Central Kenya. J Appl Technol Environ Sanit 1:107-1249

Obi FO, Ugwuishiwu BO, Nwakaire JN (2016) Agricultural waste concept, generation, utilization and management. Niger J Technol 35:957-964

Odinot E, Fine F, Sigoillot JC, Navarro D, Laguna O, Bisotto A, Peyronnet C, Ginies C, Lecomte J, Faulds CB, Lomascolo A (2017) A two-step bioconversion process for canolol production from rapeseed meal combining an Aspergillus niger feruloyl esterase and the fungus Neolentinus lepideus. Microorganisms 5:67

Oei P (2005) Small-scale mushroom cultivation of oyster. Shiitake and wood ear mushrooms. Digigrafi, Wageningen

Ogel ZB (2018) Microbial production of recombinant rennet: recent developments. Microbial cultures and enzymes in dairy technology. IGI Global, Hershey, pp 222-233

Ohshiro T, Namatame I, Nagai K, Sekiguchi T et al (2006) Absolute stereochemistry of fungal beauveriolide III and ACAT inhibitory activity of four stereoisomers. J Org Chem 71:7643-7649

Okamura T, Ogata T, Minamimoto N, Takeno T et al (2001) Characteristics of beer-like drink produced by mushroom fermentation. J Jpn Soc Food Sci 7:88-90

Okamura-Matsui T, Takemura K, Sera M, Takeno T et al (2001) Characteristics of a cheese-like food produced by fermentation of the mushroom Schizophyllum commune. J Biosci Bioeng 92:30-32

Okamura-Matsui T, Tomada T, Fukuda S, Ohsugi M (2003) Discovery of alcohol dehydrogenase from mushrooms and application to alcoholic beverages. J Mol Catal B 23:133-144

Olivain C, Alabouvette C, Steinberg C (2004) Production of a mixed inoculum of Fusarium oxysporum Fo47 and Pseudomonas fluorescens $\mathrm{C} 7$ to control Fusarium diseases. Biocontrol Sci Technol 14:227-238

Olufolaji DB (1993) Evaluation of some relatively new fungicides for smut control in sugar-cane. Crop Prot 12:293-295

Olusola SA, Anslem EE (2010) Bioremediation of a crude oil polluted soil with Pleurotus pulmonarius and Glomus mosseae using Amaranthus hybridus as a test plant. J Bioremed Biodegrad $1: 111$

Oono R, Lefèvre E, Simha A, Lutzoni F (2015) A comparison of the community diversity of foliar fungal endophytes between seedling and adult loblolly pines (Pinus taeda). Fungal Biol 119:917-928
Ouwehand AC, Salminen S, Isolauri E (2002) Probiotics: an overview of beneficial effects. In: Lactic acid bacteria: genetics, metabolism and applications, pp. 279-289

Oyserman BO, Medema MH, Raaijmakers JM (2018) Road MAPs to engineer host microbiomes. Curr Opin Microbiol 43:46-54

O'Connell PJ, Zoschke A (1996) Limitations to the development and commercialisation of mycoherbicides by industry. In: Proceedings of the 2nd international weed control congress, 27-28 June 1996, Copenhagen, Denmark, pp 1189-1195

O'Hanlon KA, Knorr K, Jorgensen LN, Nicolaisen M, Boelt B (2012) Exploring the potential of symbiotic fungal endophytes in cereal disease suppression. Biol Control 63:69-78

Pachauri P, Aranganathan V, More S, Sullia SB, Deshmukh S (2017) Purification and characterization of cellulase from a novel isolate of Trichoderma longibrachiatum. Biofuels 1:7. https://doi.org/ 10.1080/17597269.2017.1345357

Padma VV (2015) An overview of targeted cancer therapy. BioMedicine 5:19

Pal S, Singh HB, Farooqui A, Rakshit A (2015) Fungal biofertilizers in Indian agriculture: perception, demand and promotion. J Ecofriendly Agric 10:101-113

Palazzolo E, Saiano F, Laudicina VA, Gargano ML, Venturella G (2017) Volatile organic compounds in wild fungi from Mediterranean forest ecosystems. J Essent Oil Res 29:385-389

Palmqvist E, Hahn-Hägerdal B (2000) Fermentation of lignocellulosic hydrolysates. II: inhibitors and mechanisms of inhibition. Bioresour Technol 74:25-33

Panagiotidou E, Konidaris C, Baklavaridis A, Zuburtikudis I et al (2014) A simple route for purifying extracellular poly(3hydroxybutyrate)-depolymerase from Penicillium pinophilum. Enzyme Res 2014:159809

Pandey P, Pathak H, Dave S (2016) Microbial ecology of hydrocarbon degradation in the soil: a review. Res J Environ Toxicol 10:1-15

Pandya U, Dhuldhaj U, Sahay NS (2018) Bioactive mushroom polysaccharides as antitumor: an overview. Nat Prod Res. https:// doi.org/10.1080/14786419.2018.1466129

Pang X, Zhao J, Fang X, Zhang T et al (2017) Metabolites from the plant endophytic fungus Aspergillus sp. CPCC 400735 and their anti-HIV activities. J Nat Prod 80:2595-2601

Pansuriya RC, Singhal RS (2010) Response surface methodology for optimization of production of lovastatin by solid state fermentation. Braz J Microbiol 41:164-172

Papagianni M (2007) Advances in citric acid fermentation by Aspergillus niger: biochemical aspects, membrane transport and modeling. Biotechnol Adv 25:244-263

Parafati L, Vitale A, Restuccia C, Cirvilleri G (2015) Biocontrol ability and action mechanism of food-isolated yeast strains against Botrytis cinerea causing post-harvest bunch rot of table grape. Food Microbiol 47:85-92

Park MS, Ahn J, Choi GJ, Choi YH et al (2010) Potential of the volatile-producing fungus Nodulisporium sp. CF016 for the control of postharvest diseases of apple. Plant Pathol J 26:253259

Park JI, Steen EJ, Burd H, Evans SS et al (2012) A thermophilic ionic liquid-tolerant cellulase cocktail for the production of cellulosic biofuels. PLoS ONE 7:e37010

Park KH, Liu Z, Park CS, Ni L (2016) Microbiota associated with the starter cultures and brewing process of traditional Hong $\mathrm{Qu}$ glutinous rice wine. Food Sci Biotechnol 25:649-658

Patel AV, Jakobs-Schonwandt D, Rose T, Vorlop KD (2011) Fermentation and microencapsulation of the nematophagous fungus Hirsutella rhossiliensis in a novel type of hollow beads. Appl Microbiol Biotechnol 89:1751-1760 
Pathmashini L, Arulnandhy V, Wijeratnam WRS (2008) Cultivation of Oyster mushroom (Pleurotus ostreatus) on sawdust. Cey J Sci (Bio Sci) 37:177-182

Patil BD, Jadhav SW, Kakade SS (1989) Studies on cultivation of Pleurotus sajor-caju on different substrates. J Maharashtra Agric Univ 14:156-158

Patkar RN, Naqvi NI (2017) Fungal manipulation of hormoneregulated plant defense. PLoS Pathog 13(6):e1006334

Pavlineri N, Skoulikidis N, Tsihrintzis V (2017) Constructed floating wetlands: a review of research, design, operation and management aspects, and data meta-analysis. Chem Eng J 308:11201132

Peery AF, Crockett SD, Barritt AS, Dellon ES et al (2015) Burden of gastrointestinal, liver and pancreatic diseases in the United States. Gastroenterology 149:1731-1741

Pegklidou K, Mantzouridou F, Tsimidou MZ (2008) Lycopene production using Blakeslea trispora in the presence of 2-methyl imidazole: yield, selectivity, and safety aspects. J Agric Food Chem 56:4482-4490

Pejin BK, Jovanovic K, Mojovic MG, Savic A (2013) New and highly potent antitumor natural products from marine-derived fungi: covering the period from 2003 to 2012. Curr Top Med Chem 13:2745-2766

Pelletier MG, Holta GA, Wanjura JD, Bayer E, McIntyre G (2013) An evaluation study of mycelium based acoustic absorbers grown on agricultural by-product substrates. Ind Crop Prod 51:480-485

Peláez F, Cabello A, Platas G, Díez MT et al (2000) The discovery of enfumafungin, a novel antifungal compound produced by an endophytic Hormonema species biological activity and taxonomy of the producing organisms. Syst Appl Microbiol 23:333343

Peng R, Xiong A, Xue Y, Fu X (2008) Microbial biodegradation of polyaromatic hydrocarbons. FEMS Microbiol Rev 32:927-955

Peng CS, Osman MF, Bahari N, Zakaria R, Rahim KA (2015a) Agarwood inducement technology: a method for producing oil grade agarwood in cultivated Aquilaria malaccensis Lamk. J Agrobiotechnol 6:1-16

Peng WF, Wang X, Hong Z, Zhu GX et al (2015b) The antidepression effect of Xylaria nigripes in patients with epilepsy: a multicenter randomized double-blind study. Seizure 29:26-33

Peng M, Aguilar-Pontes MV, Hainaut M, Henrissat B et al (2018) Comparative analysis of basidiomycete transcriptomes reveals a core set of expressed genes encoding plant biomass degrading enzymes. Fungal Genet Biol 112:40-46

Pengnoi P, Mahawan R, Khanongnuch C, Lumyong S (2017) Antioxidant properties and production of monacolin $\mathrm{K}$, citrinin, and red pigments during solid state fermentation of purple rice (Oryza sativa) varieties by Monascus purpureus. Czech J Food Sci 35:32-39

Peters S, Spiteller P (2007) Sanguinones A and B, blue pyrroloquinoline alkaloids from the fruiting bodies of the mushroom Mycena sanguinolenta. J Nat Prod 70:1274-1277

Petracek PD, Silverman FP, Greene DW (2003) A history of commercial plant growth regulators in apple production. HortScience 38:937-942

Petrelli F, Borgonovo K, Cabiddu M, Lonati V, Barni S (2012) Relationship between skin rash and outcome in non-small-cell lung cancer patients treated with anti-EGFR tyrosine kinase inhibitors: a literature-based meta-analysis of 24 trials. Lung Cancer 78:8-15

Phan CW, Sabaratnam V (2012) Potential uses of spent mushroom substrate and its associated lignocellulosic enzymes. Appl Microbiol Biotechnol 96:863-873

Phan CW, Wong WL, David P, Naidu M, Sabaratnam V (2012) Pleurotus giganteus (Berk.) Karunarathna \& KD Hyde:
Nutritional value and in vitro neurite outgrowth activity in rat pheochromocytoma cells. BMC Complement Altern Med 12:102

Philippe J, Raccah D (2009) Treating type 2 diabetes: how safe are current therapeutic agents? Int J Clin Pract 63:321-332

Philippoussis A, Diamantopoulou P, Israilides C (2007) Productivity of agricultural residues used for the cultivation of the medicinal fungus Lentinula edodes. Int Biodeter Biodegr 59:216-219

Phillips BJ, Strobel GA, Dirkse E, Ezre D, Castillo U (2010) Method of using endophytic fungi to decontaminate and decompose human and animal wastes. US patent 7,858,362B2

Phukhamsakda C, Macabeo APG, Yuyama K, Hyde KD, Stadler M (2018) Biofilm inhibitory abscisic acid derivatives from the plant-associated Dothideomycete fungus, Roussoella sp. Molecules 23:2190

Pineda A, Zheng SJ, van Loon JJA, Pieterse CMJ, Dicke M (2010) Helping plants to deal with insects: the role of beneficial soilborne microbes. Trends Plant Sci 15:507-514

PlasticsEurope (2017) Plastics - the facts 2017. An analysis of European plastics production, demand and waste data for 2017. PlasticsEurope, Brussels. http://www.plasticseurope.org.

Poinar GO Jr, Georgis R (1994) Biological control for plant-parasitic nematodes attacking turf and ornamentals. In: Handbook of integrated pest management for turf and ornamentals, pp 491501

Pointing SB (2001) Feasibility of bioremediation by white-rot fungi. Appl Microbiol Biotechnol 51:20-33

Pointing SB, Hyde KD (2001) Bio-exploitation of filamentous fungi. Fungal Diversity Press, Hong Kong.

Pointing SB, Pelling AL, Smith GJD, Hyde KD, Reddy CA (2001) Screening of basidiomycetes and xylariaceous fungi for lignin peroxidase and laccase gene-specific sequences. Mycol Res 109:115-124

Politowicz J, Lech K, Lipan L, Figiel A, Carbonell-Barrachina ÁA (2018) Volatile composition and sensory profile of shiitake mushrooms as affected by drying method. J Sci Food Agric 98:1511-1521

Poornima K, Preetha R (2017) Biosynthesis of food flavors and fragrances-a review. Asian J Chem 29:2345-2352

Poorniammal R, Parthiban M, Gunasekaran S, Murugesan R, Thilagavathy R (2013) Natural dye production from Thermomyces sp. fungi for textile application. Indian J Fiber Text Res 38:276-279

Poucheret P, Fons F, Rapior S (2006) Biological and pharmacological activity of higher fungi: 20-year retrospective analysis. Cryptogam, Mycol 27:311-333

Powell JM (1971) Fungi and bacteria associated with Cronartium comandrae on lodgepole pine in Alberta. Phytoprotection 52:4551

Powis G, Wipf P, Lynch SM, Birmingham A, Kirkpatrick DL (2006) Molecular pharmacology and antitumor activity of palmarumycin-based inhibitors of thioredoxin reductase. Mol Cancer Ther 5:630-636

Prateep A, Sumkhemthong S, Suksomtip M, Chanvorachote P, Chaotham C (2017) Peptides extracted from edible mushroom: Lentinus squarrosulus induces apoptosis in human lung cancer cells. Pharm Biol 55:17-92

Premalatha K, Kalra A (2013) Molecular phylogenetic identification of endophytic fungi isolated from resinous and healthy wood of Aquilaria malaccensis, a red listed and highly exploited medicinal tree. Fungal Ecol 6:205-211

Prestinaci F, Pezzotti P, Pantosti A (2015) Antimicrobial resistance: a global multifaceted phenomenon. Pathog Glob Health 109:309_ 318

Preuss HG, Echard B, Bagchi D, Perricone NV, Zhuang C (2007) Enhanced insulin-hypoglycemic activity in rats consuming a 
specific glycoprotein extracted from maitake mushroom. Mol Cell Biochem 306:105-113

Punja ZK, Raj SU (2003) Using fungi and yeasts to manage vegetable crop diseases. Trends Biotechnol 21:400-407

Puntambekar US (1995) Cellulase production by the edible mushroom Volvariella diplasia. World J Microbiol Biotechnol 11:695

Purahong W, Hyde KD (2011) Effects of fungal endophytes on grass and non-grass litter decomposition rates. Fungal Divers 47:1-7

Puspoki Z, Storath M, Sage D, Unser M (2016) Transforms and operators for directional bioimage analysis: a survey, Chap. 3. In: De Vos WH, Munck S, Timmermans J-P (eds) Advances in anatomy, embryology and cell biology. Focus on bio-image informatics, vol 219. Springer, Berlin, pp 69-93

Qambrani NA, Rahman MM, Won S, Shim S, Ra C (2017) Biochar properties and eco-friendly applications for climate change mitigation, waste management, and wastewater treatment: a review. Renew Sust Energ Rev 79:255-273

Qian Y, Wang DF, Fan MH, Xu Y et al (2018) Effects of intrinsic metal ions of lentinan with different molecular weights from Lentinus edodes on the antioxidant capacity and activity against proliferation of cancer cells. Int J Biol Macromol 120:73-81

Qiao Y, Yang Y, Dong X, Qiu M (2005) ${ }^{13} \mathrm{C}$ NMR in the application of new Ganoderma triterpenoids. J Spectrosc 22:437-456

Quesada-Moraga E, Santos-Quirós R, Valverde-García P, SantiagoÁlvarez C (2004) Virulence, horizontal transmission and sublethal reproductive effects of Metarhizium anisopliae (Anamorphic fungi) on the German cockroach (Blattodea: Blattellidae). J Invert Pathol 87:51-58

Quilliam RS, Glanville HC, Wade SC, Jones DL (2013) Life in the 'charosphere'-does biochar in agricultural soil provide a significant habitat for microorganisms? Soil Biol Biochem 65:287-293

Rademacher W (2015) Plant growth regulators: backgrounds and uses in plant production. J Plant Growth Regul 34:845-872

Radman R, Saez T, Bucke C, Keshavarz T (2003) Elicitation of plants and microbial cell systems. Biotechnol Appl Biochem 37:91102

Radzi SM, Mustafa WAF, Othman SS, Noor HM (2011) Green synthesis of butyl acetate: a pineapple flavor via lipase catalyzed reaction. WASET 59:677-680

Rahi P, Vyas P, Sharma S, Gulati A, Gulati A (2009) Plant growth promoting potential of the fungus Discosia sp. FIHB 571 from tea rhizosphere tested on chickpea, maize and pea. Indian $\mathbf{J}$ Microbiol 49:128-133

Rai M, Varma A (2011) Diversity biotechnology of ectomycorrhizae. Springer, Berlin

Ramachandra RS, Ravishankar GA (2000) Vanilla flavour: production by conventional and biotechnological routes. J Sci Food Agric 80:289-304

Ramachela K, Theron JM (2010) Effect of ectomycorrhizal fungi in the protection of Uapaca kirkiana seedlings against root pathogens in Zimbabwe. South Forests 72:37-45

Ramin AA, Braun PG, Prange RK, DeLong JM (2005) In vitro effects of Muscodor albus and three volatile components on growth of selected postharvest microorganisms. HortScience 40:21092114

Rapior S, Cavalié S, Croze P, Andary C et al (1996) Volatile components of ten frozen mushrooms (Basidiomycetes). J Essent Oil Res 8:63-66

Rapior S, Breheret S, Talou T, Bessière JM (1997a) Volatile flavour constituents of fresh Marasmius alliaceus (garlic Marasmius). J Agric Food Chem 45:820-825

Rapior S, Fruchier A, Bessière JM (1997b) Volatile aroma constituents of agarics and boletes. Recent Res Dev Phytochem $1: 567-584$
Rapior S, Marion C, Pélissier Y, Bessière JM (1997c) Volatile composition of fourteen species of fresh wild mushrooms (Boletales). J Essen Oil Res 9:231-234

Rapior S, Breheret S, Talou T, Pélissier Y, Bessiere J-M (2002) The anise-like odor of Clitocybe odora, Lentinellus cochleatus and Agaricus essettei. Mycologia 94:373-376

Ravasio D, Walther A, Trost K, Vrhovsek U, Wendland J (2014) An indirect assay for volatile compound production in yeast strains. Sci Rep 4:3707

Ravindran B, Wong JWC, Selvam A, Sekaran G (2016) Influence of microbial diversity and plant growth hormones in compost and vermicompost from fermented tannery waste. Bioresour Technol 217:200-204

Rayss R (1943) Contribution a l'étude de deuteromycètes de Palestine. Palest J Bot 3:22-51

Reddy C, Mathew Z (2001) Bioremediation potential of white rot fungi. In: Gadd $\mathrm{G}$ (ed) Fungi in bioremediation. Cambridge University Press, Cambridge

Reddy MS, Murali TS, Suryanarayanan TS, Govinda Rajulu MB, Thirunavukkarasu N (2016) Pestalotiopsis species occur as generalist endophytes in trees of Western Ghats forests of southern India. Fungal Ecol 24:70-75

Redman RS, Kim YO, Woodward CJ, Greer C et al (2011) Increased fitness of rice plants to abiotic stress via habitat adapted symbiosis: a strategy for mitigating impacts of climate change. PLoS ONE 6:e14823

Reis FS, Martins A, Vasconcelos MH, Morales P, Ferreira IC (2017) Functional foods based on extracts or compounds derived from mushrooms. Trends Food Sci Technol 66:48-62

Reiter N, Whitfield J, Pollard G, Bedggood W et al (2016) Orchid reintroductions: an evaluation of success and ecological considerations using key comparative studies from Australia. Plant Ecol 217:81-95

Research and Markets (2018) Global mushroom market size, market share, application analysis, regional outlook, growth trends, key players, competitive strategies and forecasts, 2018 to 2026. Report ID: 4620326. www.researchandmarkets.com

Restrep-Liano M, Osorio NW, León JD (2014) Assessment of the effectiveness of ectomycorrhizal inocula to promote growth and root ectomycorrhizal colonization in Pinus patula seedlings using the most probable number technique. Appl Environ Soil Sci. https://doi.org/10.1155/2014/870616

Restrepo-Flórez JM, Bassi A, Thompson MR (2014) Microbial degradation and deterioration of polyethylene-a review. Int Biodeterior Biodegrad 88:83-90

Reverberi M, Fabbri AA, Zjalic S, Ricelli A et al (2005) Antioxidant enzymes stimulation in Aspergillus parasiticus by Lentinula edodes inhibits aflatoxin production. Appl Microbiol Biotechnol 69:207-215

Rezania S, Din MFM, Taib SM, Sohaili J et al (2017) Review on fermentative biohydrogen production from water hyacinth, wheat straw and rice straw with focus on recent perspectives. Int J Hydrog Energy 42:20955-20969

Reznik SY (1996) Classical biocontrol of weeds in crop rotation: a story of failure and prospects for success. In: Moran VC and Hoffman JH (eds) Proceedings of the IX international symposium on biological control of weeds (Stellenbosch, South Africa), pp 503-506

Rhind JP (2013) Fragrance and wellbeing: Plant aromatics and their influence on the psyche. Singing Dragon, London, p 452

Rho H, Hsieh M, Kandel SL, Cantillo J et al (2018) Do endophytes promote growth of host plants under stress? A meta-analysis on plant stress mitigation by endophytes. Microb Ecol 75:407-418

Rhodes CJ (2013) Applications of bioremediation and phytoremediation. Sci Prog 96:417-427 
Ribeiro B, Andrade PB, Silva BM, Baptista P et al (2008) Comparative study on free amino acid composition of wild edible mushroom species. J Agric Food Chem 56:10973-10979

Ribeiro GO, Gruninger RJ, Badhan A, McAllister TA (2016) Mining the rumen for fibrolytic feed enzymes. Anim Front 6:20-26

Ribes S, Fuentes A, Talens P, Barat JM (2017) Prevention of fungal spoilage in food products using natural compounds: a review. Crit Rev Food Sci Nutr 15:1-5

Richardson KC, Jarett L, Finke EH (1960) Embedding in epoxy resins for ultrathin sectioning in electron microscopy. Stain Technol 35:313-325

Richter C, Wittstein K, Kirk PM, Stadler M (2015) An assessment of the taxonomy and chemotaxonomy of Ganoderma. Fungal Divers 71:1-15

Riga E, Lacey L, Guerra N (2008) Muscodor albus, a potential biocontrol agent against plant-parasitic nematodes of economically important vegetable crops in Washington State, USA. Biocontrol 45:380-385

Rillig MC, Mummey DL (2006) Mycorrhizas and soil structure. New Phytol 171:41-53

Rinaldi AC, Comandini O, Kuyper TW (2008) Ectomycorrhizal fungal diversity: separating the wheat from the chaff. Fungal Divers 33:1-45

Rincão VP, Yamamoto KA, Ricardo NMPS, Soares SA, Meirelles LDP, Nozawa C, Linhares REC (2012) Polysaccharide and extracts from Lentinula edodes: structural features and antiviral activity. Virol J 9:37

Rizal LM, Hyde KD, Chukeatirote E, Karunarathna SC, Kakumyan P, Chamyuang S (2016) First successful cultivation of the edible mushroom Macrolepiota dolichaula in Thailand. Chiang Mai J Sci 43:959-971

Roa Engel CA, Straathof AJJ, Zijlmans TW, van Gulik WM, van der Wielen LAM (2008) Fumaric acid production by fermentation. Appl Microbiol Biotechnol 78:379-389

Robert EH (2010) System for non-deleterious accelerated aging of wine or spirits, p 9

Rodriguez A, Sanders IR (2015) The role of community and population ecology in applying mycorrhizal fungi for improved food security. ISME J 9:1053-1061

Rodriguez RJ, Henson J, van Volkenburgh E, Hoy M et al (2008) Stress tolerance in plants via habitat-adapted symbiosis. ISME J 2:404-416

Rodriguez RJ, White JF Jr, Arnold AE, Redman RS (2009) Fungal endophytes: diversity and functional roles. New Phytol 182:314330

Rodriguez MM, Perez D, Chaves FJ, Esteve E et al (2015) Obesity changes the human gut mycobiome. Sci Rep 5:14600

Rogers JD, Ju YM, Lehmann J (2005) Some Xylaria species on termite nests. Mycologia 97:914-923

Rohlfs M, Churchill AC (2011) Fungal secondary metabolites as modulators of interactions with insects and other arthropods. Fungal Genet Biol 48:23-34

Ronkvist A, Wenchun X, Wenhua L, Richard G (2009) Cutinasecatalyzed hydrolysis of poly(ethylene terephthalate). Macromolecules 42:5128-5138

Ropars J, López-Villavicencio M, Snirc A, Lacoste S, Giraud T (2017) Blue cheese-making has shaped the population genetic structure of the mould Penicillium roqueforti. PLoS ONE 12: $\mathrm{e} 0171387$

Rosmiza MZ, Davies WP, Rosniza Aznie CR, Jabil MJ, Mazdi M (2016) Prospects for increasing commercial mushroom production in Malaysia: challenges and opportunities. Mediterr J Soc Sci 7:406

Rossi MJ, Agenor FJ, de Oliveira VL (2007) Inoculant production of ectomycorrhiza fungi by soild and submerged fermentations. Food Technol Biotechnol 45:277-286
Rosso MD, Cancian D, Panighel A, Vedova AD, Flamini R (2009) Chemical compounds released from five different woods used to make barrels for aging wines and spirits: volatile compounds and polyphenols. Wood Sci Technol 43:375-385

Rothermich MM, Hayes LA, Lovley DR (2002) Anaerobic, sulfatedependent degradation of polycyclic aromatic hydrocarbons in petroleum-contaminated harbor sediment. Environ Sci Technol 36:4811-4817

Rouches E, Zhou S, Sergent M, Raouche S, Carrere H (2018) Influence of white-rot fungus Polyporus brumalis BRFM 985 culture conditions on the pretreatment efficiency for anaerobic digestion of wheat straw. Biomass Bioenerg 110:75-79

Rowe JW, Fulmer T, Fried L (2016) Preparing for better health and health care for an aging population. JAMA 316:1643-1644

Rowley DC, Kelly S, Kauffman CA, Jensen PR, Fenical W (2003) Halovirs A-E, new antiviral agents from a marine-derived fungus of the genus Scytalidium. Bioorg Med Chem 11:4263-4274

Roy HE, Steinkraus DC, Eilenberg J, Hajek AE, Pell JK (2006) Bizarre interactions and endgames: entomopathogenic fungi and their arthropod hosts. Ann Rev Entomol 51:331-357

Royse DJ, Chalupa W (2009) Effects of spawn, supplement and phase II compost additions and time of re-casing second break compost on mushroom (Agaricus bisporus) yield and biological efficiency. Biores Technol 100:5277-5282

Rubner A (1996) Revision of predacious hyphomycetes in the Dactylella-Monacrosporium complex. Stud Mycol 39:1-129

Ruddiman WF, Thomson JS (2001) The case for human causes of increased atmospheric $\mathrm{CH}_{4}$ over the last 5000 years. Quat Sci Rev 20:1769-1777

Rumbold K, van Buijsen HJ, Overkamp KM, van Groenestijn JW et al (2009) Microbial production host selection for converting second-generation feedstocks into bioproducts. Microb Cell Fact 8:64

Rupcic Z, Chepkirui C, Restrepo MH, Crous PW et al (2018) New nematicidal and antimicrobial secondary metabolites from a new species in the new genus, Pseudobambusicola thailandica. MycoKeys 33:1-23

Russell JR, Huang J, Anand P, Kucera K et al (2011) Biodegradation of polyester polyurethane by endophytic fungi. Appl Environ Microbiol 77:6076-6084

Ruxton CH, McMillan B (2010) The impact of mycoprotein on blood cholesterol levels: a pilot study. Brit Food 112:1092-1101

Räisänen R, Björk H, Hynninen PH (2000) Two-dimensional TLC separation and mass spectrometric identification of anthraquinones isolated from the fungus Dermocybe sanguinea. Z Naturforsch 55C:195-202

Sabaratnam V, Phan C-W (2017) Neuroactive components of culinary and medicinal mushrooms with potential to mitigate age-related neurodegenerative diseases. In: Brahmachari G (ed) Discovery and development of neuroprotective agents from natural products. Elsevier, Amsterdam, pp 401-413

Sabarwal A, Kumar K, Singh P (2018) Hazardous effects of chemical pesticides on human health-cancer and other associated disorders. Environ Toxicol Pharmacol 63:103-114

Sacchi KL, Bisson LF, Adams DO (2005) A review of the effect of winemaking techniques on phenolic extraction in red wines. Am J Enol Viticult 56:197-206

Sacramento CQ, Marttorelli A, Fintelman-Rodrigues N, de Freitas CS et al (2015) Aureonitol, a fungi-derived tetrahydrofuran, inhibits influenza replication by targeting its surface glycoprotein hemagglutinin. PLoS ONE 10:e0139236

Saeidnia S, Abdollahi M (2014) Antioxidants: friends or foe in prevention or treatment of cancer: the debate of the century. Toxicol Appl Pharmacol 271:49-63

Sahoo K, Dhal NK, Das R (2014) Production of amylase enzyme from mangrove fungal isolates. Afr J Biotechnol 13:4338-4346 
Saikawa Y, Watanabe T, Hashimoto K, Nakata M (2000) Absolute configuration and tautomeric structure of xylindein, a blue-green pigment of Chlorociboria species. Phytochemistry 55:237-240

Salas-Marina MA, Silva-Flores MA, Cervantes-Badillo MG, RosalesSaavedra MT et al (2011) The plant growth-promoting fungus Aspergillus ustus promotes growth and induces resistance against different lifestyle pathogens in Arabidopsis thaliana. J Microbiol Biotechnol 21:686-696

Saleem A, Ebrahim MKH (2014) Production of amylase by fungi isolated from legume seeds collected in Almadinah Almunawwarah Saudi Arabia. J Taibah Univ Sci 8:90-97

Salmon DP (2012) Neuropsychological features of mild cognitive impairment and preclinical Alzheimer's disease. Curr Top Behav Neurosci 10:187-212

Salmones D, Mata G, Ramos LM, Waliszewski KN (1999) Cultivation of shiitake mushroom, Lentinula edodes, in several lignocellulosic materials originating from the subtropics. Agronomie 19:13-19

Sanda NB, Sunusi M (2016) Fundamentals of biological control of pests. Int J Clin Biol Sci 1:1-11

Sandargo B, Chepkirui C, Cheng T, Chaverra-Muñoz L, Thongbai B, Stadler M, Hüttel S (2019a) Biological and chemical diversity go hand in hand: Basidomycota as source of new pharmaceuticals and agrochemicals. Biotechnol Adv. https://doi.org/10.1016/j. biotechadv.2019.01.011

Sandargo B, Michehl M, Praditya D, Steinmann E, Stadler M, Surup F (2019b) Antiviral meroterpenoid rhodatin and sesquiterpenoids rhodocoranes A-E from the wrinkled peach mushroom, Rhodotus palmatus. Org Lett 21:3286-3289

Sandargo B, Thongbai B, Padutya D, Steinmann E et al (2018) Antiviral 4-hydroxypleurogrisein and antimicrobial pleurotin derivatives from cultures of nematophagous basidiomycete Hohenbuehelia grisea. Molecules 23:2697

Sandhu SS, Shukla H, Shukla S (2017) Biosynthesis of silver nanoparticles by endophytic fungi: Its mechanism, characterization techniques and antimicrobial potential. Afr J Biotechnol 16:683-698

Sangamesh MB, Jambagi S, Vasanthakumari MM, Shetty NJ et al (2017) Thermotolerance of fungal endophytes isolated from plants adapted to the Thar Desert, India. Symbiosis 75:135-147

Sanodiya BS, Thakur GS, Baghel RK, Prasad GB, Bisen PS (2009) Ganoderma lucidum: a potent pharmacological macrofungus. Curr Pharm Biotechnol 10:717-742

Santangelo JS, Turley NE, Johnson MT (2015) Fungal endophytes of Festuca rubra increase in frequency following long-term exclusion of rabbits. Botany 93:233-241

Santerre JP, Labow RS, Duguay DG, Erfle D, Adams GA (1994) Biodegradation evaluation of polyether and polyester urethanes with oxidative and hydrolytic enzymes. J Biomed Mater Res 28:1187-1199

Santos RMDB, Firmino AAP, de Sa CM, Felix CR (1996) Keratinolytic activity of Aspergillus fumigatus Fresenius. Curr Microbiol 33:364-370

Santos A, Sanchez A, Marquina D (2004) Yeasts as biological agents to control Botrytis cinerea. Microbiol Res 159:331-338

Santos CA, Ferreira-Filho JA, O'Donovan A, Gupta VK et al (2017) Production of a recombinant swollenin from Trichoderma harzianum in Escherichia coli and its potential synergistic role in biomass degradation. Microb Cell Fact 16:83

Sarkar SL, Saha P, Sultana N, Akter S (2017) Exploring textile dye from microorganisms, an eco-friendly alternative. Microbiol Res J Int 18:1-9

Sathiyadash K, Muthukumar T, Uma E, Pandey RR (2012) Mycorrhizal association and morphology in orchids. J Plant Interact 7:238-247
Sato H, Nishitoba T, Shirasu S, Oda K, Sakamura S (1986) Ganoderiol A and B, new triterpenoids from the fungus Ganoderma lucidum (Reishi). Agric Biol Chem 50:2887-2890

Sauter H, Steglich W, Anke T (1999) Strobilurins: evolution of new class of active substance (review). Angew Chem Int Ed 38:1329-1349

Sauer M, Porro D, Mattanovich D, Branduardi P (2008) Microbial production of organic acids: expanding the markets. Trends Biotechnol 26:100-108

Sauer M, Porro D, Mattanovich D, Branduardi P (2010) 16 years research on lactic acid production with yeast-ready for the market? Biotechnol Genet Eng Rev 27:229-256

Saxena RK, Malhotra B, Batra A (2005) Commercial importance of some fungal enzymes. In: Arora DK (ed) Handbook of fungal biotechnology, 2nd edn. Marcel Dekker, New York, pp 287-298

Sayyad SA, Panda BP, Javed S, Ali M (2007) Optimization of nutrient parameters for lovastatin production by Monascus purpureus MTCC 369 under submerged fermentation using response surface methodology. Appl Microbiol Biotechnol 73:1054-1058

Schena L, Nigro F, Pentimone I, Ligorio A, Ippolito A (2003) Control of postharvest rots of sweet cherries and table grapes with endophytic isolates of Aureobasidium pullulans. Postharvest Biol Technol 30(3):209-220

Schimel JP, Balser TC, Wallenstein M (2007) Microbial stressresponse physiology and its implications for ecosystem function. Ecology 88:1386-1394

Schlegel M, Dubach V, Buol LV, Sieber TN (2016) Effects of endophytic fungi on the ash dieback pathogen. FEMS Microbiol Ecol 92:142-150

Schüßler A, Schwarzott D, Walker C (2001) A new fungal phylum, the Glomeromycota: phylogeny and evolution. Mycol Res 105:1413-1421

Selosse MA, Schneider-Maunoury L, Taschen E, Rousset F, Richard F (2017) Black Truffle, a hermaphrodite with forced unisexual behaviour. Trends Microbiol 25:784-787

Selvan T, Nandini D, Kaushik PK (2014) Agarwood production for intensive income generation. Rev Res J 3:2-9

Semenova MV, Grishutin SG, Gusakov AV, Okunev ON, Sinitsyn AP (2003) Isolation and properties of pectinases from the fungus Aspergillus japonicus. Biochemistry (Moscow) 68:559-569

Sen S, Talukdar NC, Khan M (2015) A simple metabolite profiling approach reveals critical biomolecular linkages in fragrant agarwood oil production from Aquilaria malaccensis-a traditional agro-based industry in North East India. Curr Sci 108:6371

Sengupta A, Zabala A, Tan SY, Broadstock A et al (2017) Characterization of an ionic liquid-tolerant $\beta$-xylosidase from a marine-derived fungal endophyte. Biochem Cell Biol 95:585591

Senthilkumar S (2003) Mycorrhizal fungi of endangered orchid species in Kolli, a part of Eastern Ghats, South India. Lankesteriana 3:15-156

Senthilkumar N (2007) Biological control of insect pests and diseases of forestry importance. Curr Sci 92:167

Seow SL, Naidu M, David P, Wong KH, Sabaratnam V (2013) Potentiation of neuritogenic activity of medicinal mushrooms in rat pheochromocytoma cells. BMC Compl Alt Med 13:157

Sevindik M, Akgul H, Bal C, Selamoglu Z (2018) Phenolic contents, oxidant/antioxidant potential and heavy metal levels in cyclocybe cylindracea. Indian J Pharm Educ 52:437-441

Shaban DAH, Omaima MS (2010) The utilization of agricultural waste as one of the environmental issues in Egypt (a case study). J Appl Sci Res 6:1116-1124

Shackley S, Carter S, Knowles T, Middelink E et al (2012) Sustainable gasification-biochar systems? A case-study of rice- 
husk gasification in Cambodia, Part I: Context, chemical properties, environmental and health and safety issues. Energy Policy 4:49-58

Shah N, Patel A (2017) Fermented foods: an overview. In: Satyanarayana T, Johri BN, Anil P (eds) Microorganisms in sustainable agriculture, food, and the environment. Apple Academic Press, Cambridge, pp 25-88

Shamina A, Shiva KN, Parthasarathy VA (2007) Food colours of plant origin. CAB Rev Perspect Agric Vet Sci Nutr Nat Resour $2: 1-25$

Shao HJ, Jeong JB, Kim KJ, Lee SH (2015) Anti-inflammatory activity of mushroom-derived hispidin through blocking of NF$\kappa B$ activation. J Sci Food Agric 95:2482-2486

Sharma JK, Heather WA (1980) Effect of Cladosporium aecidiicola Thum. on the viability of urediniospores of Melampsora medusa Thum. in storage. Eur J Plant Pathol 10:360-364

Sharma D, Gupta C, Aggarwal S, Nagpal N (2012) Pigment extraction from fungus for textile dyeing. Indian J Fiber Textile Res 37:65-73

Sharma S, Thakur AD, Sharma S, Atanassova M (2018) Effect of different yeast species on the production of pumpkin based wine. J Inst Brewing 124:187-193

Shashitha KN, Shlini P, Kavitha GS (2016) Vegetable waste-a potent substrate for cultivation of $P$. ostreatus. Int Res Stud Biosci 6:5-9

Shearer CA (1995) Fungal competition. Can J Bot 73(Supp11):S1259_ S1264

Shen Q (2001) Molecular phylogenetic analysis of Grifola frondosa (Maitake) and related species and the influence of selected nutrient supplements on mushroom yield. Plant pathology. The Pennsylvania State University, Pennsylvania

Shen WK (2002) Discussion of the value of intergeneric crosses of Saccharum $\times$ Erianthus. Sugarcane 9:1-5

Shen WK, Deng HH, Li QW, Yang ZD, Jiang ZD (2014) Evaluation of $\mathrm{BC} 1$ and $\mathrm{BC} 2$ from the crossing Erianthus arundinaceus with Saccharum for resistance to sugarcane smut caused by Sporisorium scitamineum. Trop Plant Pathol 39:368-373

Shen Q, Geiser DM, Royse DJ (2017) Molecular phylogenetic analysis of Grifola frondosa (maitake) reveals a species partition separating eastern North American and Asian isolates. Mycologia 94(3):472-483

Shi Y, Xin X, Yuan Q (2012) Improved lycopene production by Blakeslea trispora with isopentenyl compounds and metabolic precursors. Biotechnol Lett 34:849-852

Shi J, Gladden JM, Sathitsuksanoh N, Kambam P et al (2013) Onepot ionic liquid pretreatment and saccharification of switchgrass. Green Chem 15:2579-2589

Shi TQ, Peng H, Zeng SY, Ji RY et al (2017) Microbial production of plant hormones: opportunities and challenges. Bioengineered 8:124-128

Shibamoto T, Yasuhara A, Katami T (2007) Dioxin formation from waste incineration. Rev Environ Contam Toxicol 190:1-41

Shimao M (2001) Biodegradation of plastics. Curr Opin Biotechnol $12: 242-247$

Shiomi K, Matsui R, Isozaki M, Chiba $\mathrm{H}$ et al (2005) Fungal phenalenones inhibit HIV-1 integrase. J Antibiot 58:65-68

Show PL, Oladele KO, Siew QY, Zakry FAA, Lan JC, Ling TC (2015) Overview of citric acid production from Aspergillus niger. Front Life Sci 8:271-283

Shrestha B, Zhang W, Zhang Y, Liu X (2010) What is the Chinese caterpillar fungus Ophiocordyceps sinensis (Ophiocordycipitaceae)? Mycology 1:228-236

Shrivastava S, Varma A (2014) From Piriformospora indica to rootonic: a review. African J Microbiol Res 8:2984-2992
Shuhua S, Li Z, Zhenyuan X, Yinghui Z et al (2002) Study of microorganic nematicide to control tobacco root-knot nematodes. Acta Tabacaria Sinica 8:30-33

Shurtleff W, Aoyagi A (1979) The book of tempeh: professional edition. Harper \& Row, New York

Shushni MAM, Singh R, Mentel R, Lindequist U (2011) Balticolid: a new 12-membered macrolide with antiviral activity from an ascomycetous fungus of marine origin. Mar Drugs 9:844-851

Siddiq M, Ravi R, Sami A (2018) Edible mushrooms: production, processing, and quality. In: Handbook of vegetables and vegetable processing, pp 701-725

Silva D, da Silva ME, da Silva R, Gomes E (2002) Pectinase production by Penicillium viridicatum RFC3 by solid state fermentation using agricultural wastes and agro-industrial byproducts. Braz J Microbiol 33:318-324

Silveira GGD, Oliveira GMD, Ribeiro EJ, Monti R, Contiero J (2005) Microbial rennet produced by Mucor miehei in solid-state and submerged fermentation. Braz Arch Biol Technol 48:931-937

Sinden JW, Hauser E (1950) The nature of the composting process and its relation to short composting. Mushroom Sci 2:123-131

Sinden JW, Hauser E (1953) The short method of composting. Mushroom Sci 1:52-59

Singh H (2006) Mycoremediation: fungal bioremediation. Wiley, Hoboken

Singh L, Singh VP (2011) Microbial decolourization of textile dyes by the fungus Trichoderma harzianum. J Pure Appl Microbiol 6:1829-1833

Singh SB, Zink DL, Goetz MA, Dombrowski AW et al (1998) Equisetin and a novel opposite stereochemical homolog phomasetin, two fungal metabolites as inhibitors of HIV-1 integrase. Tetrahedron Lett 39:2243-2246

Singh SB, Zink DL, Bills GF, Pelaez F et al (2002a) Discovery, structure and HIV-1 integrase inhibitory activities of integracins, novel dimeric alkyl aromatics from Cytonaema sp. Tetrahedron Lett 43:1617-1620

Singh SB, Zink DL, Quamina DS, Pelaez F et al (2002b) Integrastatins: structure and HIV-1 integrase inhibitory activities of two novel racemic tetracyclic aromatic heterocycles produced by two fungal species. Tetrahedron Lett 43:2351-2354

Singh SB, Jayasuriya H, Dewey R, Polishook JD et al (2003a) Isolation, structure, and HIV-1 integrase inhibitory activity of structurally diverse fungal metabolites. J Ind Microbiol Biotechnol 30:721-731

Singh SB, Zink DL, Bills GF, Teran A et al (2003b) Four novel bis(naphtho- $\gamma$-pyrones) isolated from Fusarium species as inhibitors of HIV-1 integrase. Bioorg Med Chem Lett 13:713-717

Singh SB, Zink DL, Guan Z, Collado J et al (2003c) Isolation, structure, and HIV-1 integrase inhibitory activity of xanthoviridicatin $\mathrm{E}$ and $\mathrm{F}$, two novel fungal metabolites produced by Penicillium chrysogenum. Helv Chim Acta 86:3380-3385

Singh SB, Ondeyka JG, Tsipouras N, Ruby C et al (2004) Hinnuliquinone, a $\mathrm{C} 2$-symmetric dimeric non-peptide fungal metabolite inhibitor of HIV-1 protease. Biochem Biophys Res Commun 324:108-113

Singh SK, Strobel GA, Knighton B, Geary B et al (2011) An endophytic Phomopsis sp. possessing bioactivity and fuel potential with its volatile organic compounds. Microb Ecol 61:729-739

Siri-udom S, Suwannarach N, Lumyong S (2017) Applications of volatile compounds acquired from Muscodor heveae against white root rot disease in rubber trees (Hevea brasiliensis Mull. Arg.) and relevant allelopathy effects. Fungal Biol 121:573-581

Smith SE, Read DJ (2008) Mycorrhizal symbiosis, 3rd edn. Academic Press, New York 
Smith SE, Facelli E, Pope S, Smith FA (2010) Plant performance in stressful environments: interpreting new and established knowledge of the roles of arbuscular mycorrhizas. Plant Soil 326:3-20

Smith J, Wherley B, Reynolds C et al (2015) Weed control spectrum and turfgrass tolerance to bioherbicide Phoma macrostoma. Int J Pest Manag 61:91-98

Sobngwi E, Ndour-Mbaye M, Boateng KA, Ramaiya KL (2012) Type 2 diabetes control and complications in specialised diabetes care centres of six sub-Saharan African countries: the Diabcare Africa study. Diabetes Res Clin Pract 95:30-36

Soccol CR, Vandenberghe LPS, Rodrigues C, Pandey A (2006) New perspectives for citric acid production and application. Food Technol Biotechnol 44:141-149

Sohi SP, Krull E, Lopez-Capel E, Bol R (2010) A review of biochar and its use and function in soil. Adv Agron 105:47-82

Solomon KV, Haitjema CH, Henske JK, Gilmore SP (2016) Earlybranching gut fungi possess a large, comprehensive array of biomass-degrading enzymes. Science 351:1192-1195

Sommer C (1996) Bodenbearbeitung. In: Keller ER, Hanus H, Heyland K-U (eds) Handbuch des Pflanzenbaues 1. Eugen Ulmer-Verlag, Stuttgart

Songulashvili G, Elisashvili V, Wasser SP, Nevo E, Hadar Y (2007) Basidiomycetes laccase and manganese peroxidase activity in submerged fermentation of food industry wastes. Enzyme Microb Technol 41:57-61

Souza PM, de Assis Bittencourt ML, Caprara CC, de Freitas M et al (2015) A biotechnology perspective of fungal proteases. Braz J Microbiol 46:337-346

Souza Dias E, Abe C, Schwan RF (2004) Truths and myths about the mushroom Agaricus blazei. Sci Agricola 61:545-549

Souza PM, Magalhaes PO (2010) Application of microbial a-amylase in industry-a review. Braz J Microbiol 41:850-861

Sowerby A, Emmett B, Beier C, Tietema A et al (2005) Microbial community changes in heathland soil communities along a geographical gradient: interaction with climate change manipulations. Soil Biol Biochem 37:1805-1813

Soytong K, Kanokmedhakul S, Kukongviriyapa V, Isobe M (2001) Application of chaetomium species (Ketomium ${ }^{\circledR}$ ) as a new broad spectrum biological fungicide for plant disease control: a review article. Fungal Divers 7:1-15

Spegazzini C (1912) Mycetes argentinenses. Ser. IV. Anales del Museo Nacional de Buenos Aires 23:1-146

Spence CA, Lakshmanan V, Donofrio N, Bais HP (2015) Crucial roles of abscisic acid biogenesis in virulence of rice blast fungus Magnaporthe oryzae. Front Plant Sci 6:1-13

Splivallo R, Fischer U, Göbel C, Feussner I, Karlovsky P (2009) Truffles regulate plant root morphogenesis via the production of auxin and ethylene. Plant Physiol 150:2018-2029

Spokas KA (2010) Review of the stability of biochar in soils: predictability of O:C molar ratios. Carbon Manag 1:289-303

Srilakshmi J, Madhavi J, Lavanya S, Ammani K (2015) Commercial potential of fungal protease: past, present and future prospects. Int J Pharm Chem Biol Sci 2:218-234

Srivastava A, Kar R (2009) Characterization and application of tannase produced by Aspergillus niger ITCC 651407 on pomegranate rind. Braz J Microbiol 40:782-789

Srivastava AK, Defago G, Kern H (1985) Hyperparasitism of Puccinia horiana and other microcyclic rusts. J Phytopathol 114:73-78

Stadler M, Hoffmeister D (2015) Fungal natural products-the mushroom perspective. Front Microbiol 6:127

Stadler M, Mayer A, Anke H, Sterner O (1994) Fatty acid and other compounds with nematicidal activity from cultures of basidiomycetes. Planta Med 60:128-132
Stadler M, Bitzer J, Köpcke B, Reinhardt K, Moldenhauer J (2012) Long chain glycolipids useful to avoid perishing of microbial contamination of materials. US patent 14124429

Stadler M, Læssøe T, Fournier J, Decock C, Schmieschek B, Tichy HV, Peršoh D (2014) A polyphasic taxonomy of Daldinia (Xylariaceae). Stud Mycol 77:1-143

Stajich JE, Harris T, Brunk BP, Brestelli J et al (2012) FungiDB: an integrated functional genomics database for fungi. Nucl Acids Res 40:D675-D681

Stamets P (2000) Growing gourmet and medicinal mushrooms, 3rd edn. Ten Speed Press, Emeryville, p 574

Stamets P (2005) Mycelium running: how mushrooms can help save the world. Ten Speed Press, Berkley

Stamets P, Chilton JS (1983) The mushroom cultivator, a practical guide to growing mushrooms at home. Agorikon Press, Washington

Stangarlin JR, Schulz DG, Franzener G, Assi L et al (2010) Induction of phytoalexins in soybean and sorghum by Saccharomyces boulardii. Arq Inst Biol (Sao Paulo) 77:91-98

Starnes RL, Liu CL, Marrone PG (1993) History, use and future of microbial insecticides. Am Entomologist 39:83-91

Starratta N, Loschiavo SR (1974) The production of aphidicolin by Nigrospora sphaerica. Can J Microbiol 20:416-417

Steiner C, Das KC, Garcia M, Foerster B, Zech W (2008) Charcoal and smoke extract stimulate the soil microbial community in a highly weathered xanthic Ferralsol. Pedobiologia 51:359-366

Steinkraus KH (1995) Handbook of indigenous fermented foods. Dekker, New York

Steyaert RL (1930) Cladosporium hemileiae n. spec. Un parasite de l'Hemileia vastatrix Berk. \& Br. Bull Soc R Bot Belge 63:46-48

Stinson AM, Zidak NK, Strobel GA, Jacobsen BJ (2003) Mycofumigation with Muscodor albus and Muscodor roseus for control of seedling diseases of sugar beet and Verticillium wilt of eggplant. Plant Dis 87:1349-1354

Strader CR, Pearce CJ, Oberlies NH (2011) Fingolimod (FTY720): a recently approved multiple sclerosis drug based on a fungal secondary metabolite. J Nat Prod 74:900-907

Strange RN, Scott PR (2005) Plant disease: a threat to global food security. Annu Rev Phytopathol 43:83-116

Subasinghe S, Hettiarachchi D, Rathnamalala E (2012) Agarwoodtype resin from Gyrinops walla Gaertn: a new discovery. J Trop Ecol 2:43-48

Subhani A, EI-Ghamry AM, Huang C, Xu J (2000) Effect of pesticides (herbicides) on soil microbial biomass. Pak J Biol Sci 3:705-709

Suciatmih Y (2002) Effect of coloring $\mathrm{pH}$ and mordant on fungal dyes quality using woolen yarn. In: AIP conference proceedings inventing prosperous future through biological research and tropical biodiversity management, $\mathrm{p} 020057$

Sumathi T, Viswanath B, Sri Lakshmi A, SaiGopal DVR (2016) Production of Laccase by Cochliobolus sp. isolated from plastic dumped soils and their ability to degrade low molecular weight PVC. Biochem Res Int 2016:1-10

Sun X, Guo LD, Hyde KD (2011) Community composition of endophytic fungi in Acer truncatum and their role in decomposition. Fungal Divers 47:85-95

Sun J, Lin X, Zhou X, Wan J et al (2014) Pestalols A-E, new alkenyl phenol and benzaldehyde derivatives from endophytic fungus Pestalotiopsis sp. AcBC2 isolated from the Chinese mangrove plant Aegiceras corniculatum. J Antibiot 67:451-457

Sun X, Shan R, Li X, Pan J, Liu X, Deng R, Song J (2017) Characterization of 60 types of Chinese biomass waste and resultant biochars in terms of their candidacy for soil application. Gcb Bioenergy 9:1423-1435 
Sung GH, Hywel-Jones NL, Sung JM, Luangsa-Ard JJ, Shrestha B, Spatafora JW (2007) Phylogenetic classification of Cordyceps and the clavicipitaceous fungi. Stud Mycol 57:5-59

Sunitha VH, Ramesha A, Savitha J, Srinivas C (2012) Amylase production by endophytic fungi Cylindrocephalum sp isolated from medicinal plant Alpinia calcarata (haw) Roscoe. Braz J Microbiol 43:1213-1221

Surup F, Kuhnert E, Lehmann E, Heitkämper S et al (2014) Sporothriolide derivatives as chemotaxonomic markers for Hypoxylon monticulosum. Mycol Int J Fungal Biol 5:110-119

Surup F, Narmani A, Wendt L, Pfütze S, Kretz R, Becker K, Menbrivès C, Giosa A, Elliott M, Petit C, Rohde M, Stadler M (2018) Identification of fungal fossils and novel azaphilone pigments in ancient carbonised specimens of Hypoxylon fragiforme from forest soils of Châtillon-sur-Seine (Burgundy). Fungal Divers 92:345-356

Suryanarayan S (2003) Current industrial practice in solid state fermentations for secondary metabolite production: the Biocon India experience. Biochem Eng J 13:189-195

Suryanarayanan TS, Rajulu G, Vidal S (2016) Biological control through fungal endophytes: gaps in knowledge hindering success. Curr Biotechnol 5:1-13

Suryanarayanan TS, Gopalan V, Shaanker RU, Sengupta A, Ravikanth G (2017) Translating endophyte research to applications: prospects and challenges. In: de Azevedo JL, Quecine MC (eds) Diversity and benefits of microorganisms from the tropics. Springer, Cham, pp 343-365

Sutton BC (1973) Hyphomycetes from Manitoba and Saskatchewan, Canada. Mycol Pap 132:1-143

Suwannarach N, Kumla J, Bussaban B, Lumyong S (2012) Biocontrol of Rhizoctonia solani AG-2, the causal agent of damping off by Muscodor cinnamomi CMU-Cib 461. World J Microbiol Biotechnol 28:3171-3177

Suwannarach N, Kumla J, Bussaban B, Hyde KD, Matsui K, Lumyong S (2013a) Molecular and morphological evidence support four new species in the genus Muscodor from northern Thailand. Ann Microbiol 63:1341-1351

Suwannarach N, Kumla J, Bussaban B, Nuangmek W et al (2013b) Mycofumigation with an endophytic fungus, Nodulisporium spp. CMU-UPE34 for control of citrus fruit decay. Crop Protect 45:63-70

Suwannarach N, Bussaban B, Nuangmek W, Pithakpol W et al (2015a) Evaluation of Muscodor suthepensis CMU-Cib462 as a postharvest biofumigant for tangerine fruit rot caused by Penicillium digitatum. J Sci Food Agric 96:339-345

Suwannarach N, Kumla J, Matsui K, Lumyong S (2015b) Characterization and efficacy of Muscodor cinnamomi in promoting plant growth and controlling Rhizoctonia root rot in tomatoes. Biol Control 90:25-33

Suwannarach N, Kaewyana C, Yodmeeklin A, Kumla J et al (2017) Evaluation of Muscodor cinnamomi as an egg biofumigant for the reduction of microorganisms on eggshell surfaces and its effect on egg quality. Int J Food Microbiol 244:52-61

Swatloski RP, Spear SK, Holbrey JD, Rogers RD (2002) Dissolution of cellulose with ionic liquids. J Am Chem Soc 124:4974-4975

Synytsya A, Míčková K, Synytsya A, Jablonský I et al (2009) Glucans from fruit bodies of cultivated mushrooms Pleurotus ostreatus and Pleurotus eryngii: structure and potential prebiotic activity. Carbohydr Polym 76:548-556

Szabo M (2014) Potential of Trichoderma species and nematodetrapping fungi to control plant-parasitic nematodes: in vitro confrontation and gene expression assays using Caenorhabditis elegans model system

Szajewska H, Konarska Z, Kołodziej M (2016) Probiotic bacterial and fungal strains: claims with evidence. J Dig Dis 34:251-259
Szczodrak J, Fiedurek J (1996) Technology for conversion of lignocellulosic biomass to ethanol. Biomass Bioenerg 10:367375

Sánchez C (2004) Modern aspects of mushroom culture technology. Appl Microbiol Biotechnol 64:756-762

Süssmuth R, Müller J, von Döhren H, Molnár I (2011) Fungal cyclooligomer depsipeptides: from classical biochemistry to combinatorial biosynthesis. Nat Prod Rep 28:99-124

Taira J, Toyoshima R, Ameku N, Iguchi A, Yasutomo TY (2018) Vanillin production by biotransformation of phenolic compounds in fungus, Aspergillus luchuensis. AMB Expr 8:40-48

Takaku T, Kimura Y, Okuda H (2001) Isolation of an antitumor compound from Agaricus blazei Murrill and mechanism of action. J Nutr 5:1409-1413

Tamuli P, Boruah P, Nath SC, Leclercq P (2005) Essential oil of eaglewood tree: a product of pathogenesis. J Essent Oil Res 17:601-604

Tan XM, Wang CL, Chen XM, Zhou YQ et al (2014) In vitro seed germination and seedling growth of an endangered epiphytic orchid, Dendrobium officinale, endemic to China using mycorrhizal fungi (Tulasnella sp.). Sci Hortic 165:62-68

Tan S, Yang B, Liu J, Xun T et al (2017) Penicillixanthone A, a marine-derived dual-coreceptor antagonist as anti-HIV-1 agent. Nat Prod Res 19:1-5

Tan CS, Isa NM, Zainal Z (2019) Agarwood induction: current developments and future perspectives. Front Plant Sci 10:122

Tang H, Huang W, Ma J, Liu L (2018) SWOT analysis and revelation in traditional Chinese medicine internationalization. Chin Med 13:1-9

Taofiq O, Martins A, Barreiro MF, Ferreira IC (2016) Antiinflammatory potential of mushroom extracts and isolated metabolites. Trends Food Sci Technol 50:193-210

Tapingkae T (2005) Mushroom growers' handbook 2, shiitake cultivation, Part II: Mushroom for better life, regional studies: mushroom growing in Lao PDR. MushWorld, Seoul, pp 244 259

Taylor AFS, Alexander I (2005) The ectomycorrhizal symbiosis: life in the real world. Mycologist 9:102-112

Tedersoo L, May TW, Smith ME (2010) Ectomycorrhizal lifestyle in fungi: global diversity, distribution, and evolution of phylogenetic lineages. Mycorrhiza 20:217-263

Teng PS (2008) Bioscience entrepreneurship in Asia: creating value with biology. World Scientific, Hackensack

Teng BS, Wang CD, Yang HJ, Wu JS et al (2011) A protein tyrosine phosphatase 1B activity inhibitor from the fruiting bodies of Ganoderma lucidum (Fr.) Karst and its hypoglycemic potency on streptozotocin-induced type 2 diabetic mice. J Agric Food Chem 59:6492-6500

Teng BS, Wang CD, Zhang D, Wu JS et al (2012) Hypoglycemic effect and mechanism of a proteoglycan from Ganoderma lucidum on streptozotocin-induced type 2 diabetic rats. Eur Rev Med Pharmacol Sci 16:166-175

Tennakoon MMD, Gunatilleke IAUN, Hafeel KM, Seneviratne G (2005) Ectomycorrhizal colonization and seedling growth of Shorea (Dipterocarpaceae) species in simulated shade environments of a Sri Lankan rain forest. For Ecol Manag 208:399-405

Teste FP, Simard SW, Durall DM (2009) Role of mycorrhizal networks and tree proximity in ectomycorrhizal colonization of planted seedlings. Fungal Ecol 2:21-30

Thakur M, Azmi W (2013) Extraction and purification of $\beta$-carotene from filamentous fungus Mucor azygosporus. Ann Phytomed 2 (2):79-84

Thakur MS, Karanth NG, Nand K (1990) Production of fungal rennet by Mucor miehei using solid state fermentation. Appl Microbiol Biot 32:409-413 
Thamer S, Schädler M, Bonte D, Ballhorn DJ (2011) Dual benefit from a belowground symbiosis: nitrogen fixing rhizobia promote growth and defense against a specialist herbivore in a cyanogenic plant. Plant Soil 34:1209-1219

Thawthong A, Karunarathna SC, Thongklang N, Chukeatirote E et al (2014) Discovering and domesticating wild tropical cultivatable mushrooms. Chiang Mai J Sci 41:1-34

Thawthong A, Hapuarachchi KK, Wen TC, Raspé O et al (2017) Ganoderma sichuanense (Ganodermataceae, Polyporales) new to Thailand. MycoKeys 22:27-43

Thomas AT, Shipman FM (2016) Distilled spirit: in Britannica online encyclopedia (access date, January 2019). Encyclopaedia Britannica, Inc., Chicago

Thongbai B, Rapior S, Hyde KD, Wittstein K, Stadler M (2015) Hericium erinaceus, an amazing medicinal mushroom. Mycol Prog 14:1-23

Thongklang N, Luangharn T (2016) Testing agricultural wastes for the production of Pleurotus ostreatus. Mycosphere 7:766-772

Thongklang N, Hoang E, Estrada AER, Sysouphanthong $\mathrm{P}$ et al (2014a) Evidence for amphithallism and broad geographical hybridization potential among Agaricus subrufescens isolates from Brazil, France, and Thailand. Fungal Biol 118:1013-1023

Thongklang N, Sysouphanthong P, Callac P, Hyde KD (2014b) First cultivation of Agaricus flocculosipes and a novel Thai strain of A. subrufescens. Mycosphere 5:814-820

Thongklang N, Chen J, Bandara AR, Hyde KD et al (2016) Studies on Agaricus subtilipes, a new cultivatable species from Thailand, incidentally reveal the presence of Agaricus subrufescens in Africa. Mycoscience 57(4):239-250

Thongklang N, Thongbai B, Chamyuang S, Callac P et al (2017) Blazeispirol A, a chemotaxonomic marker from mycelia of the medicinal mushroom Agaricus subrufescens. Chiang Mai J Sci 44:298-308

Thongbai B, Wittstein K, Richter C, Miller SL et al (2017) Successful cultivation of a valuable wild strain of Lepista sordida from Thailand. Mycol Prog 16:211-223

Tian SP, Fan Q, Xu Y, Qin GZ, Liu HB (2002) Effect of biocontrol antagonists applied in combination with calcium on the control of postharvest diseases in different fruit. Bulletin-OILB/SROP 25:193-196

Tian XF, Fang Z, Guo F (2012) Impact and prospective of fungal pretreatment of lignocellulosic biomass for enzymatic hydrolysis. Biofuels Bioprod Bioref 6:335-350

Tian JJ, Gao XX, Zhang WM, Wang L, Qu LH (2013) Molecular identification of endophytic fungi from Aquilaria sinensis and artificial agarwood induced by pinholes-infusion technique. Afr J Biotechnol 12:3115-3131

Tian Y, Lin X, Wang Z, Zhou X et al (2016) Asteltoxins with antiviral activities from the marine sponge-derived fungus Aspergillus sp. SCSIO XWS02F40. Molecules 21:34/1-34/10

Tibpromma S, Hyde KD, Jeewon R, Maharachchikumbura SSN et al (2017) Fungal diversity notes 491-602: taxonomic and phylogenetic contributions to fungal taxa. Fungal Divers 83:1-261

Tibpromma S, Hyde KD, McKenzie EHC, Bhat DJ et al (2018) Fungal diversity notes 840-928: micro-fungi associated with Pandanaceae. Fungal Divers 92:1-160

Tigini V, Prigione V, Toro SD, Fava F, Giovanna CV (2009) Isolation and characterisation of polychlorinated biphenyl (PCB) degrading fungi from a historically contaminated soil. Microb Cell Factories 8:1-14

Till O (1962) Cultivation of mushrooms on sterile substrate and reutilization of spent compost. Mush Sci 5:127-133

Tokai T, Koshino H, Takahashi-Ando N, Sato M et al (2007) Fusarium Tri4 encodes a key multifunctional cytochrome P450 monooxygenase for four consecutive oxygenation steps in trichothecene biosynthesis. Biochem Biophys Res Commun 353:412-417

Tokiwa Y, Calabia BP, Ugwu CU, Aiba S (2009) Biodegradability of plastics. Int J Mol Sci 10:3722-3742

Tomsheck AR, Strobel GA, Booth E, Geary B et al (2010) Hypoxylon sp., an endophyte of Persea indica, producing 1,8-cineole and other bioactive volatiles with fuel potential. Microb Ecol 60:903-914

Tomšovský M, Homolka L (2004) Mating tests among geographically separated collections of the Trametes versicolor (Fr.) Pilat (Basidiomycetes, Polyporales) group. Nova Hedwigia 79:425431

Tong CC, Rajendra K (1992) Effect of carbon and nitrogen sources on the growth and production of cellulase enzymes of a newly isolated Aspergillus sp. Pertanika 15:45-50

Topka S, Khalil S, Stanchina E, Vijai J, Offit K (2018) Preclinical evaluation of enhanced irofulven antitumor activity in an ERCC3 mutant background by in vitro and in vivo tumor models. AACR 78:3258

Torres DPM, Goncalves MPF, Teixeir JA, Rodrigues LR (2010) Galacto-oligosaccharides: production properties applications and significance as prebiotics. Compr Rev Food Sci Food Saf 9:438 454

Torres FAE, Zaccarim BR, Novaes LCL, Jozala AF (2016) Natural colorants from filamentous fungi. Appl Microbiol Biotechnol 100:2511-2521

Traquair JA, Meloche RB, Jarvis WR, Baker KW (1984) Hyperparasitism of Puccinia violae by Cladosporium uredinicola. Can $\mathrm{J}$ Bot 62:181-184

Trdá L, Barešová M, Šašek V, Nováková M et al (2017) Cytokinin metabolism of pathogenic fungus Leptosphaeria maculans involves isopentenyltransferase, adenosine kinase and cytokinin oxidase/dehydrogenase. Front Microbiol 8:1374

Trinci AP (1994) Evolution of the Quorn ${ }^{\circledR}$ myco-protein fungus, Fusarium graminearum A3/5. Microbiology 140:2181-2188

Trivedi DK, Bhatt H, Pal RK, Tuteja R et al (2013) Structure of RNAinteracting cyclophilin A-like protein from Piriformospora indica that provides salinity-stress tolerance in plants. Sci Rep 3:3001

Tsuneda A, Hiratsuka Y (1979) Mode of parasitism of a mycoparasite, Cladosporium gallicola, on western gall rust, Endocronartium harknessii. Can J Plant Pathol 1:31-36

Turkekul I, Elmastas M, Tuzen M (2004) Determination of iron, copper, manganese, zinc, lead, and cadmium in mushroom samples from Tokat, Turkey. Food Sci 84:389-392

Ul-Haq I, Idrees S, Rajoka MI (2002) Production of lipases by Rhizopus oligosporus by solid-state fermentation. Process Biochem 37:637-641

Ülker S, Özel A, Çolak A, Alpay Karaoğlu S (2011) Isolation production and characterization of an extracellular lipase from Trichoderma harzianum isolated from soil. Turk J Biol 35:543550

Ullrich R, Huong LM, Dung NL, Hofrichter M (2005) Laccase from the medicinal mushroom Agaricus blazei: production purification and characterization. Appl Microbiol Biotechnol 67:357363

Upadhyay RK (2018) Plant pigments as dietary anticancer agents. Int J Green Pharm 12:93-107

Valentín L, Oesch-Kuisma H, Steffen KT, Kähkönen MA et al (2013) Mycoremediation of wood and soil from an old sawmill area contaminated for decades. J Hazard Mater 260:668-675

Valladao ABG, Sartore PE, Freire DMG, Cammarota MC (2009) Evaluation of different pre-hydrolysis times and enzyme pool concentrations on the biodegradability of poultry slaughterhouse wastewater with a high fat content. Water Sci Technol 60 (1):243-249 
Valladao ABG, Torres AG, Freire DM, Cammarota MC (2011) Profiles of fatty acids and triacylglycerols and their influence on the anaerobic biodegradability of effluents from poultry slaughterhouse. Bioresour Technol 102:7043-7050

Valverde ME, Hernández-Pérez T, Paredes-López O (2015) Edible mushrooms: improving human health and promoting quality life. Int J Microbiol 2015:14

van Lenteren JC (2012) The state of commercial augmentative biological control: plenty of natural enemies, but a frustrating lack of uptake. Biol Control 57:1-20

Van Oevelen D, Spaepen M, Timmermans P, Verachtert H (1977) Microbiological aspects of spontaneous wort fermentation in production of lambic and gueuze. J Inst Brewing 83:356-360

Van Zwieten L, Kimber S, Morris S, Chan KY, Downie A (2010) Effects of biochar from slow pyrolysis of papermill waste on agronomic performance and soil fertility. Plant Soil 327:235246

Vandamme EJ (2003) Bioflavors and fragrances via fungi and their enzymes. Fungal Divers 13:153-166

Varma A, Verma S, Sudha SN et al (1999) Piriformospora indica, a cultivable plant-growth-promoting root endophyte. Appl Environ Microbiol 65:2741-2744

Varma A, Bakshi M, Lou B, Hartmann A, Oelmueller R (2012) Piriformospora indica: a novel plant growth-promoting mycorrhizal fungus. Agric Res 1:117-131

Varriale S, Houbraken J, Granchi Z, Pepe O et al (2018) Talaromyces borbonicus, sp. nov., a novel fungus from biodegraded Arundo donax with potential abilities in lignocellulose conversion. Mycologia 110:316-324

Vassilev N, Vassileva M, Lopez A, Martos V et al (2015) Unexploited potential of some biotechnological techniques for biofertilizer production and formulation. Appl Microbiol Biotechnol 99:4983-4996

Velišek J, Cejpek K (2011) Pigments of higher fungi-a review. Czech J Food Sci 29:87-102

Velmala SM, Vuorinen I, Uimari A, Piri T, Pennanen T (2018) Ectomycorrhizal fungi increase the vitality of Norway spruce seedlings under the pressure of Heterobasidion root rot in vitro but may increase susceptibility to foliar necrotrophs. Fungal Biol 122:101-109

Vendruscolo F, Schmidell W, de Oliveira D, Ninow JL (2017) Kinetic of orange pigment production from Monascus ruber on submerged fermentation. Bioprocess Biosyst Eng 40:115-121

Verachtert H, Dawoud E (1990) Yeast in mixed cultures. Louvain Brewing Lett 3:15-40

Verma S, Varma A, Rexer KH, Hassel A et al (1998) Piriformospora indica, gen. et sp. nov., a new root-colonizing fungus. Mycologia 90:896-903

Veselá M, Friedrich J (2009) Amino acid and soluble protein cocktail from waste keratin hydrolysed by a fungal keratinase of Paecilomyces marquandii. Biotechnol Bioproc Eng 14:84-90

Vessey JK (2003) Plant growth promoting rhizobacteria as biofertilizers. Plant Soil 255:571-586

Vesth TC, Brandl J, Andersen MR (2016) FunGeneClusterS: predicting fungal gene clusters from genome and transcriptome data. Synth Syst Biotechnol 1:122-129

Vetchinkina EP, Nikitina VE, Tsivileva OM, Garibova LV (2008) Activity of Lentinus edodes intracellular lectins at various developmental stages of the fungus. Appl Biochem Micro 44:66-72

Victor OO, Olatomiwa OA (2013) Micro and macronutrient properties of Pleurotus ostreatus (Jacq: Fries) cultivated on different wood substrates. Jordan J Biol Sci 6:223-226

Viecelli CA, Stangarlin JR, Kuhn OJ, Schwan-Estrada KRF (2009) Induction of resistance in beans against Pseudocercospora griseola by culture filtrates of Pycnoporus sanguineus. Trop Plant Pathol 34:87-96

Vieira FR, de Andrade MCN (2016) Optimization of substrate preparation for oyster mushroom (Pleurotus ostreatus) cultivation by studying different raw materials and substrate preparation conditions (composting: phases I and II). World J Microbiol Biotechnol 32(11):190

Vieira GAL, Magrini MJ, Bonugli-Santos RC, Rodrigues MVN, Settea LD (2018) Polycyclic aromatic hydrocarbons degradation by marine-derived basidiomycetes: optimization of the degradation process. Braz J Microbiol 49:749-756

Vinale F, Sivasithamparam K, Ghisalberti EL, Marra R et al (2008) Trichoderma-plant-pathogen interactions. Soil Biol Biochem 40:1-10

Von Nussbaum F, Spiteller P, Ruth M, Steglich W et al (1998) An iron (III)-catechol complex as a mushroom pigment. Angew Chem Int Ed 37:3292-3295

Voříšková J, Baldrian P (2013) Fungal community on decomposing leaf litter undergoes rapid successional changes. ISME J 7:477486

Vysotskaya MR, Maslova GV, Petrova VA, Nud'ga LA (2009) Electrochemical recovery of chitin-glucan complex from Pleurotus ostreatus basidial fungus and properties of the product. Russ J Appl Chem 82:1390-1395

WHO (2004a) Global strategy on diet, physical activity and health. Food Nutr Bull 25:292-302

WHO (2004b) Cancer: global health observatory (GHO) data. WHO, Geneva

WHO (2015) World report on ageing and health. World Health Organization, Geneva

WHO Report (2017) The world is running out of antibiotics, WHO report confirms. https://www.who.int/news-room/detail/20-092017-the-world-is-running-out-of-antibiotics-who-reportconfirms

Wada AC (2003) Control of sugarcane smut disease in Nigeria with fungicides. Crop Prot 22:45-49

Wadsworth CA, Li J-PO, Thillainayagam AV (2011) Symptoms and signs of lower gastrointestinal disease. Medicine 39:72-78

Wahlström RM, Suurnäkki A (2015) Enzymatic hydrolysis of lignocellulosic polysaccharides in the presence of ionic liquids. Green Chem 17:694-714

Waksman SA (1947) What is an antibiotic or an antibiotic substance? Mycologia 39:565-569

Walia US, Kumar N, Brar HS, Brar LS (1999) Effect of straw burning and depth of ash incorporation on the efficacy of isoproturon against Phalaris minor Retz. in wheat. Indian J Weed Sci 31:225-228

Walker HL, Connick WJ Jr (1983) Sodium alginate for production and formulation of mycoherbicides. Weed Sci 31:333-338

Walser J, Heinstein PF (1973) Mode of action of illudin S. Antimicrob Agents Chemother 3:357-363

Wan JMF (2013) Chapter 27 - Polysaccaride Krestin (PSK) and Polysaccharopeptide PSP. In: Kastin AJ (ed) Handbook of Biologically Active Peptides, 2nd edn. Academic Press, New York, pp 180-184

Wanasinghe DN, Phukhamsakda C, Hyde KD, Jeewon R et al (2018) Fungal diversity notes 709-839: taxonomic and phylogenetic contributions to fungal taxa with an emphasis on fungi on Rosaceae. Fungal Divers 89:1-236

Wang HT, Hsu JT (2006) Usage of enzyme substrate to protect the activities of cellulase protease and $\alpha$-amylase in simulations of monogastric animal and avian sequential total tract digestion. Asian-Aust J Anim Sci 19:1164-1173

Wang Q, Xu L (2012) Beauvericin, a bioactive compound produced by fungi: a short review. Molecules 17:2367-2377 
Wang DM, Zhang XQ, Yao YJ (2005a) Type studies of some Ganoderma species from China. Mycotaxon 93:61-70

Wang JC, Hu SH, Wang JT, Chen KS, Chia YC (2005b) Hypoglycemic effect of extract of Hericium erinaceus. J Sci Food Agric 85:641-646

Wang H, Liu L, Guo YX, Dong YS et al (2007) Biotransformation of piceid in Polygonum cuspidatum to resveratrol by Aspergillus oryzae. Appl Microbiol Biotechnol 75:763-768

Wang XG, Wan XC, Hu SX (2008) Study on the increase mechanism of the caffeine content during the fermentation of tea with microorganisms. Food Chem 107:1086-1091

Wang C, Sun H, Li Y, Zhang Q (2009) Enzyme activities during degradation of polycyclic aromatic hydrocarbons by white rot fungus Phanerochaete chrysosporium in soils. Chemosphere 77:733-738

Wang BS, Lee CP, Chen ZT, Yu HM, Duh PD (2012) Comparison of the hepatoprotective activity between cultured Cordyceps militaris and natural Cordyceps sinensis. J Funct Food 4:489-495

Wang Q, Luo W, Gu QY, Feng LR et al (2013a) Enhanced lycopene content in Blakeslea trispora by effective mutation-screening method. Appl Biochem Biotechnol 171:1692-1700

Wang YZ, Zhang J, Zhao YL, Li T et al (2013b) Mycology, cultivation, traditional uses, phytochemistry and pharmacology of Wolfiporia cocos (Schwein.) Ryvarden et Gilb.: a review. J Ethnopharmacol 147:265-276

Wang J, Wei X, Qin X, Tian X et al (2016) Antiviral merosesquiterpenoids produced by the antarctic fungus Aspergillus ochraceopetaliformis SCSIO 05702. J Nat Prod 79:59-65

Wang L, Suo S, Li J, Hu Y et al (2017) An investigation into traditional Chinese medicine hospitals in China: development trend and medical service innovation. Int J Health Policy Manag 6:19-25

Wang PM, Liu XB, Dai YC, Horak E et al (2018a) Phylogeny and species delimitation of Flammulina: taxonomic status of winter mushroom in East Asia and a new European species identified using an integrated approach. Mycol Progr 17:1013-1030

Wang T, Mohr KI, Stadler M, Dickschat J (2018b) Volatiles from the tropical ascomycete Daldinia clavata (Hypoxylaceae, Xylariales). Beilstein J Org Chem 14:135-147

Wang X, Wang C, Sui J, Liu Z et al (2018c) Isolation and characterization of phosphofungi, and screening of their plant growth-promoting activities. AMB Expr 8:1-12

Waqas M, Khan AL, Kamran M, Hamayun M et al (2012) Endophytic fungi produce gibberellins and indoleacetic acid and promotes host-plant growth during stress. Molecules 17:10754-10773

Warnock DD, Lehmann J, Kuyper TW, Rillig MC (2007) Mycorrhizal responses to biochar in soil-concepts and mechanisms. Plant Soil 300:9-20. https://doi.org/10.1007/s11104-007-9391-5

Weber G, Chen H, Hinsch E, Freitas S, Robinson S (2014) Pigments extracted from the wood-staining fungi Chlorociboria aeruginosa, Scytalidium cuboideum, and $S$. ganodermophthorum show potential for use as textile dyes. Color Technol 130:445-452

Weedon JT, Aerts R, Kowalchuk GA, Van Bodegom PM (2011) Enzymology under global change: organic nitrogen turnover in alpine and sub-Arctic soils. Biochem Soc Trans 39:309-314

Wei PL, Xu ZN, Cen PL (2007) Lovastatin production by Aspergillus terreus in solid-state fermentation. J Zhejiang Univ Sci 8:15211526

Wei W, Shu S, Zhu W, Xiong Y, Peng F (2016) The Kinome of edible and medicinal fungus Wolfiporia cocos. Front Microbiol 7:1-10

Wei R, Zimmermann W (2017) Microbial enzymes for the recycling of recalcitrant petroleum-based plastics: how far are we? Microb Biotechnol 10:1308-1322

Weiß M, Waller F, Zuccaro A, Selosse MA (2016) Sebacinales-one thousand and one interactions with land plants. New Phytol 211:20-40
Wen X, Jia Y, Li J (2009) Degradation of tetracycline and oxytetracycline by crude lignin peroxidase prepared from Phanerochaete chrysosporium - a white rot fungus. Chemosphere 75:1003-1007

Wen TC, Long FY, Kang C, Wang F, Zeng W (2017) Effects of additives and bioreactors on cordycepin production from Cordyceps militaris in liquid static culture. Mycosphere 8:886898

Wendt L, Sir EB, Kuhnert E, Heitkämper S et al (2018) Resurrection and emendation of the Hypoxylaceae, recognised from a multigene genealogy of the Xylariales. Mycol Prog 17:115-154

Wheelock V (1993) Quorn: case study of a healthy food ingredient. Br Food J 95:40-44

Whipps JM (2004) Prospects and limitations for mycorrhizas in biocontrol of root pathogens. Can J Bot 82:1198-1227

Wi SG, Cho EJ, Lee DS, Lee SJ et al (2015) Lignocellulose conversion for biofuel: a new pretreatment greatly improves downstream biocatalytic hydrolysis of various lignocellulosic materials. Biotechnol Biofuels 8:228

Wiebe MG (2004) Quorn TM myco-protein-overview of a successful fungal product. Mycologist 18:17-20

Wiesinger D, Borel JF (1980) Studies on the mechanism of action of cyclosporin A. Immunobiology 156:454-463

Wijayawardene NN, Hyde KD, Lumbsch HT, Liu JK et al (2018) Outline of ascomycota: 2017. Fungal Divers 88:167-263

Wikee S, Chumnunti P, Kanghae A, Chukeatirote E et al (2017) Lignocellulolytic capability of endophytic Phyllosticta sp. J Bacteriol Mycol 4:1047-1053

Williams K, Khan A, Holland R (1999) Infection of Meloidogyne javanica by Paecilomyces lilacinus. Nematology 1:131-139

Willis KJ (ed) (2018) State of the World's Fungi 2018. Report. Royal Botanic Gardens, Kew, p 92

Wilson CL (1969) Use of plant pathogens in weed control. Annu Rev Phytopathol 7:422-4434

Wilson DB (2009) Cellulases and biofuels. Curr Opin Biotechnol 20:295-299

Wilson AW, Binder M, Hibbett DS (2011) Effects of fruiting body morphology on diversification rates in three independent clades of fungi estimated using binary state speciation and extinction analysis. Evolution 65:1305-1322

Winiski J (2015) US patent application US9914906B2. U.S. Patent and Trademark Office, Washington, DC

Winston RL, Schwarzländer M, Hinz HL et al (eds) (2014) Biological control of weeds: a world catalogue of agents and their target weeds. FHTET-2014-04, 5th edn. USDA Forest Service, Forest Health Technology Enterprise Team, Morgantown, p 838

Wisitrassameewong K, Karunarathna SC, Thongklang N, Zhao R et al (2012a) Agaricus subrufescens: a review. Saudi J Biol Sci 19:131-146

Wisitrassameewong K, Karunarathna SC, Thongklang N, Zhao RL et al (2012b) Agaricus subrufescens: new records to Thailand, Chiang Mai. J Sci 39:281-291

Wittstein K, Rascher M, Rupcic Z, Löwen E, Winter B, Köster RW, Stadler M (2016) Corallocins A-C, nerve growth and brainderived neurotrophic factor inducing metabolites from the mushroom Hericium coralloides. J Nat Prod 79:2264-2269

Wohlgemuth R (2010) Biocatalysis-key to sustainable industrial chemistry. Curr Opin Biotechnol 21:713-724

Wong YY, Moon A, Duffin R, Barateig AB et al (2010) Cordycepin inhibits protein synthesis and cell adhesion through effects on signal transduction. J Biol Chem 285:2610-2621

Worapong J, Strobel GA (2009) Biocontrol of root rot of kale by Muscodor albus strain MFC2. Biocontrol 54:301-306

Worapong J, Strobel GA, Ford E, Li JY et al (2001) Muscodor albus anam. gen. et sp. nov., an endophyte from Cinnamomum zeylanicum. Mycotaxon 79:67-79 
Wraight SP, Carruthers RI (1998) Production, delivery, and use of mycoinsecticides for control of insect pests on field crops. Methods Biotechnol 5:233-269

Wu PH, Huang DD, Chang DCN (2011) Mycorrhizal symbiosis enhances Phalaenopsis orchid's growth and resistance to Erwinia chrysanthemi. Afr J Biotechnol 10:10095-10100

Wu XL, Mao XL, Tuli GE, Song B et al (2013) Medicinal fungi of China. Science Press, Beijing, China

Wu X, Chen T, Qin P, Hu F, Yang H (2015a) Fungal secondary metabolite 11'-deoxyverticillin A (C42) decreases HBV-X replication by down-regulating autophagy related gene expression. Junwu Xuebao 34:1187-1195

Wu Z, Chen G, Wang M (2015b) Screening and application of Monascus strains with high yield of extracellular yellow pigments. China patent no. CN201510449543.6

Xiang L, Li Y, Zhu Y, Luo H et al (2014) Transcriptome analysis of the Ophiocordyceps sinensis fruiting body reveals putative genes involved in fruiting body development and cordycepin biosynthesis. Genomics 103:154-159

Xiao P, Mori T, Kamei I, Kiyota H et al (2011) Novel metabolic pathways of organochlorine pesticides dieldrin and aldrin by the white rot fungi of the genus Phlebia. Chemosphere 85:218-224

Xu Q, Li S, Huang H, Wen J (2012) Key technologies for the industrial production of fumaric acid by fermentation. Biotechnol Adv 30:1685-1696

Yamac M, Kanbak G, Zeytinoglu M, Senturk H et al (2010) Pancreas protective effect of button mushroom Agaricus bisporus (J.E. Lange) Imbach (Agaricomycetidae) extract on rats with streptozotocin-induced diabetes. Int J Med Mushrooms 12:379-389

Yamaguchi S (2008) Gibberellin metabolism and its regulation. Ann Rev Plant Biol 59:225-251

Yamamaka K (1997) Production of cultivated edible mushrooms. Food Rev Int 13:327-333

Yamanaka D, Liu Y, Motoi M, Ohno N (2013) Royal sun medicinal mushroom, Agaricus brasiliensis Ka21 (Higher Basidiomycetes), as a functional food in humans. Int $\mathrm{J}$ Med Mushrooms 15:335-343

Yamane T, Tanaka R (2013) Highly accumulative production of 1(+)lactate from glucose by crystallization fermentation with immobilized Rhizopus oryzae. J Biosci Bioeng 115:90-95

Yan JF, Broughton SJ, Yang SL, Gange AC (2015) Do endophytic fungi grow through their hosts systemically? Fungal Ecol 13:5359

Yanagihara M, Sasaki-Takahashi N, Sugahara T, Yamamoto S et al (2005) Leptosins isolated from marine fungus Leptoshaeria species inhibit DNA topoisomerases I and/or II and induce apoptosis by inactivation of Akt/protein kinase B. Cancer Sci J 96:816-824

Yang W, Guo F, Wan Z (2013a) Yield and size of oyster mushroom grown on rice wheat straw basal substrate supplemented with cotton seed hull. Saudi J Biol Sci 20:333-338

Yang Y, Wei F, Zhuo R, Fan F et al (2013b) Enhancing the laccase production and laccase gene expression in the white-rot fungus Trametes velutina 5930 with great potential for biotechnological applications by different metal ions and aromatic compounds. PLoS ONE 8:1-9

Yang J, Yang Y, Wu WM, Zhao J, Jiang L (2014a) Evidence of polyethylene biodegradation caterpillars by bacterial strains from the guts of plastic-eating wax worms. Environ Sci Technol 48:13776-13784

Yang L, Lübeck M, Lübeck PS (2014b) Deletion of glucose oxidase changes the pattern of organic acid production in Aspergillus carbonarius. AMB Express 4:1-9

Yang W, Han D, Wu L, Huang Y et al (2016) Hericium erinaceus synergizing with doxorubicin induced SGC7901 cell apoptosis. Int J Clin Exp Med 9:1447-1457
Yao J, Guo GS, Ren GH, Liu YH (2014) Production, characterization and applications of tannase. J Mol Catal B 101:137-147

Yin X, Li J, Shin HD, Du G et al (2015) Metabolic engineering in the biotechnological production of organic acids in the tricarboxylic acid cycle of microorganisms: advances and prospects. Biotechnol Adv 33:830-841

Ying J, Mao X, Ma Q, Wen H (1987) Icons of medicinal fungi from China. Science, Beijing

Yoder JA, Zettler LW, Stewart SL (2000) Water requirements of terrestrial and epiphytic orchid seeds and seedlings, and evidence for water uptake by means of mycotrophy. Plant Sci 156:145150

Yongsmith B, Thongpradis P, Klinsupa W, Chantrapornchai W, Haruthaithanasan V (2013) Fermentation and quality of yellow pigments from golden brown rice solid culture by a selected Monascus mutant. Appl Microbiol Biotechnol 97:8895-8902

Yoon YH, Choi SH, Cho HJ, Moon SW, Kim JY, Lee S (2011) Reversible pancytopenia following the consumption of decoction of Ganoderma neojaponicum Imazeki. Clin Toxicol 49(2):115117

Yoshida K, Burbano HA, Krause J, Thines M et al (2014) Mining herbaria for plant pathogen genomes: back to the future. PLoS Pathog 10:1-6

Young D, Dollhofer V, Callaghan TM, Reitberger S (2018) Isolation, identification and characterization of lignocellulolytic aerobic and anaerobic fungi in one-and two-phase biogas plants. Bioresour Technol 268:470-479

Yu L, Wang Y, Yuan Y, Tang J, Zhou S (2016) Biochar as electron acceptor for microbial extracellular respiration. Geomicrobiol $\mathrm{J}$ 33:530-536

Yuan Z, Druzhinina IS, Labbé J, Redman R et al (2016) Specialized microbiome of a halophyte and its role in helping non-host plants to withstand salinity. Sci Rep 6:1-13

Yue Q, Chen L, Zhang X, Li K et al (2015) Evolution of chemical diversity in the echinocandin lipopeptide antifungal metabolites. Eukaryotic Cell 14(7):76

Yun HG, Kim DJ, Gwak WS, Shin TY, Woo SD (2017) Entomopathogenic fungi as dual control agents against both the pest Myzus persicae and phytopathogen Botrytis cinerea. Mycobiology 45(192): 198

Yun P, Xu L, Wang SS, Shabala L et al (2018) Piriformospora indica improves salinity stress tolerance in Zea mays L. plants by regulating $\mathrm{Na}+$ and $\mathrm{K}+$ loading in root and allocating $\mathrm{K}+$ in shoot. Plant Growth Regul 86:323-331

Zafar U, Houlden A, Robson GD (2013) Fungal communities associated with the biodegradation of polyester polyurethane buried under compost at different temperatures. Appl Environ Microbiol 79:7313-7324

Zafar U, Nzeram P, Langarica-Fuentes A, Houlden A et al (2014) Biodegradation of polyester polyurethane during commercial composting and analysis of associated fungal communities. Bioresour Technol 158:374-377

Zafra G, Cortés-Espinosa DV (2015) Biodegradation of polycyclic aromatic hydrocarbons by Trichoderma species: a mini review. Environ Sci Pollut Res 22:19426

Zain MMM, Rosli BM, Kamaruzaman S et al (2013) Effects of selected herbicides on soil microbial populations in oil palm plantation of Malaysia. A microcosm experiment. Afr J Microbiol Res 7:367-374

Zaki A, Siddiqui MI (1996) Biological control of plant parasitic nematodes by fungi. Bioresour Technol 58:229-239

Zelmer CD, Cuthbertson L, Currah RS (1996) Fungi associated with terrestrial orchid mycorrhizas, seeds and protocorms. Mycoscience 37:439-448

Zervakis GI, Koutrotsios G, Katsaris P (2013) Composted versus raw olive mill waste as substrates for the production of medicinal 
mushrooms: an assessment of selected cultivation and quality parameters. Biomed Res Int 2013:1-13

Zhang SH, Wei Y (2017) Applications of haloalkaliphilic fungi in mycoremediation of saline-alkali soil. In: Prasad R (ed) Mycoremediation and environmental sustainability. Fungal biology. Springer, Cham

Zhang M, Cheung PCK, Zhang L (2001) Evaluation of mushroom dietary fiber (nonstarch polysaccharides) from sclerotia of Pleurotus tuber-regium (Fries) Singer as a potential antitumor agent. J Agric Food Chem 49:5059-5062

Zhang R, Li X, Fadel JG (2002) Oyster mushroom cultivation with rice and wheat straw. Bioresour Technol 82:277-284

Zhang ZY, Jin B, Kelly JM (2007) Production of lactic acid from renewable materials by Rhizopus fungi. Biochem Eng J 35:251263

Zhang J, Zhu Z, Wang X, Wang N et al (2010a) Biodetoxification of toxins generated from lignocellulose pretreatment using a newly isolated fungus, Amorphotheca resinae $\mathrm{ZN1}$, and the consequent ethanol fermentation. Biotechnol Biofuels 3:1-15

Zhang X, Shi Y, Wang X, Zhang W, Lou K (2010b) Isolation, identification and insecticidal activity of endophyte from Achnatherum inebrins. Acta Microbiol Sin 50:530-536

Zhang J, Yu Y, Zhang Z, Ding Y et al (2011a) Effect of polysaccharide from cultured Cordyceps sinensis on immune function and anti-oxidation activity of mice exposed to 60Co. Int Immunopharmacol 11:2251-2257

Zhang T, Datta S, Eichler J, Ivanova N et al (2011b) Identification of a haloalkaliphilic and thermostable cellulase with improved ionic liquid tolerance. Green Chem 13:2083-2090

Zhang RH, Duan ZQ, Li ZG (2012) Use of spent mushroom substrate as growing media for tomato and cucumber seedlings. Pedosphere 22:333-342

Zhang Y, Geng W, Shen Y, Wang Y, Dai YC (2014a) Edible mushroom cultivation for food security and rural development in China: bio-innovation, technological dissemination and marketing. Sustainability 6:2961-2973

Zhang Z, Han X, Wei J, Xue J (2014b) Compositions and antifungal activities of essential oils from agarwood of Aquilaria sinensis (Lour.) Gilg induced by Lasiodiplodia theobromae (Pat.) Griffon. and Maubl. J Braz Chem Soc 25:20-26

Zhang JX, Chen Q, Huang CY, Gao W, Qu JB (2015) History, current situation and trend of edible mushroom industry development. Mycosystema 34:524-540

Zhang S, Gan Y, Xu B (2016a) Application of plant-growthpromoting fungi Trichoderma longibrachiatum T6 enhances tolerance of wheat to salt stress through improvement of antioxidative defense system and gene expression. Front Plant Sci 7:1-11

Zhang S, Huang R, Li F, Wei H et al (2016b) Antiviral anthraquinones and azaphilones produced by an endophytic fungus Nigrospora sp. from Aconitum carmichaeli. Fitoterapia 112:85-89

Zhang Y, Skaar I, Sulyok M et al (2016c) The microbiome and metabolites in fermented Pu-erh tea as revealed by highthroughput sequencing and quantitative multiplex metabolite analysis. PLoS ONE 11:1-18

Zhang Y, Navarro E, Cánovas-Márquez JT, Almagro L, Chen H, Chen YQ, Zhang H, Torres-Martínez S, Chen W, Garre V (2016d) A new regulatory mechanism controlling carotenogenesis in the fungus Mucor circinelloides as a target to generate $\beta$ carotene over-producing strains by genetic engineering. Microb Cell Fact 15:99

Zhang CC, Cao CY, Kubo M, Harrada K et al (2017a) Chemical constituents from Hericium erinaceus promote neuronal survival and potentiate neurite outgrowth via the TrkA/Erk1/2 pathway. Int J Mol Sci 18:1-13
Zhang X, Zhou Y, Li Y, Fu X, Wang Q (2017b) Screening and characterization of endophytic Bacillus for biocontrol of grapevine downy mildew. Crop Prot 96:173-179

Zhang L, Shi N, Fan J, Wang F (2018) Arbuscular mycorrhizal fungi stimulate organic phosphate mobilization associated with changing bacterial community structure under field conditions. Environ Microbiol 20:2639-2651

Zhou SL, Liu XW (2011) Study on preparation technology of Termitomyces albuminosus complex seasoning. China Condiment 36:56-58 In Chinese

Zhou W, Mo M (2002) Soil fungistasis on the spore germination of fungi. J Yunnan Univ (Nat Sci) 24:312-315

Zhao LF, Zhou HJ (2005) Study on the main microbes of Yunnan pu' er tea during pile-fermentation process. J Shangqiu Teach Coll 21:129-133

Zhao LF, Xu YJ, Zhou HJ (2006) Research on microbes improve quality and flavor of pu'er tea in solid fermentation. Food Res Dev 27:155-166

Zhao M, Li M, Liu RJ (2010) Effects of arbuscular mycorrhizae on microbial population and enzyme activity in replant soil used for watermelon production. Int J Eng Sci Technol 2:17-22

Zhao RL, Hyde KD, Desjardin DE, Raspé O (2012) Agaricus flocculosipes sp. nov., a new potentially cultivatable species from the palaeotropics. Mycoscience 53:300-311

Zhao ZJ, Hu XC, Liu QJ (2015) Recent advances on the fungi of Puerh ripe tea. Int Food Res J 22:1240-1246

Zhao J, Feng J, Tan Z, Liu J et al (2017a) Stachybotrysins A-G, Phenylspirodrimane Derivatives from the Fungus Stachybotrys chartarum. J Nat Prod 80:1819-1826

Zhao J, Liu J, Shen Y, Tan Z et al (2017b) Stachybotrysams A-E, prenylated isoindolinone derivatives with anti-HIV activity from the fungus Stachybotrys chartarum. Phytochem Lett 20:289-294

Zhou HJ, Li JH, Zhao LF, Han J et al (2004) Study on main microbes on quality formation of Yunnan Puer tea during pile-fermentation process. J Tea Sci 24:212-218

Zhou J, Xie G, Yan X (2011) Encyclopedia of traditional Chinese medicines-molecular structures, pharmacological activities, natural sources and applications. Isolated compounds H-M, vol 3. Springer, London

Zhou XW, Su KQ, Zhang YM (2012) Applied modern biotechnology for cultivation of Ganoderma and development of their products. Appl Microbiol Biotechnol 93:941-963

Zhou H, Li L, Wang W, Che Q et al (2015a) Chrodrimanins I and J from the Antarctic moss-derived fungus Penicillium funiculosum GWT2-24. J Nat Prod 78:1442-1445

Zhou S, Raouche S, Grisel S, Navarro D et al (2015b) Solid-state fermentation in multi-well plates to assess pretreatment efficiency of rot fungi on lignocellulose biomass. Microb Biotechnol 8:940-949

Zhou LS, Tang K, Guo SX (2018) The plant growth-promoting fungus (PGPF) Alternaria sp. A13 markedly enhances Salvia miltiorrhiza root growth and active ingredient accumulation under greenhouse and field conditions. Int J Mol Sci 19:1-14

Zhu J (2018) Through a century: traditional Chinese medicine since 1912. Chin Med 1:1-10

Zhu W, Wei W, Zhang S, Zheng Y et al (2018) The phosphatome of medicinal and edible fungus Wolfiporia cocos. Curr Microbiol 75:124-131

Zied DC, Pardo-Giménez A (2017) Edible and medicinal mushrooms: technology and applications. Wiley, West Sussex

Zied DC, Minhoni MTA, Kopytowski-Filho J, Andrade MCN (2010) Production of Agaricus blazei ss. Heinemann (A. brasiliensis) on different casing layers and environments. World J Microbiol Biotechnol 26:1857-1863

Zied DC, Pardo-Gimenez A, Savoie JM, Pardo-Gonzalez JR, Callac P (2011) "Indoor" method of composting and genetic breeding of 
the strains to improve yield and quality of the almond mushroom Agaricus subrufescens. In: Savoie JM, Foulongne-Oriol M, Largeteau M, Barroso G (eds) Proceedings of the 7th international conference on mushroom biology and mushroom products, Vol 1. Bouleaux, French National Institute of Agronomical Research, pp 424-432

Zied DC, Pardo JE, Tomaz RS, Miasaki CT, Pardo-Giménez A (2017) Mycochemical characterization of Agaricus subrufescens considering their morphological and physiological stage of maturity on the traceability process. Biomed Res Int 7422713:1-10

Zimdahl RL (1993) Fundamentals of weed science. Academic Press, San Diego
Zoecklein B (2002a) Red wine phenolic management. Enology notes \#61. http://www.apps.fst.vt.edu/extension/enology/EN/61.html

Zoecklein B (2002b) Red wine phenolic management, continued. Enology notes \#62. http://www.apps.fst.vt.edu/extension/enol ogy/EN/62.html

Zong K, Huang J, Nara K, Chen Y et al (2015) Innoculation of ectomycorrhizal fungi contributes to the survival of tree seedlings in a copper mine tailing. J For Res 20:493-500

Zugazagoitia J, Guedes C, Ponce S, Ferrer I, Molina-Pinelo S, PazAres L (2016) Current challenges in cancer treatment. Clin Ther 38:1551-1566

\section{Affiliations}

Kevin D. Hyde ${ }^{1,2,3,4,5,9} \cdot$ Jianchu $X^{1,10,21} \cdot$ Sylvie Rapior ${ }^{22} \cdot$ Rajesh Jeewon $^{18}$ - Saisamorn Lumyong ${ }^{9,13}$. Allen Grace T. Niego ${ }^{2,3,20}$ - Pranami D. Abeywickrama ${ }^{2,3,7} \cdot$ Janith V. S. Aluthmuhandiram ${ }^{2,3,7}$. Rashika S. Brahamanage ${ }^{2,3,7}$. Siraprapa Brooks ${ }^{3}$. Amornrat Chaiyasen ${ }^{28} \cdot$ K. W. Thilini Chethana ${ }^{2,3,7}$. Putarak Chomnunti ${ }^{2,3}$. Clara Chepkirui ${ }^{12}$ - Boontiya Chuankid ${ }^{2,3}$ - Nimali I. de Silva ${ }^{1,2,4,13}$. Mingkwan Doilom ${ }^{1,4,13}$. Craig Faulds $^{6}$. Eleni Gentekaki ${ }^{3}$. Venkat Gopalan ${ }^{14}$ - Pattana Kakumyan ${ }^{2,3}$. Dulanjalee Harishchandra ${ }^{2,3,7}$. Hridya Hemachandran ${ }^{24}$. Sinang Hongsanan ${ }^{26,27}$. Anuruddha Karunarathna ${ }^{2,17}$. Samantha C. Karunarathna ${ }^{1}$ - Sehroon Khan ${ }^{10}$ - Jaturong Kumla ${ }^{13,9}$ - Ruvishika S. Jayawardena ${ }^{2,3}$. Jian-Kui Liu' ${ }^{11}$ - Ningguo Liu'2,3 - Thatsanee Luangharn'1,21,22,29 - Allan Patrick G. Macabeo ${ }^{12,23}$. Diana S. Marasinghe ${ }^{2,3} \cdot$ Dan Meeks $^{19}$ - Peter E. Mortimer ${ }^{1,10}$ - Peter Mueller ${ }^{19} \cdot$ Sadia Nadir $^{10,15,21}$. Karaba N. Nataraja ${ }^{16}$ - Sureeporn Nontachaiyapoom ${ }^{3} \cdot$ Meghan O'Brien $^{19} \cdot$ Watsana Penkhrue $^{9,13}$. Chayanard Phukhamsakda ${ }^{2,3}$. Uma Shaanker Ramanan ${ }^{16,25}$. Achala R. Rathnayaka ${ }^{2,3}$ - Resurreccion B. Sadaba ${ }^{29}$. Birthe Sandargo $^{12}$ - Binu C. Samarakoon ${ }^{2,3}$. Danushka S. Tennakoon ${ }^{2,3} \cdot$ Ramamoorthy Siva $^{24}$. Wasan Sriprom $^{9,13} \cdot$ T. S. Suryanarayanan ${ }^{30} \cdot$ Kanaporn Sujarit $^{9,13} \cdot$ Nakarin Suwannarach $^{9,13}$. Thitipone Suwunwong ${ }^{3,8}$ - Benjarong Thongbai ${ }^{12}$ - Naritsada Thongklang ${ }^{2}$ - Deping Wei $^{1,2,3,17}$.

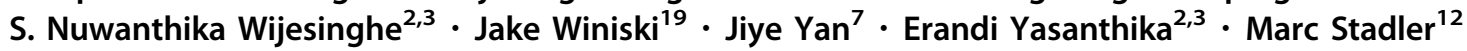

Jianchu Xu j.c.xu@cgiar.org

1 Key Laboratory for Plant Diversity and Biogeography of East Asia, Kunming Institute of Botany, Chinese Academy of Science, Kunming 650201, Yunnan, People's Republic of China

2 Center of Excellence in Fungal Research, Mae Fah Luang University, Chiang Rai 57100, Thailand

3 School of Science, Mae Fah Luang University, Chiang Rai 57100, Thailand

4 World Agroforestry Centre, East and Central Asia, Kunming 650201, Yunnan, People's Republic of China

5 Mushroom Research Foundation, 128 M.3 Ban Pa Deng T. PaPae, A. Mae Taeng, Chiang Mai 50150, Thailand

6 Aix Marseille Université, INRA, UMR1163 BBF, 13288 Marseille Cedex 09, France

7 Beijing Key Laboratory of Environment Friendly Management on Fruit Diseases and Pests in North China, Center of Plant and Environment Protection, Beijing Academy of Agriculture and Forestry Sciences, Beijing 100097, People's Republic of China
8 Center of Chemical Innovation for Sustainability, Mae Fah Luang University, Tasud, Muang, Chiang Rai 57100, Thailand

9 Center of Excellence in Microbial Diversity and Sustainable Utilization, Faculty of Science, Chiang Mai University, Chiang Mai 50200, Thailand

10 Center for Mountain Ecosystem Studies, Kunming Institute of Botany, Chinese Academy of Sciences, Kunming 650201Yunnan, People's Republic of China

11 Institute of Fungal Research, School of Life Science and Technology, University of Electronic Science and Technology of China, Chengdu 611731, People's Republic of China

12 Department Microbial Drugs, Helmholtz Centre for Infection Research, and German Centre for Infection Research (DZIF), partner site Hannover-Braunschweig, Inhoffenstrasse 7, 38124 Brunswick, Germany

13 Department of Biology, Faculty of Science, Chiang Mai University, Chiang Mai 50200, Thailand

14 Department of Chemistry and Biochemistry, The Ohio State University, Columbus, OH 43210, USA

15 Department of Chemistry, University of Science and Technology, Bannu, Khyber Pakhtunkhwa, Pakistan 
16 Department of Crop Physiology, University of Agricultural Sciences, GKVK, Bengaluru, Karnataka 560065, India

17 Department of Entomology and Plant Pathology, Faculty of Agriculture, Chiang Mai University, Chiang Mai 50200, Thailand

18 Department of Health Sciences, Faculty of Science, University of Mauritius, Reduit, Mauritius

19 Ecovative Design, LLC 70 Cohoes Ave, Green Island, NY 12183, USA

20 Iloilo Science and Technology University, La Paz, 5000 Iloilo, Philippines

21 Key Laboratory for Economic Plants and Biotechnology, Kunming Institute of Botany, Chinese Academy of Sciences, Kunming 650201, Yunnan, People's Republic of China

22 Laboratory of Botany, Phytochemistry and Mycology, Faculty of Pharmacy, CEFE CNRS - Université de Montpellier - Université Paul-Valéry Montpellier - EPHE IRD, BP 14491, 15 avenue Charles Flahault, 34093 Montpellier Cedex 5, France

23 Laboratory for Organic Reactivity, Discovery and Synthesis (LORDS), Research Center for the Natural and Applied Sciences, University of Santo Tomas, 1015 Manila, Philippines
24 School of Bio Sciences and Technology, VIT University, Vellore 632 014, India

25 School of Ecology and Conservation, University of Agricultural Sciences, GKVK, Bengaluru, Karnataka 560065, India

26 Shenzhen Key Laboratory of Laser Engineering, College of Optoelectronic Engineering, Shenzhen University, Shenzhen, People's Republic of China

27 Shenzhen Key Laboratory of Microbial Genetic Engineering, College of Life Sciences and Oceanography and Shenzhen University, Shenzhen, People's Republic of China

28 Soil Science Research Group, Agricultural Production Science Research and Development Division, Department of Agriculture, Ministry of Agriculture and Cooperatives, Bangkok 10900, Thailand

29 University of the Philippines Visayas, Miagao, 5023 Iloilo, Philippines

30 Vivekananda Institute of Tropical Mycology, Ramakrishna Mission Vidyapith, Chennai 600004, India 